\title{
Report of Research Activities
}

and Accomplishments of Experimental High Energy Physics Group at Southern Methodist University

T. Coan, J. Dominick, V. Fadeyev, I. Korolkov, M. Lambrecht, G. Li, S. Sanghera, V. Shelkov, T. Skwarnicki, R. Stroynowski, I. Volobouev, G. Wei and P. Zadorozhny

DOE grant DE-FG05-92ER40722 


\section{Personnel}

Faculty

Tom Coan - Assistant Professor

Tomasz Skwarnicki - Assistant Professor

Ryszard Stroynowski - Professor

Postdoctoral Fellows

Jay Dominick - Research Associate Mark Lambrecht - Research Associate

Sukhpal Sanghera - Research Associate

\section{Graduate Students}

Vitalii Fadeyev

Ilya Korolkov

$\mathrm{Ge} \mathrm{Li}$

Vasilii Shelkov

Igor Volobouev

Guoheng Wei

Pavel Zadorozhny

$\underline{\text { Staff }}$

Michael Esaili - Machinist

Rakesh Jain - Computer manager 


\section{DISCLAIMER}

This report was prepared as an account of work sponsored by an agency of the United States Government. Neither the United States Government nor any agency thereof, nor any of their employees, make any warranty, express or implied, or assumes any legal liability or responsibility for the accuracy, completeness, or usefulness of any information, apparatus, product, or process disclosed, or represents that its use would not infringe privately owned rights. Reference herein to any specific commercial product, process, or service by trade name, trademark, manufacturer, or otherwise does not necessarily constitute or imply its endorsement, recommendation, or favoring by the United States Government or any agency thereof. The views and

opinions of authors expressed herein do not necessarily state or reflect those of the United States Government or any agency thereof. 


\section{DISCLAIMER}

Portions of this document may be illegible in electronic image products. Images are produced from the best available original document. 


\section{Content}

\section{Overview}

\section{The CLEO II Program}

2.1 Data reconstruction

2.2 Tau physics group

2.3 Physics Analyses

2.3.1 B physics

Inclusive $b \rightarrow s \gamma$ decays

Semileptonic decays of $B$

Search for semileptonic $B$ decays to charmed baryons

2.3.2 Tau physics

Search for flavor violating decays

Precision measurement of 3-prong branching fractions

Tau Decays to $\nu_{\tau} K_{s}^{0} K \pi^{0}$

2.3.3 QCD studies

Determination of $\alpha_{s}$ from hadronic spectral moments in $\tau$ decays

Testing of flavor structure of QCD with jet production rates

Other QCD tests with the CLEO II data

Phenomenological papers

2.3.4 $\Upsilon$ physics

2.4 Future of the CLEO-II program

3. GEM detector at SSC

4. The CLEO - III Project

4.1 Overview of CESR and CLEO upgrade

Overview of the CLEO III Detector Upgrade.

Overview of the CESR Luminosity Upgrade.

4.2 Particle Identification with CLEO III

4.3 Other CLEO-III projects

Beam pipe cooling tests for CLEO-III

Final focus corrector magnet

Drift chamber electronics

\section{ATLAS at LHC}

6. Velocity of light in magnetic field

7. List of publications $1993-1994$

7.1 Publications in refereed journals

7.2 Conference Talks

7.3 Conference Papers

7.4 Semiars and Colloquia

7.5 Proposals and Technical Notes

7.6 Theses

7.7 Papers Submitted for Publication or in Preparation

8. Current and Pending Support 
9. Facilities and Resources

10. Experimental Computing

11. Budget Explanation

Personnel

Travel

Equipment

12. Appendix 1 Liquid Calorimetry Part of the US ATLAS Proposal

13. Appendix 2 Curricula Vitae of the Faculty 


\section{OVERVIEW}

The High Energy Physics program at SMU started in the fall of 1991. The initial program was based on two experiments. The first one was an involvement in the ongoing CLEO-II experiment at CESR, and the second was a long term commitment to the GEM experiment at the SSC. In addition, the original proposal called for establishment of research, technical, and computing capabilities and for the re-institution of the SMU Ph.D. program.

All of those goals have been accomplished with considerable success except for the SSC cancellation which necessitated change of plans for the future. For the past three years the SMU group has had a significant impact on the CLEO-II experiment by undertaking an off-line data processing responsibility, by providing leadership in tau physics studies and by contributing to many analyses. For example 6 out of 27 CLEO papers submitted to the Glasgow conference in 1994 originated at SMU. Among those prof. T. Skwarnicki has been responsible for the new, important measurement of the $b \rightarrow s \gamma$ rate. In addition, SMU provided leadership for an R\&D program on particle identification detectors for the CLEOIII upgrade addressing the feasibility of silica aerogel for Cerenkov light detection. During the period 1991-1993 the SMU faculty had significant involvement in the GEM detector at the SSC. Stroynowski led the GEM Magnet group and Skwarnicki coordinated physics simulation efforts. The closeout process of the GEM magnet coil R\&D program will continue into 1995.

The future plans for the SMU group are based on the continuation of the CLEO-II program and on involvement in two new experiments: CLEO-III at CESR and ATLAS at the LHC. The possibility of initiating a non-accelerator based research effort is also being considered by the group. For the near term future, SMU plans to emphasize the CLEO-III upgrade, concentrating on construction of a particle identification device, and to continue exploiting the successful data collection at CLEO-II. The CLEO-III choice of the technology for particle identification is not yet clear. The R\&D program is still incomplete. At the time of this writing three technology options are considered for the upgrade: Ring Imaging Cerenkov technique, silica aerogel, and high pressure gas tubes for detection of Cerenkov radiation. The final choice will be made by the CLEO Collaboration in the fall of 1994 and SMU will take part in the construction of the selected device. The CLEO upgrade program is scheduled for a completion in fall of 1997. At that time, the ATLAS construction program will be expanded. For the ATLAS experiment at the LHC, the group has chosen to work on the liquid calorimetry. The US based effort on this subsystem is centered at BNL. The considerable expertise in large detector design gained in the work on GEM is directly applicable to LHC.

An additional new research opportunity may be provided by a possible project to search for axions by measuring the velocity of light in a strong magnetic field, using the SSC magnets. A 3 month "Project definition study" sponsored by DOE will conclude at the end of October. At that time, an additional proposal may be submitted if appropriate.

For the past three years, the group's efforts were dominated by the data analysis and computing aspects of the CLEO-II and GEM related work. In summer of 1994 the group was strengthened by addition of Tom Coan, who joined the SMU faculty as an Assistant 
Professor. Coan has accumulated an extensive hardware experience with the L3 experiment at CERN, with his past contributions to SLD and TEXAS design, and in design and construction of balloon-borne cosmic ray detectors. We expect that Coan will lead the SMU detector construction effort. He will be helped by the new SMU machinist/designer Michael Esaili, who came to us with a 20 years of experience in support of high energy physics groups, most recently at the SSC Laboratory.

The budget proposed here represents the minimum cost of continuing operations of the SMU group. In the past, substantial startup funds came from the SSC program and from the Texas National Research Laboratory Commission. Due to SSC cancellation these resources are no longer available.

We anticipate a need for an additional postdoctoral research associate who will concentrate on the ATLAS related work. We also request an upgrade of our computing capabilities. In view of the continuing improvements of the CESR luminosity and ever increasing CLEO data sample, such improvement is necessary.

Apart from the capital equipment request, the budget is dominated by personnel support and travel expenses. The travel to experiments for collaboration meetings, shifts and analysis meetings is a necessary part of the work. In addition, the importance of CLEO data is reflected in large number of conference presentations assigned to SMU group by the CLEO Collaboration at APS, DPF and many topical and international meetings. Nine such presentations have been assigned so far in 1994. The travel associated with ATLAS experiment has two components: travel to CERN to attend collaboration meetings taking place 6 times a year and travel within US, mainly to BNL, for the work on ATLAS calorimetry.

SMU currently owns a 2 Tesla magnet with $15 \mathrm{~cm}$ diameter pole face and a: $10 \mathrm{~cm}$ pole gap. This magnet will be used to test the responses of various electronic and photodetector components in a magnetic field. We request the funds for a purchase of a power supply for this magnet. 


\section{THE CLEO-II PROGRAM}

The CLEO-II experiment operates at the $e^{+} e^{-}$storage ring CESR with the center of mass energy in vicinity of the $\Upsilon(4 S)$ resonance. The $e^{+} e^{-}$collisions in $10 \mathrm{GeV}$ center of mass energy region are particularly rich in interesting physics phenomena. This is a threshold energy for production of $b \bar{b}$ quark pairs. Below the kinematic threshold for production of $B \bar{B}$ mesons, the narrow $\Upsilon$ resonances are observed. They constitute a positronium-like system bound by strong interactions - a unique quark system due to its non-relativistic nature. The fourth $\Upsilon$ resonance is above the $B \bar{B}$ threshold and always decays to $B$ mesons. This is a laboratory for studies of weak decays of the $b$ quark. Tau pairs are produced by continuum $e^{+} e^{-}$annihilations with about the same cross-section as that of the $\Upsilon(4 \mathrm{~S})$. Tau decays weakly to its neutrino and other leptons or hadrons. In contrast to experiments with hadronic beams, productions of $b$ quark pairs and $\tau$ lepton pairs in $e^{+} e^{-}$collisions is free from other accompanying particles, providing a clean environment for studies of $\tau$ and $b$ properties. The CLEO-II program also makes important contributions to physics of the second generation quark $c$ and to the light quark interactions observed in $\gamma \gamma$ collisions.

The electron-positron storage ring CESR at the Cornell University is one of the most successful accelerator programs in history. CESR has a continuous record of improving luminosity performance, achieving an order of magnitude improvement every five years. With peak luminosity of $2.9 \cdot 10^{32} \mathrm{~cm}^{-2} \mathrm{~s}^{-1}$ the CESR is now the highest luminosity $e^{+} e^{-}$ collider in the world. A significant upgrade of the CESR luminosity is anticipated over next couple of years. The projected luminosity by the year 1997 is $1.5 \cdot 10^{33} \mathrm{~cm}^{-2} \mathrm{~s}^{-1}$.

The CLEO-II detector shown in Fig.1. Photons are measured in a large CsI(TI) crystal calorimeter located inside the $1.5 \mathrm{~T}$ superconducting coil. The energy resolution for photons ( $\sigma_{E}=3.8 \mathrm{MeV}$ for $100 \mathrm{MeV}$ photons) is the best among high energy detectors which operate in this energy range. A fine segmentation (7800 crystals) is important for reconstruction of multi- $\pi^{0}$ final states. Hermeticity of the calorimeter ( $98 \%$ of $4 \pi$ ) proves important in elimination of the background processes. The 51 layers drift chamber inside the calorimeter is used for charged particle tracking. It is supplemented by a vertex detector (smaller drift chamber) and a small straw tube device. In addition to the precise measurement of charged track momenta, the tracking system provides particle identification using $\mathrm{d} E / \mathrm{d} x$ measurement with $6.5 \%$ resolution. This allows for a $K / \pi$ separation up to $0.8 \mathrm{GeV} / c^{2}$, and for a limited $(2 \sigma)$ separation above $2.0 \mathrm{GeV} / c^{2}$. The ToF system based on plastic scintillators located in between the drift chamber and the calorimeter, helps in particle identification at low momenta and is the back-bone of the trigger system. Outside the superconducting coil, an iron filter instrumented with Iarocci tubes at three different depths serves for identification of muons. Upgrade of the CLEO-II tracking system by construction of a silicon strip detector is currently under way. The installation of the silicon detector should be completed later this year. Upgrade of the whole tracking system together with installation of a new particle ID device to improve $K / \pi$ separation is also anticipated for the high luminosity running. The detector with all new components is planned for completion in 1997 (see CLEO-III section).

Since the turn-on of the CLEO-II detector in fall of 1989 the experiment has accumulated data sample corresponding to about $4 \mathrm{fb}^{-1}$ of integrated luminosity. About $2 / 3$ of the data 
Figure 1: 3D-view of the CLEO-II detector.

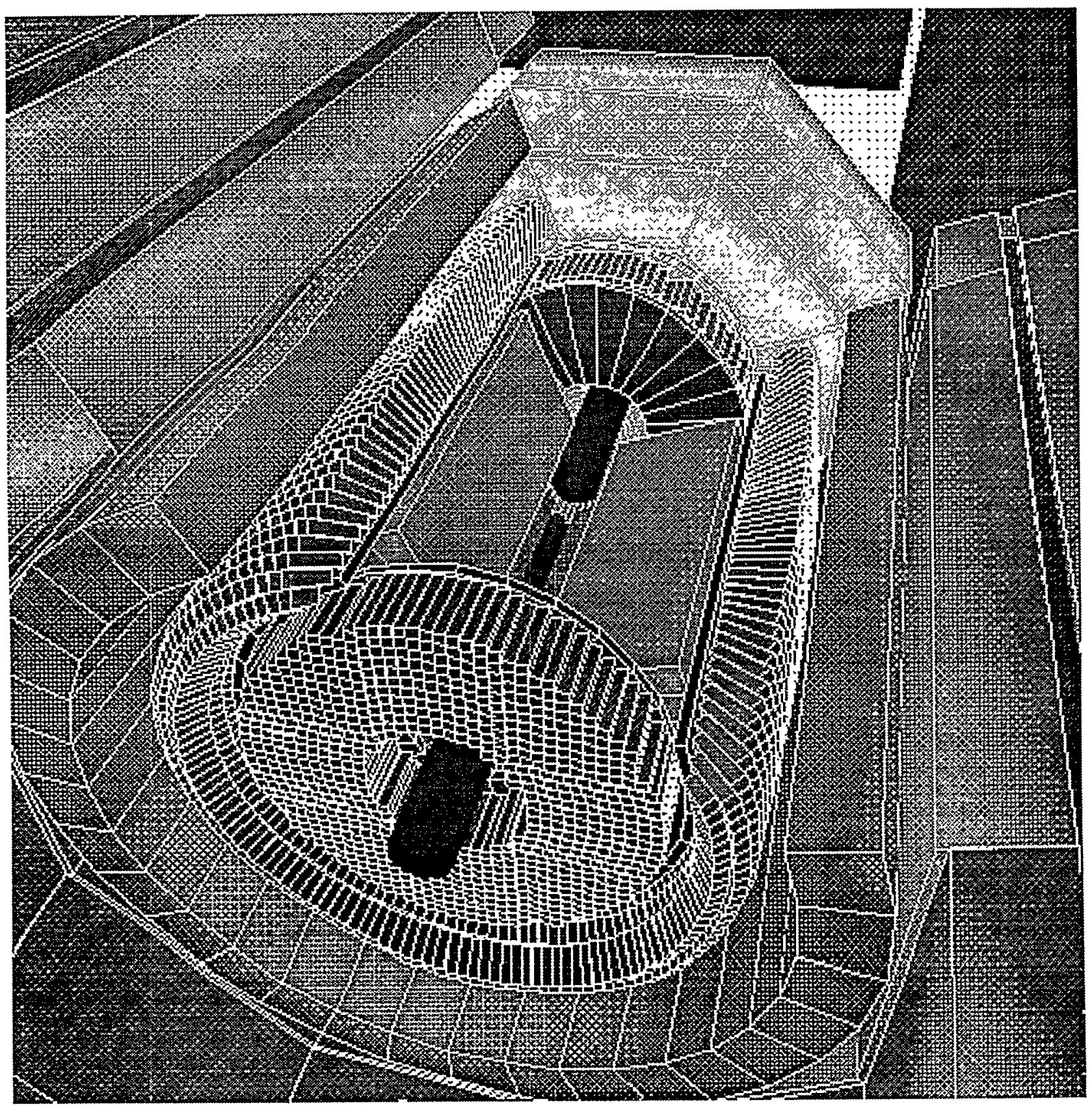


were taken on top of the $\Upsilon(4 S)$ resonance, producing $B \bar{B}$ pairs with cross-section of $1 n b$. The rest of the data were taken mostly at the continuum below the $B \bar{B}$ threshold (used for background subtraction in $B$-physics analyses) and smaller amounts on top of the narrow $\Upsilon$ resonances $\left(60 p b^{-1}\right.$ on top of $\Upsilon(1 S)$ and $120 p b^{-1}$ on top of $\left.\Upsilon(3 S)\right)$. Tau-pairs and $c \bar{c}$ are produced at any beam energy with the cross-section also around $1 n b$ each. Number of $\tau$-leptons recorded by CLEO-II is a factor of 100 larger than in the LEP experiments, and a factor of 10 larger than in the ARGUS experiment. The similar factors have been achieved for $B$ mesons produced in $e^{+} e^{-}$collisions.

These huge statistics of heavy flavors recorded by CLEO-II allowed for significant advances in $B$-meson, $\tau$-lepton, charm and $\Upsilon$ spectroscopy physics. The CLEO-II collaboration has published so far 33 articles in Physical Review Letters, 11 articles in Physical Review $\mathrm{D}$, and 5 articles in Physical Letters B.

In $B$-physics the major achievements were:

- the measurements of effective Flavor Changing Neutral Currents via observation of $b \rightarrow s \gamma$ transitions;

- the first evidence for charmless hadronic decays of $B \rightarrow h^{+} h^{-}(b \rightarrow u W$ and/or $b \rightarrow s g$ transitions);

- the most stringent limits on the other rare $B$ decays $\left(b \rightarrow s l^{+} l^{-}, b \rightarrow d \gamma\right.$, exclusive decay modes for $b \rightarrow s g$ and $b \rightarrow u W)$;

- the most precise measurements of quark mixing elements $V_{c b}$ and $V_{u b}$;

- tests of factorization hypothesis in hadronic decays of $B$-meson achieved by the measurements of branching fractions for a large number of exclusive hadronic and semileptonic decay modes

The CLEO-II has had also a major impact on $\tau$-physics:

- resolution of the "1-prong deficit" problem by precision measurements of exclusive branching fractions for $\tau$-decays into a charged particle and neutral pions;

- improved test of $\tau-\mu$ universality by the most precise measurement of branching fraction for $\tau^{-} \rightarrow \nu_{\tau} e^{-} \bar{\nu}_{e}$;

- the tightest limits on lepton flavor violating decays: $\tau^{-} \rightarrow \mu^{-} \gamma, \tau \rightarrow 3$ charged leptons and to a charged leptons and two hadrons;

- precision measurements of exclusive branching fractions in 3 and 5 charged track topology;

- improved measurements of Cabibbo suppressed decays and decays with two kaons;

- the first observation of $\tau$ decays to $\eta$ and the tightest limit on the branching fraction for the second-class current decay: $\tau^{-} \rightarrow \nu_{\tau} \pi^{-} \eta$; 
- competitive results on $\tau$ lifetime, tau-neutrino mass limit, $\tau$ mass and neutrino helicity in semihadronic decays.

Contributions to charm physics have been equally important:

- Observation of doubly Cabibbo suppressed decays, $D^{0} \rightarrow K^{+} \pi^{-}$;

- Measurement of decay constant of $D_{s}$ in $D_{s} \rightarrow \mu \bar{\nu}_{\mu}$;

- Observation of new charmed baryon and meson states, $\Lambda_{c}^{\star}(2630), D_{s}^{\star}(2573)$;

- Precision measurements of branching ratios for channels used as normalization modes: $D^{0} \rightarrow K^{-} \pi^{+}$and $D^{+} \rightarrow K^{-} \pi^{+} \pi^{+}$

- Measurement of numerous exclusive semileptonic and hadronic branching fractions.

The SMU group has greatly contributed to the success of the CLEO-II experiment. Number of contributions has increased with time in parallel with the expansion of the group. Professor Ryszard Stroynowski moved from Caltech to SMU in summer 1991. Postdoctoral fellow, Sukhpal Sanghera, joined the group in spring 1992 and has been resident in Ithaca since then. Assistant Professor Tomasz Skwarnicki moved from a tenure track position at Syracuse University to SMU in summer 1992. Two new postdoctoral fellows joined the SMU in 1993: Jay Dominick and Mark Lambrecht. Dominick is resident in Dallas, and Lambrecht is resident in Ithaca. Four graduate students joined the group in fall 1992: Vasili Shelkov, Igor Volobouev, Guoheng Wei and Pavel Zadorozhny; and two more in fall 1993: Vitali Fadeyev and Ilya Korolkov. Assistant Professor Tom Coan joined the group in summer 1994.

The main service responsibility of the SMU group since it was admitted to CLEO has been off-line processing of all experimental data with the event reconstruction programs: "PASS2". This is a challenging task considering size and rate of the CLEO-II data, and direct effect on physics analyses performed by the whole collaboration.

The other major contribution of the SMU group has been to provide a leadership role for the analysis of the $\tau$ lepton data. First Stroynowski, more recently Skwarnicki, have chaired the group of about 30 physicist from 6 institutions involved in $\tau$ physics at CLEO.

Members of the SMU group have been principal authors of many important CLEO-II results from $\tau, B$ and QCD physics. Six out of 27 CLEO papers submitted to the Glasgow conference were based on analyses done by the SMU physicists and students.

In the following subsections we detail SMU contributions to the CLEO-II experiment and highlight some of the recent results obtained by us. Activities related to the CLEO-III upgrade of the detector are described in a separate chapter.

\subsection{Data Reconstruction}

The raw CLEO-II data are converted into an analysis-ready format by the event data reconstruction program called PASS2. PASS2 consists of charged track reconstruction, secondary vertex finding, particle identification by specific ionization $(d E / d X)$ and Time-of-Flight 
methods, clustering in the calorimeter, $\pi^{0}$ reconstruction, muon identification, backgrounds filters (for example, beam-wall event killer), event classification and event shape measurements. Once this processing is completed, a separate software package converts the data into a compressed format used in all CLEO physics analyses. At this stage, events of general interest to the CLEO collaboration such as hadronic and $\tau$-pair events are extracted from the full dataset, written to disk and tapes, and distributed to the collaboration.

The computer system used in the reconstruction process consists at present of seven DEC Alpha 3000/600's, five 4mm tape drives, and two 10 Gbyte DLT tape drives. It is divided into the main reconstruction cluster and the hadron and tau event skimming center (see Fig. 2). In addition, approximately 17 Gbytes of disk are dedicated to the project.

The large amount of computing resources is necessary because of the high event rates generated by CESR. Currently, CLEO records approximately one million events per day, including QED processes, annihilation events, $\Upsilon(4 S)$ decays, and beam-gas event. Combined with the steady running conditions and planned upgrades for the accelerator, the magnitude of this job becomes obvious. The reconstruction cluster presently processes slightly more than one million events per day. In more tangible terms, the I/O rate of the reconstruction project is approximately seven Gbytes per day.

When SMU agreed to take over this project in 1991, the process described above was not automated, man-hour intensive, and quite slow. Automation was given a priority and quickly accomplished. The labor force shrunk from six full time people down to two. Currently, the entire project is run by one SMU physicist and a graduate student, new computing resources have been acquired, and processing speed has vastly increased.

Planned upgrades in the CESR luminosity necessitate further improvements in the data reconstruction process. CPU, network, and I/O improvements are all envisioned for the near future, and must be implemented in order to stay abreast of the data acquisition rates and the plans to re-process all the data taken since 1990. Re-processing of all data is planned in order to implement improvements in the reconstruction software that have occurred during the past 4 years. SMU is the sole institution responsible for these improvements and will continue to be in charge of the reconstruction project for the forseeable future.

\subsection{Tau Physics Group}

Work on physics analyses in CLEO is organized into Physics Topic Analysis groups (so called "PTA's"). Each group has its leader who initiates and oversees the physics analyses, organizes work on common tools (e.g. production of Monte Carlo data), serves as liaison with the larger physics community and organizers monthly meetings. Because of extraordinary physics potential of $\tau$ data from the CLEO-II, tau PTA attracted many first class physicist from 6 institutions participating in CLEO-II: Caltech, University of Colorado, Cornell University, University of Kansas, Ohio State University and SMU. Stroynowski co-chaired the Tau PTA with Barry Barish until summer 1993. Skwarnicki has been chairing the Tau PTA since then. The Tau PTA has published 10 papers ( 9 in Phys.Rev.Lett.) during this period. Recently, 8 additional papers on $\tau$ related measurements were submitted to the Glasgow conference. 
Figure 2: (a) Schematic of the computing system used to process the raw data, and (b) system used for extracting hadron and $\tau$-pair events after the reconstruction process.

(a) Data Reconstruction Cluster

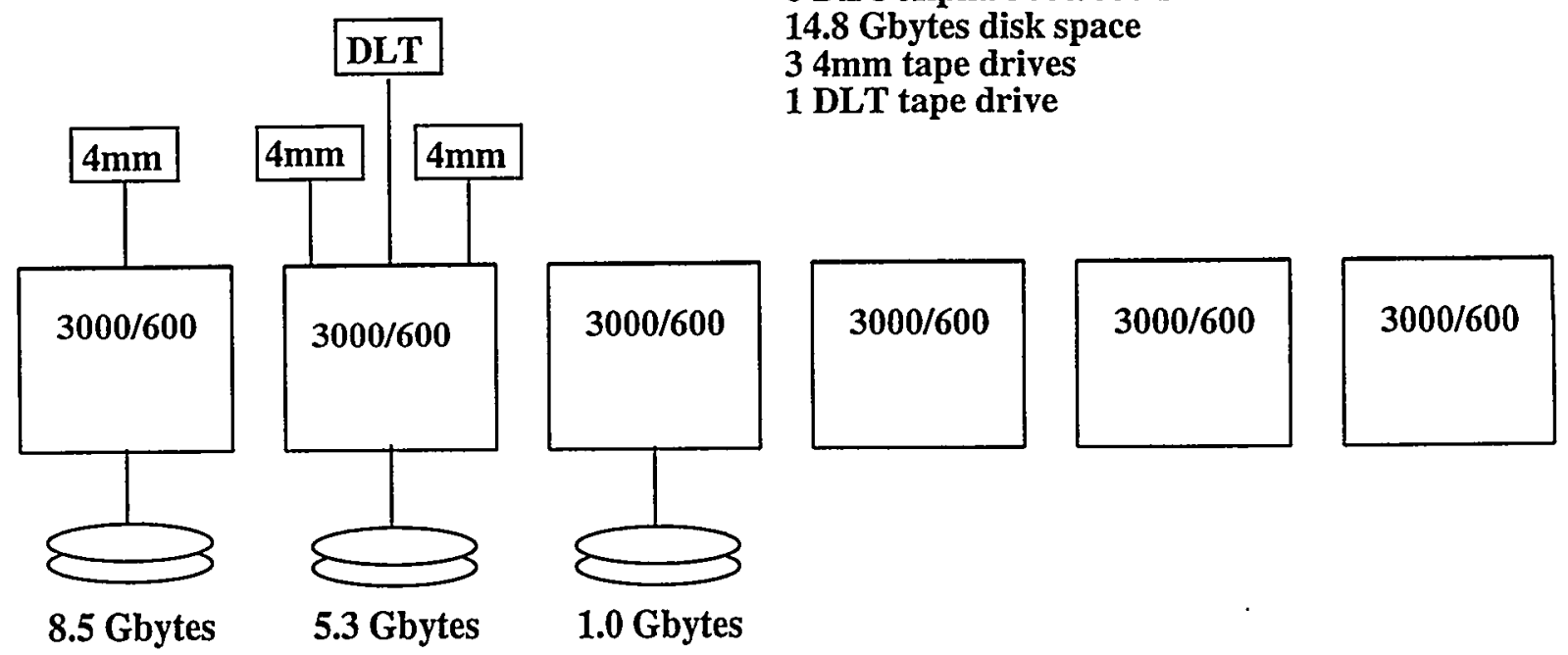

(b) Skimming Computer

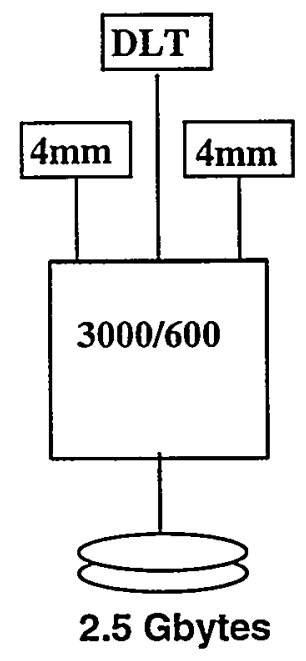

1 DEC Alpha 3000/600

3.8 Gbytes disk space

$24 \mathrm{~mm}$ tape drives

1 DLT tape drive 
Figure 3: Penguin diagram for $b \rightarrow s \gamma$ transitions.

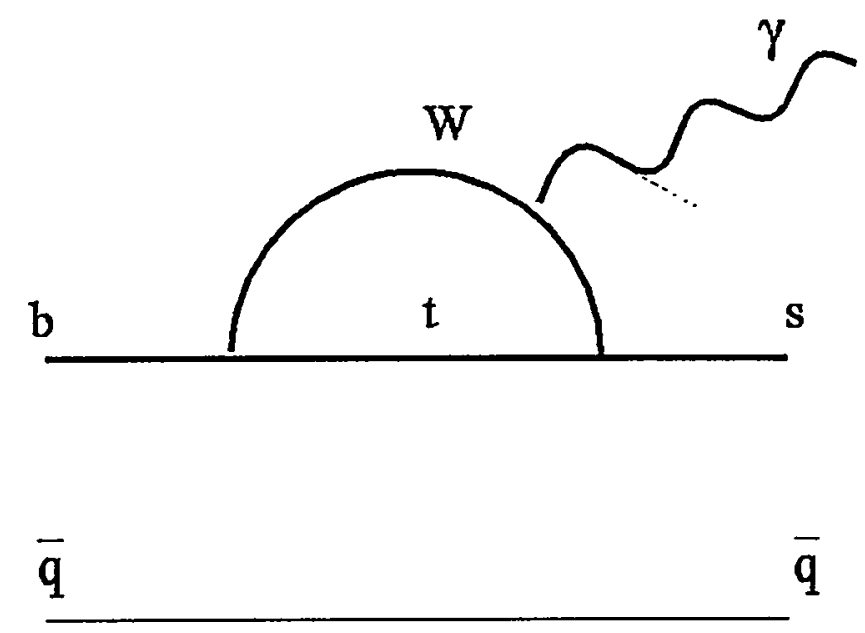

\subsection{Physics Analyses originating at SMU}

\subsubsection{B Physics}

$\underline{\text { Inclusive } \mathrm{b} \rightarrow \mathrm{s} \gamma \text { decays. }}$

The most important recent CLEO result is a measurement of inclusive $b \rightarrow s \gamma$ rate. Skwarnicki has made a very significant contribution to this analysis. The results were first presented by the CLEO collaboration at the Glasgow conference, and represented one of the highlights of this and the other conferences this year.

Since $b \rightarrow s$ quark transitions are Flavor-Changing-Neutral-Currents they are suppressed in the Standard Model at the tree level by the GIM mechanism. They occur in the second order of electroweak interactions via so called loop ("penguin") diagram (see Fig.3). Virtual $W$ is exchanged in a loop with an up type quark - preferentially the top quark. A photon or a gluon is emitted in this process. Such processes are extremely sensitive to physics beyond the Standard Model which may occur at the electroweak scale, since non-standard particles can be exchanged in the loop in addition to the $W$ and top quark. If gluon is emitted, it hadronizes together with the $s$ and the spectator quarks and does not produce clean experimental signature with presently detectable rates. On the other hand if a photon is emitted it can be directly detected in the calorimeter. This provides a clean signature for the $b \rightarrow s \gamma$ decay since the photon is quasi-monochromatic, with energy beyond the endpoint for backgrounds coming from usual $b \rightarrow c$ decays. Last year CLEO-II detected $b \rightarrow s \gamma$ transitions by reconstructing a few $B \rightarrow K^{*} \gamma$ decays for the first time. Although the 
Standard Model predicts the inclusive $b \rightarrow s \gamma$ rate reasonably well, a fraction of the total $b \rightarrow$ $s \gamma$ rate which should show up in the $B \rightarrow K^{*} \gamma$ channel is not well understood (theoretical expectations vary from $1 \%$ to $99 \%$ ). Therefore, a measurement of the inclusive $b \rightarrow s \gamma$ rate is crucial. Experimentally the task is complicated by the large photon background from continuum production of light quark pairs: $e^{+} e^{-} \rightarrow q \bar{q}, q=u, d, s, c$. The continuum background can be suppressed by inclusive reconstruction of $B \rightarrow \gamma K n \pi$ final states. This technique, developed by Skwarnicki, allowed for the observation of $b \rightarrow s \gamma$ signal with statistical significance of 4.4 standard deviations. A complementary neural network approach (developed by the Rochester group) produced less significant (2.8 standard deviations) but consistent results. Inclusive photon spectra obtained from the two techniques are shown in Fig.4-5. The measured inclusive $b \rightarrow s \gamma$ rate, $(2.32 \pm 0.51 \pm 0.43) \cdot 10^{-4}$, agrees well with the Standard Model predictions. This allows to limit the contributions to this process from physics outside of the Standard Model. For example, a lower limit on the charged Higgs mass in the Two-Higgs-Doublet-Model is obtained, $m_{H}>270 \mathrm{GeV}$ at $95 \%$ C.L. for $\tan \beta>2$. This is the most stringent limit on this model available to date. There are many other interesting theoretical consequences of this measurement (see e.g. review by J.L.Hewett, SLAC-PUB-6521 1994).

The inclusive $B$ reconstruction technique which we developed for this analysis holds the promise of improvement in searches for other penguin processes, e.g. $b \rightarrow s l^{+} l^{-}$or $B \rightarrow \phi X$ (the later is a footprint for $b \rightarrow$ sgluon decays). We are planning to pursue this path in the future. The expected increase of the CLEO-II data will improve statistical error on the measured $b \rightarrow s \gamma$ rate. In more distant future, new particle ID device to be installed in the CLEO-III detector upgrade will significantly reduce continuum background under the $b \rightarrow s \gamma$ signal by better identification of the strangeness of the hadronic system recoiling against the photon.

\section{Semileptonic decays of B.}

The $B$ semileptonic branching fractions offer a testing ground for the electro-weak interaction. In semileptonic decays, $W$ emitted in $b \rightarrow c$ transitions turns to a lepton and its neutrino. Thus theoretical predictions must account for strong interactions between only three quarks $(b, c$, and the spectator quark $u$ or $d)$, as opposed to five quarks in purely hadronic decays. As a consequence this amplitude is easier to calculate. Naive counting of quarks and leptons (in the absence of QCD effects) leads to a branching fraction of $\sim 17 \%$. More careful, second order QCD calculations predict $\operatorname{Br}(B \rightarrow X \ell \nu) \geq 12.5 \%$. All experimental measurements of the average charged and neutral $B$ semileptonic branching fractions are significantly lower than this prediction, and are in the range of $10 \%-11 \%$. Possible explanations for the discrepancy between theory and experiment include deviation from the two assumptions made in the measurements: that the $\Upsilon(4 S)$ decays in $100 \%$ to $B \bar{B}$, and that the rates of the decays to $B^{0} \bar{B}^{0}\left(f_{00}\right)$ and to $B^{+} B^{-}\left(f_{+-}\right)$are equal. If the first assumption is invalid, the total number of $B$ 's in the data sample would be over-estimated, resulting in a branching fraction that is too small. If the charged and neutral $B$ branching fractions are not 


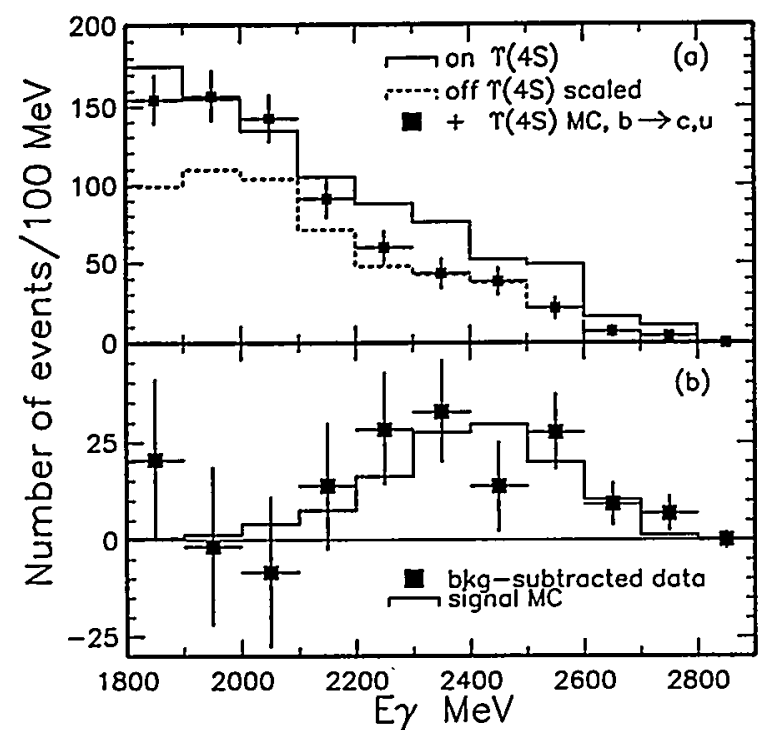

Figure 4: Photon energy spectra from the inclusive $B$ reconstruction technique. (a) On- resonance data is indicated by the solid histogram, the scaled off-resonance data is indicated by the dashed histogram, and the points with the error bars illustrate total estimated background which in addition to the continuum includes $B$ decay backgrounds estimated by the Monte Carlo simulation. Excess of the solid histogram over the points with the error bars is an evidence for $b \rightarrow s \gamma$ decays. (b) Background subtracted signal distribution (points with error bars) compared to the Monte Carlo simulation (solid line).

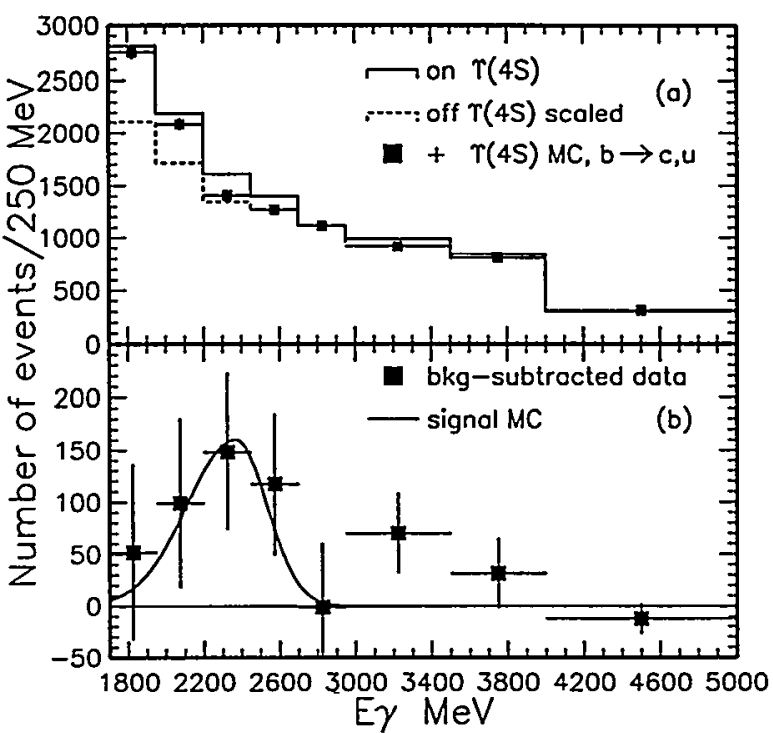

Figure 5: Photon energy spectra from the neural net technique. Symbols are defined in the previous caption. 
equal, and the second assumption is wrong, what is being measured is not the true average of branching fractions, but rather a ratio skewed toward one or the other $B$-meson flavor. In order to eliminate the need for these poorly tested assumptions, a method of tagging a $\bar{B}$ in the event and examining the decay products of the other $B$ meson for its lepton content has been developed. Once a $\bar{B}$ is tagged, non $\Upsilon(4 S) \rightarrow B \bar{B}$ decays are eliminated from the data sample and the flavor of the $B$ decaying to leptons is known. In this manner, separate measurements of the semileptonic decay branching fractions of $B^{+}$and $B^{0}$ can be made. We will denote them by $b_{+} \equiv \operatorname{Br}\left(B^{+} \rightarrow X \ell \nu\right)$ and $b_{0} \equiv \operatorname{Br}\left(B^{0} \rightarrow X \ell \nu\right)$. The former branching fraction has not been measured previously. If there is no asymmetry in the semileptonic partial widths, the ratio of $b_{+}$to $b_{0}$ is equivalent to the ratio of the $B_{d}$ and $B_{u}$ lifetimes. This measurement of $\frac{\tau_{+}}{\tau_{0}}$ is the first measurement at $B \vec{B}$ threshold machines that is independent of the charge and neutral $B$ production fractions (the second assumption listed above).

Decays of $B$ are tagged using three techniques: (A) full reconstruction of all decay products, (B) partial reconstruction of a semileptonic decay mode, and (C) partial reconstruction of a hadronic channel. Lambrecht has assumed responsibility for Tag $(A)$, which gives the only measurement of $b_{+}$, while all three tags yield numbers for $b_{0}$. Eight distinct decay modes are reconstructed in this method: $D \pi^{-}, D^{*} \pi^{-}, D \rho^{-}, D^{*} \rho^{-}, D a_{1}^{-}, D^{*} a_{1}^{-}, \psi K$ and $\psi K^{*}$, with charm mesons in the channels:

$$
\begin{aligned}
& D^{*+} \rightarrow D^{0} \pi^{+}, D^{+} \pi^{0} \\
& D^{* 0} \rightarrow D^{0} \pi^{0} \\
& D^{0} \rightarrow K^{-} \pi^{+}, K^{-} \pi^{+} \pi^{0}, K^{-} \pi^{+} \pi^{+} \pi^{-}, K_{\mathrm{S}}^{0} \pi^{0}, K_{\mathrm{S}}^{0} \pi^{+} \pi^{-} \\
& D^{+} \rightarrow K^{-} \pi^{+} \pi^{+}, K_{\mathrm{S}}^{0} \pi^{+} \\
& J / \psi \rightarrow e^{+} e^{-}, \mu^{+} \mu^{-}
\end{aligned}
$$

and light mesons reconstructed in the modes $\pi^{0} \rightarrow \gamma \gamma, K_{\mathrm{S}}^{0} \rightarrow \pi^{+} \pi^{-}, K^{*+} \rightarrow K^{+} \pi^{0}, K^{*+} \rightarrow K_{\mathrm{S}}^{0} \pi^{+}$, $K^{* 0} \rightarrow K_{\mathrm{S}}^{0} \pi^{0}, K^{* 0} \rightarrow K^{+} \pi^{-}, \rho^{+} \rightarrow \pi^{0} \pi^{+}, \rho^{0} \rightarrow \pi^{+} \pi^{-}$and $a_{1}^{+} \rightarrow \rho^{0} \pi^{+}$. The above illustrates complexity of the work done by Lambrecht.

Once a $B$-meson has been tagged, the remaining charged tracks in the event are examined for electron or muon content. A momentum of $p(\ell)>1.4 \mathrm{GeV} / \mathrm{c}$ was required to suppress leptons from charmed mesons. The semileptonic branching fractions are then given by: $b_{+}=$ $N_{\text {leptons }} / N_{B^{+} \text {tags }}$ and $b_{0}=N_{\text {leptons }} / N_{B^{0} \text { tags }}$ where $N_{\text {leptons }}$ refers to the efficiency corrected number of leptons found opposite the $B^{+}$or $B^{0}$ tags.

Figure 6 shows the distribution of tags and the subset of those tagged events containing leptons from the other $B$ in the event for tags (A) and (B). Superimposed are the background estimates used to obtain the signal amplitudes.

Using a weighted average for the neutral $B$ results, we find

$$
\begin{aligned}
b_{+} & =(10.1 \pm 1.8 \pm 1.4) \%, \\
b_{0} & =(10.9 \pm 0.7 \pm 1.1) \% .
\end{aligned}
$$

Both are consistent with the CLEO II average inclusive branching fraction, (10.9 $\pm 0.1 \pm 0.3) \%$.

Assuming equal charged and neutral partial semileptonic decay widths, the lifetime ratio is given by the ratio of semileptonic branching fractions:

$$
\frac{\tau_{+}}{\tau_{0}}=\frac{b_{+}}{b_{0}}=0.93 \pm 0.18 \pm 0.12
$$


Figure 6: Samples of tagged $B$ mesons, without (a) and with additional lepton required (b).

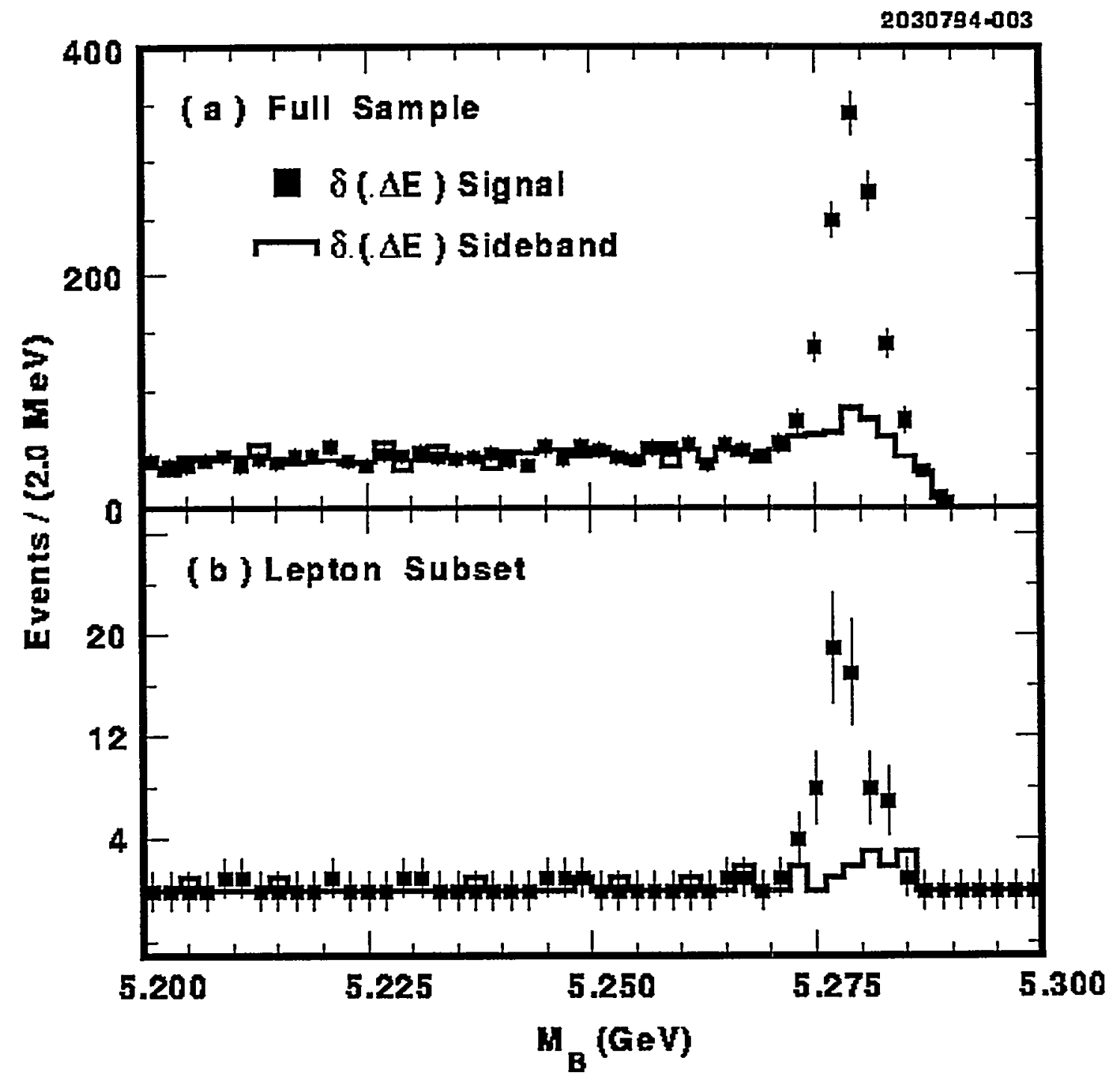


This result is in agreement with both earlier CLEO and ARGUS measurements, which assume that $f_{+-} / f_{00}=1$. It is consistent with the world average lifetime ratio of $1.10 \pm 0.11$ as well as with theoretical expectations, and is of comparable significance to existing individual measurements.

The above results have been submitted for a publication.

Increased statistics of the future CLEO-II sample will allow to refine the above results.

\section{A Search for Semileptonic B Decays to Charmed Baryons.}

The $B$-meson is the only meson which has been observed to produce baryons in its decay. The mechanism for this production is, however, poorly understood due to lack of experimental statistics. Previous studies have assumed that an external $W$-emission model $\left(\bar{B} \rightarrow \Lambda_{c} \bar{N} W_{e x t}\right)$ is the dominant baryon production mechanism in $B$ decays. In this picture, baryons are produced via the "popping" of two quark-antiquark pairs from the vacuum. These bind to form a $\Lambda_{c}$ and an antinucleon $\bar{N}$ at the lower vertex, while the external $W$ hadronizes or decays to a lepton-neutrino pair. In principle, however, internal $W$-emission could account for a substantial fraction of the baryons produced in $B$-decay. In contrast to the mesonic sector, in which internal $W$-emission is suppressed by color matching of the quarks, the color degrees of freedom of the "popped" $q \bar{q}$ in baryon production should eliminate any such suppression. This mechanism may in fact be favored due to the need to "pop" only one $q \bar{q}$ pair from the vacuum.

Semileptonic $B$-decays to baryons may be used to qualitatively distinguish between the external and internal spectator mechanisms, since they occur only via external $W$-emission. No evidence for such decays would point to an enhancement in the internal diagram. This is attractive from the standpoint of reducing the semileptonic branching ratio owing to the larger hadronic fraction of the total $B$-decay width. This then becomes a possible rationale for the unexpectedly small semileptonic $B$-branching fraction of $10.5 \%$ compared with theory, which can accommodate values only as low as $12.5 \%$. If, by contrast, the $\bar{B} \rightarrow \Lambda_{c} \bar{N} W_{\text {ext }}$ mechanism dominates, then the decay $\bar{B} \rightarrow \Lambda_{c} \bar{N} X \ell \nu$ should occur, much as the mesonic decay $\bar{B} \rightarrow D X \ell \nu$ occurs. Neglecting the penalty required to pop two $q \bar{q}$ pairs from the vacuum, then one might anticipate:

$$
\frac{\vec{B} \rightarrow D X \ell \nu_{\ell}}{\bar{B} \rightarrow D X} \sim \frac{\bar{B} \rightarrow \Lambda_{c} X \ell \nu_{\ell}}{\bar{B} \rightarrow \Lambda_{c} X} .
$$

In fact, the large mass of the $\Lambda_{c} \bar{N}$ system will introduce a severe phase space suppression for the hadronic decay $W_{\text {external }} \rightarrow \bar{c} s$ so that the fractional semileptonic rate, if anything, should be larger in the case of charmed baryon compared to charmed meson production. Other factors such as interference between internal and external spectator decays in the mesonic sector contribute to make this comparison less exact, but a reduced $B \rightarrow$ baryons semileptonic rate would indicate that the internal spectator mechanism is more important than has been previously assumed. If this is the case, absolute branching fractions for decays 


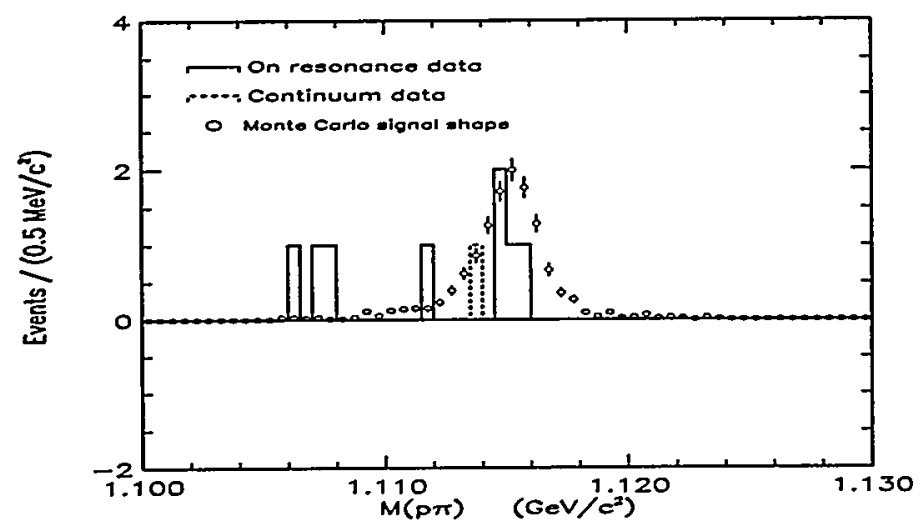

Figure 7: $\Lambda \mathrm{E}_{\text {tag }} \mathrm{E}_{\text {signal }}$ candidates displayed as the invariant mass of the $\Lambda$.

such as $\Lambda_{c} \rightarrow p K \pi$ as well as inclusive rates for channels like $\Lambda_{c} \rightarrow \Lambda X$ will have to be reevaluated.

Lambrecht initiated analysis of the data to address the above outlined problem. In order to measure the branching fraction for the decay $\vec{B} \rightarrow \Lambda_{c} X \ell \nu$, we search for $\Lambda \ell$ correlations kinematically consistent with the decay of interest. Here, $\Lambda_{c} \rightarrow \Lambda X$. Uncorrelated $\Lambda \ell$ pairs (from the continuum, for example) form a large source of background which can be largely eliminated by tagging the flavor of the other $B$ using hard primary leptons. Thus the signature of interest is $\left(\Lambda \ell^{-}\right.$signal $) \ell_{\text {tag. }}^{+} . \Lambda^{\prime}$ 's are reconstructed in the decay channel $\Lambda \rightarrow p \pi^{-}$ by searching for two intersecting tracks displaced from the primary vertex. Both the pion and the proton must have measured ionization losses of that expected for the given mass hypothesis.

Candidate events passing all selection criteria are then examined via the $\Lambda$ invariant mass plot. Fig. 7 shows this distribution for the on- and off-resonance data, as well as a Monte Carlo simulation of the $\Lambda$ mass. To be considered as a possible signal, candidates must lie in the mass range $1.11263<M(p \pi)<1.11863 \mathrm{GeV} / \mathrm{c}^{2}$. There are four (one) such candidates from the on (off) resonance data. This is consistent with the expected background rates estimated from the Monte Carlo simulation.

Since no signal is observed an upper limit on the branching fraction of the decay $B \rightarrow$ $\Lambda_{c} \ell \nu X$ is obtained: $\mathcal{B}\left(B \rightarrow \Lambda_{c} \ell \nu X\right) / \mathcal{B}\left(B \rightarrow \Lambda_{c} X\right)<5.7 \%, 90 \%$ C.L.. The equivalent ratio in $B \rightarrow D$ decays, summing over charged and neural $D$ 's, is $\sim 12 \%$. The much lower value found in the baryon sector points toward internal $W$-emission decays playing a much larger role than in the meson sector. In addition, the upper limit of $\mathcal{B}\left(B \rightarrow \Lambda_{c} X \ell \nu\right)<0.72 \%$ rules out the possibility of this decay contributing to the discrepancy between the theoretical predictions and the experimental measurements of the fraction $\mathcal{B}(B \rightarrow X \ell \nu)$. 
The results are preliminary.

\subsubsection{Tau Physics}

$\underline{\text { Search for flavor violating decays }}$

There is no fundamental motivation for lepton flavor conservation in the Standard Model since there is no symmetry associated with lepton family number. Therefore, searches for the lepton flavor changing processes probe basic assumptions of the theory. Many extensions of the Standard Model predict lepton flavor violation; these extensions are constrained by strict limits on neutrinoless muon decays: $\mathcal{B}(\mu \rightarrow e \gamma)<4.9 \cdot 10^{-11}$ and $\mathcal{B}(\mu \rightarrow e e e)<2.4 \cdot 10^{-12}$ at $90 \% \mathrm{CL}$. In some of the models there is a large enhancement of the rate of neutrinoless tau decays due to mass-dependent couplings of the proposed new physics processes.

The SMU group (Volobouev under supervision of Stroynowski and Skwarnicki) has search for tau decays into three charged leptons:

$$
\begin{array}{ll}
\tau^{-} \rightarrow e^{-} e^{+} e^{-}, & \tau^{-} \rightarrow e^{-} e^{-} \mu^{+}, \\
\tau^{-} \rightarrow e^{-} e^{+} \mu^{-}, & \tau^{-} \rightarrow e^{-} \mu^{+} \mu^{-}, \\
\tau^{-} \rightarrow e^{+} \mu^{-} \mu^{-}, & \tau^{-} \rightarrow \mu^{-} \mu^{+} \mu^{-},
\end{array}
$$

together with a search for tau decays into one charged lepton and two charged hadrons (pions or kaons):

$$
\begin{array}{ll}
\tau^{-} \rightarrow e^{-} \pi^{+} \pi^{-}, & \tau^{-} \rightarrow \mu^{-} \pi^{+} \pi^{-}, \\
\tau^{-} \rightarrow e^{-} \pi^{-} K^{+}, & \tau^{-} \rightarrow \mu^{-} \pi^{-} K^{+}, \\
\tau^{-} \rightarrow e^{-} \pi^{+} K^{-}, & \tau^{-} \rightarrow \mu^{-} \pi^{+} K^{-}, \\
\tau^{-} \rightarrow e^{+} \pi^{-} \pi^{-}, & \tau^{-} \rightarrow \mu^{+} \pi^{-} \pi^{-}, \\
\tau^{-} \rightarrow e^{+} \pi^{-} K^{-}, & \tau^{-} \rightarrow \mu^{+} \pi^{-} K^{-} .
\end{array}
$$

We also have searched for tau decays into one charged lepton and a neutral meson which decays subsequently into two charged hadrons:

$$
\begin{array}{ll}
\tau^{-} \rightarrow e^{-} \rho^{0}, & \tau^{-} \rightarrow \mu^{-} \rho^{0}, \\
\tau^{-} \rightarrow e^{-} K^{* 0}, & \tau^{-} \rightarrow \mu^{-} K^{* 0}, \\
\tau^{-} \rightarrow e^{-} \bar{K}^{* 0}, & \tau^{-} \rightarrow \mu^{-} \bar{K}^{* 0} .
\end{array}
$$

Charge conjugate reactions are also searched for in all 22 channels.

We search for events where one $\tau$ decays into a single charged particle (1-prong decay) and the other $\tau$ decays into three charged particles (3-prong decay). The 3-prong decay is a signal candidate and the 1-prong decay is an allowed $\tau$ decay with one charged particle, zero or more photons and at least one neutrino in the final state. For a neutrinoless $\tau$ decay the total energy measured on the 3 -prong side, $E_{3}$, must be equal to the beam energy, $E_{\text {beam }}$, and the invariant mass of the three charged particles, $M_{3}$, must be equal to the $\tau$ mass. For each channel we consider a rectangular signal region in the $E_{3}-E_{\text {beam }}$ and $M_{3}$ variables. 
The size of this signal region depends on the resolution of the invariant mass and energy measurements and, therefore, varies for different channels. In particular, final state radiation degrades the momentum resolution for electrons, affecting the size of the signal region. The signal region range is also sensitive to the background level. The 3-prong invariant mass distributions of events passing all selection criteria and lying inside the $E_{3}-E_{\text {beam }}$ limits are shown in Fig. 8, together with the expected signal shapes generated by the Monte Carlo. Within the signal region, there is one event in the $\tau^{-} \rightarrow e^{-} \pi^{+} K^{-}$channel which is also a candidate for $\tau^{-} \rightarrow e^{-} \bar{K}^{* 0}$. There is one event in the $\tau^{-} \rightarrow \mu^{-} \pi^{-} K^{+}$channel and two events in the $\tau^{-} \rightarrow \mu^{+} \pi^{-} K^{-}$channel. The number of observed events is consistent with the estimated background due to hadron misidentification as an electron or a muon. No candidates are found in all other channels. The final limits are summarized in Table 1. For comparison, the table also shows the most restrictive upper limits obtained by previous experiments. The results are several times more stringent than those obtained previously. In addition, the limit on $\mathcal{B}(\tau \rightarrow e e e)$ is the most stringent limit to date on lepton flavor violation in $\tau$ decays.

The results were contributed to the Glasgow conference and were submitted to Phys.Rev.Lett.

Precision measurement of 3 -prong branching fractions.

Shelkov under supervision of Skwarnicki have measured the branching fractions for of $\mathcal{B}\left(\tau^{-} \rightarrow h^{-} h^{+} h^{-}\left(\pi^{0}\right) \nu_{\tau}\right)$, where $h$ refers to either a $\pi$ or $K$. An accurate determination of the branching fraction for the decay $\tau \rightarrow 3 h^{ \pm} \nu_{\tau}$ is of particular importance since there is a wide variation among previous measurements. Several previous experiments found a small branching fraction, notably the ARGUS experiment, which obtained $\mathcal{B}(3 h)=0.073 \pm 0.001 \pm$ 0.005. On the other hand, a preliminary ALEPH measurement, $\mathcal{B}(3 h)=0.0957 \pm 0.0024 \pm$ 0.0022 , is nearly four standard deviations larger than the ARGUS result. The CLEO analysis aimed at resolving this discrepancy.

The decay $\tau \rightarrow 3 h^{ \pm} \pi^{\circ} \nu_{\tau}$ is also of considerable interest, partly because it is the second largest 3-prong $\tau$ decay. Though previous measurements for this mode have generally been in reasonable agreement, they are not very precise. Also most of these measurements used photon counting rather than $\pi^{\circ}$ reconstruction technique available at CLEO and thus were more sensitive to feed-across backgrounds.

We measure these decay modes in a two independent ways. First, we use a conventional lepton-tag analysis, taking advantage of the excellent electron and muon identification of the CLEO-II detector. This method has the advantage that the background from $q \bar{q}$ events $\left(e^{+} e^{-} \rightarrow q \bar{q}, q=u d s c\right)$ is quite low. The second approach selects a sample of events where both $\tau$ 's decay to three charged tracks (to be referred to as the 3-3 sample). This method has not been used previously and is possible only with a very large data sample. There are two main advantages: many systematic errors are reduced by a factor of two since one measures the square of the branching fraction; and the technique does not depend directly on the knowledge of any other $\tau$ decay modes (though the estimation of the amount of "feed-across" background from other $\tau$ decay modes does). The only disadvantage is that 


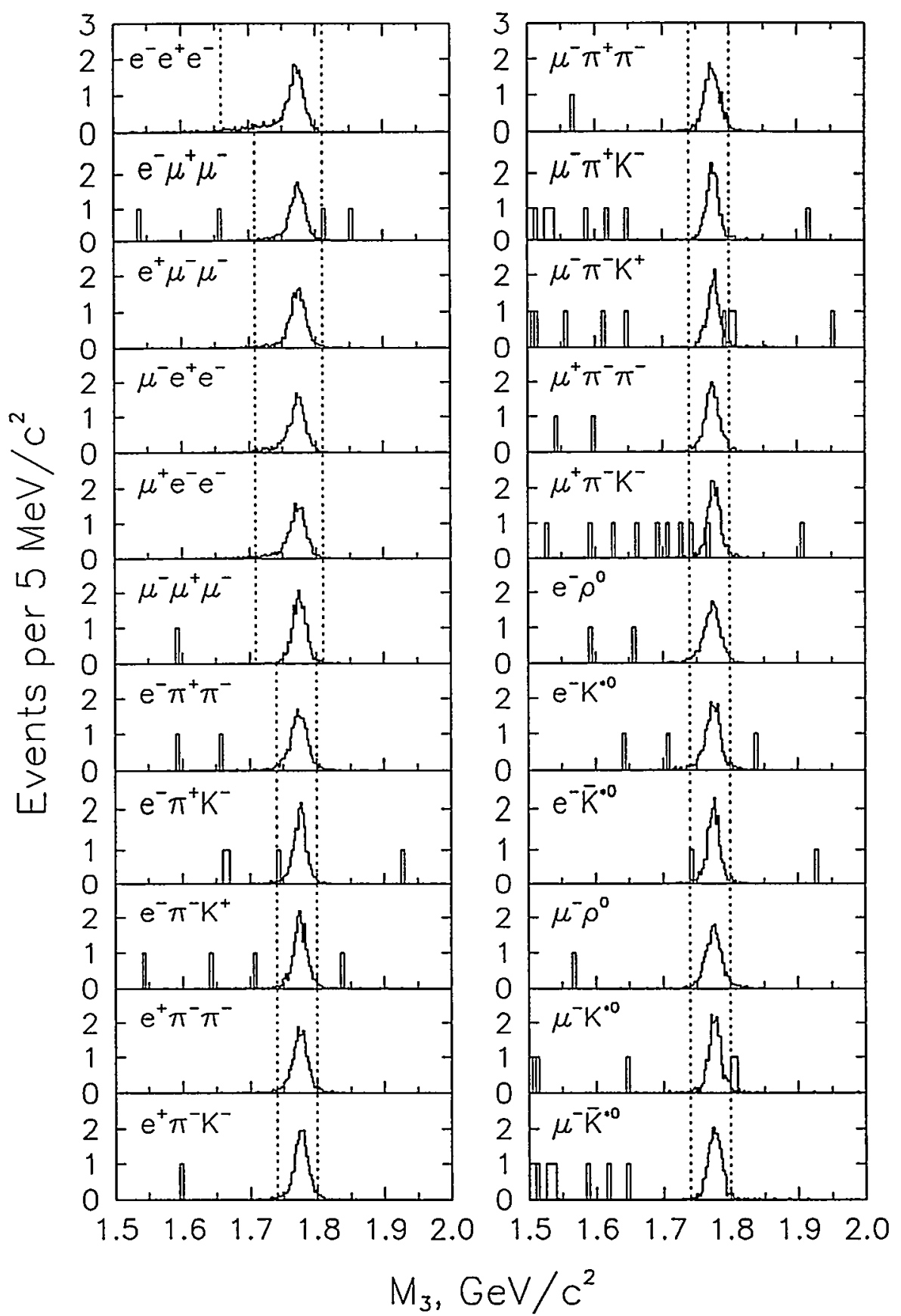

Figure 8: Distributions of the invariant mass of the 3-prong side particles, $\mathrm{M}_{3}$. The expected signal shapes are shown with arbitrary normalization. The dotted lines indicate the boundaries of the signal regions used. 
Table 1: The detection efficiencies, $\epsilon$, and upper limits for the branching fractions at $90 \%$ $\mathrm{CL}, \mathcal{B}$, together with the most restrictive limits from previous experiments.

\begin{tabular}{|c|c|c|c|}
\hline \multirow{2}{*}{$\begin{array}{l}\text { Decay } \\
\text { channel }\end{array}$} & \multirow[t]{2}{*}{$\epsilon(\%)$} & \multicolumn{2}{|c|}{$\mathcal{B}$ (in units of $10^{-6}$ ) } \\
\hline & & Previous & This analysis \\
\hline$\overline{\tau^{-} \rightarrow e^{-} e^{+} e^{-}}$ & 20.4 & 13 & 3.3 \\
\hline$\tau^{-} \rightarrow \mu^{-} e^{+} e^{-}$ & 19.6 & 14 & 3.4 \\
\hline$\tau^{-} \rightarrow \mu^{+} e^{-} e^{-}$ & 19.9 & 14 & 3.4 \\
\hline$\tau^{-} \rightarrow e^{-} \mu^{+} \mu^{-}$ & 18.8 & 19 & 3.6 \\
\hline$\tau^{-} \rightarrow e^{+} \mu^{-} \mu^{-}$ & 19.4 & 16 & 3.5 \\
\hline$\tau^{-} \rightarrow \mu^{-} \mu^{+} \mu^{-}$ & 15.9 & 17 & 4.3 \\
\hline$\tau^{-} \rightarrow e^{-} \pi^{+} \pi^{-}$ & 15.5 & 27 & 4.4 \\
\hline$\tau^{-} \rightarrow e^{-} \pi^{-} K^{+}$ & 14.6 & 58 & 4.6 \\
\hline$\tau^{-} \rightarrow e^{-} \pi^{+} K^{-}$ & 14.9 & 29 & 7.7 \\
\hline$\tau^{-} \rightarrow e^{+} \pi^{-} \pi^{-}$ & 15.5 & 17 & 4.4 \\
\hline$\tau^{-} \rightarrow e^{+} \pi^{-} K^{-}$ & 15.1 & 20 & 4.5 \\
\hline$\tau^{-} \rightarrow \mu^{-} \pi^{+} \pi^{-}$ & 9.1 & 36 & 7.4 \\
\hline$\tau^{-} \rightarrow \mu^{-} \pi^{-} K^{+}$ & 7.4 & 77 & 15 \\
\hline$\tau^{-} \rightarrow \mu^{-} \pi^{+} K^{-}$ & 7.8 & 77 & 8.7 \\
\hline$\tau^{-} \rightarrow \mu^{+} \pi^{-} \pi^{-}$ & 9.8 & 39 & 6.9 \\
\hline$\tau^{-} \rightarrow \mu^{+} \pi^{-} K^{-}$ & 7.7 & 40 & 20 \\
\hline$\tau^{-} \rightarrow e^{-} \rho^{0}$ & 16.2 & 19 & 4.2 \\
\hline$\tau^{-} \rightarrow e^{-} K^{* 0}$ & 10.7 & 38 & 6.3 \\
\hline$\tau^{-} \rightarrow e^{-\bar{K}^{* 0}}$ & 10.5 & - & 11 \\
\hline$\tau^{-} \rightarrow \mu^{-} \rho^{0}$ & 11.9 & 29 & 5.7 \\
\hline$\tau^{-} \rightarrow \mu^{-} K^{* 0}$ & 7.2 & 45 & 9.4 \\
\hline$\tau^{-} \rightarrow \mu^{-} \bar{K}^{* 0}$ & 7.8 & - & 8.7 \\
\hline
\end{tabular}


the hadronic background is larger though this is not a serious limitation. Both methods require precise knowledge of the luminosity and $\tau$-pair production cross section. We use both methods to determine the branching fraction for the $\tau \rightarrow 3 h^{ \pm} \nu_{\tau}$ decay mode ( $3 h$ analysis), but only the lepton-tag method for the $\tau \rightarrow 3 h^{ \pm} \pi^{\circ} \nu_{\tau}$ decay ( $3 h \pi^{\circ}$ analysis). SMU has performed the lepton tagged analysis.

We have measured

$$
\begin{aligned}
\mathcal{B}\left(\tau \rightarrow 3 h^{ \pm} \nu_{\tau}\right) & =0.0982 \pm 0.0009 \pm 0.0034 \text { and } \\
\mathcal{B}\left(\tau \rightarrow 3 h^{ \pm} \pi^{\circ} \nu_{\tau}\right) & =0.0425 \pm 0.0009 \pm 0.0026
\end{aligned}
$$

In Fig. 9, we compare the first result with the older published measurements and recent LEP results. The shaded band represents the one-standard-deviation uncertainty for the new world average obtained by combining this measurement, the two preliminary LEP results, and the measurements in PDG. Since the $\chi^{2}$ is 29.2 for the nine measurements, the uncertainty has been inflated by $\sqrt{\chi^{2} / \mathrm{DOF}}$. Our preliminary result for the $3 h$ decay is in agreement with, but larger than, most previous measurements. We disagree with the ARGUS and DELPHI results at the $4.1 \sigma$ and $2.7 \sigma$ levels, respectively; the former discrepancy is particularly severe since both the ARGUS and CLEO measurements are dominated by systematic errors. Our measurement for the $3 h \pi^{\circ}$ decay is more precise and smaller than most previous measurements as shown in Fig. 10 The agreement is better for this mode, where the $\chi^{2}$ is 11.5 for the eight measurements; nonetheless the errors have been increased as above. Previously only the ARGUS and ALEPH results have reconstructed the $\pi^{\circ}$ in the signal events; our result is in agreement with these measurements. From these results and the previous CLEO measurement for the $3 h 2 \pi^{\circ}$ final state, we determine the 3 -prong branching fraction to be $B_{3}=0.1455 \pm 0.0013 \pm 0.0059$.

The results were presented at the Glasgow conference.

Tau decays to $\nu_{\tau} \mathrm{K}_{\mathrm{S}}^{0} \mathrm{~K}\left(\pi^{\circ}\right)$.

Decays of the $\tau^{-}$to final states containing kaons provide information on both the SU(3) symmetry properties of the charged weak current, as well as rare decays of $\rho^{-}$and $a_{1}^{-}$to strange final states. Dominick has contributed analysis of decays of tau leptons to one neutral $\mathrm{K}$ meson and one charged $\mathrm{K}$ meson. We have measured the branching ratios of the decays $\tau^{-} \rightarrow K^{\circ} K^{-} \nu_{\tau}$ and $\tau^{-} \rightarrow K^{\circ} K^{-} \pi^{\circ} \nu_{\tau}$ using a sample of tau pair events selected with a 1 charged track vs. 3 charged track topology.

Theoretical calculations for the processes governing these decays are limited. For example, expectations for $\tau^{-} \rightarrow K^{\circ} K^{-} \nu_{\tau}$ are on the order of $10^{-3}$. Eidelman and Ivanchenko use existing data for $e^{+} e^{-} \rightarrow \pi^{+} \pi^{-}$and invoke conservation of the vector current (CVC) to obtain a prediction for $\tau^{-} \rightarrow K^{\circ} K^{-} \nu_{\tau}$; this approach has been very successful in matching the measured tau-decay rates for modes without kaons. Taking into account SU(3)-breaking effects such as the $\pi-K$ mass difference, they determine an expected branching ratio for $\tau^{-} \rightarrow K^{\circ} K^{-} \nu_{\tau}$ of $0.11 \pm 0.03 \%$ based on this method. Using a similar calculation, Narison 


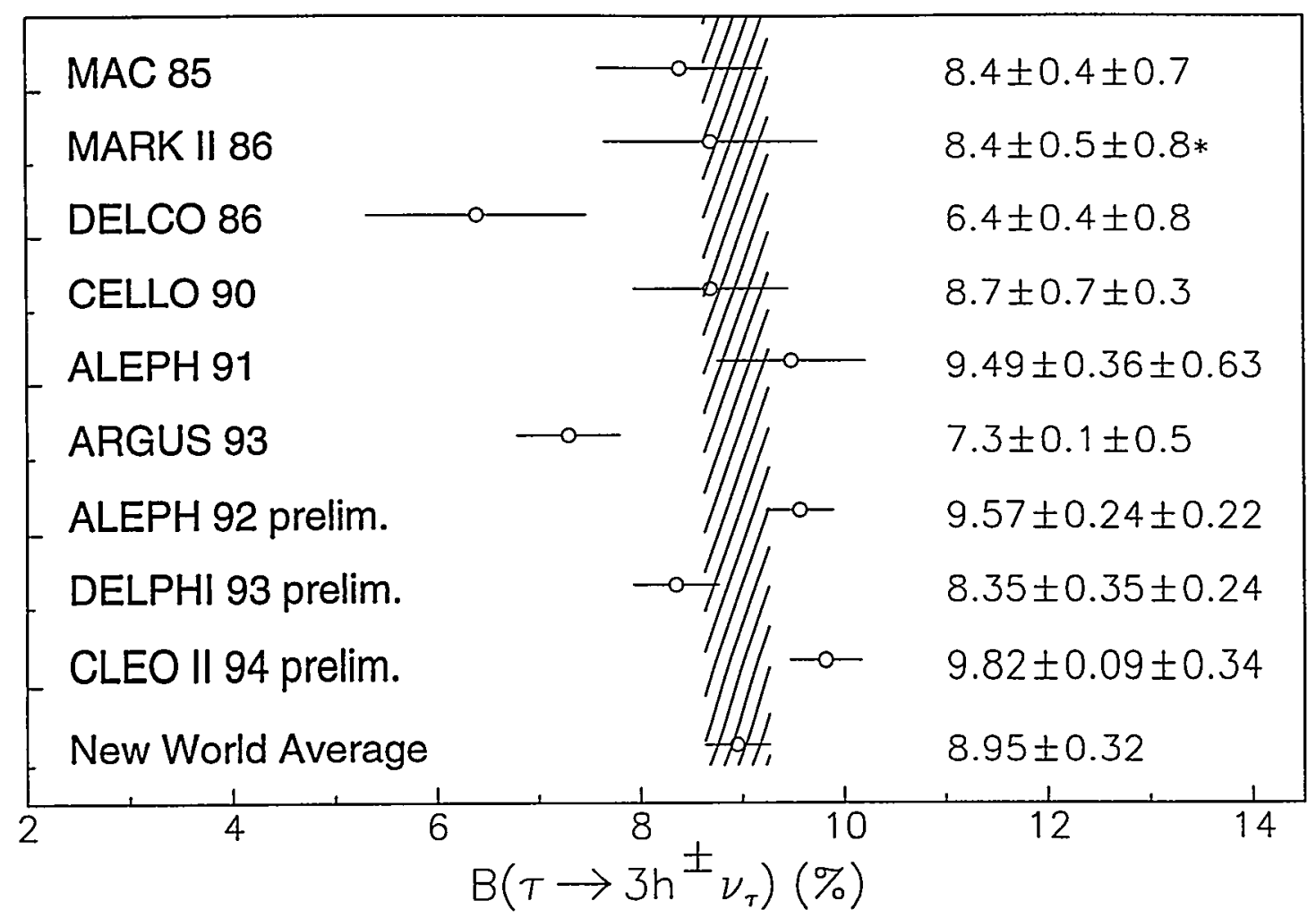

Figure 9: Published and other recent measurements of the decay mode $\tau \rightarrow 3 h^{ \pm} \nu_{\tau}$. A correction has been applied to the Mark II result.

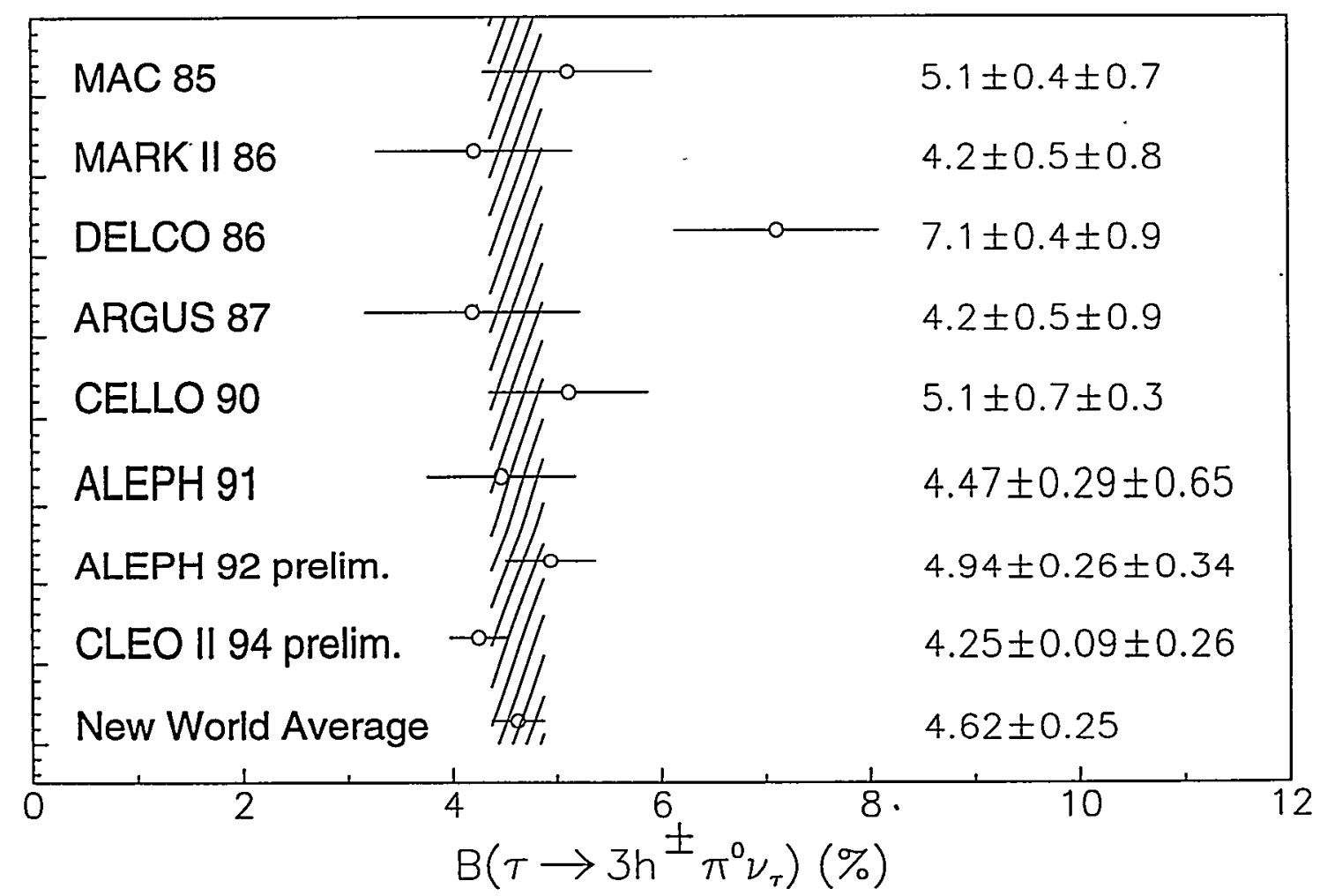

Figure 10: Published and other recent measurements of the decay mode $\tau \rightarrow 3 h^{ \pm} \pi^{\circ} \nu_{\tau}$. Correction (see text) have been applied to all but the ARGUS and CLEO results. 
and Pich obtain an expected value of $0.16 \pm 0.02 \%$ for $\tau^{-} \rightarrow K^{\circ} K^{-} \nu_{\tau}$. Decker et al. use chiral perturbation theory to predict that the $\tau^{-} \rightarrow K^{\circ} K^{-} \pi^{\circ} \nu_{\tau}$ branching ratio will be substantially smaller than those for both $\tau^{-} \rightarrow K^{-} K^{+} \pi^{-} \nu_{\tau}$ and $\tau \rightarrow K^{\circ} \bar{K}^{\circ} \pi^{-} \nu_{\tau}$ owing to a suppression of the $K^{*} K$ component in the $K^{\circ} K^{-} \pi^{\circ}$ final state. Neglecting this suppression, naive isospin predicts that if both $\tau^{-} \rightarrow K^{\circ} K^{-} \pi^{\circ} \nu_{\tau}$ and $\tau \rightarrow K^{\circ} \bar{K}^{\circ} \pi^{-} \nu_{\tau}$ proceed through $K^{*} K$, these two branching ratios should be equal; this statement remains true if the decay process is actually $\tau^{-} \rightarrow a_{1}^{-} \nu_{\tau} ; a_{1} \rightarrow K^{*} K$

Experimental measurements are also sparse because of the low decay rates of the modes in question, low detection efficiency of the $K^{\circ}$, and limited charged kaon identification capabilities of many particle detectors. The current experimental results on these decays are listed below:

$$
\begin{array}{ll}
\mathcal{B}\left(\tau^{-} \rightarrow K^{\circ} K^{-} \nu_{\tau}\right)<0.26 \% \text { at } 95 \% \mathrm{CL} & (\mathrm{TPC}) \\
\mathcal{B}\left(\tau^{-} \rightarrow K^{\circ} K^{-} \nu_{\tau}\right)=0.29 \pm 0.12 \pm 0.03 \% & (\mathrm{ALEPH}) \\
& \\
\mathcal{B}\left(\tau^{-} \rightarrow K^{\circ} K^{-} \pi^{\circ} \nu_{\tau}\right)<0.26 \% \text { at } 95 \% \mathrm{CL} & (\mathrm{TPC}) \\
\mathcal{B}\left(\tau^{-} \rightarrow K^{\circ} K^{-} \pi^{\circ} \nu_{\tau}\right)=0.05 \pm 0.05 \pm 0.01 \% & (\mathrm{ALEPH})
\end{array}
$$

The ALEPH measurement has identified $K^{\circ}$ 's based on interactions of $K_{L}^{\circ}$ 's in their calorimeter. CLEO identifies $K^{\circ}$ 's most efficiently using the decay $K_{s}^{\circ} \rightarrow \pi^{+} \pi^{-}$. For charged kaon identification, CLEO relies on ionization energy loss measurements $(d E / d x)$ in the tracking chambers. Separation of kaons from pions based on $d E / d x$ information is limited to momenta less than $700 \mathrm{MeV} / \mathrm{c}$, where the separation is better than $3 \sigma$, and greater than $2.0 \mathrm{GeV} / \mathrm{c}$, where the separation is approximately $1.6 \sigma$. For $\tau^{-} \rightarrow K_{s}^{\circ} h^{-} X$ candidate events with $p_{h^{-}}>2.0 \mathrm{GeV} / \mathrm{c}$, the $K^{-}$fraction can be determined statistically by fitting the measured $d E / d x$ distribution for the $K^{-}$candidate. We define $\sigma_{K}$ to be the deviation of the $d E / d x$ measurement from that expected for true kaons at a given momentum, in units of the known resolution. We fit the $\sigma_{K}$ distribution to two Gaussians, centered at the expected signal locations of true charged $K$ 's and $\pi$ 's. The means and widths of these $\sigma_{K}$ distributions are determined from data by using $D^{0} \rightarrow K^{-} \pi^{+}$events, where the $D^{0}$ is tagged as a $D^{*}$ daughter to reduce background. The $\sigma_{K}$ distribution for these identified $K$ 's and $\pi$ 's is shown in Fig.11.

We measure the $K_{s} K^{-}$invariant mass distribution by fitting the background-subtracted $\sigma_{K}$ distributions to two Gaussians (as described previously) in $50-\mathrm{MeV}$ bins of $K_{s} K^{-}$mass. The resulting mass distributions are shown in Fig.12. We use this distribution to determine the branching ratio for $\tau^{-} \rightarrow K^{\circ} K^{-} \nu_{\tau}$ :

$$
\mathcal{B}\left(\tau^{-} \rightarrow K^{\circ} K^{-} \nu_{\tau}\right)=(0.123 \pm 0.023 \pm 0.023) \%
$$

in good agreement with the CVC-derived predictions.

From the similar analysis of events with additional reconstructed $\pi^{0}$ we measure:

$$
\mathcal{B}\left(\tau^{-} \rightarrow K^{\circ} K^{-} \pi^{\circ} \nu_{\tau}\right)=(0.129 \pm 0.050 \pm 0.032) \%
$$

Our results represent the most precise values for these branching ratios obtained to date. Given the errors, our numbers are not inconsistent with those of the ALEPH collaboration. 
However, the indication from the ALEPH results that $\tau^{-} \rightarrow K^{\circ} K^{-} \nu_{\tau}$ is substantially larger than $\tau^{-} \rightarrow K^{\circ} K^{-} \pi^{\circ} \nu_{\tau}$ is not supported by our data. The result for $\tau^{-} \rightarrow K^{\circ} K^{-} \nu_{\tau}$ is in good agreement with the CVC-derived predictions. Preliminary results of the fits to the mass spectra for the $K^{\circ} K^{-} \pi^{\circ}$ final state are consistent with those expected from $\tau^{-} \rightarrow K^{*} K \nu_{\tau}$. The results were contributed to the Glasgow conference.

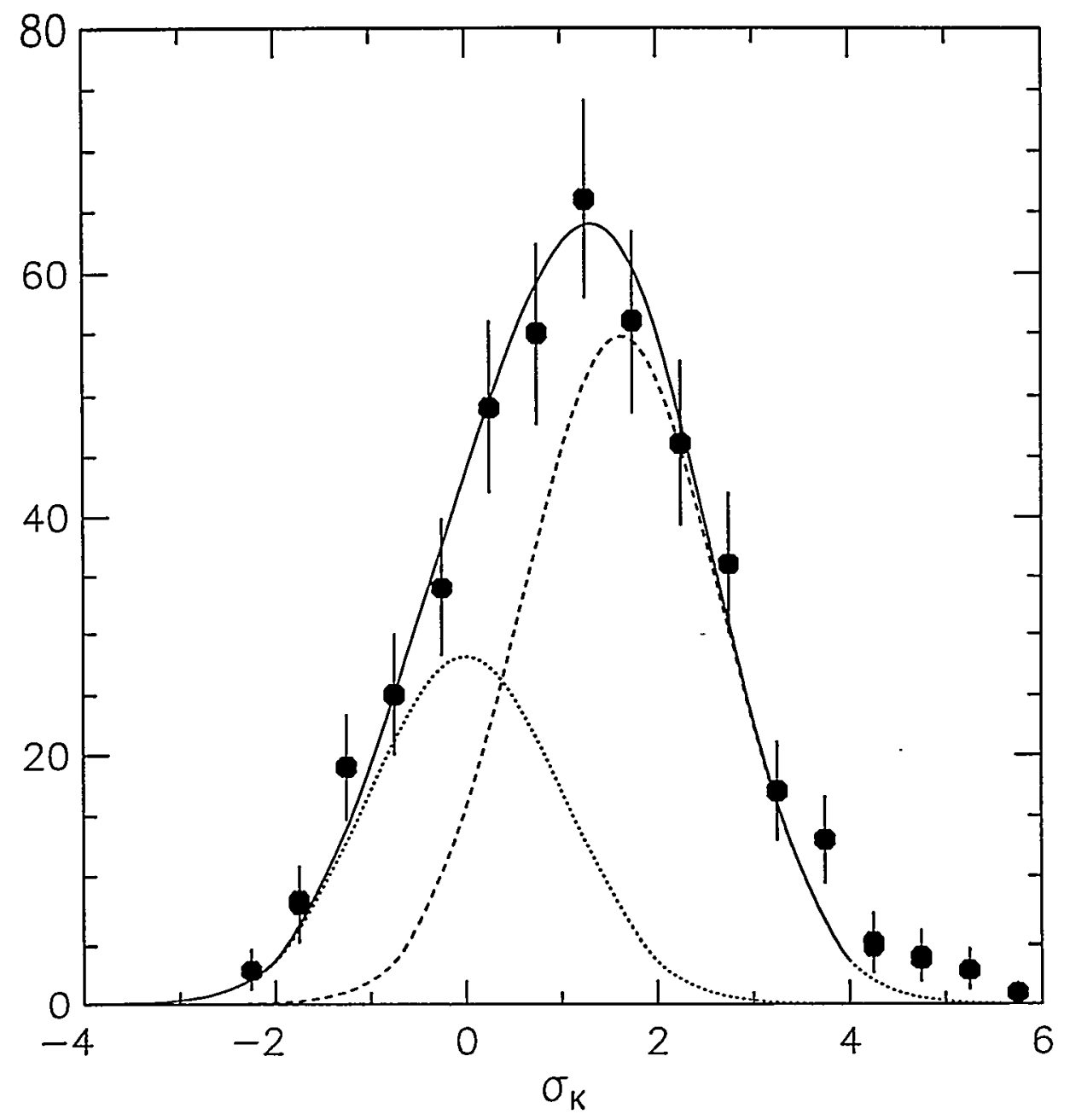

Figure 11: The $\sigma_{K}$ distributions for $K^{-}$candidates from $\tau^{-} \rightarrow K^{\circ} K^{-} \nu_{\tau}$ decays. The points are from data, the solid line shows the fit to two Gaussians, the dotted line is the Gaussian consistent with the $\mathrm{K}$ hypothesis, and the dashed line is the Gaussian consistent with the $\pi$ hypothesis. Points above 4.0 are excluded from the fit. 


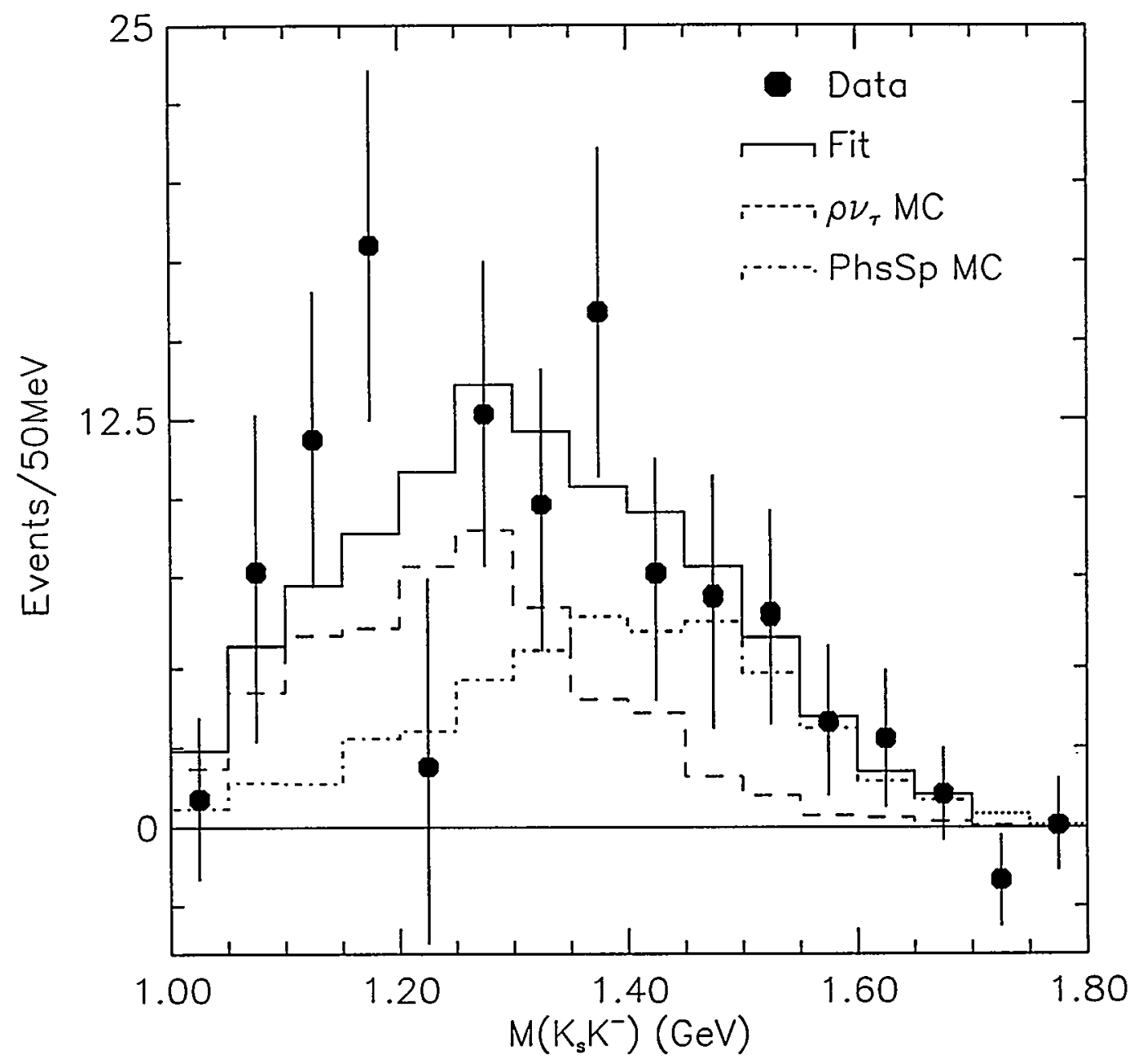

Figure 12: The $K_{s} K^{-}$invariant mass of $\tau^{-} \rightarrow K^{\circ} K^{-} \nu_{\tau}$ events. The filled circles, with error bars, are for the data. The solid histogram is the result of a fit to the $\tau^{-} \rightarrow \rho^{-} \nu_{\tau}, \rho \rightarrow K_{s} K^{-}$(dashed) and $\tau^{-} \rightarrow K^{\circ} K^{-} \nu_{\tau}$ phase space (dot-dashed) Monte Carlo models. 


\subsubsection{QCD Studies}

\section{Determination of $\alpha_{\mathrm{s}}$ from Hadronic Spectral Moments in $\tau$ Decays}

Hadronic decays of $\tau$ are one of a very few processes calculated to $\mathcal{O}\left(\alpha_{s}^{3}\right)$ in perturbative QCD and thus are expected to have a very small contribution from the missing higher order corrections. It turns out that this process also has very small hadronization effects. This has made this process indisputably the best to determine the most precise value of $\alpha_{s}$. The method involves measurements of moments of the total spectral function in the hadronic decays of $\tau$. To obtain the total spectral function invariant mass distributions of all possible final hadronic states must be measured mode-by-mode, corrected for efficiency and backgrounds, and summed together. Results from LEP have demonstrated that this is the only QCD process where experimental (largely statistical) errors are dominant over theoretical uncertainties. With the largest sample of $\tau$ data in the world, CLEO-II is well suited for improving over the first measurement of this type done by ALEPH. Sanghera has performed such analysis. He has been able to study the vector (V) and axial-vector (A) components of $\tau$ decays separately, for the first time. He has extracted also the parameters characterizing the non-perturbative dynamics of $\mathrm{QCD}$ to test predicted relations among them. This provides an insight into the poorly understood non-perturbative sector of QCD. From the above analysis he has obtained the preliminary result of:

$$
\alpha_{s}\left(M_{Z^{0}}\right)=0.114 \pm 0.003
$$

The analysis was reported at the Glasgow conference and received a lot of attention.

\section{Testing of Flavor Structure of QCD with Jet Production Rates}

Sanghera has tested the flavor structure of QCD by performing an analysis of hadronic jets measured from the CLEO data in the continuum where only four flavors are produced. All previous jet studies have been performed in five flavor continuum; and the QCD predictions of the next-to-leading order coefficients in the four flavor continuum are substantially different due to different contributions from the so-called vacuum polarization diagrams. This study constitutes a new validity test of QCD. Furthermore, it provides a unique test of renormalization group equation (used to extrapolate the results from one energy point to another), as this is the first time that the same process on both sides of the threshold has been studied to compare the results. The obtained result:

$$
\alpha_{s}\left(M_{Z^{0}}\right)=0.113 \pm 0.006
$$

is in good agreement with the high energy determinations. This analysis has also resulted in a Glasgow contributed paper. 


\section{Other QCD Tests with the CLEO - II Data}

Sanghera has initiated a multiplicity analysis of charm and light quark events in order to test a relatively new and very precise prediction of perturbative QCD that the multiplicity difference between heavy and light quark events is independent of c.m. energy. This analysis constitutes the test of the theoretical notion that QCD remains asymptotically free down to the scale $M_{c}^{2}$. The large sample of CLEO continuum data makes it possible to tag the charm events with fully reconstructed $D^{* \pm}$ and measure the most precise value of charm events multiplicity. The results are compared with those from experiments at higher c.m. energy to test the QCD prediction. The analysis is under progress.

\section{Phenomenological Papers}

Sanghera has been working on QCD phenomenology, in addition to the analysis of the CLEO-II data.

In QCD, the ambiguity in choosing the so called renormalization scale is the largest source of errors on the determination of strong coupling constant. A new experimental procedure to handle this uncertainty and to exploit the ambiguity in the scale to account for the missing higher order corrections in QCD calculations was a subject of the paper published in International Journal of Modern Physics, A. In the QCD community, there is a growing concern that $\alpha_{s}$ values determined from LEP data from different observables are inconsistent with each other if only the experimental errors are taken into account; and also that these values are consistently different than those extrapolated from the low energy experiments. The paper demonstrated that applying the proposed method to the published LEP data, both problems disappear.

A paper on the new jet-algorithms less sensitive to hadronization and hence more reliable to use in QCD studies has been submitted to Phys.Rev.D

\subsection{4 $\Upsilon$ Physics}

Physics of $b$ quark below the kinematic threshold for the production of $B \bar{B}$ mesons resembles positronium, with electromagnetic interactions replaced by the strong forces. Even though, in total 30 stable $b \bar{b}$ states are expected below the $B \bar{B}$ threshold only 9 has been observed so far. Skwarnicki searched for evidence for production, of yet undetected, triplet $\Upsilon(1 D)$ states in the radiative quadruple transitions between $\Upsilon(3 \mathrm{~S})$ and $\Upsilon(1 \mathrm{~S})$ via $\chi_{b}(2 \mathrm{P})$ and $\chi_{b}(1 \mathrm{P})$ states. One event was found and the backgrounds was shown to be negligible. This was the first observation of the $\Upsilon(1 D)$ states. These states are interesting because they are the only long-lived D-wave hadrons in nature. Measurement of their masses will provide a new insight into inter-quark potential. Publication awaits more $\Upsilon(3 S)$ data.

We have proposed together with the Kansas group to take a relatively short run on top of the $\Upsilon(2 S)$ resonance. The CLEO-II has not run on top of the $\Upsilon(2 S)$ yet. Because of 
the superb CsI calorimeter, even a short run will allow CLEO-II to significantly improve measurement of the fine structure of $\chi_{b}(1 P)$ states. This is of theoretical interest because the present data indicate that the ratio $r=\left(M_{J=2}-M_{J=1}\right) /\left(M_{J=1}-M_{J=0}\right)$ is smaller for the $2 P$ states than for the $1 P$ states in contradiction with almost all phenomenological models of spin structure of the $b \bar{b}$ interactions.

\subsection{Future of the CLEO-II program.}

The CLEO-II program undergoes an upgrade of the CESR and of the detector this year (so called "Phase-II" upgrade). Two milli-radian crossing angle at the interaction point has been introduced which will allow to store more bunches in the machine. Final focus quadrupoles will be lengthened. Masking of synchrotron radiation will be improved by installation of a new beam-pipe. Vacuum and transverse multibunch feedbacks will be improved. Improvements of luminosity be a factor of 2-3 are anticipated. At the same time inner layer of the gaseous vertex detector will be replaced by 3 layers of double sided silicon strip detector. The experiment will run in a new configuration until 1997 when another, "Phase-III" upgrade of the CESR, and a major upgrade of the detector are planned. Before the machine shuts down for the Phase-III upgrade, the CLEO-II data sample should be tripled. Improved tracking due to the presence of the silicon detector will magnify physics potential of the new sample. Until asymmetric $B$-factories at SLAC and KEK turn on at the end of the decade the CLEO-II experiment will be the only source of information on many important physics issues in the heavy flavor sector. 


\section{GEM DETECTOR AT THE SSC}

The proximity of SMU to the site of the SSC allowed the SMU group to undertake a significant role in the GEM detector. Skwarnicki coordinated the detector simulation program and Stroynowski was a lead physicist for the detector magnet and member of the GEM Executive Committee. Both of those efforts helped shape the detector design and in preparation of the Technical Design Report.

The cancellation of the SSC required an orderly closure of the ongoing activities associated with detector development. Among those, the GEM magnet commanded a lion share of the available funding. The major closeout task related to the magnet is the verification program of the novel design of the magnet conductor. This conductor, based on the "cable-in-conduit" concept, was designed to be extremely stable and to be little affected by imperfections of the winding process. Because of the potential application in other large magnet construction projects, the DOE awarded $\$ 680 \mathrm{k}$ for the completion of the verification test. A $70 \mathrm{~m}$ long piece of the conductor has been manufactured at the Y-12 plant in Oak Ridge, Tennessee and a small, $1 \mathrm{~m}$ diameter test coil has been made. This test coil has been instrumented with heaters, current sensors and control electronics. It will be tested this fall at the University of Wisconsin Magnet Test Facility where the 100,000 A power supply is available. The cryostat has been constructed and instrumented by the SSC Laboratory. The test will be run at full design current and will measure the stability of the conductor against temperature excursions, its quench propagation characteristics and other properties. The overall responsibility for the test and for the analysis of the data has been taken by the MIT Plasma Fusion Center. Extensive simulations of the coil responses to various energy depositions have been made in preparations for the test The fabrication of the conductor and its current sharing aluminum sheath has been made by welding the sheath components around the conduit with conductor. This process showed potential for damage of the conductor. The tests of other industrial processes done by the Cortaloid SA in Switzerland have shown a preference for the coextrusion technology over the welding of the components. 


\section{The CLEO-III Project}

\subsection{Overview of the CESR and CLEO upgrades}

\section{Overview of the CLEO III Detector Upgrade}

CLEO II currently probes rare $B$ decays at the level of $10^{-5}$ with integrated luminosities of $1.2 \mathrm{fm}^{-1}$ per year. It is also making high precision measurements of the properties and couplings of the $b$ and $c$ quarks and the $\tau$ lepton. An important window on new physics will be provided by increasing CLEO II's sensitivity by one to two orders of magnitude. To reach the required luminosities in a reasonable time span, an accelerator upgrade is under way to achieve instantaneous luminosities of $2 \times 10^{33} \mathrm{~cm}^{-2} \mathrm{sec}^{-1}$.

A data set of $30-50 \mathrm{fb}^{-1}$ will allow CLEO III to make precision measurements that will severely challenge the Standard Model and understand the next level of B physics including the reconstruction of exclusive $b \rightarrow u$ final states and a full analysis of all the exclusive $b \rightarrow s$ decays. In order to investigate this important physics, detector upgrades are necessary both to accommodate the requirements of the accelerator in the interaction region (IR) region and to provide the detection resolution and the particle identification (PID) needed to extract the physics. These requirements include placement of quadrupole magnets close to the interaction point. The resulting interference with the present CLEO tracking chambers demands that major components of CLEO II be replaced. These include the beryllium beam pipe, silicon detector, drift chambers and time-of-flight systems. In addition, a dedicated particle identification detector will be constructed. Both the construction of the new detectors and the modification to the IR region are scheduled to be completed by November 1997.

SMU is strongly committed to significant participation in the construction of CLEO III's dedicated particle identification detector. Indeed, it has undertaken significant $\mathrm{R} \& \mathrm{D}$ for exploring a particular technology required to implement this detector. SMU has also contributed to studies related to the new CLEO III electronics and to the machine - detector interface. Both of these general efforts will be described in detail below.

The goal of measuring rare decay processes of heavy quarks and leptons requires a very high degree of selectivity in the detector in addition to the accumulation of large data sets. The rarer the signal process the more vulnerable one is to background processes. Such backgrounds become overwhelming if the range of properties that can be measured accurately for each of the individual secondary particles is not sufficient to identify the reaction uniquely. Particle direction, charged particle momentum or neutral particle energy, and displaced vertices are all measured well by CLEO II. Particle identification $(e, \mu, \pi, K$ or $p$ ) will offer the largest gains in detector selectivity. Hence, efficient particle identification is a major design goal of CLEO III.

A list of examples of important measurements that are possible with good high momentum particle identification include: 
- Measurements of the partial widths of $\Gamma\left(B \rightarrow \pi^{+} \pi^{-}\right)$and $\Gamma\left(B \rightarrow K^{+} \pi^{-}\right)$;

- measurement of many $b \rightarrow s g$ decay modes of the form $K$ or $K^{*}$ plus $\pi, \rho, \omega$ or $\phi$ in various charge combinations;

- Comparison of the $B \rightarrow \rho \gamma$ and $B \rightarrow K^{*} \gamma$ rates and a measurement of $V_{\mathrm{ts}} / V_{\mathrm{td}}$;

- Measurement of rare charm decays, such as the double Cabibbo-suppressed $D^{0} \rightarrow$ $K^{+} \pi^{-}$

- Measurement of the $C P$ violating asymmetry in $B \rightarrow D K$;

- Measurement of $m_{\nu_{\tau}}$ via $\tau^{-} \rightarrow K^{-} K^{+} \pi^{-} \nu_{\tau}$;

- Comparisons of $\tau \rightarrow K^{-} \nu_{\tau}$ and $\pi^{-} \nu_{\tau}$ and of $\tau \rightarrow K^{*-} \nu_{\tau}$ and $\rho \nu_{\tau}$.

- Search for $2^{\text {nd }}$ class current decay $\tau \rightarrow \pi^{-} \eta \nu_{\tau}$, and related $\tau \rightarrow K^{-} \eta \nu_{\tau}$.

Overview of the CESR Luminosity Upgrade

Coincidental with the installation of the CLEO III detector will be the installation of the so-called "phase 3" equipment inside CESR to boost the machine luminosity. Replacement of the four 5-cell RF cavities with four superconducting 1-cell cavities and modification of the IR to allow more closely spaced bunches will permit increasing the beam currents to $10 \mathrm{~mA}$ per bunch. This configuration will allow CESR to reach the limits of the ring vacuum chamber (gas desorption and available pumping rate) using 9 bunch trains of 5 bunches each, with a bunch spacing around $14 \mathrm{~ns}$. The beams will cross at a horizontal crossing angle of $\pm 2.5 \mathrm{mrad}$. The proposed IR layout will accommodate values of $\beta_{V}^{*}$ down to $1 \mathrm{~cm}$.

To reach the high charge densities required for high luminosity, a very short final focal length is required, leading to placement of vertically focussing quadrupole magnets close to the interaction point. Both a superconducting quadrupole and a rare earth permanent quadrupole will be installed in a region that intrudes into the present volume of the CLEO II detector. This arrangement is shown in Fig.13. It is the placement of these magnets that will necessitate the rebuilding of all the inner trackers of CLEO II.

\subsection{Particle Identification with CLEO III}

The replacement and upgrade of CLEO II's inner tracking detectors will produce a free space of $20 \mathrm{~cm}$ radial depth at a distance of $80 \mathrm{~cm}$ from the beam line (see Fig.13). CLEO intends to fill this gap with a high quality particle identification detector capable of providing $4 \sigma$ $\pi / K$ separation up to $2.8 \mathrm{GeV} / \mathrm{c}$, the maximum possible decay momentum from a $B$ meson from $\Upsilon(4 \mathrm{~S})$ decays ( $B$ mesons are nearly at rest). 
Figure 13: Side view of one-half of the proposed CLEO-III detector.
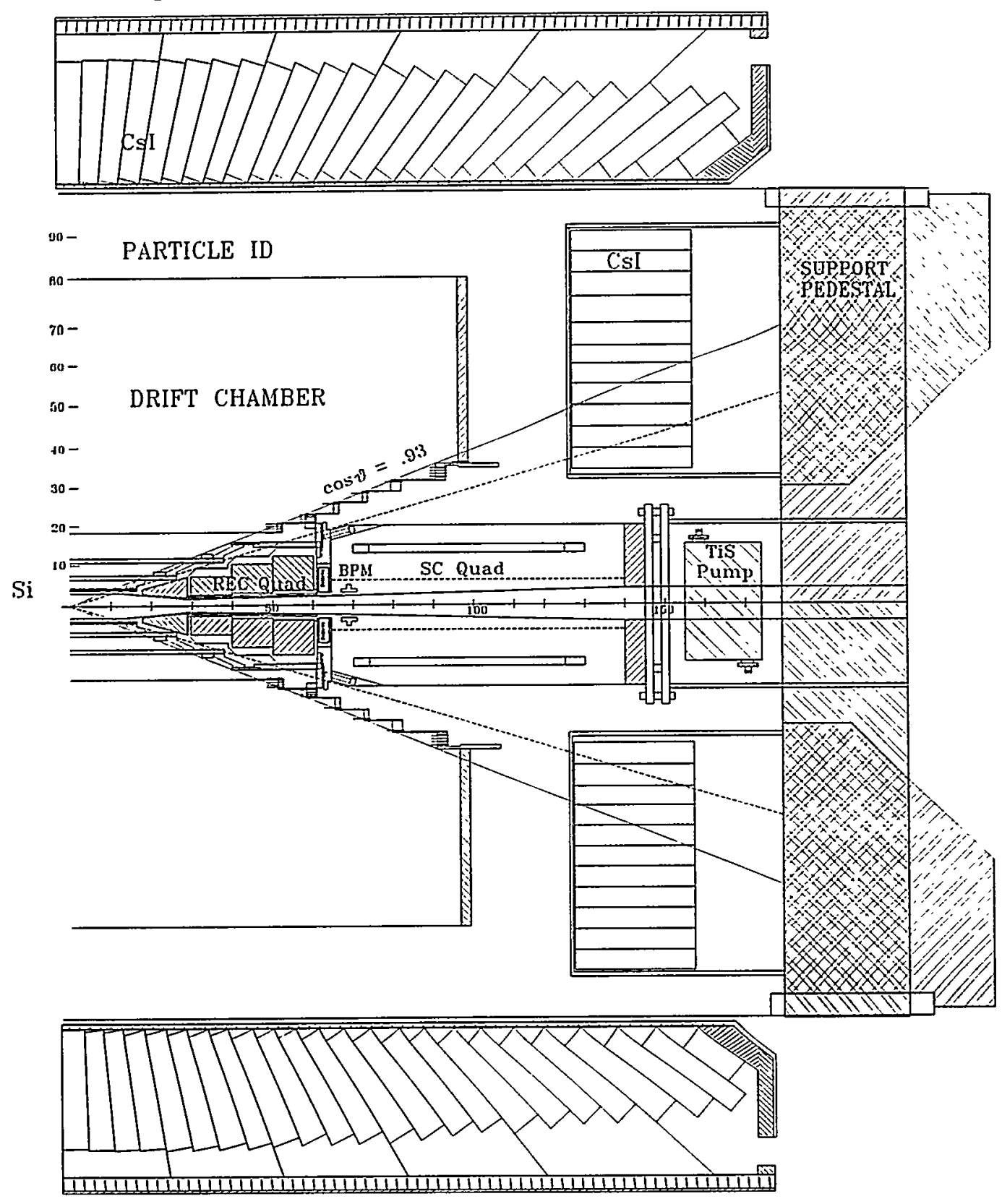


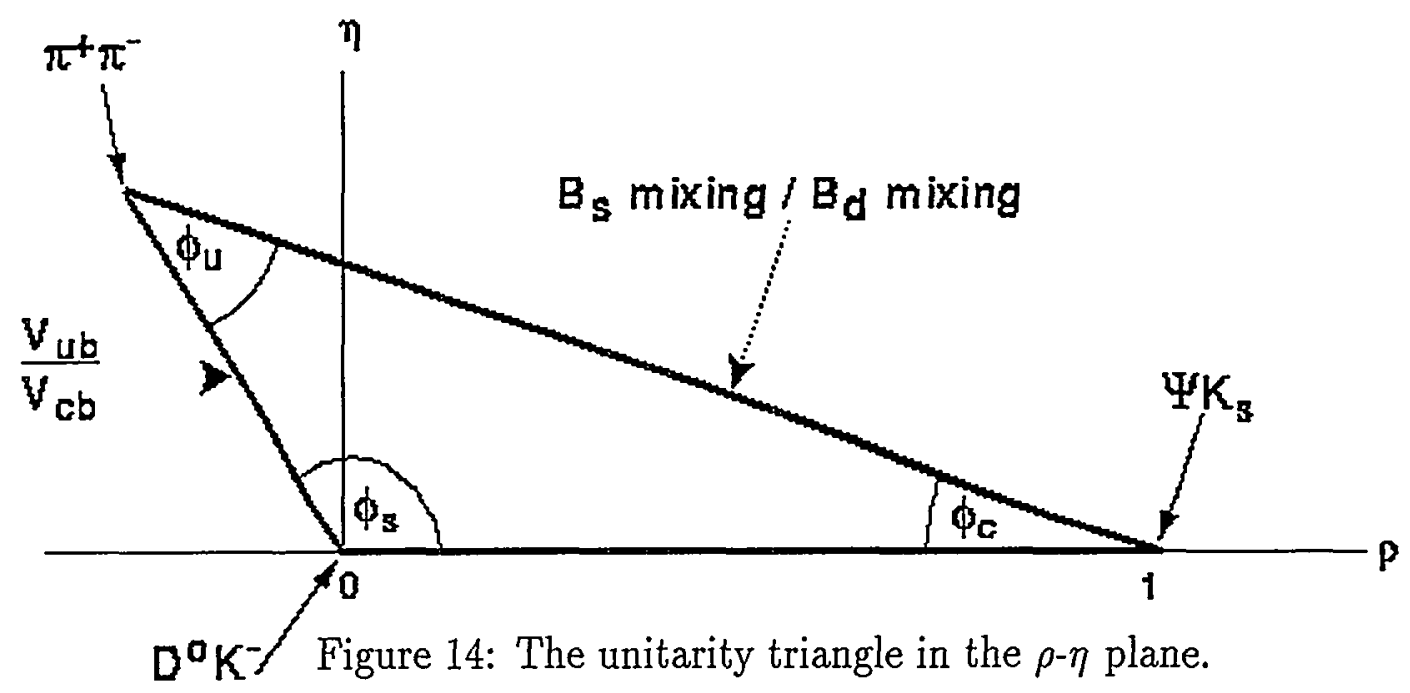

At present, CLEO relies on $d E / d x$ measurements from its main drift chamber and timing information from its time-of-flight system to provide $\pi / K$ discrimination up to about $1.0 \mathrm{GeV} / \mathrm{c}$ of particle momentum. This range allows positive identification of only $7-20 \%$ of all kaons, depending on the process involved. Extending the $\pi / K$ discrimination to higher particle momentum is a major design goal of CLEO III.

Efficient pion and kaon identification will greatly enhance CLEO's B physics reach and allow it to probe physics beyond the Standard Model. For example, measuring the ratio $\Gamma(B \rightarrow \rho(\omega) \gamma) / \Gamma\left(B \rightarrow K^{*} \gamma\right)$ in Cabibbo suppressed "penguin" decays can be used to determine the absolute value of the ratio $V_{t d} / V_{t s}$, permitting a measurement of one leg of the unitarity triangle. See Fig.14. Efficient charged hadron identification is essential to this measurement. Also, distinguishing the decay $B^{0} \rightarrow \pi^{+} \pi^{-}$from $B^{0} \rightarrow K^{+} \pi^{-}$permits a separation of $b \rightarrow u$ effects from "penguin" effects in the decay of the $B$ to light mesons. Furthermore, an attempt to observe $C P$ violation would entail distinguishing the decay $B^{0} \rightarrow K^{+} \pi^{-}$from $\bar{B}^{0} \rightarrow K^{-} \pi^{+}$. The PID system alone among all of CLEO III's detectors must provide the complete discrimination in this case.

The CLEO collaboration has pursued three competing technologies as candidates for the final technology to be used in a particle identification device:

- A silica aerogel based threshold Čerenkov counter;.

- A high-pressure, gas filled threshold Čerenkov counter;

- A ring imaging Čerenkov counter.

The first two techniques rely on the presence or absence of Čerenkov light in the detector to infer that the impinging particle was a pion or a kaon, respectively. The last technique is not a threshold technique but requires the accurate measurement of the emission angle of the Cerenkov light to infer the particle's identity. It is technically the most challenging of the three techniques. 
A major collaboration evaluation of these three technologies took place on 29-30 Aug 1994. The final detector technology choice has yet to be made although a decision on a favored technology is imminent.

SMU's R\&D effort exploring the feasibility of the first technique will be discussed in detail below.

The high pressure tube threshold Čerenkov counter is a double layer bundle of gas filled tubes arranged in a close-packed array. The $2.2 \mathrm{~m}$ long tubes are parallel to the beam line and are filled with $\mathrm{SF}_{6}$ at $20 \mathrm{~atm}$ pressure so that the refractive index $n$ of the gas is $n=1.02$. The interior of the tube has a a saw-toothed mirror at its mid-point and a reflective lining to redirect the Cerenkov light to both ends of the tube where a photon transducer is placed. A schematic of the detector is shown in Fig.15. A test tube has been built and is currently being exposed to cosmic ray muons to measure the light yield as a function of incident particle angle. In addition, detailed measurements of the transmissivity of the $\mathrm{SF}_{6}$ are in progress. A laser system has also been built to study the transmission efficiency of the overall optical system. Physicists from 5 universities in the CLEO collaboration are exploring this detector technology.

A proximity focused ring imaging Cerenkov detector (RICH) is the technically most ambitious of the three particle identification technologies for providing $\pi / K$ discrimination. It relies on a precise measurement of the emission angle of the Cerenkov ring to infer the incident particle identity. The CLEO III proposal comprises (1) a $1 \mathrm{~cm}$ thick solid LiF radiator, (2) an expansion gap of $15 \mathrm{~cm}$ filled with a UV transparent gas, (3) a CaFl window separating the expansion gap from a $1 \mathrm{~mm}$ thick multiwire chamber with cathode pad readout using TEA as a photon converter in $\mathrm{CH}_{4}$. prototype has been built with collaborators at the Collège de France and installed in a hadron beam at CERN. Figure 16. shows the CERN system for testing RICH counters.

SMU has pursued aggressively the development of a threshold Čerenkov detector with commercially available silica aerogel as the radiating element. Such a threshold detector is extremely attractive because of its inherent simplicity. Aerogel is inert, has a long radiation length, can be cast in arbitrary shapes and over a wide range of refractive indices, is easily machinable and is transparent to visible light. Simple, non-imaging optics can be used to collect its light.

We have constructed a test cell with 4 slabs of $1.7 \mathrm{~cm}$ thick aerogel produced by Swedish Airglass Co. with refractive index $n=1.02$. Such a refractive index is appropriate for $\pi / K$ discrimination over the momentum range $\sim 700 \mathrm{GeV} / \mathrm{c}-2.8 \mathrm{GeV} / \mathrm{c}$. This cell has been constructed with appropriate focusing optics and readout by a Philips XP2020 photomultiplier tube. The data acquisition system was implemented using Labview software running on a Sun Sparcstation. See Fig.17. The current focusing system is simple and incorporates both specular reflectors (aluminized mylar) and diffuse reflectors (Millipore paper). The XP2020 was selected because of its single photon resolution.

Using cosmic ray muons we have measured an average light yields of 8 photoelectrons per muon. See Fig.18. This light yield permits, in principle, a $\pi / K$ separation of $\sim \mathcal{O}\left(10^{-4}\right)$ at a momentum of $2.8 \mathrm{GeV} / \mathrm{c}$. Further improvement can be expected by optimizing the simple focusing optics and carefully selecting a photon transducer with a photocathode response that better matches the effective transmissivity of the aerogel. For example, Fig.19 shows 
Figure 15: Schematic view of the conceptual design for the proposed high pressure tube threshold Cerenkov PID detector Schematic of a single HPT element
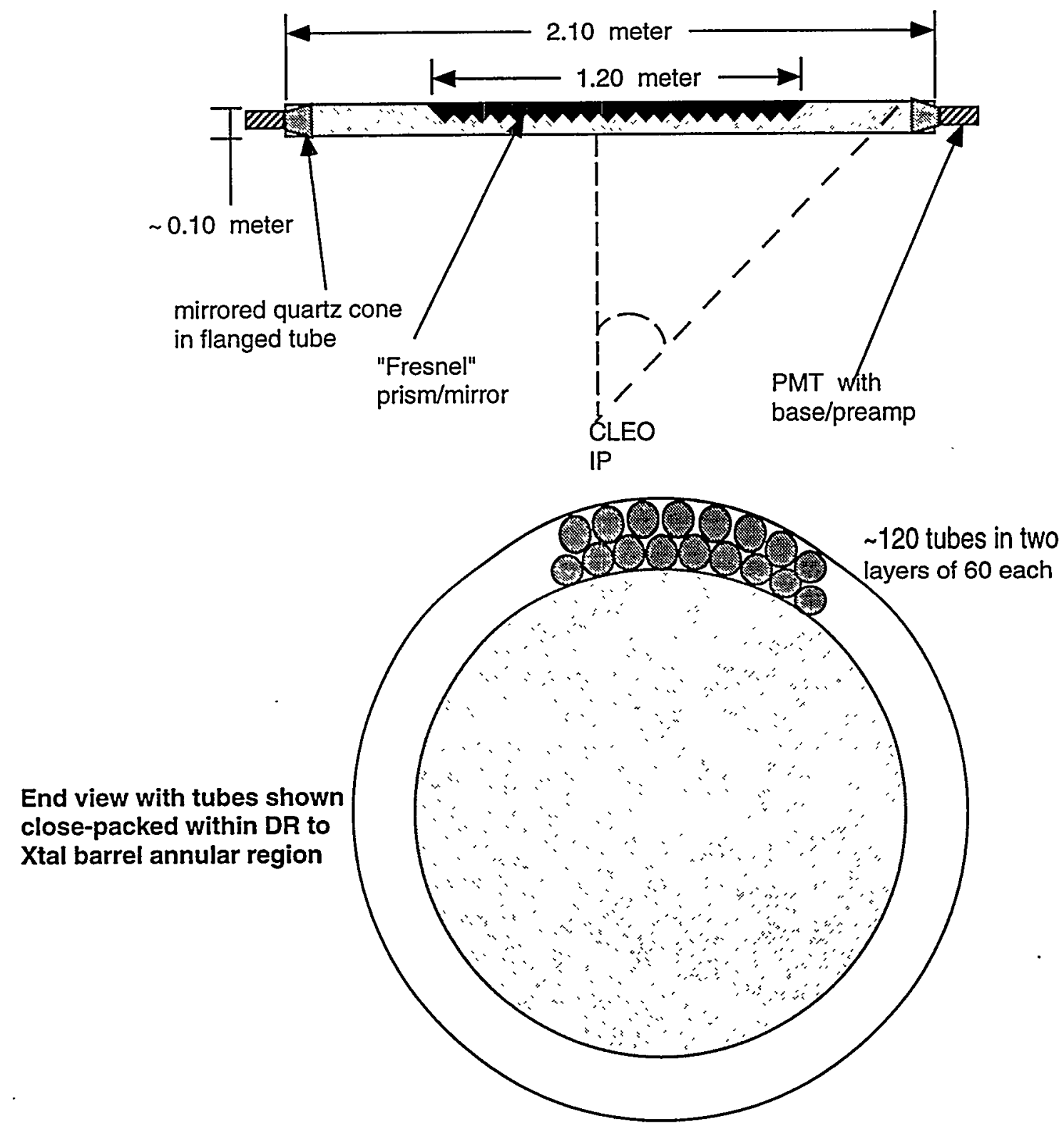
Figure 16: Axial view of the Fast RICH test setup at CERN.

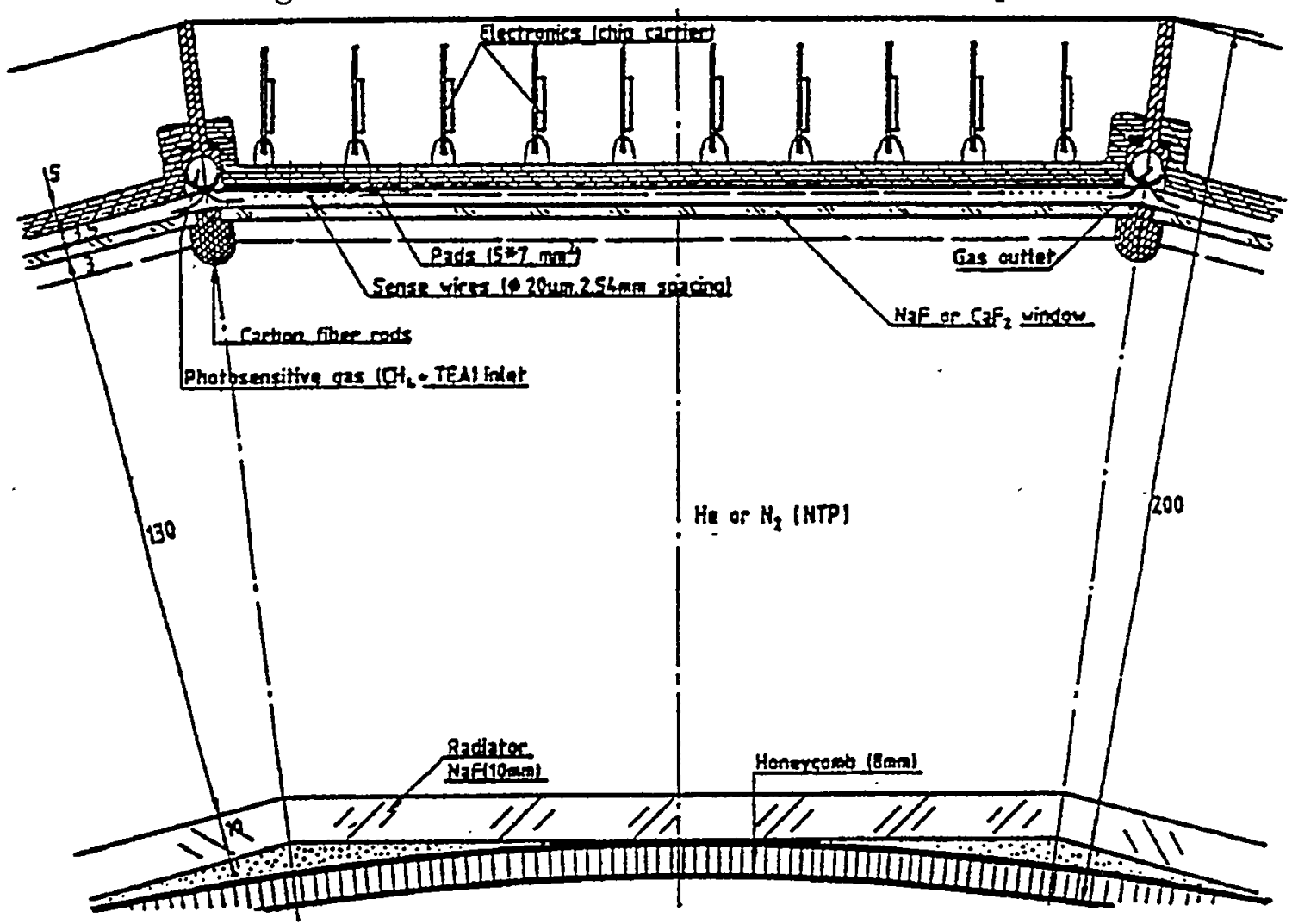


Figure 17: Aerogel cosmic ray test cell at SMU.

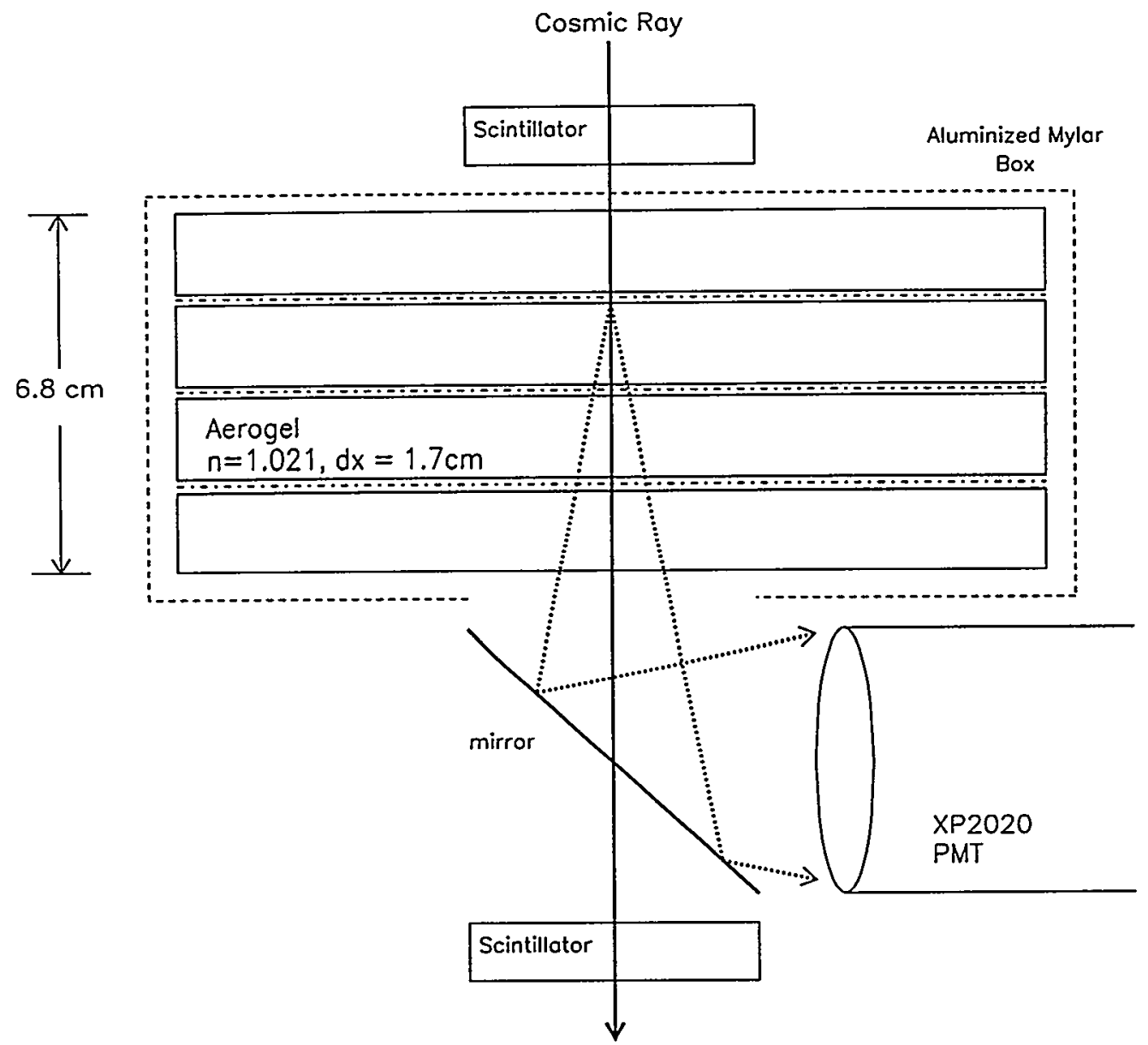


Figure 18: Photoelectron distribution measured with cosmic rays and the SMU test cell.

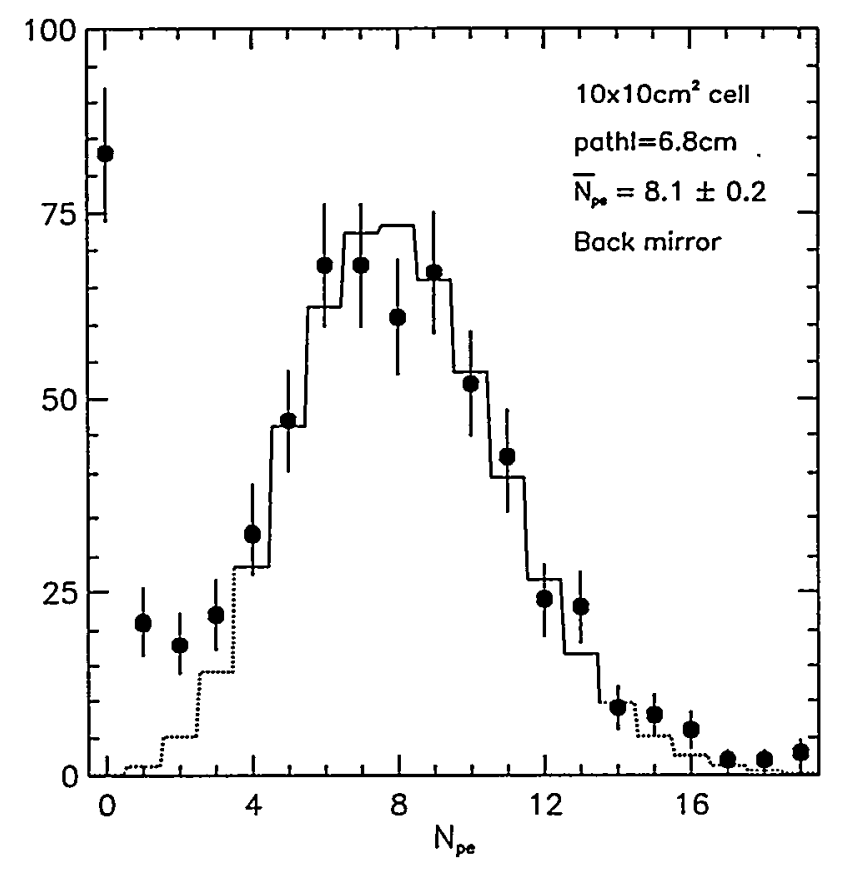

the relative light yield of an Intevac Corp. multichannel plate incorporating a high quantum efficiency photocathode (solid line) and pmt with a bialkali response (dotted line). Both curves include the effects of folding in the aerogel transmissivity and the photocathode response with the Čerenkov emission spectrum. The relative advantage of using a readout device with the appropriate photocathode response is clear.

Based on our aerogel test cell results, we have conceptually designed a full scale detector with $82 \%$ of $4 \pi$ coverage and a radiation thickness of less than $8 \%$. This barrel shaped detector has 1080 non-projective unit cells arrayed in 20 separate rings along the beam line. Fig.21 is an $r-z$ view of one $z$ strip of this detector. A detailed view of a unit cell is shown in Fig.20. Such a detector is easy to construct, mechanically robust and straightforward to read out once the final choice for the photon transducer has been made. The installation of the detector inside CLEO III would be relatively simple.

SMU currently owns a $2 \mathrm{~T}$ electromagnetic with a $15 \mathrm{~cm}$ diameter pole face and a $10 \mathrm{~cm}$ pole gap. This magnet is well matched to the $1.5 \mathrm{~T}$ CLEO magnetic field and is a convenient 
Figure 19: Relative light yield versus wavelength for the two final detector candidate readout devices, an Intevac Corp. multi-channel plate (MCP) and a Hamamatsu R5504 photomultipier tube (PMT). The effects of the Čerenkov photon production spectrum, aerogel transmissivity and photocathode response have all been included.

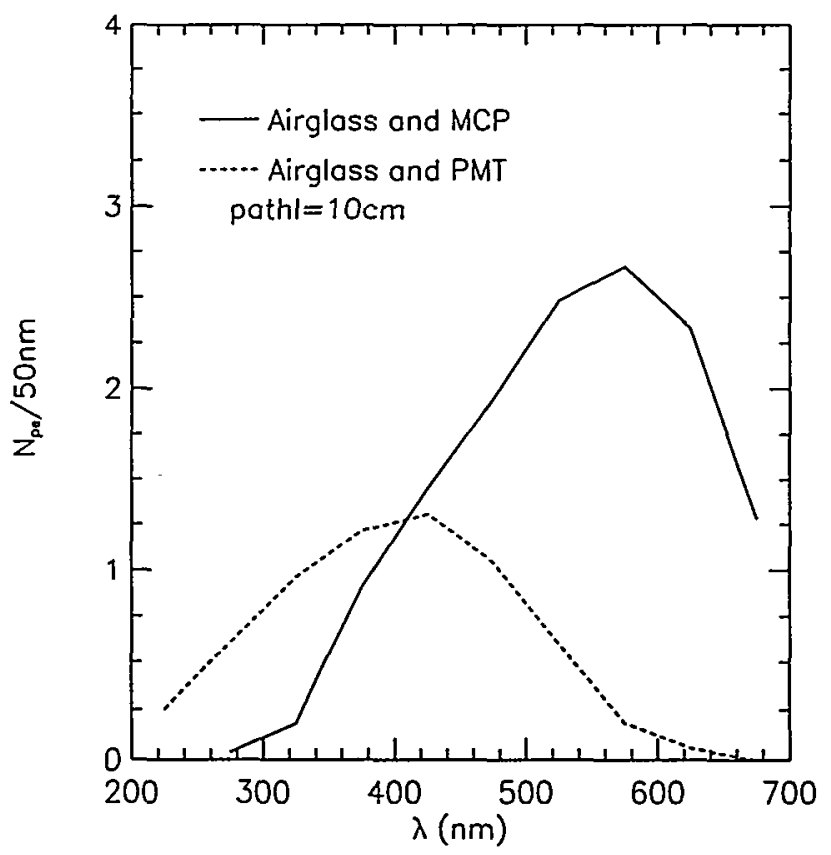


Figure 20: Conceptual design of the unit cell for the aerogel-based threshold Cerenkov PID detector.

\section{Focusing Cell \\ (tested with XP2020 PMT)}
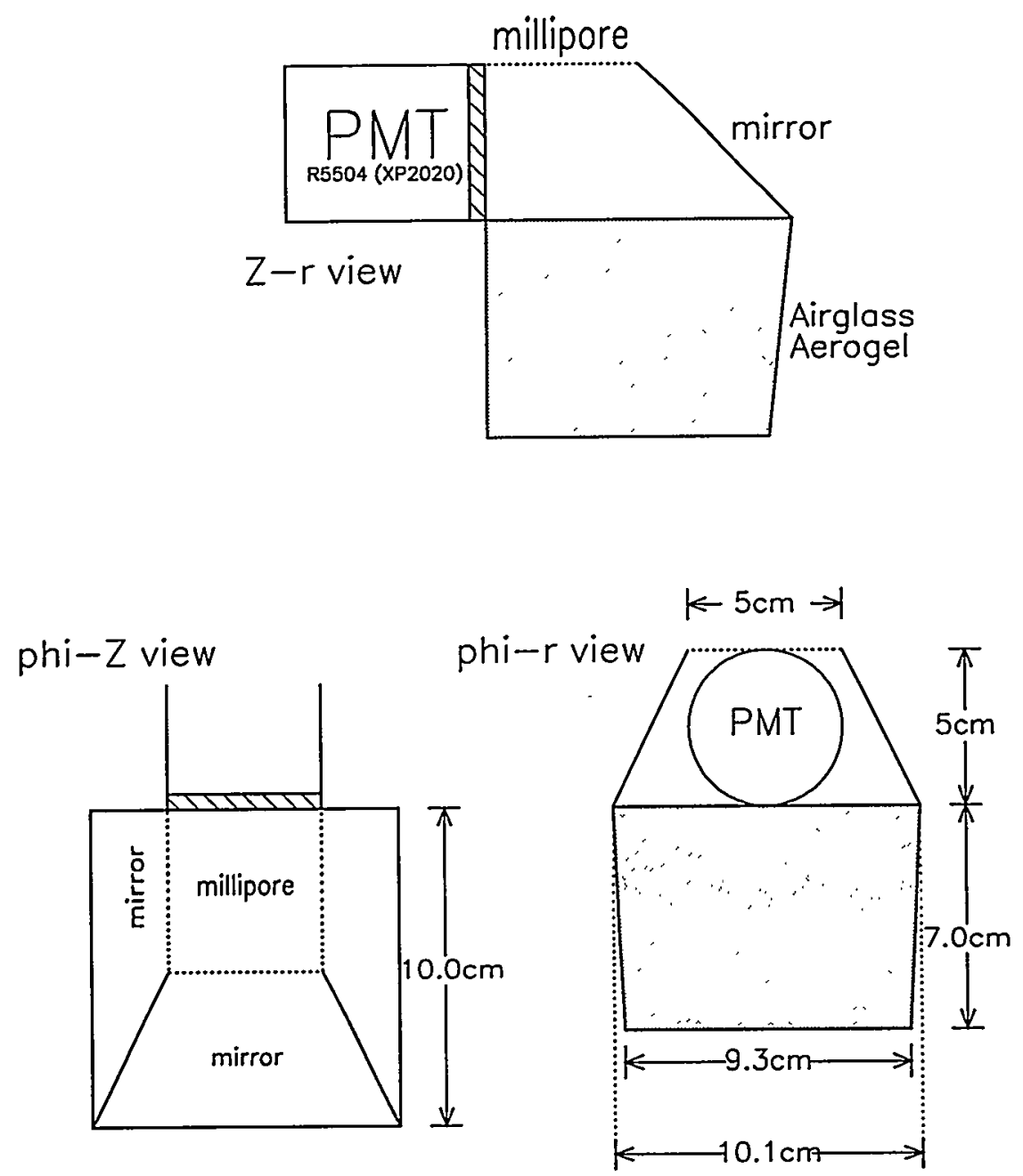
Figure 21: A side view of one $z$ strip of unit cells for the aerogel-based threshold Cerenkov PID detector.

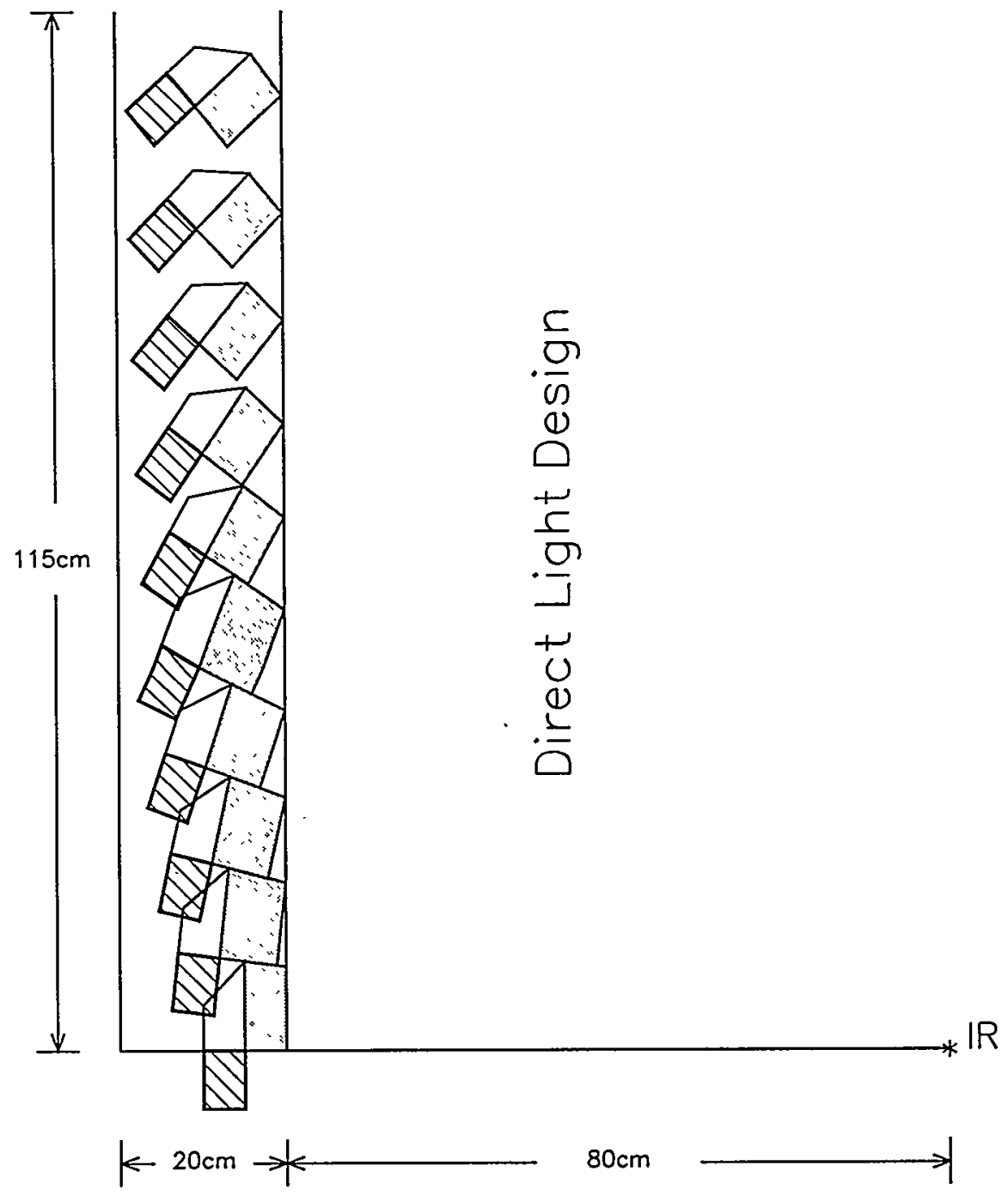


test facility for establishing the magnetic field compatability of readout devices and electronic components intended for CLEO III.

A preliminary involvement has recently started with the other threshold Cerenkov detector option, the high pressure tubes. The requirement of a high internal pressure coupled with the requirements that the detector have good solid angle coverage and be a minimum of radiation lengths thick places severe constraints on the detector's mechanical configuration. SMU has started mechanical designs for the end flange of the tube bundle, the most challenging of the mechanical tasks for this detector. In Fig.22 is shown an $r-\phi$ view of a portion of the end flange. The individual tubes are positioned inside 2 sprockets that maintain the close-packing configuration. The sprockets are joined by detachable spokes that provide the necessary mechanical strength and permit clearance for cabling and serial gas flow plumbing. The detachable spokes permit in situ replacement of a defective tube. Calculations show that aluminum alloy $7075-\mathrm{T} 6$ of $0.5 \mathrm{~cm}$ thickness can be used for both sprockets and spokes to provide sufficient mechanical rigidity and strength.

In addition, SMU has also started the mechanical design of the individual tubes, including tube material and manufacturing technique. Calculations indicate that either carbon fiber or polyamide plastic can be used to construct a tube of sufficient strength and rigidity and with a tube wall thickness of less than $1 \%$ of a radiation length.

SMU will maintain this involvement in the high pressure tube option until CLEO makes a final decision on the preferred technology choice for PID.

\subsection{Other CLEO III Projects}

In addition to the $R \& D$ work on particle identification technology, SMU has undertaken some additional studies related to the interface of the machine and the detector and to the upgrade of the electronics for CLEO III. This SMU work was carried out at CESR exclusively by SMU graduate students in collaboration with senior CLEO physicists.

\section{Beam Pipe Cooling Tests for CLEO III}

The increased luminosity during CLEO III operation will substantially increase the heat load on the beam pipe at CESR's interaction point (IP). Estimates indicate that the total heat load from beam induced eddy currents could range from $30 \mathrm{~W}-400 \mathrm{~W}$, depending on the details of the shape of the beam profile. Since CLEO's silicon microstrip detector is directly mounted onto the beam pipe at the IP, this heat must be exhausted efficiently to prevent thermal distortions to this precise tracking device.

The anticipated design of the beam pipe at the IP is a pair of coaxial tubes of elliptical cross-section. The $0.5 \mathrm{~mm}$ gap between the tubes will be filled with a coolant, either a gas or liquid. The design goal is to limit the temperature rise $\Delta T$ of the outer tube during data taking to $\Delta T<3^{\circ} \mathrm{C}$.

To select coolant type and a flow rate, SMU graduate student Vitalii Fadeyev, with collaborators, designed, built and tested a test system comprising a short sample of an IP 
Figure 22: An $r-\phi$ view of a portion of the end flange of the high pressure tube threshold Cerenkov detector. This conceptual design preserves the close-packing of the individual tubes and allows in situ replacement of a defective tube.

\section{SPROCKETS WITH SPOKES INSTALLED \\ R-PHI VIEW}

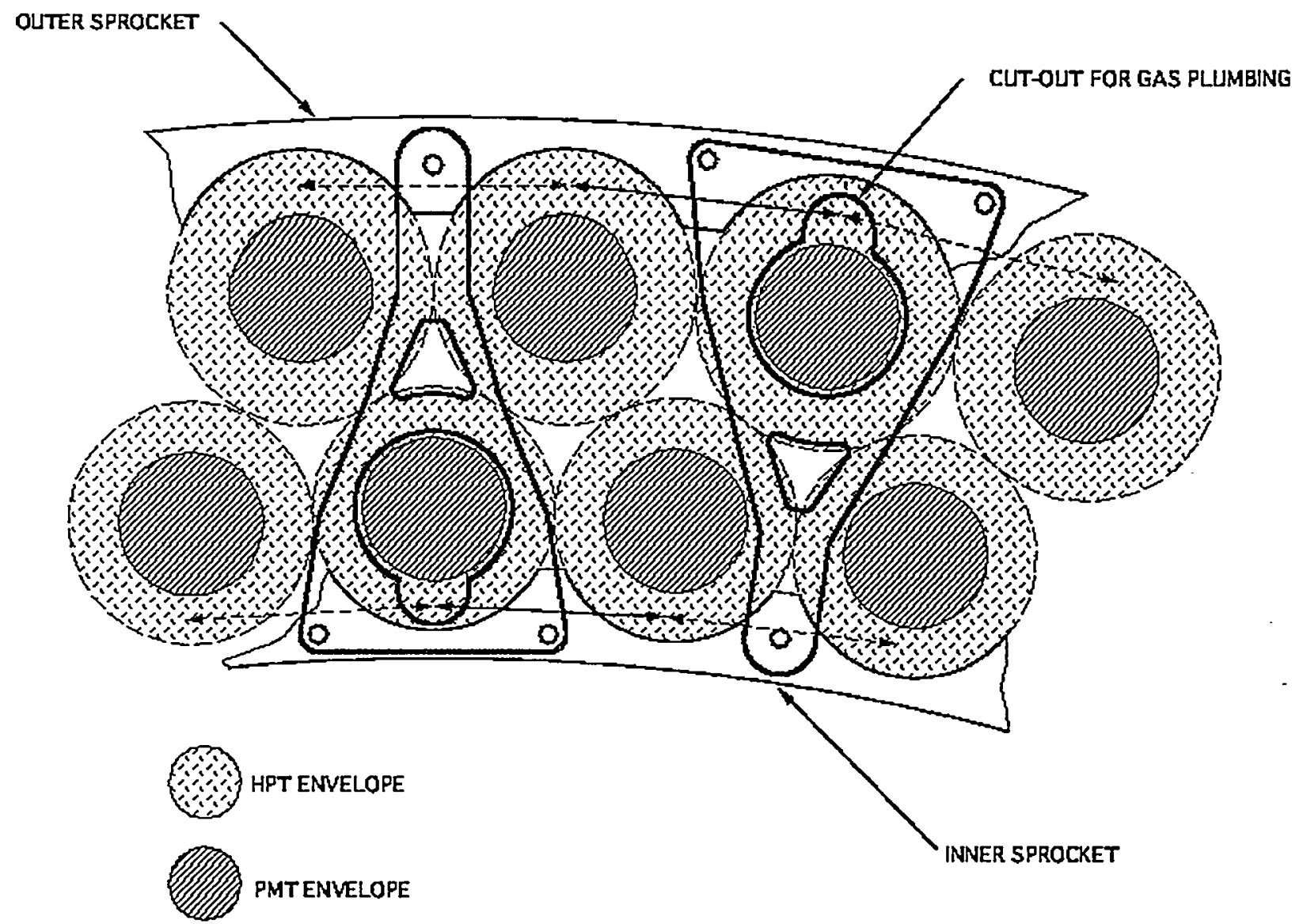

13 AUIG 94 TE. COAN 
coaxial beam pipe, heating elements attached to the sample beam pipe to simulate the heat load, and thermistors to measure the temperature rise of the coolant. The test system is is appropriately insulated to define conduction paths. A schematic figure of the test system is shown in Fig.23 for the case when the coolant is air. Fig.24 indicates the geometry of the sample section of the aluminum beam pipe.

The measured coolant temperature rise a s function of applied heat load is shown in Fig.25 for the case when the coolant is air. By comparison, the predicted temperature rise from a model of the system is shown for an air flow of 150 standard cubic feet per hour (scfh). Although the model overestimates the temperature rise for a given heat load, the linearity between the temperature rise and the applied heat load is good, as expected for the relatively simple geometry of the beam pipe. The large temperature rise indicates that these coolant flow rates are too small for efficient beam pipe cooling. As a potential improvement, Fadeyev has selected and installed a larger pump and the associated filters and plumbing to make the $\Delta T$ measurements at a larger flow rate. These measurements are in progress.

\section{Final Focus Corrector Magnet}

The increased beam currents required to achieve the high instantaneous luminosity of CESR phase three operation will require that additional corrector magnets be installed inside CESR. The new beam orbits and crossing angle demand that the beam pipe shape at various locations around CESR be changed. These new beam pipe shapes in turn require that some existing corrector magnets be replaced by new ones with the appropriate magnetic field properties.

SMU graduate student Ilya Korolkov, with collaborators, has designed three new Panofsky skew magnets for CESR. These are corrector magnets with a fixed wire wrapping geometry that can either bend or focus the beam depending on the sense of the current flow through the windings.

Using the POISSON magnet design program, Korolkov has calculated the iron geometry and field wire geometry for the three prototypes. Fig. 26 is a cross-sectional view of a typical Panofsky skew magnet showing the two sets of windings. The predicted magnetic lines of force when the constructed prototype is run as a dipole are shown in Fig.27. For the dipole mode, the design goal is to minimize the spatial non-uniformity of the field inside the iron. The prototype has a measured fractional non-uniformity over its interior of less than $3 \%$. The predicted field lines of the same prototype when run as a quadrupole are shown in Fig.28. The design goal in this case is to minimize the field gradient inside the iron along the magnet axis.

Korolkov also participated in the construction of the device used to measure the magnetic field as a function of position for the prototype. The field measuring apparatus is shown in Fig.30. The magnet under test has its current applied as a step function with a width of 3 seconds and with an overall period of 10 seconds. The magnetic flux is then intercepted by a wire loop of precise area and the resulting induced emf is then measured to infer the value of the applied magnetic field at some position. The measuring loop is then moved by 
Figure 23: Test setup used to evaluate the cooling efficiency of various cooling schemes for the CLEO III test prototype beam pipe.

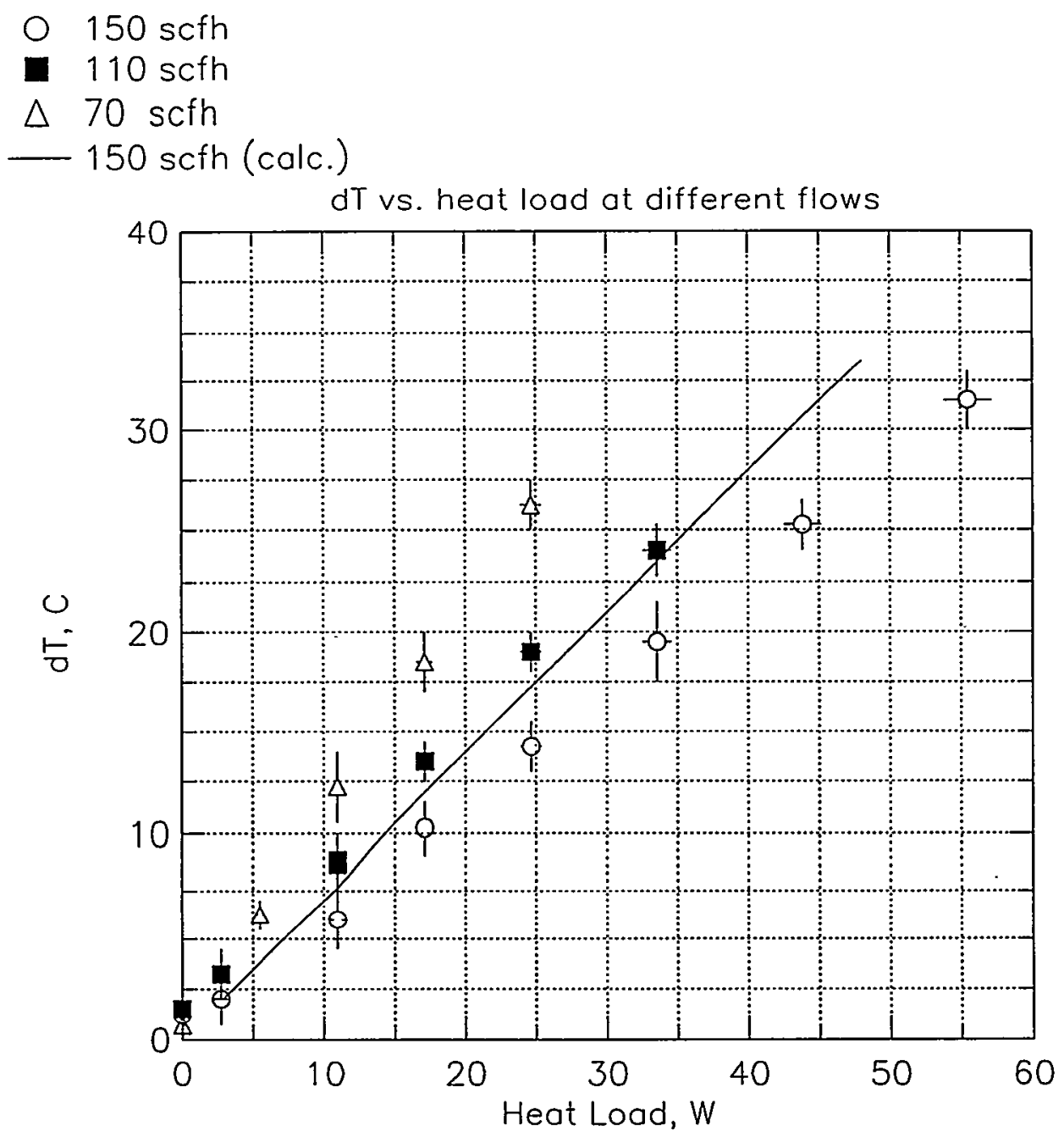


Figure 24: Sample section of the CLEO III test prototype aluminum beam pipe.

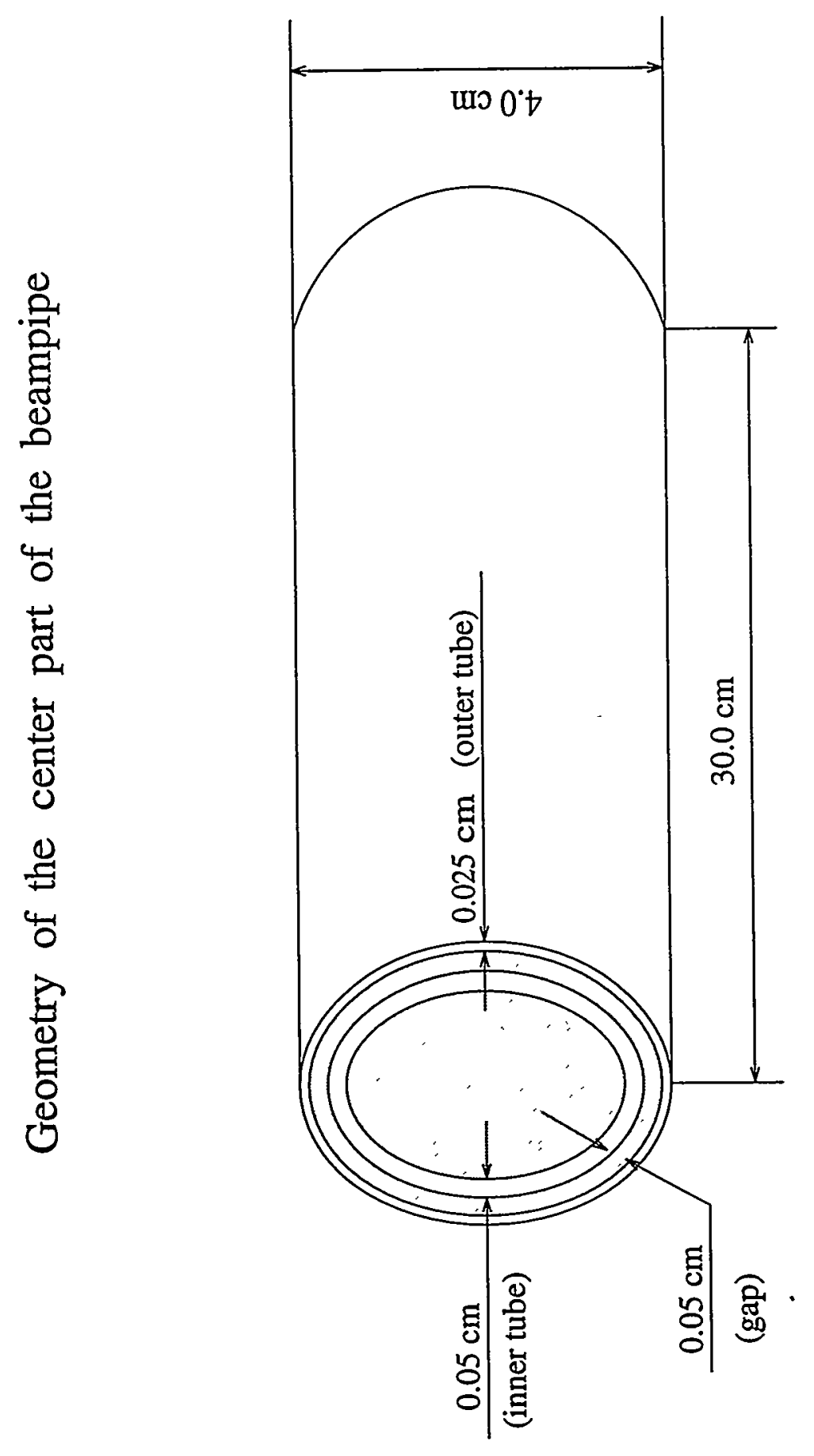


Figure 25: Temperature rise as a function of applied heat load for the CLEO III test beam pipe. The temperature rise is measured with respect to the ambient temperature.

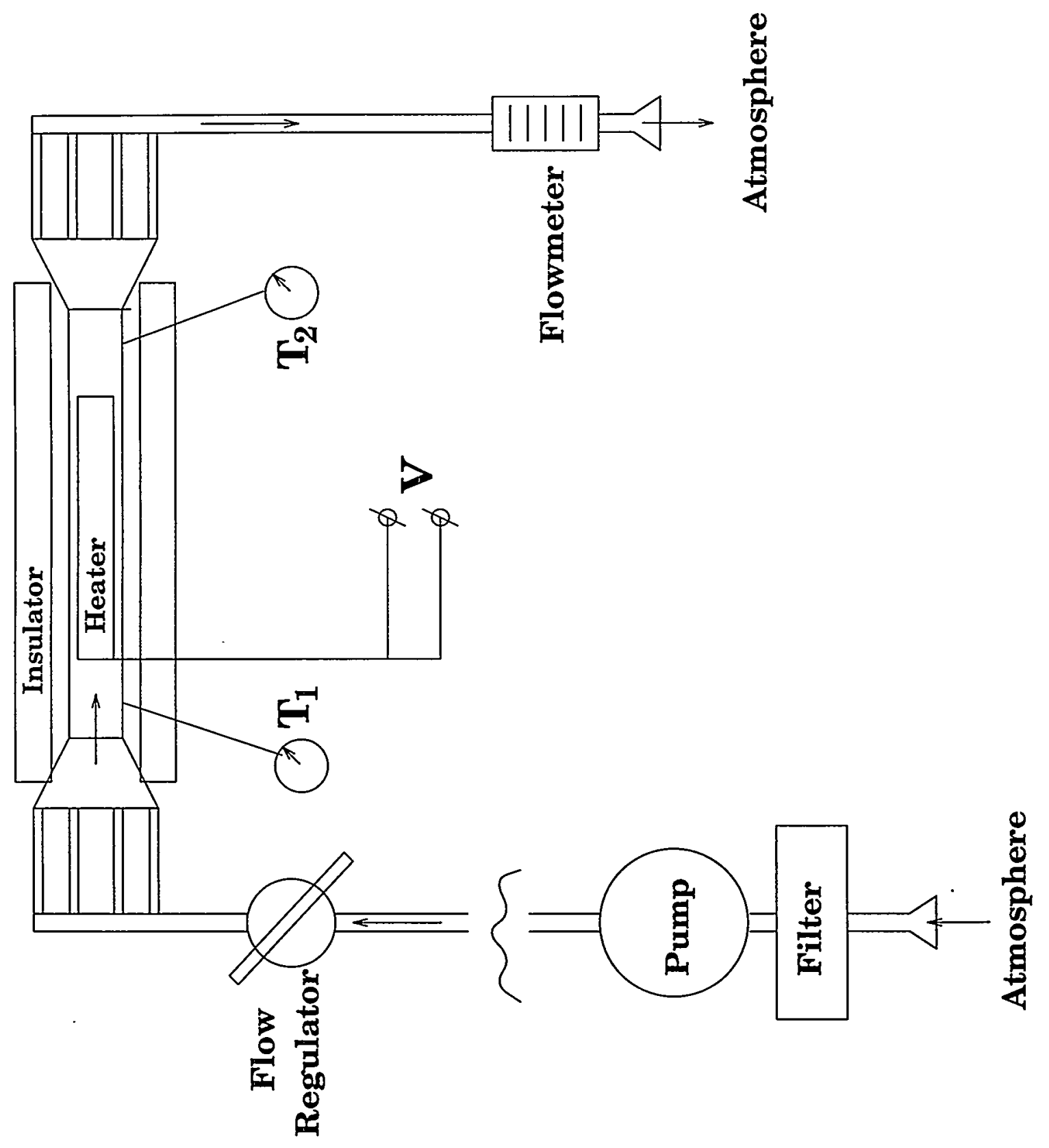


Figure 26: A cross sectional view of a portion of the CLEO III prototype Panofsky skew magnet.

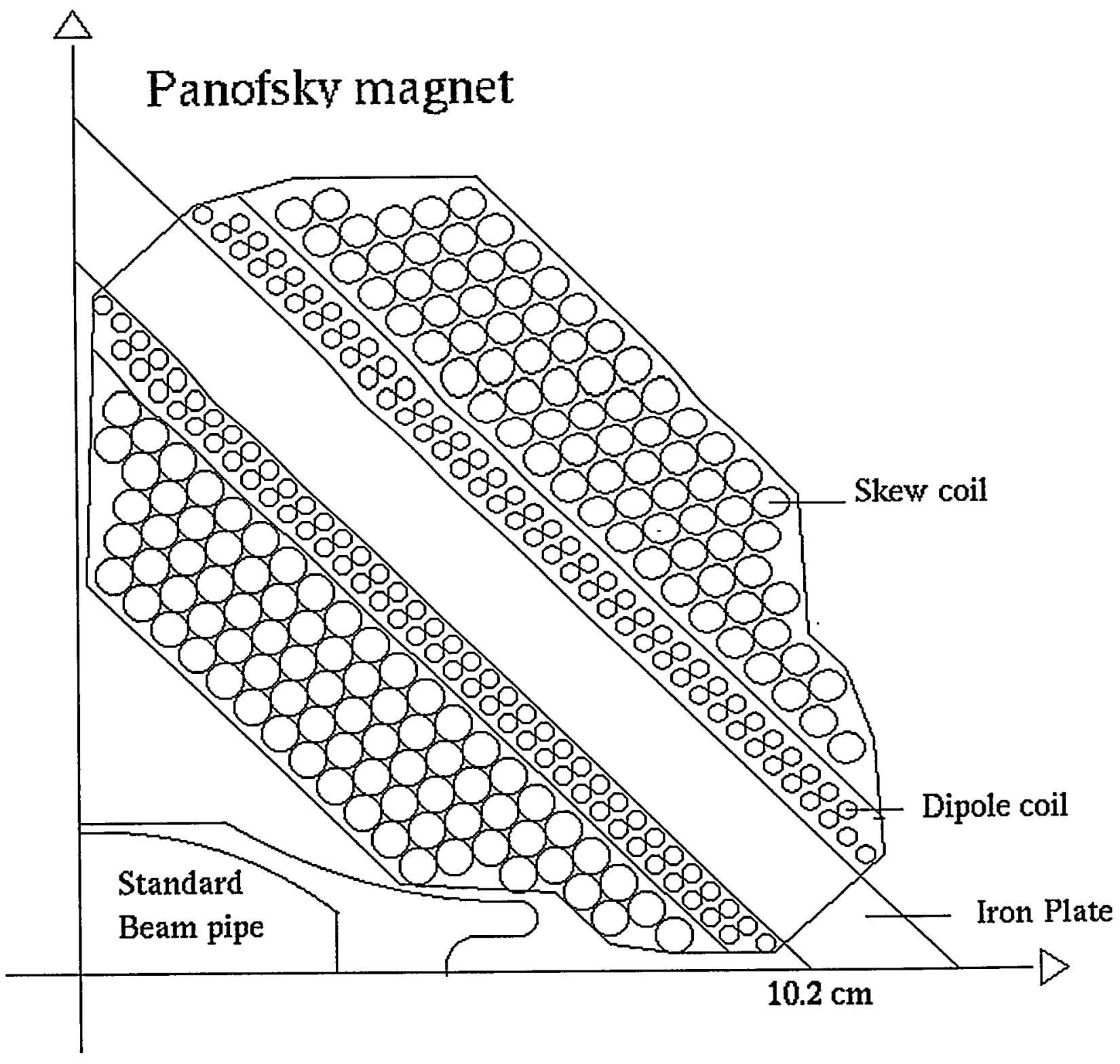


Figure 27: A cross sectional view of one-half of the prototype Panofsky skew magnet showing the magnetic lines of force when the magnet is run in dipole mode.

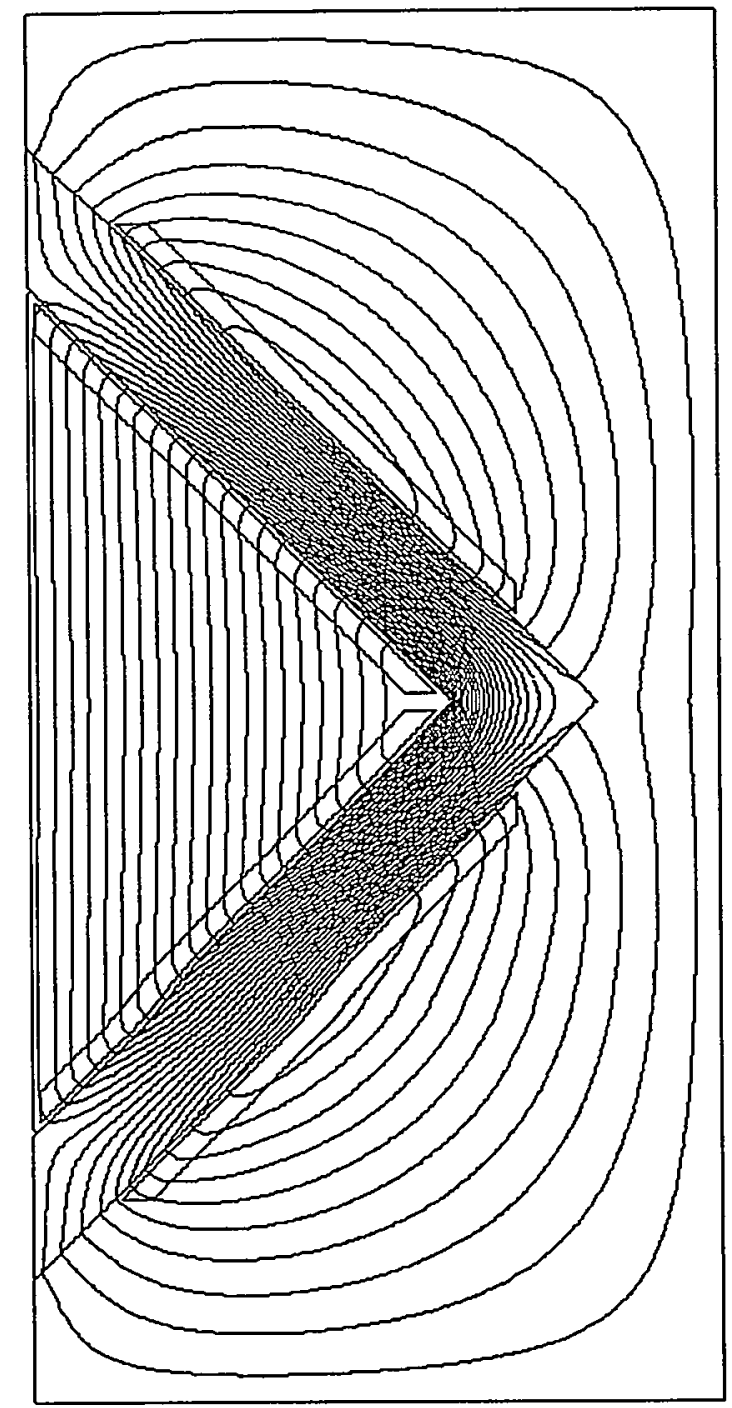


Figure 28: A cross sectional view of one-half of the prototype Panofsky skew magnet showing the magnetic lines of force when the magnet is run in quadrupole mode.

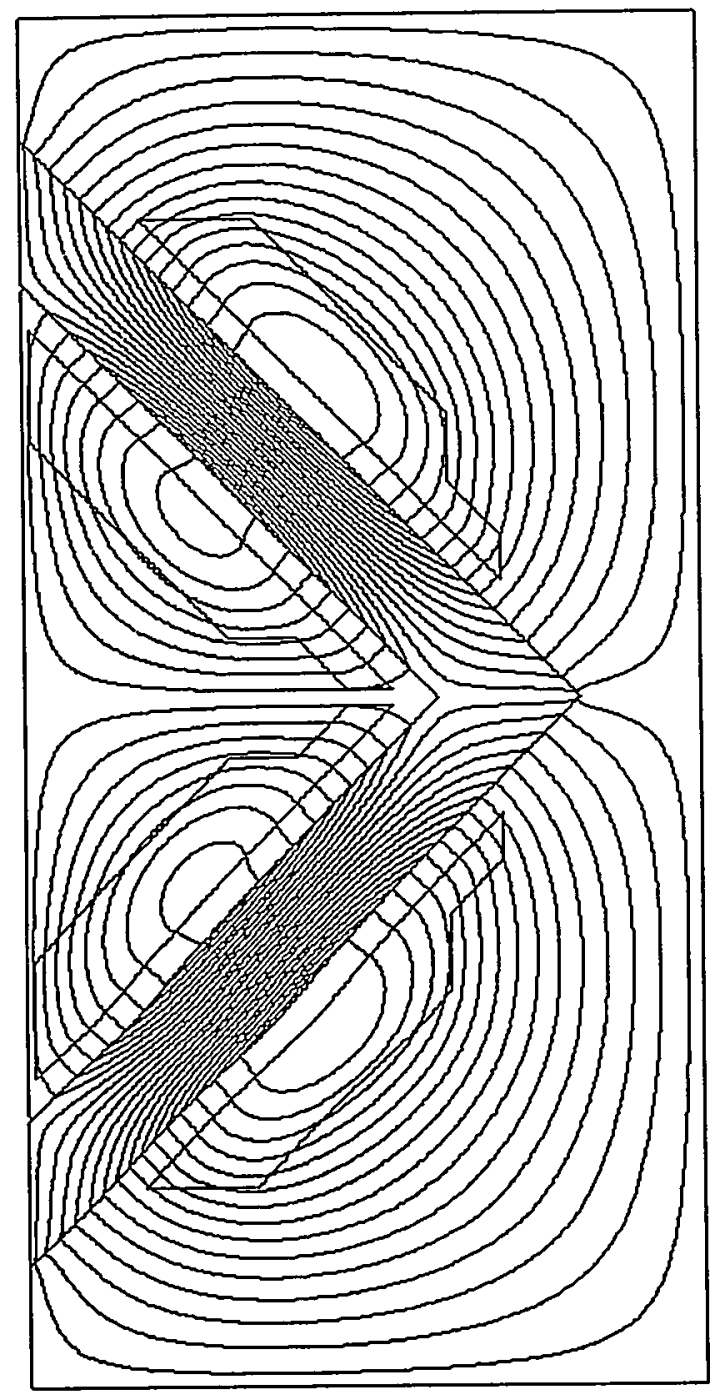


Figure 29: Electrical, mechanical and magnetic field characteristics of the prototype Panofksy skew magnet.

Magnet characteristics.

\begin{tabular}{|c|c|c|c|c|c|}
\hline & & \multicolumn{2}{|c|}{ predicted } & \multicolumn{2}{|c|}{ tested } \\
\hline & Units & skew & dipole & skew & dipole \\
\hline $\begin{array}{l}\text { Conductor } \\
\text { Current }\end{array}$ & A & $\begin{array}{l}3 / 16^{\prime \prime} \\
50\end{array}$ & $\begin{array}{r}712 \\
15\end{array}$ & $\begin{array}{c}3 / 16^{12} \\
50\end{array}$ & $\begin{array}{l}\# 12 \\
15\end{array}$ \\
\hline Mechanical & & & & & \\
\hline $\begin{array}{l}\text { Magnet length } \\
\text { Coil length / pole } \\
\text { Conductor area } \\
\text { Number of turns } \\
\text { Total current } \\
\text { Coil resistance } \\
\text { Voitage / coil } \\
\text { Total voltage } \\
\text { Current density }\end{array}$ & $\begin{array}{c}\mathrm{m} \\
\mathrm{m} \\
\mathrm{m} \mathrm{m}^{\wedge} 2 \\
\\
\mathrm{~A} \\
\text { Ohms } \\
\mathrm{V} \\
\mathrm{V} \\
\mathrm{A} / \mathrm{mm}^{\wedge} 2\end{array}$ & $\begin{array}{l}0.25 \\
46 \\
12.8 \\
81 \\
4050 \\
0.09 \\
4.5 \\
18.0 \\
3.9\end{array}$ & $\begin{array}{l}0.25 \\
70 \\
3.7 \\
126 \\
1890 \\
0.45 \\
6.8 \\
27.0 \\
4.1\end{array}$ & $\begin{array}{l}0.25 \\
46 \\
12.8 \\
81 \\
4050 \\
0.12 \\
6.0 \\
24.0 \\
-\end{array}$ & $\begin{array}{c}0.25 \\
70 \\
3.7 \\
126 \\
1890 \\
- \\
- \\
- \\
-\end{array}$ \\
\hline Cooling & & & & & \\
\hline $\begin{array}{l}\text { Cooling diameter } \\
\text { Temperature rise } \\
\text { Water flow } \\
\text { Pressure drop / coil }\end{array}$ & $\begin{array}{l}\text { in } \\
\operatorname{deg} \mathrm{C} \\
\mathrm{gal} / \mathrm{min} \\
\mathrm{PSI}\end{array}$ & $\begin{array}{l}0.125 \\
5 \\
0.18 \\
44\end{array}$ & $<1$ & $\begin{array}{c}7 \\
0.16\end{array}$ & $<1$ \\
\hline Field & & & & & \\
\hline $\begin{array}{l}\text { Field Non-Uniformity } \\
\text { Gradient Non-uniformity }\end{array}$ & $\%$ & $\begin{array}{c}- \\
19\end{array}$ & 2.5 & - & 2.0 \\
\hline
\end{tabular}


a stepping motor to a new location. This entire measuring procedure is automated using Labview software as the data acquisition software running on a MacIntosh.

Finally, Korolkov made a series of temperature measurements to estimate the cooling required as a function of magnet current for the prototype. Curves for temperature as a function of magnet current are shown in Fig. 31 for two different types of cooling circuit. The temperature is plotted relative to room temperature. The " $I^{2} R$ " nature of the dissipated power is obvious.

Korolkov will continue the design work on the Panofsky skew magnets and with building new prototypes.

\section{Drift Chamber Electronics Design}

The high peak luminosity expected in future CESR operations will demand that the inner layers of the CLEO III drift chamber be able to operate at a hit rate of around $10 \mathrm{kHz}$ per wire. This rate is too high for the present existing CLEO II electronics. New front-end electronics must be designed for the CLEO III drift chamber. This electronics has to work at the anticipated $10 \mathrm{kHz}$ hit rate per wire, has to provide a time resolution of $1 \mathrm{~ns}$ and provide a charge resolution of at least about $10 \%$.

SMU graduate student Igor Volobouev, together with a CLEO electronic engineer, has concentrated on designing the shaper circuit for the CLEO III front-end drift chamber electronics. He has designed a "time-over-threshold" (TOT) charge to time converter which produces a logic pulse whose width is proportional to the time the output signal from the drift chamber amplifier is above a pre-defined threshold. The circuit comprises a double integrator and a comparator. It is shown schematically in Fig.34. A double integrator was selected because the resulting TOT signal is insensitive to variations in the amplifier output and is relatively immune to pile up effects.

To estimate the charge resolution of the circuit, amplifier pulse shapes from a variety of sources were used as input to a simulation with SPICE programs of the TOT circuit. These sources included digitized pulses from a test drift chamber with no ambient magnetic field, an inner drift chamber inside CLEO II and the actual CLEO II drift chamber.

A typical pulse shape, integrator output and TOT circuit signal output are shown in Fig.33. The distribution of total charge $Q$ of the sample drift chamber pulses as a function of the output time over threshold for 1000 digitized sample pulses is shown on the left hand side of Fig.32. The $R C$ time constant of each of the integrators is $300 \mathrm{~ns}$. The solid line is a fit to the scatter plot. The relative deviation of the input charge and the inferred charge is plotted on the right hand side of Fig.32. The resulting charge resolution is $\sigma(\delta Q / Q)=7.5 \%$.

Since all test drift chamber pulses were obtained from a digitizing scope in self-triggering mode, there was no timing information to test the time resolution of the circuit. This will be done at a later date. The design described here is work in progress and represents only a partial solution for the CLEO III front-end drift chamber electronics. 
Figure 30: A schematic view of the test setup used to evaluate the magnetic field shape and strength of the prototype Panofsky skew magnet.

\section{Magnetic field measurement setup.}

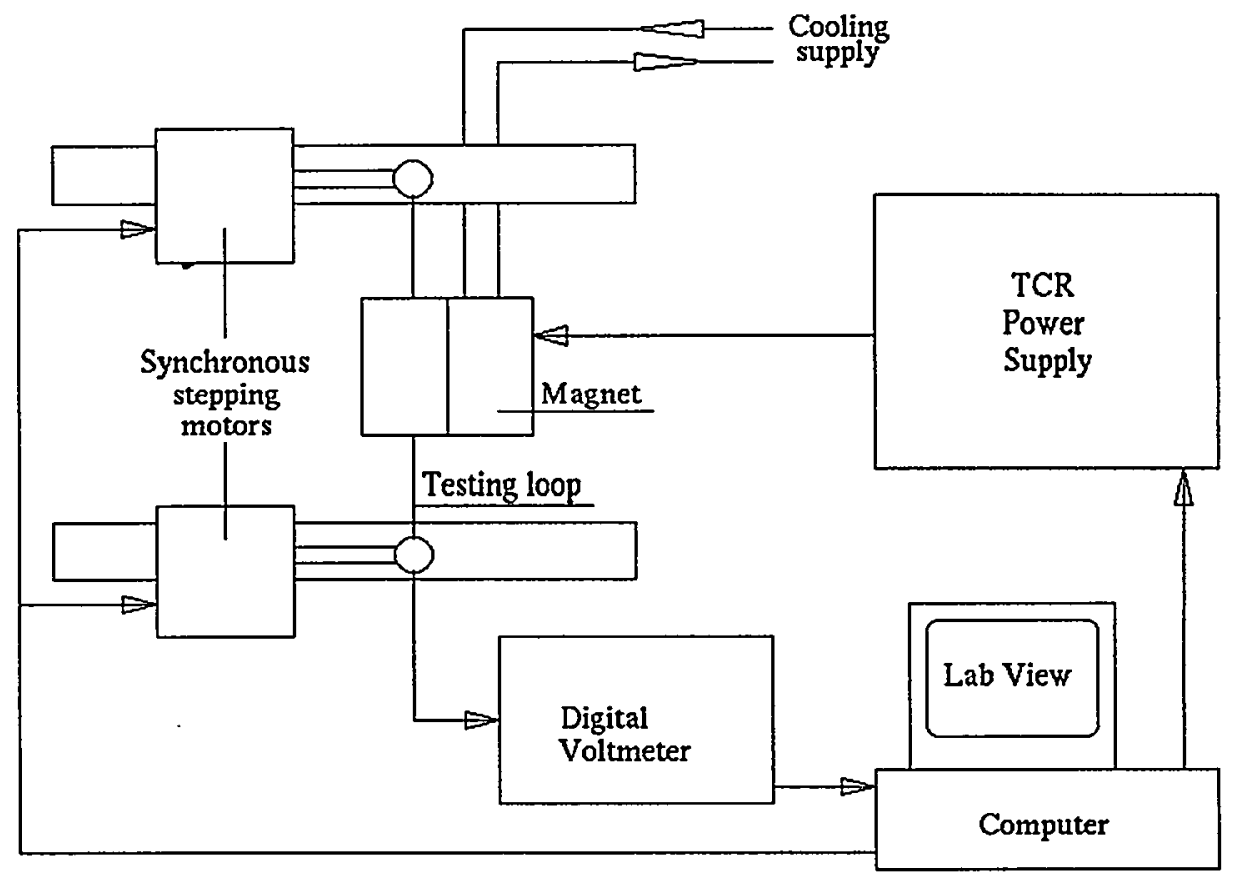


Figure 31: Temperature rise versus magnetic current for the prototype Panofsky skew magnet for two different cooling configurations.

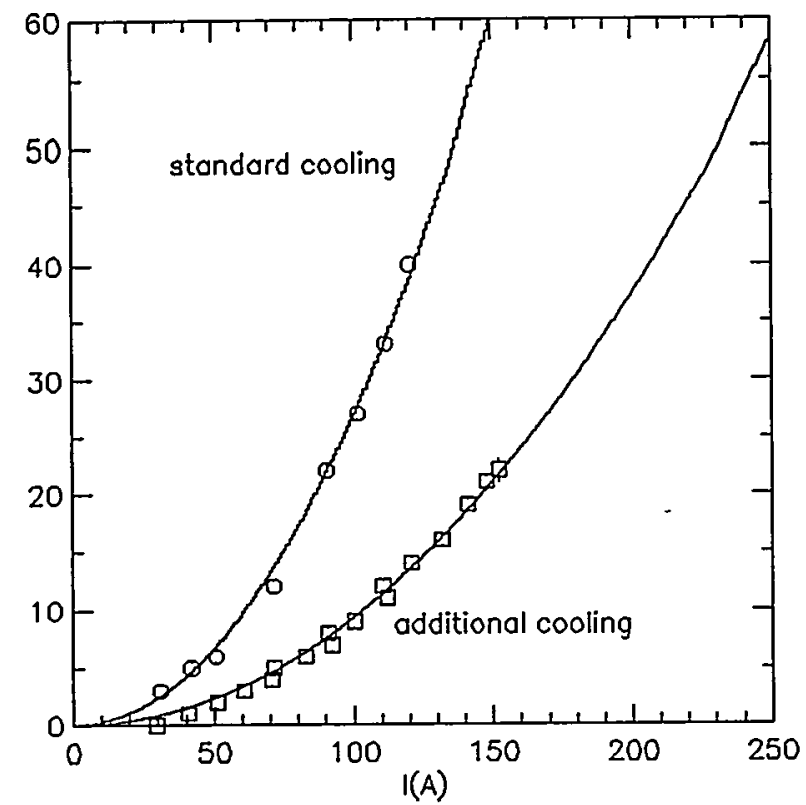


Figure 32: Schematic view of a proposed design for the shaper circuit of the CLEO III drift chamber front end electronics.
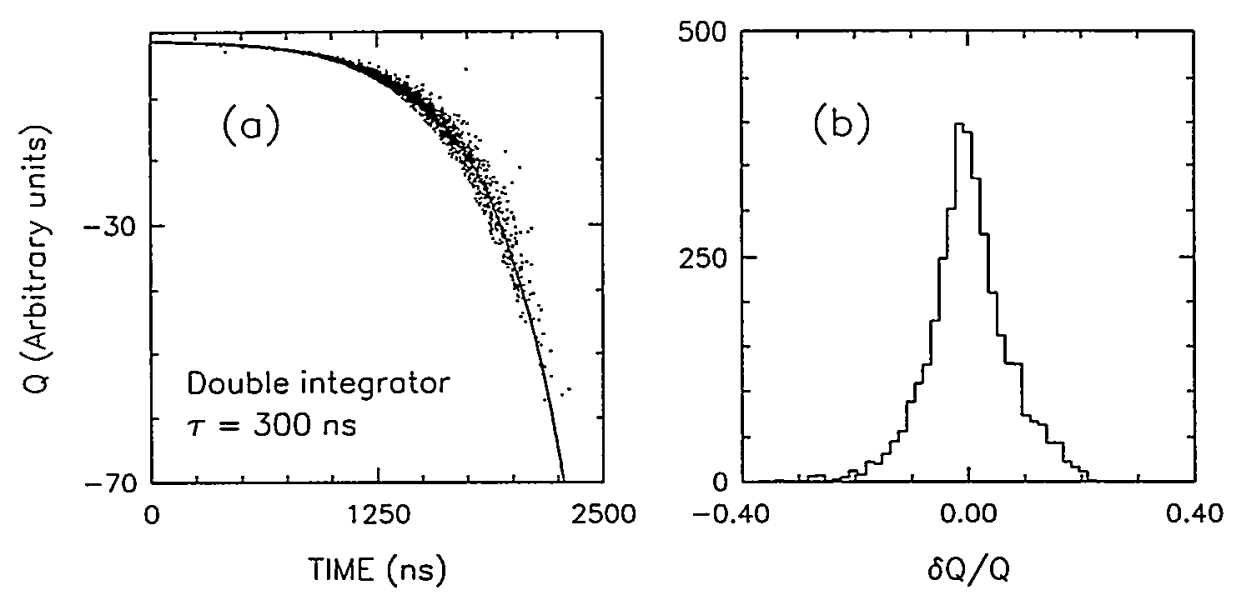

Time over threshold charge resolution:

(a) Actual total charge versus output pulse width together with fitted curve.

(b) Relative deviation of actual charge from the curve. Obtained resolution $\sigma(\delta Q / Q)=7.5 \%$. 
Figure 33: Input drift chamber pulse, shaped pulse and digital output pulse for a proposed CLEO III drift chamber shaper circuit.

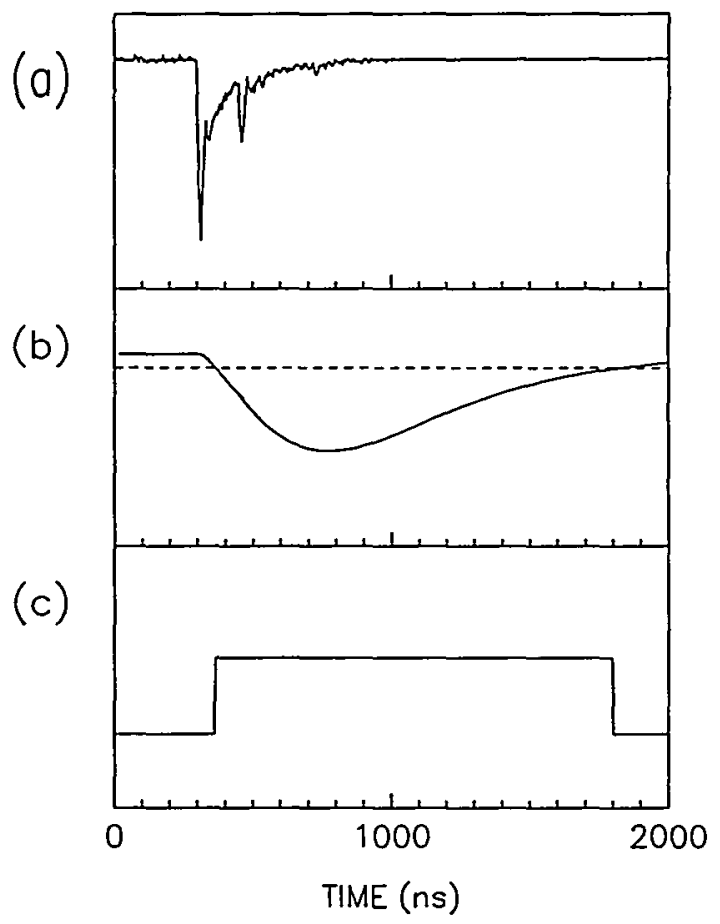

(o) A pulse from drift chamber wire.

(b) The same pulse shaped with double integrator. Time constant of each integrator is $300 \mathrm{~ns}$. Dotted line shows threshold value.

(c) Puise width over threshold. 
Figure 34: Charge resolution for a proposed shaper circuit for the CLEO III front end drift chamber electronics.

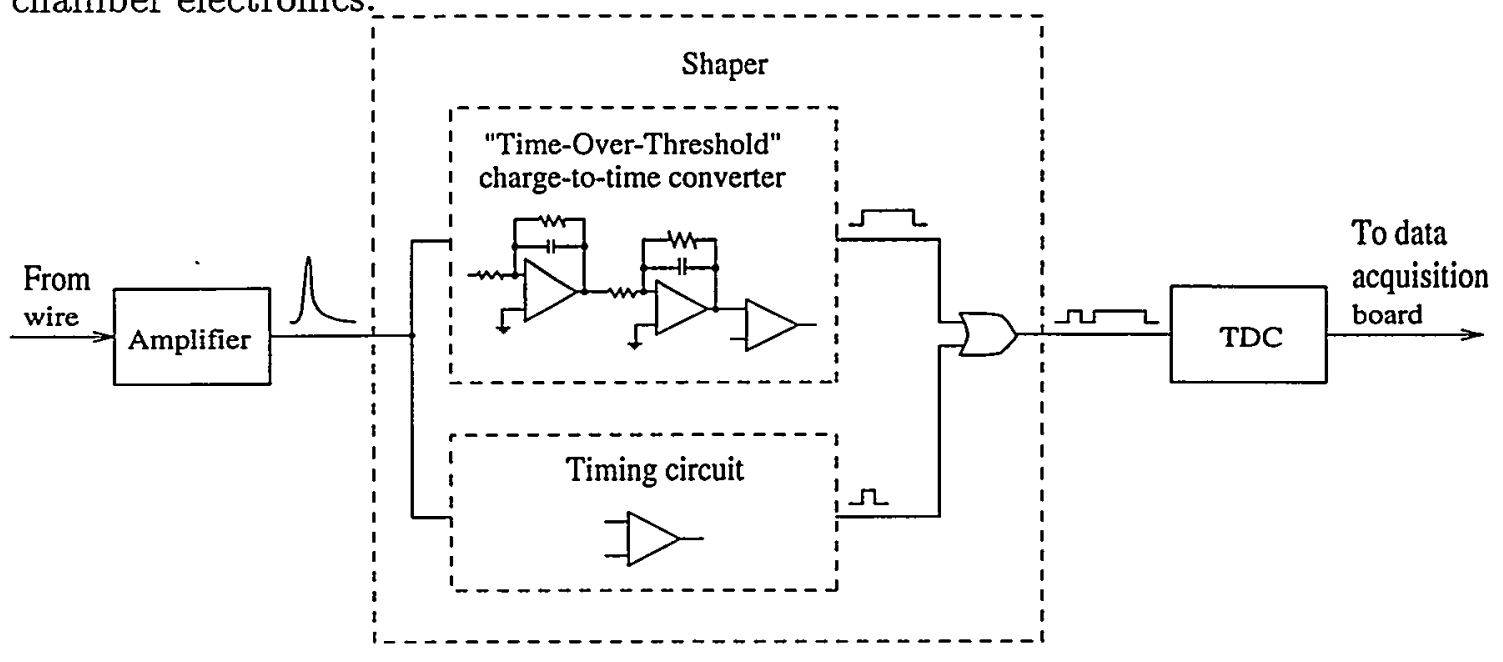

Possible design of drift chamber front-end electronic system based on time-over-threshold method. 


\section{ATLAS AT LHC}

The interest in physics at highest accessible accelerator energies was a prime motivation for the establishment of the SMU High Energy Physics group in Dallas close to the site of the SSC. After the closure of the SSC, the physics questions of the mechanism of the electroweak symmetry breaking remain unanswered. At present the LHC program represents a sole opportunity to explore the $\mathrm{TeV}$ mass scale in the next decade. The design parameters of the LHC are optimized for proton - proton collisions at a center of mass energy of $14 \mathrm{TeV}$ with the luminosity of $2.5 \cdot 10^{34} \mathrm{~cm}^{-2} \mathrm{~s}^{-1}$. The proton beam of about $2.8 \cdot 10^{14}$ particles is distributed around the LHC circumference in bunches of $10^{11}$ protons with a bunch arrival time interval of $25 \mathrm{~ns}$. There are several proton - proton interactions expected in each bunch crossing. Many physics goals of the SSC can be addressed at the LHC, though its lower $\mathrm{cm}$ energy and higher luminosity presents a greater experimental challenge for signal extraction.

The SMU group joined the ATLAS detector collaboration. ATLAS (A Toroidal LHC Apparatus) is a general purpose detector capable of precise measurement and identification of leptons, photons and jets. Its concept is based on having an outer superconducting toroid muon-magnet system and a central tracker surrounded by a solenoid coil. The artist view of the conceptual design of the experiment is shown in Fig.35. Starting from the beam line, the tracking system extends up to a radius of $1.2 \mathrm{~m}$ and is surrounded by a solenoid magnet. present design contains silicon tracking devices, gas microstrip detectors and small diameter drift tubes. Outside the solenoid, there is a hermetic calorimeter. Inner part of the calorimeter is measuring electromagnetic showers by sampling ionization deposited in liquid argon or liquid krypton. The design is following development of the GEM "accordion" calorimeter. The hadronic calorimeter has a scintillating tiles readout by wavelength shifting fibers. Forward calorimeter choice has not yet been finalized. Baseline option for the barrel section consists of a high pressure gas ionization calorimeter with plate structure in the front. A liquid argon device is proposed for endcaps. An integrated solution, copying the GEM approach of tungsten calorimeter with liquid argon readout, is under consideration. Outside the calorimeters there is muon spectrometer system for which the magnetic field is provided by a set of air-core toroidal magnets. A monitored drift tubes technology has been chosen for the muon chambers. It will be complemented by honeycomb cathode strip chambers and jet chambers. A special challenge facing the muon chambers is the design of the alignment system that can provide a chamber position accuracy on the order of $20 \mu$. The design of the detector is still evolving. The Technical Design Report will be completed in December 1994. Within the ATLAS collaboration the SMU group plans to work together with other US groups on the liquid argon (krypton) calorimetry. Here, the experience gained in the process of optimization of GEM detector is directly applicable. In particular, the US ATLAS groups will work on the design of the electrodes, feedthroughs, readout electronics and barrel cryostat. Initially SMU will work together with the BNL and the University of Rochester groups on the design and optimization of the barrel cryostat and on the system integration. This will include study of the effects of the magnetic field on the cryostat and the simulation of the detector responses. In addition we plan to assist other ATLAS groups in the design and construction of a full size prototype of the electromagnetic calorimeter and 
to participate in the beam test. A section of the US ATLAS proposal describing the liquid calorimetry work is provided in Appendix 1.

Figure 35: View of the proposed ATLAS detector.

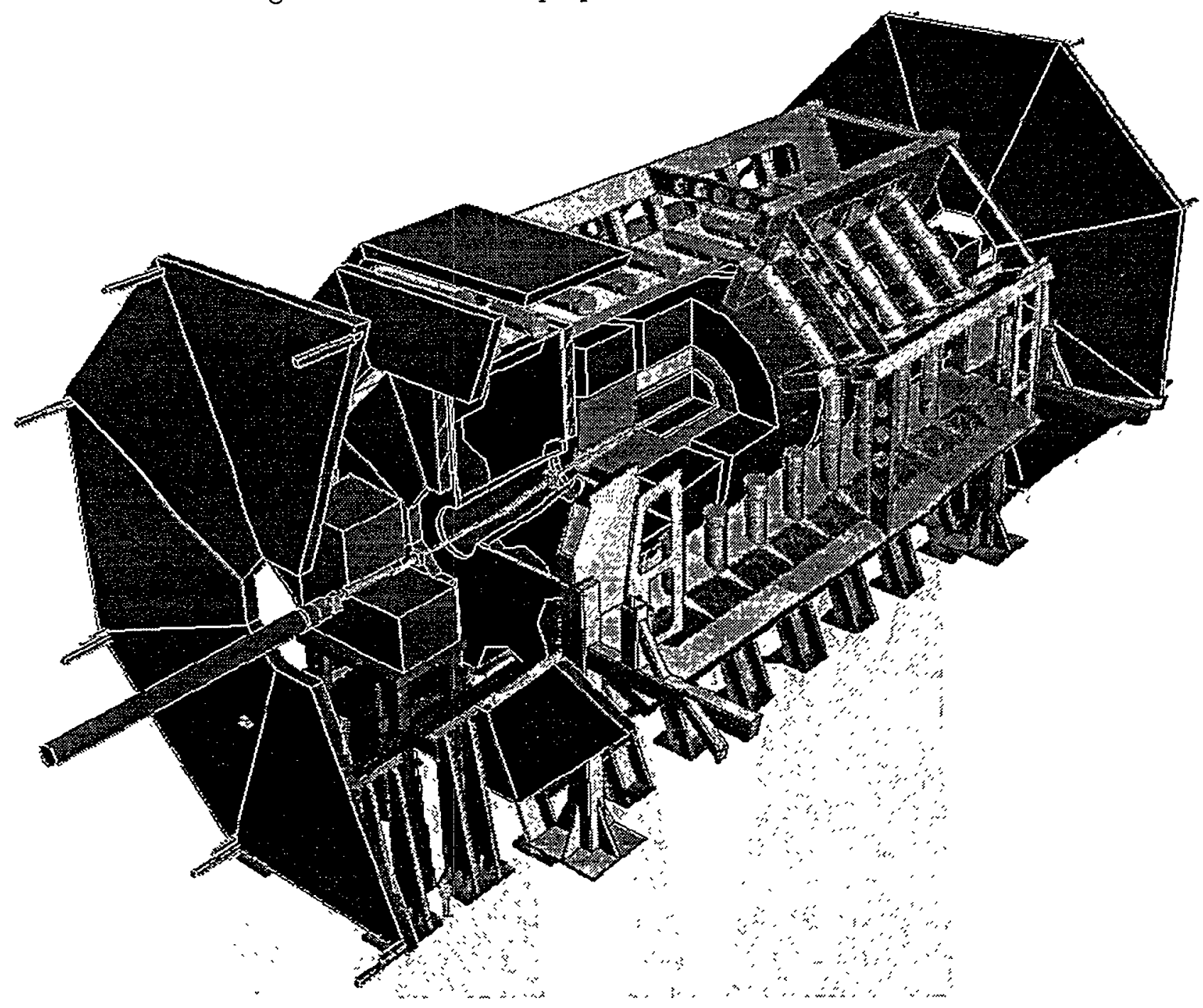




\section{LIGHT VELOCITY IN MAGNETIC FIELD}

The closure of the SSC project left many of the accelerator components available for other uses. The Expression of Interest generated by the SMU group in collaboration with reserchers from Jet Propulsion Laboratory in Pasadena, California and from SSC Laboratory to study light retardation in a strong magnetic field has been selected for "Project Definition Study" by the DOE. The idea is to use the SSC Accelerator Systems String Test facility of up to 10 dipole magnets together with "state-of-the-art" laser interferometry techniques to measure the effective index of refraction and rotation of polarisation during light passage through the magnetic field. There are two physics motivations for such measurements. The first is to test the higher order QED effects due to vacuum polarization. Quantum Electrodynamics treats the vacuum as a sea of virtual particle pairs. A polarization of such pairs under a strong magnetic field can cause the refractive index of vacuum to deviate from unity. For a dipole magnetic field, the effect can be observed as a birefringence of light i.e., a difference of light velocities depending on the direction of polarization with respect to the field direction. The effect is very small and has not yetbeen observed experimentally.

The second and potentially much more interesting motivation is generated by a possibility of observing a deviation from the QED birefringence expectations. Such deviation is expected in the case of axion, or any pseudoscalar particle that couples to two photons. Photon axion mixing occurs only for the photon polarization parallel to external magnetic field and thus enhances the birefringence effect. The original concept has been suggested in 1979 by Iacoppini and Zavattini and has been followed by the BNL - Fermilab - Rochester Trieste Collaboration which used two CBA magnets to set weak limits on axion- photon coupling. Present study suggest that the higher field strength and much longer length of the SSC magnets together with much improved optical technique may provide several orders of magnitude higher sensitivity to an axion coupling than that available in past measurements. In addition, a novel idea of using a resonant photon - axion transition by adding low presure helium gas in the path of the photon is being explored.

At present the newly formed collaboration of Caltech - JPL - SMU - Taiwan physicists considers an option of experiment where $10 \mathrm{SSC}$ dipoles with the total field length of 150 $\mathrm{m}$ are bracketed by high finesse Fabry-Perot interferometer. Interferometry studies done by groups working on the gravitational wave detection e.g., LIGO, indicate that finesse of up to $10^{5}$ is within reach of present day technology. In a possible arrangement, shown in Fig.36, two orthogonal polarization direction of light share the common path. The light source will be provided by a Nd:YAG laser. Systematic effects will be controlled by a modulation of the magnetic field between the nominal value of $6.76 \mathrm{~T}$ and a lower value of about $3 \mathrm{~T}$ and by the modulation of the polarization of light. Other optics schemes, including heterodyne measurements (see Fig.37) are also under consideration.

We expect that the "Project Definition Study" will conclude at the end of October, 1994 with an experiment proposal. 
Figure 36:

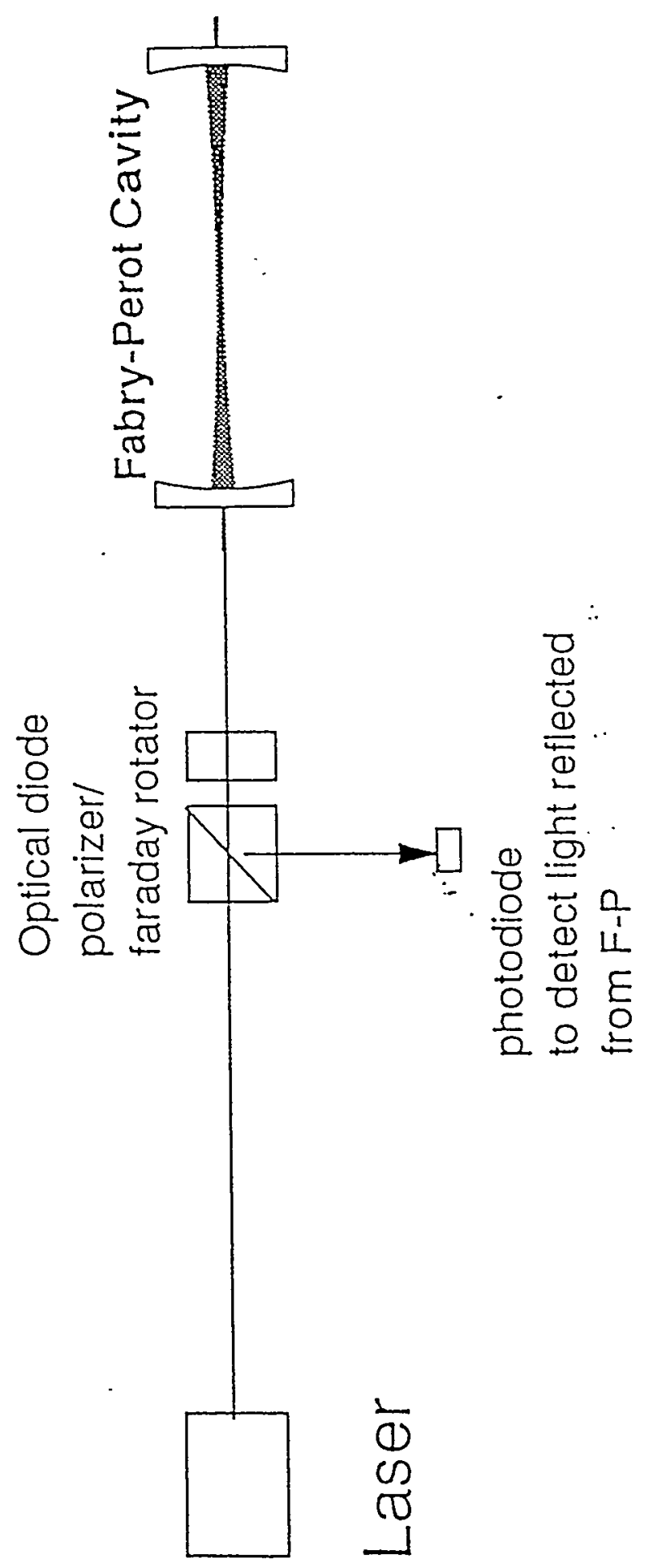


Figure 37:

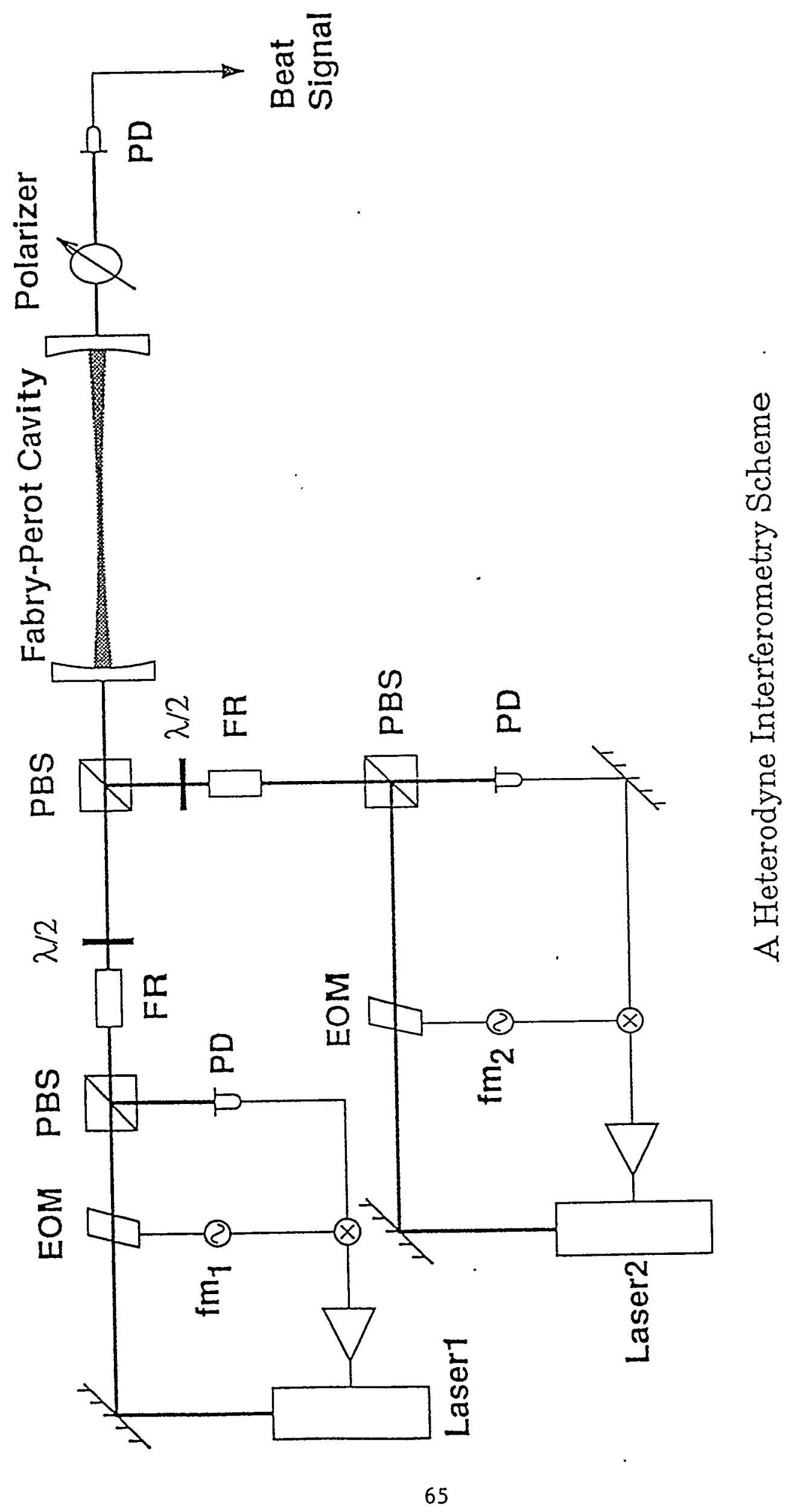




\section{List of publications $1993-1994$}

\subsection{Publications in Refereed Journals}

1. The tau lepton and its neutrino.

R. Stroynowski and A. J. Weinstein, Annual Review of Nuclear and Particle Physics 1993 43: 457-528.

2. Upsilon spectroscopy: transitions in the bottomonium system.

D. Besson and T. Skwarnicki, Annual Review of Nuclear and Particle Physics 1993 43: 333-378.

3. A Test of Optimized Perturbative QCD to $\mathcal{O}\left(\alpha_{s}^{2}\right)$ and $\mathcal{O}\left(\alpha_{s}^{3}\right)$ from $Z^{0}$ and $\tau$ Decays, and a Method to Deal with the Renormalization Scale Ambiguity S. Sanghera, International Journal of Modern Physics, A9 (1994).

4. Tau decays with one charged particle plus multiple $\pi^{0}$ s. M. Procario et al., The CLEO Collaboration. Physical Review Letters 70 (1993) 1207.

5. Lepton asymmetry measurements in $\bar{B} \rightarrow D^{*} l^{-} \bar{\nu}_{l}$ and implications for V-A and the form factors.

S. Sanghera et al., The CLEO Collaboration. Physical Review D47 (1993) 791.

6. Search for exclusive $b \rightarrow u$ semileptonic decays of $\mathbf{B}$ mesons.

A. Bean et al., The CLEO Collaboration.

Physical Review Letters 70 (1993) 2681.

7. A measurement of the tau lepton mass.

R. Balest et al., The CELO Collaboration.

Physical Review D47 (1993) 3671.

8. A limit on the tau neutrino mass.

D. Cinabro et al., The CLEO Collaboration.

Physical Review Letters 70 (1993) 3700.

9. Evidence for penguin-diagram: First observation of $B \rightarrow K^{*}(892) \gamma$.

R. Ammar et al., The CLEO Collaboration.

Physical Review Letters 71 (1993) 674.

10. Production and decay of the $D_{s 1}(2536)^{+}$.

J. P. Alexander et al., The CLEO Collaboration.

Physics Letters B 303 (1993) 377.

11. Measurement of the ratio $B\left(D^{+} \rightarrow \pi^{0} l^{+} \nu\right) / B\left(D^{+} \rightarrow \overline{K^{0}} l^{+} \nu\right)$.

M. S. Alam et al., The CLEO Collaboration.

Physical Review Letters 71 (1993) 1311.

12. Two measurements of $B^{0} \bar{B}^{0}$ mixing.

J. Bartelt et al., The CLEO Collaboration.

Physical Review Letters 71 (1993) 1680. 
13. Measurement of the decay $\tau^{-} \rightarrow \pi^{-} \pi^{+} \pi^{-} 2 \pi^{0} \nu_{\tau}$.

D. Bortoletto et al., The CLEO Collaboration.

Physical Review Letters 71 (1993) 1791.

14. Measurement of the $D \rightarrow \pi \pi$ branching fractions.

M. Selen et al., The CLEO Collaboration.

Physical Review Letters 71 (1993) 1973.

15. Study of the decays $\Lambda_{c}^{+} \rightarrow \Xi^{0} K^{+}, \Lambda_{c}^{+} \rightarrow \Sigma^{+} K^{+} K^{-}$and $\Lambda_{c}^{+} \rightarrow \Xi^{-} K^{+} \pi^{+}$.

P. Avery et al., The CLEO Collaboration.

Physical Review Letters 71 (1993) 2391.

16. Measurement of the absolute branching fraction for $D^{0} \rightarrow K^{-} \pi^{+}$.

D. S. Akerib et al., The CLEO Collaboration.

Physical Review Letters 71 (1993) 3070.

17. Observation of the charmed baryon $\Sigma_{c}^{+}$and measurement of the isospin mass splittings of the $\Sigma_{c}$.

G. Crawford et al., The CLEO Collaboration.

Physical Review Letters 71 (1993) 3259.

18. Measurement of exclusive $\Lambda_{c}$ decays with a $\Sigma^{+}$in the final state.

Y. Kubota et al., The CLEO Collaboration.

Physical Review Letters 71 (1993) 3255.

19. Study of $D^{0}$ decays into $\bar{K}^{0}$ and $\overline{K^{* 0}}$.

M. Procario et al., The CLEO Collaboration.

Physical Review D 48 (1993) 4007.

20. Observation of $B^{0}$ decay to two charmless mesons.

M. Battle et al., The CLEO Collaboration.

Physical Review Letters 71 (1993) 3922:

21. Measurement of charmless semileptonic decays of B mesons.

J. Bartelt et al., The CLEO Collaboration.

Physical Review Letters 71 (1993) 4111.

22. Measurement of exclusive semileptonic decays od $\mathbf{D}$ mesons.

A. Bean et al., The CLEO Collaboration.

Physics Letters B 317 (1993) 647.

23. Search for exclusive $b \rightarrow u$ transitions in hadronic decays of $\mathrm{B}$ mesons involving $D_{s}^{+}$and $D_{s}^{*+}$ mesons.

J. P. Alexander et al., The CLEO Collaboration.

Physics Letters B 319 (1993) 365.

24. Observation of $D^{0} \rightarrow K^{+} \pi^{-}$.

D. Cinabro et al., The CLEO Collaboration.

Physical Review Letters 72 (1994) 1406.

25. Observation of a new charmed strange meson.

Y. Kubota et al., The CLEO Collaboration.

Physical Review Letters 72 (1994) 1972.

26. Measurement of the branching fraction for $D^{+} \rightarrow K^{-} \pi^{+} \pi^{+}$.

R. Balest et al., The CLEO Collaboration.

Physical Review Letters 72 (1994) 2328. 
27. Analysis of hadronic transitions in $\Upsilon(3 S)$ decays.

F. Butler et al., The CLEO Collaboration.

Physical Review D 49 (1994) 40.

28. Study of the decay $\Lambda_{c}^{+} \rightarrow \Lambda l^{+} \nu_{l}$.

T. Bergfeld et al., The CLEO Collaboration.

Physics Letters B 323 (1994) 219.

29. First measurement of $\Gamma\left(D_{s}^{+} \rightarrow \mu^{+} \nu\right) / \Gamma\left(D_{s}^{+} \rightarrow \phi \pi^{+}\right)$.

D. Acosta et al., The CLEO Collaboration.

Physical Review D 49 (1994) 5690.

30. Search for $B^{0}$ decays to two charged leptons.

R. Ammar et al., The CLEO Collaboration.

Physical Review D 49 (1994) 5701.

31. Measurement of the branching fraction $B\left(\tau^{ \pm} \rightarrow h^{-} \pi^{0} \nu_{\tau}\right)$.

M. Artuso et al., The CLEO Collaboration.

Physical Review Letters 72 (1994) 3762.

32. Exclusive hadronic $\mathbf{B}$ decays to charm and charmonium final states.

M. S. Alam et al., The CLEO Collaboration.

Physical Review D 50 (1994) 43.

33. Luminosity measurement with the CLEO II detector.

G. Crawford et al., The CLEO Collaboration.

Nuclear Instruments and Methods in Physics Research A 345 (1994) 429.

34. A measurement of $B\left(D_{s}^{+} \rightarrow \phi l^{+} \nu\right) / B\left(D_{s}^{+} \rightarrow \phi \pi^{+}\right)$.

F. Butler et al., The CLEO Collaboration.

Physics Letters B 324 (1994) 255.

35. Study of the five-charged-pion decays of the $\tau$ lepton.

D. Gibaut et al., The CLEO Collaboration.

Physical Review Letters 73 (1994) 934.

36. Measurement of Cabibbo-suppressed decays of the $\tau$ lepton.

M. Battle et al., The CLEO Collaboration.

Physical Review Letters 73 (1994) 1079.

37. Production and decay of $D_{1}(2420)^{\circ}$ and $D_{2}^{*}(2460)^{0}$.

P. Avery et al., The CLEO Collaboration.

Physics Letters B 331 (1994) 236.

38. Observation of $\Lambda_{c}^{+}$decays to $\Lambda \pi^{+} \pi^{0}, \Sigma^{0} \pi^{+}, \Sigma^{0} \pi^{+} \pi^{0}$, and $\Sigma^{0} \pi^{-} \pi^{+} \pi^{+}$.

P. Avery et al., The CLEO Collaboration.

Physics Letters B 325 (1994) 257.

\subsection{Conference Talks}

1. Forbidden decays of $\tau$ lepton from CLEO.

R. Stroynowski, Representing the CLEO Collaboration.

Proceedings of the International EPS Concerence on High Energy Physics, Marseille, France, July 22-28, 1993, page 143.

Editions Frontieres, J. Carr and M. Perrottet editiors. 
2. Exclusive and rare $\tau$ lepton decays.

R. Stroynowski

Proceedings of the International EPS Concerence on High Energy Physics, Marseille, France, July 22-28, 1993, page 484.

Editions Frontieres, J. Carr and M. Perrottet editiors.

3. Axion Detection,

R. Stroynowski

Talk presented at Snowmass'94 Workshop, "Particle and Nuclear Astrophysics and Cosmology in the Next Millennium, Snowmass, CO, June 29-July 14, 1994.

4. Measurement of Spectral Moments in Tau Decays

R. Stroynowski

Talk presented at the Parallel Session Pa-11 at XXVII International Conference on High Energy Physics, Glasgow, Scotland, July 20-27, 1994.

5. Summary of Tau'94

R. Stroynowski

Summary of the Third Workshop on Tau Lepton Physics, Montreux, Switzerland, 19-22 September 1994.

6. Status of $\tau$ Physics

T. Skwarnicki

Review talk presented at the Joint Meeting of the American Physical Society and the American Association of Physics Teachers, Crystal City, VA, 18-22 April, 1994.

7. Summary of Tau Physics Results.

T. Skwarnicki,

Summary of parallel session presented at the $8^{\text {th }}$ Meeting of the Division of Particles and Fields of the American Physical Society, Albuquerque, New Mexico, 2-6 August, 1994.

8. First Measurement of Inclusive $b \rightarrow s \gamma$ Rate.

T. Skwarnicki, Representing the CLEO collaboration.

Talk presented at the Joint Fall Meeting of the Texas Section of the APS, AAPT, and Zone 13 of the SPS, University of Texas at Austin, Austin, TX, 14-15 October, 1994.

9. A Vision of QCD at CLEO.

S. Sanghera

DPF QCD Working Group Meeting, University of Wisconsin-Madison, April 9-10, 1994.

10. Experimental Tests of Strong Physics at CLEO II, S. Sanghera International Symposium on Radiative Corrections, Gatlinburg, TN, June27-July 1, 1994.

11. Measurement of Hadronic Spectral Moments in Tau Decays and a Determination of $\alpha_{s}$,

S. Sanghera, Representing the CLEO collaboration

Talk presented at $8^{\text {th }}$ Meeting of the Division of Particles and Fields of the American Physical Society, Albuquerque, New Mexico, 2-6 August, 1994.

12. $b \rightarrow c$ Results from Threshold Experiments.

M. Lambrecht 
Talk Presented at the "Physics in Collision" conference, St.Petersburg, Florida, May 1994.

13. Search for Neutrinoless Decays of $\tau$ Lepton,

I. Volobouev

Talk presented at the Joint Meeting of the American Physical Society and the American Association of Physics Teachers, Crystal City, VA, 18-22 April, 1994.

\subsection{Conference Papers}

1. Overview of the superconducting magnet subsystem for the GEM detector at the SSC.

G. Deis et al.,

Submitted to the 5th IISSC Symposium, San Francisco, Ca., May 1993.

"Supercollider 5", SSCL-PREPRINT 233 (1993).

2. Conductor design for the GEM detector magnet.

J. V. Minervini et al.,

Submitted to the 5th IISSC Symposium, San Francisco, Ca., May 1993. "Supercollider 5", SSCL-PREPRINT 348 (1993).

3. Papers submitted to the European Physical Society conference on High Energy Physics, Marseille, July 22-29, 1993.

The CLEO Collaboration.

1. Search for neutrinoless decays of the tau into three leptons.

2. Search for $\tau \rightarrow \phi \pi \nu_{\tau}$.

3. Study of the five charged pion decay of the $\tau$ lepton.

4. Measurement of the cross section fro $\gamma \gamma \rightarrow \bar{p} p$.

5. Measurement of the two-photon width of the $\chi_{c 2}$ at CLEO II.

6. Determination of $\alpha_{s}$ from a measurement of the direct photon spectrum in $\Upsilon(1 S)$ decays.

7. Observation of the excited charmed baryon $\Lambda_{c}^{*}$ at CLEO II.

8. Evidence for new decay modes of the $\Xi_{c}^{+}$.

9. Search for the $\Omega_{c}^{0}$ in $e^{+} e^{-}$annihilations.

10. A study of the decays $\Lambda_{c} \rightarrow \Lambda l \nu_{l}$.

11. Observation of $D^{0} \rightarrow K^{+} \pi^{-}$.

12. Measurement of the absolute branching fraction for $D^{0} \rightarrow K^{-} \pi^{+}$.

13. Investigation of the $c \bar{s}$ meson vector-pseudoscalar mass splitting.

14. $D^{* * 0}$ production and decay.

15. First measurement of $\Gamma\left(D_{s} \rightarrow \mu^{+} \nu\right) / \Gamma\left(D_{s} \rightarrow \phi \pi^{+}\right)$.

16. Measurement of exclusive semileptonic decays of $\mathrm{D}$ mesons.

17. The ratio $B \rightarrow D^{* 0} l \nu_{l} / B \rightarrow D^{*+} l \nu_{l}$.

18. Observation of $B^{0}$ decay to two charmless mesons.

19. Inclusive measurement of $B$ meson semileptonic branching fractions.

20. A measurement of the charged to neutral $B$ meson lifetime ratio.

21. Measurement of charmless semileptonic decays of $B$ mesons. 
4. Papers submitted to the Lepton-Photon conference, Cornell, August 10-13, 1993.

1. Search for neutrinoless decays of the tau into three leptons.

2. Search for $\tau \rightarrow \phi \pi \nu_{\tau}$.

3. Study of the five charged pion decay of the $\tau$ lepton.

4. Measurement of the cross section fro $\gamma \gamma \rightarrow \bar{p} p$.

5. Measurement of the two-photon width of the $\chi_{c 2}$ at CLEO II.

6. Determination of $\alpha_{s}$ from a measurement of the direct photon spectrum in $\Upsilon(1 S)$ decays.

7. A measurement of the $\eta_{c}$ two-photon decay width.

8. $\Upsilon(1 S) \rightarrow \gamma$ plus non-interacting particles.

9. Evidence for new decay modes of the $\Xi_{c}^{+}$.

10. Search for the $\Omega_{c}^{0}$ in $e^{+} e^{-}$annihilations.

11. A study of the decays $\Lambda_{c} \rightarrow \Lambda l \nu_{l}$.

12. Observation of $D^{0} \rightarrow K^{+} \pi^{-}$.

13. Measurement of the absolute branching fraction for $D^{0} \rightarrow K^{-} \pi^{+}$.

14. Investigation of the $c \bar{s}$ meson vector-pseudoscalar mass splitting.

15. $D^{* * 0}$ production and decay.

16. First measurement of $\Gamma\left(D_{s} \rightarrow \mu^{+} \nu\right) / \Gamma\left(D_{s} \rightarrow \phi \pi^{+}\right)$.

17. Measurement of exclusive semileptonic decays of $D$ mesons.

18. Observation of a new excited charmed baryon $\Lambda_{c}^{*}$ at CLEO II.

19. Observation of a new excited charmed baryon.

20. Measurement of the decay asymmetry parameters in decays $\Lambda_{c}^{+} \rightarrow \Lambda \pi^{+}$and $\Lambda_{c}^{+} \rightarrow \Sigma^{+} \pi^{0}$.

21. Observation of of $\Lambda_{c}$ decays to $\Lambda \pi^{+} \pi^{0}, \Sigma^{0} \pi^{+}, \Sigma^{0} \pi^{+} \pi^{0}$, and $\Sigma^{0} \pi^{-} \pi^{+} \pi^{+}$.

22. Measurement of exclusive $\Lambda_{c}^{+}$decay modes containing protons and anti-kaons.

23. An absolute measurement of $B\left(D^{+} \rightarrow K^{-} \pi^{+} \pi^{+}\right)$.

24. The ratio $B \rightarrow D^{* 0} l \nu_{l} / B \rightarrow D^{*+} l \nu_{l}$.

25. Observation of $B^{0}$ decay to two charmless mesons.

26. Inclusive measurement of $B$ meson semileptonic branching fractions.

27. A measurement of the charged to neutral $B$ meson lifetime ratio.

28. Measurement of charmless semileptonic decays of $B$ mesons.

29. Search for the decay $\overline{B^{0}} \rightarrow \pi^{+} l^{-} \overline{\nu_{l}}$.

30. Search for the exclusive charmless decays $B \rightarrow \phi X_{s}$, with $X_{s}=K^{+}, K_{s}^{0}, K^{*+}, K^{* 0}$ or $\phi$.

31. A measurement of $B\left(\bar{B}^{0} \rightarrow D^{*+} l \bar{\nu}_{l}\right)$.

32. Exclusive $B$ decays to charm and charmonium final states.

33. Inclusive decays of $B$ mesons to $J / \Psi$ and $\Psi$ '.

5. The superconducting solenoid magnet system for the GEM detector at the SSC.

G. Deis et al.

SSCL-504 (1993). Submitted to 13th International Conference on Magnet Technology, Victoria, Canada, September 20-24, 1993.

6. Papers submitted to the XXVII International Conference on High Energy 
Physics, Glasgow, Scotland, July 22-28, 1994.

The CLEO Collaboration.

CONF94-1 First measurement of the inclusive rate for the radiative penguin decay $b \rightarrow s \gamma$.

CONF94-2 A constraint on $\left|V_{t d} / V_{t s}\right|$ from $B \rightarrow \rho(\omega) \gamma / B \rightarrow K^{*} \gamma$.

CONF94-3 Search for exclusive charmless hadronic $B$ decay modes.

CONF94-4 search for $B \rightarrow K^{+} l^{-}$and $B \rightarrow K^{*} l^{+} l^{-}$decays.

CONF94-5 A search for $B \rightarrow \tau \nu$.

CONF94-6 Measurement of the branching ratio of $B \rightarrow X e \nu$ with lepton tags.

CONF94-7 Measurement of the form factors for $B^{0} \rightarrow D^{*+} l^{-} \nu$.

CONF94-8 A study of baryon production in $B$ decay: search for semileptonic decays of $\mathrm{B}$ mesons to charmed baryons and the first observation of $\Xi_{c}$ production in $\mathrm{B}$ decay.

CONF94-9 Measurements of $B \rightarrow D_{s}^{+} X$ decays.

CONF94-11 Inclusive decays of $B$ mesons to charmonium.

CONF94-12 The inclusive decay $B \rightarrow \eta X$.

CONF94-13 Evidence for exclusive B decays to final states containing a charmed baryon.

CONF94-14 Search for CP violation in $D^{0}$ decay.

CONF94-15 Search for $D^{0} \rightarrow K^{+} \pi^{-} \pi^{0}$.

CONF94-16 Observation of $D_{1}(2430)^{+}$and $D_{2}^{*}(2470)^{+}$.

CONF94-17 New decay modes of the $\Lambda_{c}^{+}$charm baryon.

CONF94-18 Measurement of the ratios $B\left(D_{s}^{+} \rightarrow \eta l^{+} \nu\right) / B\left(D_{s}^{+} \rightarrow \phi l \nu\right)$ and $B\left(D_{s}^{+} \rightarrow\right.$ $\left.\eta^{\prime} l^{+} \nu\right) / B\left(D_{s}^{+} \rightarrow \phi l^{+} \nu\right)$.

CONF94-19 Form factor ratio measurement in $\Lambda_{c}^{+} \rightarrow \Lambda e^{+} \nu_{e}$.

CONF94-20 Measurement of the tau lepton lifetime.

CONF94-21 $\pi^{-} \pi^{+}$energy correlation in tau pair events.

CONF94-22 Measurement of $B\left(\tau^{-} \rightarrow h^{-} h^{+} h^{-}\left(\pi^{0}\right) \nu_{\tau}\right)$.

CONF94-23 Study of the decays $\tau^{-} \rightarrow K^{0} h^{-}\left(\pi^{0}\right) \nu_{\tau}$.

CONF94-24 Measurement of $B\left(\tau^{-} \rightarrow \nu_{\tau} K^{-} K^{0}\right)$ and $B\left(\tau^{-} \rightarrow \nu_{\tau} K^{-} K^{0} \pi^{0}\right)$.

CONF94-25 Observation of tau decays with two neutral kaons.

CONF94-26 Measurement of hadronic spectral moments in tau decays and a determination of $\alpha_{s}$.

CONF94-27 Measurement of the tauonic branching ratio of the $\Upsilon(1 S)$.

CONF94-28 A Study of jet production rates in the four flavor continuum and a test of QCD.

\subsection{Seminars and Colloquia}

1. GEM Detector, R. Stroynowski, Colloquium at Cornell University, Ithaca, NY, 1993.

2. $\Upsilon$ Spectroscopy,

T. Skwarnicki 
Colloquium at Cornell University, Ithaca, NY, 1994.

3. First measurement of inclusive $b \rightarrow s \gamma$ rate by CLEO-II,

T. Skwarnicki

Seminar at DESY, Hamburg, Germany, 1994.

4. First measurement of inclusive $b \rightarrow s \gamma$ rate by CLEO-II.,

T. Skwarnicki

seminar at Fermilab, Batavia, IL, 1994.

5. First measurement of inclusive $b \rightarrow s \gamma$ rate by CLEO-II.,

T. Skwarnicki

seminar at Brookhaven National Lab., Long Island, NY, 1994.

6. Aerogel R\&D Results from SMU,

J. Dominick

seminar at Caltech, Pasadena, CA, 1994.

7. Status of Perturbative QCD and determination of Strong Coupling Constant.

S. Sanghera

Colloquium at Cornell University, Ithaca, NY, 1993.

\subsection{Proposals and Technical Notes}

1. Report to the PAC.

The CLEO Collaboration. January 25, 1993.

2. GEM Technical Design Report.

W. C. Lefmann et al., The GEM Collaboration.

SSCL-SR-1219, GEM-TN-93-262 (1993).

3. Tracking the $\mathbf{B}$ mass shift.

M. Lambrecht

CLEO Note CBX 93-33 (1993).

4. Accessing the fully reconstructed B tags at CLEO.

M. Lambrecht, N. Katayama, G. Kalbfleisch and S. Ball.

CLEO Note CBX 93-34 (1993).

5. A measurement of the charged to neutral $B$ meson lifetime ratio.

M/ Lambrecht, B. Gittelman, G. Kalbfleisch and N. Katayama.

CLEO Note CBX 93-35 (1993).

6. Search for neutrinoless decays of tau into three leptons.

I. Volobouyev, T. Skwarnicki and R. Stroynowski.

CLEO Note CBX 93-48 (1993).

7. Proposal for a Data Run for $\Upsilon$ Spectroscopy,

D. Besson, J. Domominick, T. Skwarnicki.

CLEO Note CBX 93-55 (1993).

8. First Observation of the $\Upsilon(1 D)$ States,

T. Skwarnicki

CLEO Note CBX 93-98 (1993). 
9. Upgrades and staging possibilities for GEM.

N. Baggett, B. Barish, J. Brau, H. Gordon, D. Marlow, Yu. Kamyshkov, L. Rosenson, G. Sanders, R. Stroynowski and W. Willis.

GEM Note to Program Advisory Committe of the Superconducting Supercollider Laboratory, May 1993.

10. Detector for a B-factory.

R. Kass et al., The CLEO Collaboration.

Conceptual design of the B-factory detector. Submitted to the Program Advisory Committee of the Cornell Laboratory of Nuclear Science, June 1993.

11. Feasibility study of a silica aerogel Cerenkov counter for CLEO III.

S. Alam (SUNY at Albany), G. Masek, H. Paar (UC San Diego), P. Skubic (University of Oklahoma), T. Skwarnicki, R. Stroynowski (SMU)

Submitted to CLEO III Steering Committee. December 1993.

12. Search for neutrinoless decays of the tau lepton.

I. Volobouev, T. Skwarnicki and R. Stroynowski.

CLEO Note CBX 94-1 (1994).

13. Semileptonic branching fractions and lifetime ratio of $B^{-}$and $\bar{B}^{0}$.

M. Lambrecht, M. Saulnier, G. Vasseur and K. Kinoshita.

CLEO Note CBX 94-18 (1994).

14. Measurement of Inclusive $b \rightarrow s \gamma$

T. Skwarnicki

CLEO Note CBX 94-19 (1994).

15. Initial GEANT simulation studies of aerogel PID device for CLEO III. V. Fadeyev, T. Skwarnicki and R. Stroynowski. CLEO note CBX 94-31 (1994).

16. The CLEO III Detector: Design and Physics Goals.

The CLEO Collaboration.

CLNS94/1277 (1994).

17. The CLEO III Detector Upgrade Project.

D. H. Miller et al., The CLEO Collaboration.

Proposal to the Department of Energy, February 1994.

18. Light retardation in a high magnetic field and search for light scalar/pseudoscalar particles using ultra-sensitive interferometry.

Talso C. P. Chui et al., Caltech - SMU - Stanford - SSCL - Tsing Hua University, Taiwan - Chiao Tung University, Taiwan.

Expression of Interest for the Use of the SSC Assets. Submitted to the U. S. Department of Energy, April 1994.

SMUHEP 94-08 (1994).

19. Comment on tau physics in the next decade.

Tomasz Skwarnicki and Ryszard Stroynowski.

Position paper contributed to the DPF study on the future of High Energy Physics. SMU HEP 94-06. April 1994.

20. Definition studies for a proposal to measure the velocity of light in a magnetic field. 
JPL - SMU - SSCL - Taiwan Collaboration.

Proposal submitted to the U. S. Department of Energy, JPL 80-4004, May 1994.

\subsection{Theses}

1. Study of resonance structures in $\tau \rightarrow 4 \pi \nu_{\tau}$.

V. Shelkov, Master degree thesis. SMU HEP 93-11.

2. Measurement of $\operatorname{BR}\left(\tau \rightarrow h^{-} \nu_{\tau}\right)$.

P. Zadorozhny, Master degree thesis. SMU HEP 93-12.

3. Search for neutrinoless decays of tau into three leptons.

I. Volobouev, Master degree thesis. SMU HEP 93-10.

4. Study of gas mixtures for large muon chambers.

J. Pryor, Senior thesis. SMU HEP 94-05.

\subsection{Papers submitted for publication or in preparation}

1. Measurement of Cross section for $\gamma \gamma \rightarrow p \bar{p}$.

M. Artuso et al., The CLEO Collaboration.

CLNS 93/1245, CLEO 93-17 (1993). Submitted for publication.

2. Measurement of two-photon production of the $\chi_{c 2}$.

V. Shelkov et al., The CLEO Collaboration.

CLNS 93/1250, CLEO 93-19 (1993). Submitted for publication.

3. Observation of inclusive $B$ decays to the charmed baryons $\Sigma_{c}^{++}$and $\Sigma_{c}^{0}$.

M.Procario et al., The CLEO Collaboration.

CLNS 93/1264, CLEO 93-24 (1993). Submitted for publication.

4. Precision measurement of the $D_{s}^{+*}-D_{s}^{+}$mass difference.

D. N. Brown et al., The CLEO Collaboration.

CLNS 94/1271, CLEO 94-6 (1994). Submitted for publication.

5. Two-photon production of charged pion and kaon pairs.

J. Dominick et al., The CLEO Collaboration.

CLNS 94/1274, CLEO 94-9 (1994). Submitted for publication.

6. Measurement of the $\vec{B} \rightarrow D^{*} l \bar{\nu}$ branching fractions and $\left|V_{c b}\right|$.

B. Barish et al., The CLEO Collaboration.

CLNS 94/1285, CLEO 94-13 (1994). Submitted for publication.

7. Search for neutrinoless decays of the tau lepton.

J. Bartelt et al., The CLEO Collaboration.

CLNS 94/1287, CLEO 94-15 (1994). Submitted for publication.

8. Semileptonic branching fractions of charged and neutral $\mathbf{B}$ mesons.

M. Athanas et al., The CLEO Collaboration.

CLNS 94/1286, CLEO 94-16 (994). Submitted for publication.

9. A Comparative Study of Cluster Jet Algorithms in the Energy Range from CESR to LEP

S. Sanghera

Submitted to Phys. Rev. D. 
10. Axion search with optical technique.

R. Stroynowski and Ariel Zhitnitsky.

SMU-HEP 94-12 (1994). 


\section{Current and Pending Support}

Current support of the experimental group at SMU consists of the Department of Energy Division of High Energy Physics grant DE-FG05-92ER40722 of 200K\$; an Outstanding Junior Investigator award to prof. Skwarnicki of $75 \mathrm{~K} \$$ and a supplement of $25 \mathrm{~K} \$$ for the initial support of prof. Coan. Additional funding is provided by the DOE SSC Division grant for the Project Definition Study of the light velocity measurement in magnetic field. This grant is administered by Jet propulsion Laboratory in Pasadena, California with a subcontract to SMU for $26 \mathrm{~K} \$$.

Formely, our group recieved additional funding from the Texas National Research Laboratory Comission $(90 \mathrm{~K} \$$ for the experimental group out of $120 \mathrm{~K} \$$ for SMU Physics Department) and from the SSC Laboratory (100K\$ for prof. Skwarnicki). We hope to obtain some capital equipment funds for our future technical undertakings on the CLEO III and ATLAS experiments.

\section{$9 \quad$ Facilities and Resources}

With the help of the TNRLC funds we have set up a laboratory suitable for the design, prototyping and testing of the detector components. This laboratory contains a CAMAC based fast data acquisition system controlled by the Labview software running on the SUN SPARCstation and a cosmic ray telescope. In addition, substantial amount of electronic, laser and optical equipment is available. So far, this laboratory has been used primarily for the aerogel Cerenkov detector R\&D study. We also have a small but adequate machine shop and this year we have been able to recruit an exceptional machinist/designer from the SSC laboratory. The University has provided additional laboratory space for the research activities. In a near future we plan to use this space for the detector construction activities associated with CLEO III upgrade and for setting up of electronics shop facility. The computing facilities are described in the following section.

\section{Experimental Computing}

The computing system for the Experimental High Energy Physics group at SMU was set up with the help of capital equipment DOE grants adding up to $\$ 48,000$ and the additional $\$ 14,000$ included in Skwarnicki's Outstanding Junior Investigater award. SMU provided an initial ethernet connection and access to a CISCO router free of charge. The present configuration, shown in Fig.38, consists of 2 DECstations and 1 DEC Alpha machine together with distributed disks, tape drives, printers and terminals. Three additional small DEC workstations support SMU postdoctoral fellows and students resident at the CLEO experiment at Cornell. These computing resources are insufficent for the complexity of data analyses required by CLEO II data. CLEO data set comprises now over $20004 \mathrm{~mm}$ tapes. Even selected "small" data sets e.g., the tau data skim, consist of over 200 tapes. In addition, similar size general purpose Monte Carlo data are also necessary. This saturates all 
available resources while the CLEO collaboration anticipates an increase of the number of collected events by a factor of 5 over the next 3 years. There are three areas of computing, in particular, that need improvement at SMU:

- CPU power: The SMU group has increased in size significantly since its inception and the richness of available data allows for increased number and variety of physics analyses. Specialised Monte Carlo data sets require substantially larger CPU power than that available at present.

- Data handling resources: Improvement of storage technology stimulated a change of standard tape storage within the CLEO collaboration towards the newly available 10 Gigabytes DLT tapes. Additional disk storage for data files is also needed.

- Network improvements: The increased size of the group and available data requires modification of networking arrangement. The SMU campus wide ethernet network is saturated by the large I/O rate of CLEO data analysis. The High Energy Group computing system needs to be separated from the rest of the campus and form e.g, local FDDI token ring.

Additional planned modiffications include terminal server, fast printer and remote dial-in modem capabilities.

List of Hardware and Pricing for Proposed Equipment.

\begin{tabular}{|lcrrrc||}
\hline \multicolumn{1}{|c}{ Hardware } & Vendor & List Price & $\begin{array}{c}\text { Educational } \\
\text { Discount Price }\end{array}$ & Quantity & Sub Total \\
\hline \hline Alpha AXP 3000/600 & DEC & $23,000.00$ & $18,000.00$ & 2 & $36,000.00$ \\
\hline \hline 9GB Disk Drive & ADS & 3800.00 & with discount & 3 & $11,400.00$ \\
\hline \hline Terminal Server & DEC & $5,000.00$ & 3000.00 & 1 & $3,000.00$ \\
\hline \hline Tape Drives & DEC & $4,000.00$ & $3,000.00$ & 2 & $6,000.00$ \\
\hline \hline FDDI Controller & DEC & $2,400.00$ & 2000.00 & 5 & $10,000.00$ \\
\hline \hline FDDI Concentrator & DEC & $30,000.00$ & $25,0000.00$ & 1 & $25,000.00$ \\
\hline \hline 19" X-terminal & NCD & $5,500.00$ & $3,500.00$ & 2 & $7,000.00$ \\
\hline \hline Laser Printer & HP & $6,500.00$ & $4,500.00$ & 1 & $4,500.00$ \\
\hline \hline Misc & Multi-Vendor & $6,000.00$ & with discount & - & $6,000.00$ \\
\hline \hline
\end{tabular}


Figure 38:

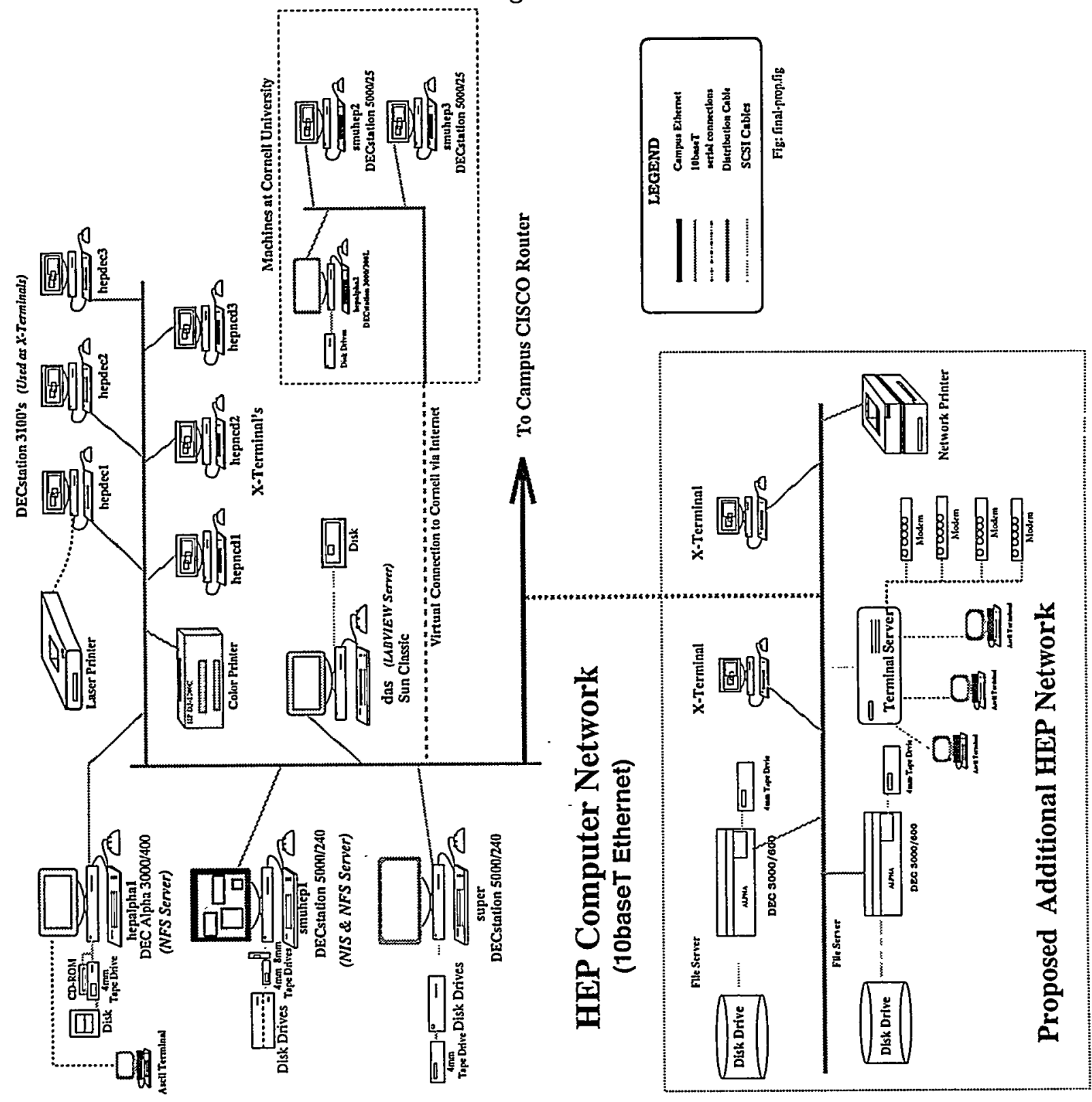




\section{Budget Explanation}

The budget included in this proposal includes support for the continuation of present activities at CLEO II and for the additional activities on the construction of CLEO III and ATLAS experiments. An expansion of the scope of activities has been made possible by an addition of a third SMU faculty member to our group.

\section{Personnel}

In addition to current personnel (Faculty: Coan, Skwarnicki, Stroynowski; Postdoctoral Research Associates: Dominick, Lambrecht, Sanghera and Graduate Students: Fadeyev, Korolkov, Li, Shelkov, Volobouev, Wei and Zadorozhny) we anticipate a need for additional postdoctoral research associate who will concentrate on ATLAS related work. Five of the graduate students concentrate full time on research. During the R\&D and detector construction stages we expect a need for a help provided by undergraduate students. At present the prevailing wages for the SMU undergraduates are at the level of $6 \$$ per hour. The request for technical support covers activities of machinist/designer at $50 \%$ level with another $50 \%$ coming from the University funds and for $75 \%$ of the computer manager with the remaining $25 \%$ included in the theory group request. The secretarial and administrative support will be provided by the University.

\section{Travel}

The travel request is split into two tasks: CLEO II together with CLEO III activities and ATLAS. The CLEO experiment collects data for about 11 month per year. Frequent trips to Cornell for the collaboration meetings, shifts and physics analysis group meetings are necessary. We estimate a need for 4 trips/yr average. Occasional attendance at conference to present CLEO results is also budgeted.

The travel related to ATLAS experiment will include foreign travel mainly to CERN for ATLAS collaboration meetings called 5 times per year and for detector development related

meetings in other European countries. The liquid calorimetry work of the US ATLAS group is concentrated at BNL and we project the travel for prototype related work there at 8 trips per year total.

\section{Equipment}

The request for computing equipment is justified in the section on experimental computing. A table with a list of equipment and pricing is included. The additional equipment 
request is for the power supply for a 2 Tesla magnet owned by SMU. This magnet has been on loan at SSC laboratory and after return needs a new power supply. We plan to use this magnet to test effects of the magnetic field on various components of the CLEO III and ATLAS detectors. 


\section{Appendix 1: Liquid Calorimetry Part of the US ATLAS Proposal}

\subsection{Introduction}

The ATLAS noble liquid calorimeter is designed to incorporate many unique features. The electromagnetic (EM) section uses an absorber/electrode design based on the accordion concept. The ATLAS EM calorimeter will have the following properties: i) very good energy resolution $(\triangle E / E=10 \% / \sqrt{E} \oplus 0.7 \%)$ to facilitate the detection of the decays $H^{0} \rightarrow \gamma \gamma$ and $H^{0} \rightarrow Z Z^{*} \rightarrow e^{+} e^{-} e^{+} e^{-}$for a narrow Higgs; ii) transverse and longitudinal segmentation to allow the rejection of multiphoton backgrounds; iii) photon pointing to the interaction vertex $(\Delta \theta / \theta=60 \mathrm{mrad} \sqrt{E}+0.6 \mathrm{mrad})$, both to improve $\gamma \gamma$ mass resolution and to aid in hadron/electron discrimination; iv) excellent position resolution $(4.4 \mathrm{~mm} / \sqrt{E})$; and v) good timing resolution ( $5 \mathrm{GeV} \times n s e c / E$ for a $5 \times 5$ tower analog sum). The performance of the EM calorimeter is particularly critical to ATLAS achieving its physics goals, and the US groups working in this area are investigating the optimization of the detector together with questions relating to overall experiment integration, access, installation, radiation levels, and shielding. These studies are being carried out in close coordination with other ATLAS groups.

The ATLAS endcap hadronic calorimeter will employ liquid argon technology because of its radiation hardness. The endcap calorimeters cover about two units of rapidity (approximately from $|\eta|=1.6$ to $|\eta|=3.6$ ). The hadronic modules are behind about 1.8 interaction lengths of electromagnetic calorimetry. The hadronic calorimeter is constructed in two sections along the beam axis, with each section containing about 4.5 interaction lengths. The modules in each of these sections form projective towers with a transverse segmentation of $\Delta \eta \times \Delta \phi=0.1 \times 0.1$. The absorber plates are $2.5 \mathrm{~cm}$ thick copper and the argon gaps are $0.8 \mathrm{~cm}$. The gap signals are read out using a 2-stage electrostatic transformer (EST) made with Kapton electrodes separated by Hexcell. The design is very similar to that developed for GEM except that there are no copper tiles in the ATLAS EST. We are working closely with other ATLAS groups to optimize the design of the hadronic endcaps.

The ATLAS calorimeter will incorporate new features essential for operation at very high luminosities, which distinguish its design from the ones employed in previous high energy collider calorimeters (SLD, $D \emptyset$ and $H 1$ ). The high luminosity environment places great demands on the calorimeter's readout and trigger electronics. A principal emphasis of the US effort in the liquid calorimeter subsystem will be to assume major responsibility for defining and optimizing the calorimeter readout. We have unique expertise in this area within our group, and can make substantial contributions to defining and optimizing this system. In particular, we have recently developed cryogenic preamplifiers, a novel calibration system, and a switched capacitor array (SCA) readout with better than 12 bit precision. We note that V. Radeka (BNL) has been appointed to be responsible for the integration of the ATLAS calorimeter electronics, which includes specifying and certifying the calorimeter readout chain.

Calorimeter readout systems also require significant cryogenic development - in par- 
ticular, cryostat design and fabrication, including the design of high density feedthroughs. There is also the need for extensive radiation damage and noble liquid contamination studies. We have a great deal of relevant experience in these areas, and plan to assume major responsibilities for these activities.

\subsection{Experience of US Groups}

The US groups that propose to participate in the design and construction of the ATLAS noble liquid calorimeters have been in the forefront of $R \& D$ on this technology from its inception, including its initial use in R806, E272, NA34, E706, D $\emptyset$ and SLD, and the proposed extension of this technology to higher energy and higher luminosity colliders. In the last five years, under the auspices of the SSC generic and detector specific R\&D programs, these groups have developed a broad range of knowledge and expertise culminating in the selection of noble liquid calorimetry by GEM at the SSC. This work has kept the United States in the forefront of this technology, particularly with respect to achieving high speed of response and precise energy resolution. It will enable the US groups involved to address the technical issues for which they propose to take responsibility in a coherent and coordinated manner, and thereby significantly contribute to developing and building the ATLAS noble liquid calorimeters.

\subsection{Major Tasks of US Groups}

We describe below the principal elements of the technical tasks for which the US groups propose to take major responsibility.

Calorimeter Readout Electronics The calorimeter readout chain includes all electronics and interconnections from the Kapton electrodes through the Level I \& II triggers and the data acquisition system (DAQ). It is the heart of the EM calorimeter and to a major extent determines its performance. The electronic readout of liquid calorimetry poses special challenges to the design and construction of such systems. Principal items that are unique to this technology are: a) front end electronics that need to operate at cryogenic temperatures and in a high radiation environment; b) a calibration system adequate to exploit the unique strengths of this technology; and c) a precision readout able to handle a dynamic range of 16 bits at a sampling rate of $40 \mathrm{MHz}$. We list below the main components of the readout chain (see also the later section on Feedthroughs):

Electrode Design The readout electrodes are complicated from both a design and a manufacturing point of view. We have considerable experience in this area at BNL, gained in part through our participation in GEM and in the CERN RD3 collaboration. The experience we have gained and relevant manufacturing capability in the United States will be an important asset to the construction of the ATLAS calorimeter, even though primary responsibility for providing this aspect of the system is expected to lie elsewhere. 
Summing and Motherboards The signals from the electrodes need to be summed before they arrive at the preamps and calibration motherboards. The motherboard design is constrained by the fact that the calibration system and the preamps occupy the same real estate. In addition, the design of the ATLAS EM calorimeter requires a very high density readout - e.g., an integrated preshower detector would have as many as 32 channels in a $20 \times 20 \mathrm{~cm}^{2}$ region. Minimizing cross-talk and noise pickup requires a precisely engineered connection scheme due to the large number of channels involved.

Calibration The precision and uniformity of its calibration system to a large extent determine the performance one can hope to achieve from a calorimeter. We have proposed that ATLAS employ an innovative calibration system developed at BNL, which is based on a distribution of $\mathrm{DC}$ currents. This system will also be used to calibrate the trigger sums and to exercise the trigger electronics.

Preamps Speed and noise requirements force one to place the preamps in the cold liquid. This imposes special requirements on these units since they not only need to operate at cryogenic temperatures, but also to function reliably in a high radiation region that is essentially inaccessible. We have many years of experience in the design and fabrication of such cold preamps. In the forward region, where shaping times reach $20 \mathrm{nsec}$, the preamps can be located on the outside of the cryostat.

Cable Driver Because of dynamic range requirements (16 bits) and the need to transmit signals as much as 50 meters to the outside of the detector, one requires a design where the noise pickup by the cables is small compared to the system least count. To address this, we are developing a cable driver that will linearly amplify signals using different scale factors above and below $\sim 150 \mathrm{GeV}$.

Digitizing Board The calorimeter signals are passed through a shaper/SCA/ADC sequence and undergo data compression before being sent to the Level I \& II triggers and the DAQ. These functions are all performed on the digitizing board, the main components of which are:

Shapers The calorimeter signal processing chain will need to operate over a dynamic range of 16 bits, requiring high and low gain paths operating in parallel. The shaping time will vary from $20-70 \mathrm{nsec}$, depending on the location of the output channel.

SCA's We plan to use 2 SCA's, each having a 12 bit accuracy, to cover the full dynamic range of the calorimeter. The SCA's will need to store information at a rate of $40 \mathrm{MHz}$, and transfer data to the $A D C$ at a rate of $500 \mathrm{kHz}$.

ADC's Once a Level I trigger has been satisfied, all the analog signals from the SCA's are digitized for input to the Level II trigger and the DAQ. Up to 5 samples in time will be digitized per channel and the information passed to the digitized signal processor (DSP) for further analysis. We will employ a 12 bit ADC, whose speed will determine the number of channels that can be multiplexed. 
Electronics Certification Center We plan to establish an electronics certification center at BNL and Nevis capable of testing all of the different components of the electronics chain of the ATLAS liquid calorimeter. This work will lead to detailed specifications and subsequent certification procedures for the electronic components to be used in the calorimeter readout. We expect this center to ultimately become part of the experiment's overall electronics certification process.

Feedthroughs The calorimeter signals leave the cryostat through a set of feedthroughs that carry the signals from the cold region across the insulating vacuum to a warm environment. These feedthroughs need to maintain signal fidelity, while minimizing crosstalk and noise pickup and satisfying the cryogenic requirements of minimal heat loss and the maintenance of a reliable vacuum. The development of suitable feedthroughs for signal lines, calibration, power, and monitoring purposes is therefore an important part of cryogenic technology where both we and US industry have exceptional capabilities. We thus propose to take responsibility for the design and construction of the high density ATLAS feedthroughs.

The University of Washington group has designed and produced (originally for GEM) prototype feedthroughs with 480 pins each that have been tested at Orsay, and found to meet the ATLAS requirements for hermeticity and pin density at the highest applicable cryostat pressures. A similar version with 960 pins that also meets the space requirements for ATLAS has been designed and ordered. Mating cable connectors have been designed and fabricated in order to provide a complete system.

The University of Rochester group is investigating an alternative feedthrough scheme in which flat signal cables are directly soldered into a feedthrough flange: For this approach to be successful, one has to be able to manufacture cables that do not leak along their signal lines, as well as a vacuum-tight connection between the cable ground and the flange. Initial tests have been encouraging, and the group has designed and produced a prototype test flange for testing under high vacuum, both in a warm and in a cryogenic environment.

Calorimeter Signal Processing One problem which needs careful consideration is the processing of data from the cryogenic calorimeter. For a variety of reasons, it is advantageous in collider applications to record data in the form of several (up to 5) time samples for every calorimeter channel. These data can then be processed in the online electronics to extract the amplitude and time origin of the signal and to apply calibration constants. Although an enormous number of computations is required, these calculations can be carried out in the front end electronics with many digital signal processors working in parallel. Construction of such a system for a precision calorimeter requires that the processing algorithms be well understood at the time of the system design. Examples of the sort of questions that need study are: the number and position of the samples; how information from two ADC scales will be combined; how the presence of out-of-time energy deposits will be detected; how the various sources of noise will be tracked; and how the calibration system will be used to provide information on the shape of the wave form and its time offset, as well as its gain calibration. Data taken using the 1992 BNL test calorimeter have provided the first opportunity to study some of these problems. The 1994 CERN test and subsequent RD3 
test runs are expected to yield valuable additional test data for the continuation of these studies.

Calorimeter Trigger System The ATLAS calorimeter trigger will be based on "trigger towers", consisting of a $4 \times 4$ matrix of individual channels. There will be a total of approximately 7500 trigger channels. The trigger sums will be digitized in 8-bit flash ADC's, and the data will be digitally processed to produce a trigger decision. Elements contributing to the trigger include isolated electrons/photons, jets, and missing $E_{T}$. Some of the Monte Carlo work that has been done for GEM in optimizing the trigger algorithms will be of direct use in ATLAS, and we plan to continue working on these problems.

At the technical level, our participation in the development of the calorimeter trigger will primarily be to focus on problems connected to the formation of the analog sums, which involves the proper grouping of channels on motherboards and cables, developing techniques for the selection of preamplifiers of similar gain, understanding timing problems, and designing summing amplifiers. We will also need to understand the ways in which the calibration system can be used to equalize the gains of trigger sums over the entire calorimeter.

An important function of the first level trigger will be to determine the bunch crossing of interest. The technique to be used for this purpose has not yet been selected. One of the techniques being considered is to utilize a special purpose constant fraction discriminator which was tested in the 1992 BNL run. We employed an improved version of this discriminator in the 1994 CERN beam test, with the goal of evaluating its performance for use in ATLAS as the bunch crossing identifier.

Barrel Cryostat The performance of the EM calorimeter is strongly influenced by the design of the barrel and endcap cryostats. Consequently, EM calorimeter optimization necessarily involves optimizing the design of these cryostats. This includes examining such important issues as choosing the most appropriate length and radius of the barrel cryostat, as well as selecting the optimum construction material - i.e., aluminum or stainless steel. The barrel cryostat also incorporates the signal feedthroughs, and is thus an integral component of the EM calorimeter readout chain. Finally, the barrel cryostat is a significant cost item, and it is thus appropriate for the US groups who wish to play a major role in designing and constructing the EM calorimeter to take responsibility for providing it. This responsibility must be closely coordinated with the other ATLAS groups who will build the endcap cryostats and the solenoid that will share the barrel cryostat and its associated cryogenics.

The Rochester group, in collaboration with BNL, has begun to evaluate the preliminary ATLAS barrel cryostat design. To pursue this activity, Rochester plans to strengthen its existing engineering manpower, which presently includes one senior and one junior mechanical engineer, by adding an experienced cryogenics engineer. In addition to seeking ways to minimize interference between the magnet coil supply piping and the EM calorimeter, we will focus particular attention on issues involving the calorimeter readout.

Over 100k signal lines, as well as power lines and high voltage cabling, will have to be connected to feedthroughs that span the insulating vacuum of the cryostats. The design and 
placement of the barrel feedthroughs present special challenges since the cold EM calorimeter is surrounded by warm hadronic calorimetry. Space allotted to feedthroughs either takes away space from the barrel hadronic calorimeter, or it increases the gap between the active regions of the barrel and endcap calorimeters. Minimizing the volume devoted to these feedthroughs (and their associated cabling) is clearly essential. These considerations force the feedthroughs to the outside corners of the barrel cryostat, a region which is also critical to ensuring the mechanical stability of the cryostat. An integrated solution is clearly required, which simultaneously considers the design of the cryostat itself along with the layout of the signal cables and feedthroughs in order to optimize the response of the overall ATLAS calorimetry in the transition region between the barrel and the endcap calorimeters.

Endcap Hadronic Calorimetry Although the ATLAS baseline design for the endcap hadronic modules is very similar to the Washington group's design for GEM, there are many details that must be studied and refined. One of the most challenging is the mating of large copper absorber plates with Kapton/Hexcell charge collection electrodes. This has been successfully achieved for a small test module, but the capability remains to be demonstrated for the $150 \mathrm{~cm}$ fullsize modules. High mechanical tolerances must be maintained to keep cracks between modules to a minimum (in the $1 \mathrm{~mm}$ range), and stress studies and tests must be conducted to ensure the mechanical robustness of the full size system.

Substantial work needs to be done to find the best way to bring signals out to the preamplifiers. The concerns here are electrical and mechanical performance under cryogenic conditions, and the very small space available as a result of hermeticity requirements. Several designs are under consideration, but further work, including prototype design, fabrication, and testing are needed.

There is currently no calorimeter test data for the ATLAS baseline design. Two beam tests are planned for FY1995 and FY1996 to verify the predicted physics performance of the system. Following the beam tests and validation of the engineering design, construction will begin in 1997 (preparations and tooling to begin in 1996).

We plan to share with several Canadian groups (centered at TRIUMF) responsibility for fabricating and testing one of the ATLAS hadronic endcaps. Module fabrication will include cold testing in all possible orientations to guarantee interchangeability of the modules. The Washington group has space sufficient for this task allocated in a new physics building (first occupation underway in fall 1994). This includes a clean room, and facilities for etching the Kapton electrodes and fabricating the two layer electrostatic transformers. A large assembly lab with crane coverage will be used for module assembly. Our involvement will continue through the installation and testing phases and the commissioning of the completed calorimeter.

Liquid Purity \& Radiation Damage Studies We have been pursuing two general goals: a) to verify that no component inside the cryostat releases any chemicals, as a consequence of radiation, that might affect the signals seen in the noble liquid; and b) to develop preamplifiers that will survive for a long time in a high radiation environment. In the first area, we must first complete the tests of the most essential components: the electrodes and 
cables. These will be irradiated and studied for any deleterious effects on charge collection in LAr and LKr. In the second area, we have pursued the development of JFET preamps for several years. We will continue the study of the effects of neutrons and of ionizing radiation on these JFET preamps over the next two years.

In addition, we will continue our measurements of positive ion buildup in noble liquid calorimeters in high rate environments. This has obvious implications on the design of such calorimeters in the very forward direction.

System Integration and Optimization The ATLAS cryogenic calorimeter is absolutely crucial to the success of the experiment. We are working with the ATLAS collaboration to optimize the overall performance of the experiment and to insure that the cryogenic barrel and endcap calorimetry is optimized relative to the other detector subsystems - e.g., we are working with the muon group to optimize the depth of the calorimeter in order to shield the muon system.

\subsection{Institutional Responsibilities}

The tasks we are taking on involve core responsibilities within the ATLAS noble liquid calorimeter subsystem. The US groups involved will carry out this work in close collaboration with other ATLAS groups that also have an interest in these areas. The individual groups specified below will have special responsibilities for the aspects of the work indicated, but the work will be distributed so that the overall effort is appropriately shared.

Readout Chain Architecture: BNL/Columbia

Level I Trigger: Pittsburgh/BNL

Preamps/Calibration: BNL

Driver: Arizona/Rochester/BNL

Shaper: BNL/Columbia

SCA/ADC/DSP: Columbia

Cables/Interconnects/Feedthroughs: BNL/Washington/Rochester

Endcap Hadronic Calorimeter: Washington

Barrel Cryostat: Rochester/BNL/SMU

System Integration \& Optimization: Columbia/BNL/Rochester/SMU

Radiation Damage Studies: Arizona/BNL

\subsection{Budget Summary}

The budget estimates provided in Table 2 follow the guidelines used previously to estimate the cost of similar components of the GEM detector. Thus the figures are inclusive of R\&D and EDIA, including engineering directly related to the development of the components for which we plan to take responsibility. They do not include the cost of salaries and benefits for physicists who will be working on the project. 


\subsection{Summary of Activities During FY1995 and FY1996}

During FY1995 we will carry out the following activities:

- Participate in RD3 test beam activities at CERN

- We plan to assist other ATLAS groups in the design and construction of a full size ATLAS EM prototype, and to participate in the 1995 and 1996 RD3 beam tests.

- Engineering studies of endcap hadronic calorimetry

- Together with our Canadian colleagues, we plan to design prototypes for the FY1995 test of the basic concept, initiate design and bench studies for the full scale test in FY1996, and to participate in these two beam tests.

- Establish an electronics certification center

- Assemble a 250 channel readout chain using existing components, and employ this system to test and optimize new readout components.

- Evaluate preamp and SCA prototypes

- Cold test new preamps.

- Test a new SCA and start design of a dual range digitizing board incorporating shapers, SCA's, and ADC's.

- Evaluate feedthrough prototypes

- Evaluate a 960 pin version of a potential ATLAS feedthrough based on the GEM design, and develop suitable connectors for proposed cables.

- Test a prototype version of an alternate, potentially higher density, continuous feedthrough.

- Study electronic readout and trigger architecture

- Continue work on defining and optimizing the calorimeter readout architecture.

- Engineering studies of the ATLAS barrel cryostat

- Carry out an in depth review of the preliminary ATLAS barrel cryostat design, and begin the process of adapting this design to US specifications and cost accounting.

- Radiation damage studies

- Expose those components of the calorimeter readout, such as preamps, that need to operate in a high radiation environment to both gamma and neutron radiation.

- EM calorimetry optimization and integration

- Analyze 1994 and 1995 RD3 test beam results in order to improve the design of the ATLAS barrel and endcap EM calorimeters and to investigate the role of the first section of the hadronic endcap in measuring very high energy electrons and photons. 
Table 3 summarizes by institution and funding agency the funding needed to carry out our proposed activities during FY1995. Table 4 summarizes by institution the non-physicist personnel needed to carry out this work.

Activities in FY1996 will build on what is accomplished during FY1995. The precise points of transition between fiscal years for each activity will depend very sensitively on both the magnitude and the timing of our FY1995 funding. Table 5 summarizes by institution and funding agency the funding needed to continue our proposed activities through FY1996, assuming that we receive in FY1995 funding at the level we propose. 
Table 2: Budget Estimates (Total Project Period)

\begin{tabular}{|l|c|c|c|}
\hline & Total Cost & DOE Cost & NSF Cost \\
\hline Calorimeter Readout & $\$ 45 \mathrm{M}$ & $\$ 17 \mathrm{M}$ & $\$ 13 \mathrm{M}$ \\
Kapton Electrodes & $\$ 15 \mathrm{M}$ & $\$ 6 \mathrm{M}$ & \\
Endcap Hadronic Calorimetry & $\$ 10 \mathrm{M}$ & & $\$ 4.6 \mathrm{M}$ \\
EM Feedthroughs & $\$ 3 \mathrm{M}$ & $\$ 3 \mathrm{M}$ & \\
Barrel Cryostat & $\$ 10 \mathrm{M}$ & & $\$ 10 \mathrm{M}$ \\
\hline \multicolumn{1}{|c|}{ Total US Cost } & & $\$ 26 \mathrm{M}$ & $\$ 27.6 \mathrm{M}$ \\
\hline
\end{tabular}

$\dagger$ Includes endcap feedthroughs

Table 3: FY1995 Proposed Institutional Budgets

\begin{tabular}{|c|c|c|c|c|}
\hline Institution & $\begin{array}{c}\text { Technical } \\
\text { Support }\end{array}$ & $\begin{array}{c}\text { Materials \& } \\
\text { Services }\end{array}$ & DOE Cost & NSF Cost \\
\hline Arizona $^{\dagger}$ & $*$ & $*$ & $*$ & \\
BNL $^{\dagger}$ & $\$ 400 \mathrm{~K}$ & $\$ 100 \mathrm{~K}$ & $\$ 500 \mathrm{~K}$ & \\
Columbia/Nevis & $\$ 70 \mathrm{~K}$ & $\$ 30 \mathrm{~K}$ & & $\$ 100 \mathrm{~K}$ \\
Pittsburgh $^{\dagger}$ & $\$ 80 \mathrm{~K}$ & $\$ 10 \mathrm{~K}$ & $\$ 90 \mathrm{~K}$ & \\
Rochester $^{\dagger}$ & $\$ 60 \mathrm{~K}$ & $\$ 15 \mathrm{~K}$ & $\$ 75 \mathrm{~K}$ & \\
Rochester$^{\text {SMU }}$ & $\$ 55 \mathrm{~K}$ & $\$ 20 \mathrm{~K}$ & & $\$ 75 \mathrm{~K}$ \\
Washington$\S$ & $\$ 15 \mathrm{~K}$ & $\$ 5 \mathrm{~K}$ & $\$ 20 \mathrm{~K}$ & \\
\hline FY1995 Totals & $\$ 740 \mathrm{~K}$ & $\$ 15 \mathrm{~K}$ & & $\$ 75 \mathrm{~K}$ \\
\hline
\end{tabular}

t DOE Support

$\S N S F$ Support

* Budget included as part of the forward calorimeter subsystem. 
Table 4: FY1995 Non-physicist Personnel by Institution

\begin{tabular}{|c|c|c|c|c|}
\hline Institution & FTE'S & Type & Cost & Comments \\
\hline Arizona & $*$ & $*$ & $*$ & forward cal \\
BNL & 2.5 & EEng & $\$ 290 \mathrm{~K}$ & readout \\
BNL & 1.0 & Designer & $\$ 60 \mathrm{~K}$ & system \\
BNL & 1.0 & Tech & $\$ 50 \mathrm{~K}$ & readout \\
Columbia/Nevis & 1.2 & EEng & $\$ 70 \mathrm{~K}$ & SCA's/ADC's \\
Pittsburgh & 1.5 & EEng & $\$ 80 \mathrm{~K}$ & analog sums \\
Rochester & 1.0 & MEng & $\$ 60 \mathrm{~K}$ & cryostat \\
Rochester & 0.2 & MEng & $\$ 15 \mathrm{~K}$ & feedthroughs \\
Rochester & 1.0 & Tech & $\$ 40 \mathrm{~K}$ & feedthroughs \\
SMU & 0.2 & Designer & $\$ 15 \mathrm{~K}$ & integration \\
Washington & 0.7 & MEng & $\$ 40 \mathrm{~K}$ & had endcap \\
Washington & 0.3 & MEng & $\$ 20 \mathrm{~K}$ & feedthroughs \\
\hline
\end{tabular}

* Personnel included as part of the forward calorimeter subsystem.

Table 5: FY1996 Proposed Institutional Budgets

\begin{tabular}{|c|c|c|c|c|}
\hline Institution & $\begin{array}{c}\text { Technical } \\
\text { Support }\end{array}$ & $\begin{array}{c}\text { Materials \& } \\
\text { Services }\end{array}$ & DOE Cost & NSF Cost \\
\hline Arizona $^{\dagger}$ & $*$ & $*$ & ${ }^{*}$ & \\
BNL $^{\dagger}$ & $\$ 750 \mathrm{~K}$ & $\$ 400 \mathrm{~K}$ & $\$ 1,150 \mathrm{~K}$ & \\
Columbia/Nevis & $\$ 170 \mathrm{~K}$ & $\$ 180 \mathrm{~K}$ & & $\$ 350 \mathrm{~K}$ \\
Pittsburgh $^{\dagger}$ & $\$ 110 \mathrm{~K}$ & $\$ 40 \mathrm{~K}$ & $\$ 150 \mathrm{~K}$ & \\
Rochester $^{\dagger}$ & $\$ 120 \mathrm{~K}$ & $\$ 35 \mathrm{~K}$ & $\$ 155 \mathrm{~K}$ & \\
Rochester$^{\text {SMU }}$ & $\$ 160 \mathrm{~K}$ & $\$ 15 \mathrm{~K}$ & & $\$ 175 \mathrm{~K}$ \\
Washington$^{\dagger}$ & $\$ 30 \mathrm{~K}$ & $\$ 10 \mathrm{~K}$ & $\$ 40 \mathrm{~K}$ & \\
\hline FY1996 Totals & $\$ 150 \mathrm{~K}$ & $\$ 25 \mathrm{~K}$ & & $\$ 175 \mathrm{~K}$ \\
\hline
\end{tabular}

† DOE Support

$\S N S F$ Support

* Budget included as part of the forward calorimeter subsystem. 
ow

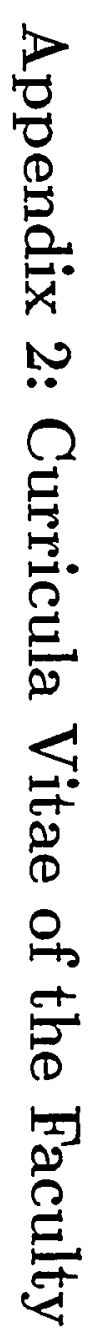




\title{
RYSZARD STROYNOWSKI Curriculum Vitae
}

Born: June 11, 1946 in Lodz, Poland.

\section{Citizenship: U.S.A.}

Married, two children.

\section{Education:}

1968 Magister degree (M.Sc.)

University of Warsaw, Poland.

1973 Doctorat en sciences physiques (Ph.D.)

University of Geneva, Switzerland.

\author{
Employment \\ 1993-present Professor, SMU. \\ 1991-1993 Associate Professor, SMU. \\ 1983-1991 Senior Research Associate, Caltech, Pasadena, CA 91125. \\ 1987 Lecturer, Caltech, Pasadena, CA 91125. \\ 1980-1983 Senior Research Fellow, Caltech, Pasadena, CA 91125. \\ 1975-1980 Research Associate, Group B, SLAC, Stanford, CA 94305. \\ 1970-1975 Staff Physicist, CERN, Geneva, Switzerland. \\ 1969-1970 Visiting Scientist, CERN, Geneva, Switzerland. \\ 1968-1969 Assistant with tenure, University of Warsaw, Poland.
}

\section{Other:}

1969 Niels Bohr Institute, Copenhagen, Denmark (7 weeks).

1972 University of Helsinki, Helsinki, Finland (8 weeks).

1983 Visiting Professor: Enseignement du troisieme cycle de la physique en Suisse Romande, Lausanne, Switzerland (one term).

1986 Visiting Professor, UCLA (one term).

1991-1993 Visiting Associate, Caltech, Pasadena, CA 94305.

1991-1993 Visiting Scientist, SSC Laboratory, Dallas, Texas. 


\section{LIST OF PUBLICATIONS 1989-1994}

I Publications in refereed journals

1. The Mark II detector for the SLC.

G. Abrams et al., Nuclear Instruments and Methods A281 (1989) 55.

2. Searches for non-minimal Higgs bosons from a virtual $\mathbf{Z}$ decaying into a muon pair at SLAC $e^{+} e^{-}$storage ring PEP.

S. Komamiya et al., Physical Review D40 (1989) 721.

3. Studies of jet production rates in $e^{+} e^{-}$annihilation at $E_{c m}=29 \mathrm{GeV}$.

S. Bethke et al., Zeitschrift der Physik C43 (1989) 325.

4. Initial measurements of $\mathrm{Z}$ boson resonance parameters in $e^{+} e^{-}$annihilation.

G. Abrams et al., Physical Review Letters 63 (1989) 724.

5. Search for B decay to Higgs bosons for Higgs boson masses between 50 $\mathrm{MeV} / \mathrm{c}^{2}$ and $210 \mathrm{MeV} / \mathrm{c}^{2}$.

A. Snyder et al., Physics Letters B229 (1989) 169.

6. First measurements of hadronic decays of the $\mathrm{Z}$ boson.

G. S. Abrams et al., Physical Review Letters 63 (1989) 1558.

7. Measurements of $\mathbf{Z}$ boson resonance parameters in $e^{+} e^{-}$annihilation. G. S. Abrams et al., Physical Review Letters 63 (1989) 2173.

8. Searches for new quarks and leptons produced in $\mathrm{Z}$ boson decay. G. S. Abrams et al., Physical Review Letters 63 (1989) 2447.

9. Measurement of $Z$ decays into lepton pairs.

G. S. Abrams et al., Physical Review Letters 63 (1989) 2780.

10. Determination of $\alpha_{s}$ using differential jet multiplicity distribution at PEP and SLC.

S. Komamiya et al., Physical Review Letters 64 (1990) 987.

11. Search for long-lived massive neutrinos in $\mathrm{Z}$ decays. C. K. Jung et al., Physical Review Letters 64 (1990) 1091.

12. Upper limit on $D^{ \pm}$and $B^{ \pm}$decays to two leptons plus $\pi^{ \pm}$or $K^{ \pm}$. A. Weir et al., Physical Review D41 (1990) 1384.

13. Measurement of the $B^{0}$ meson lifetime.

S. R. Wagner et al., Physical Review Letters 64 (1990) 1095.

14. Measurement of the $b \bar{b}$ fraction in hadronic $Z$ decays.

F. Kral et al., Physical Review Letters 64 (1990) 1221. 
15. Measurements of charged particle inclusive distributions in hadronic decays of the $\mathrm{Z}$ boson.

G. S. Abrams et al., Physical Review Letters 64 (1990) 1334.

16. Decay time and light yield measurements for plastic scintillating fibers. C. M. Hawkes, M. Kuhlen, B. Milliken, R. Stroynowski and E. Wicklund, Nuclear Instruments and Methods A292 (1990) 329.

17. A reanalysis of $B^{0}-\bar{B}^{0}$ mixing in $e^{+} e^{-}$annihilations at $29 \mathrm{GeV}$. A. Weir et al., Physics Letters B240 (1990) 289.

18. A search for decays of the $Z$ to unstable neutral leptons with mass between 2.5 and $22 \mathrm{GeV}$.

P. R. Burchat et al., Physical Review D41 (1990) 3542.

19. A search for doubly charged Higgs scalars in $Z$ decays. M. L. Swartz et al., Physical Review Letters 64 (1990) 2877.

20. Search for non-minimal neutral Higgs bosons from $\mathbf{Z}$ boson decays. S. Komamiya et al., Physical Review Letters 64 (1990) 2881.

21. A search for pair production of heavy stable charged particles in $\mathbf{Z}$ decays. E. Soderstrom et al., Physical Review Letters 64 (1990) 2980.

22. Searches for supersymmetric particles produced in $\mathbf{Z}$ boson decay. T. Barklow et al., Physical Review Letters 64 (1990) 2984.

23. Measurement of the total hadronic cross-section in $e^{+} e^{-}$annihilation at $s^{* *}(1 / 2)=29 \mathrm{GeV}$.

C. von Zanthier et al., Physical Review D43 (1991) 34.

24. A search for elastic nondiagonal lepton pair production in $e^{+} e^{-}$annihilation at $s^{* *}(1 / 2)=29 \mathrm{GeV}$.

J. J. Gomez-Cadenas et al., Physical Review Letters 66 (1991) 1007.

25. Timing properties of long scintillation counters based on scintillating fibers.

M. Kuhlen, M. Moszynski, R. Stroynowski, E. Wicklund and B. Milliken, Nuclear Instruments and Methods A301 (1991) 223.

26. Measurement of the inclusive $B^{*}$ cross section above $\Upsilon(4 \mathrm{~S})$. D. S. Akerib et al., Physical Review Letters 67 (1991) 1692.

27. Inclusive $\chi(2 \mathrm{P})$ production in $\Upsilon(3 \mathrm{~S})$ decay. R. Morrison et al., Physical Review Letters 67 (1991) 1696.

28. Measurement of the $b \bar{b}$ fraction in hadronic $Z^{0}$ decays with precision vertex detectors.

R. G. Jacobsen et al., Physical Review Letters 67 (1991) 3347. 
29. Comparison of the timing properties of the new Philips Components (Amperex) XP2020/UR photomultiplier with the XP2020 photomultiplier. M. Kuhlen, E. Wicklund, R. Stroynowski and B. Milliken, IEEE Transactions on Nuclear Science 38 (1991) 1052.

30. Two-Body $D_{s}^{+}$decays to $\eta \pi^{+}, \eta^{\prime} \pi^{+}, \eta \rho^{+}, \eta^{\prime} \rho^{+}$, and $\phi \rho^{+}$. M. Daoudi et al., Physical Review D45 (1992) 3965.

31. Electronic branching ratio of the tau lepton. R. Ammar et al., Physical Review D45 (1992) 3976.

32. Measurement of the charged multiplicity of events containing bottom hadrons at $\mathrm{E}(\mathrm{cm})=91 \mathrm{GeV}$.

B. A. Schumm et al., Physical Review D46 (1992) 453.

33. Observation of the Decay $\Xi_{c}^{0} \rightarrow \Omega^{-} K^{+}$.

S. Henderson et al., Physics Letters B283 (1992) 161.

34. $D_{s}^{+}$decays to $\eta \rho^{+}, \eta \prime \rho^{+}$and $\phi \rho^{+}$.

P. Avery et al., Physical Review Letters 68 (1992) 1279.

35. $D_{s}^{+}$decays to $\eta \pi^{+}$and $\eta / \pi^{+}$.

J. Alexander et al., Physical Review Letters 68 (1992) 1275.

36. The CLEO II detector.

Y. Kubota et al., Nuclear Instruments and Methods A320 (1992) 66.

37. Isospin mass splittings from precision measurements of $D^{*}-D$ mass differences.

D. Bortoletto et al., Physical Review Letters 69 (1992) 2046.

38. Measurement of the $D^{*}(2010)$ branching fractions.

F. Butler et al., Physical Review Letters 69 (1992) 2041.

39. Measurement of $\tau$ decays involving $\eta$ mesons.

M. Artuso et al., Physical Review Letters 69 (1992) 3278.

40. Measurement of the tau lepton electronic branching fraction.

D.S. Akerib et al., Physical Review Letters 69 (1992) 3610.

41. A measurement of the tau lepton lifetime.

M. Battle et al., Physics Letters B291 (1992) 488.

42. A search for $\tau^{-} \rightarrow \mu^{-}+\gamma$ : a test of lepton number conservation.

A. Bean et al., Physical Review Letters 70 (1993) 138.

43. Exclusive $\chi(2 P)$ production in $\Upsilon(3 \mathrm{~S})$ decay.

G. Crawford et al., Physics Letters B294 (1992) 139.

44. Shape studies of quark jets vs gluon jets at $\sqrt{=10 \mathrm{GeV}}$. M. S. Alam et al., Physical Review D46 (1992) 4822. 
45. Tau decays with one charged particle plus multiple $\pi^{0}$ s. M. Procario et al., Physical Review Letters 70 (1993) 1207.

46. Lepton asymmetry measurements in $\bar{B} \rightarrow D^{*} l^{-} \overline{\nu_{l}}$ and implications for V-A and the form factors.

S. Sanghera et al., Physical Review D47 (1993) 791.

47. Search for exclusive $b \rightarrow u$ semileptonic decays of B mesons.

A. Bean et al., Physical Review Letters 70 (1993) 2681.

48. A measurement of the tau lepton mass.

R. Balest et al., Physical Review D47 (1993) 3671.

49. A limit on the tau neutrino mass.

D. Cinabro et al., Physical Review Letters 70 (1993) 3700.

50. Evidence for penguin-diagram: First observation of $B \rightarrow K^{*}(892) \gamma$. R. Ammar et al., Physical Review Letters 71 (1993) 674.

51. Production and decay of the $D_{s 1}(2536)^{+}$.

J. P. Alexander et al., Physics Letters B 303 (1993) 377.

52. Measurement of the ratio $B\left(D^{+} \rightarrow \pi^{0} l^{+} \nu\right) / B\left(D^{+} \rightarrow \overline{K^{0}} l^{+} \nu\right)$.

M. S. Alam et al., Physical Review Letters 71 (1993) 1311.

53. Two measurements of $B^{0} \bar{B}^{0}$ mixing.

J. Bartelt et al., Physical Review Letters 71 (1993) 1680.

54. Measurement of the decay $\tau^{-} \rightarrow \pi^{-} \pi^{+} \pi^{-} 2 \pi^{0} \nu_{\tau}$.

D. Bortoletto et al., Physical Review Letters 71 (1993) 1791.

55. Measurement of the $D \rightarrow \pi \pi$ branching fractions.

M. Selen et al., Physical Review Letters 71 (1993) 1973.

56. Study of the decays $\Lambda_{c}^{+} \rightarrow \Xi^{0} K^{+}, \Lambda_{c}^{+} \rightarrow \Sigma^{+} K^{+} K^{-}$and $\Lambda_{c}^{+} \rightarrow \Xi^{-} K^{+} \pi^{+}$.

P. Avery et al., Physical Review Letters 71 (1993) 2391.

57. Measurement of the absolute branching fraction for $D^{0} \rightarrow K^{-} \pi^{+}$.

D. S. Akerib et al., Physical Review Letters 71 (1993) 3070.

58. Observation of the charmed baryon $\Sigma_{c}^{+}$and measurement of the isospin mass splittings of the $\Sigma_{c}$.

G. Crawford et al., Physical Review Letters 71 (1993) 3259.

59. Measurement of exclusive $\Lambda_{c}$ decays with a $\Sigma^{+}$in the final state.

Y. Kubota et al., Physical Review Letters 71 (1993) 3255.

60. Study of $D^{0}$ decays into $\bar{K}^{0}$ and $\overline{K^{* 0}}$.

M. Procario et al., Physical Review D 48 (1993) 4007.

61. Observation of $B^{0}$ decay to two charmless mesons.

M. Battle et al., Physical Review Letters 71 (1993) 3922. 
62. The tau lepton and its neutrino.

R. Stroynowski and A. J. Weinstein,

Annual Review of Nuclear and Particle Physics 1993 43: 457-528.

63. Measurement of charmless semileptonic decays of $\mathrm{B}$ mesons.

J. Bartelt et al., Physical Review Letters 71 (1993) 4111.

64. Measurement of exclusive semileptonic decays od $D$ mesons.

A. Bean et al., Physics Letters B 317 (1993) 647.

65. Search for exclusive $b \rightarrow u$ transitions in hadronic decays of $\mathrm{B}$ mesons involving $D_{s}^{+}$and $D_{s}^{*+}$ mesons.

J. P. Alexander et al., Physics Letters B 319 (1993) 365.

66. Observation of $D^{0} \rightarrow K^{+} \pi^{-}$.

D. Cinabro et al., Physical Review Letters 72 (1994) 1406.

67. Observation of a new charmed strange meson.

Y. Kubota et al., Physical Review Letters 72 (1994) 1972.

68. Measurement of the branching fraction for $D^{+} \rightarrow K^{-} \pi^{+} \pi^{+}$.

R. Balest et al., Physical Review Letters 72 (1994) 2328.

69. Analysis of hadronic transitions in $\Upsilon(3 S)$ decays.

F. Butler et al., Physical Review D 49 (1994) 40.

70. Study of the decay $\Lambda_{c}^{+} \rightarrow \Lambda l^{+} \nu_{l}$.

T. Bergfeld et al., Physics Letters B 323 (1994) 219.

71. First measurement of $\Gamma\left(D_{s}^{+} \rightarrow \mu^{+} \nu\right) / \Gamma\left(D_{s}^{+} \rightarrow \phi \pi^{+}\right)$.

D. Acosta et al., Physical Review D 49 (1994) 5690.

72. Search for $B^{0}$ decays to two charged leptons.

R. Ammar et al., Physical Review D 49 (1994) 5701.

73. Measurement of the branching fraction $B\left(\tau^{ \pm} \rightarrow h^{-} \pi^{0} \nu_{\tau}\right)$.

M. Artuso et al., Physical Review Letters 72 (1994) 3762.

74. Exclusive hadronic $B$ decays to charm and charmonium final states.

M. S. Alam et al., Physical Review D 50 (1994) 43.

75. Luminosity measurement with the CLEO II detector.

G. Crawford et al., Nuclear Instruments and Methods in Physics research A 345 (1994) 429.

76. A measurement of $B\left(D_{s}^{+} \rightarrow \phi l^{+} \nu\right) / B\left(D_{s}^{+} \rightarrow \phi \pi^{+}\right)$.

F. Butler et al., Physics Letters B 324 (1994) 255.

77. Study of the five-charged-pion decays of the $\tau$ lepton. D. Gibaut et al., Physical Review Letters 73 (1994) 934.

78. Measurement of Cabibbo-suppressed decays of the $\tau$ lepton.

M. Battle et al., Physical Review Letters 73 (1994) 1079. 
79. Production and decay of $D_{1}(2420)^{0}$ and $D_{2}^{*}(2460)^{0}$. P. Avery et al., Physics Letters B 331 (1994) 236.

80. Observation of $\Lambda_{c}^{+}$decays to $\Lambda \pi^{+} \pi^{0}, \Sigma^{0} \pi^{+}, \Sigma^{0} \pi^{+} \pi^{0}$, and $\Sigma^{0} \pi^{-} \pi^{+} \pi^{+}$. P. Avery et al., Physics Letters B 325 (1994) 257.

II Conference papers

81. Time of Flight Technique. R. Stroynowski, Proceedings of the Tau-Charm Factory Workshop, Stanford Linear Accelerator Center, May 1989.

CALT-68-1565. SLAC-Report 343, page 956 (1989).

82. Supersymmetry and $\tau$ decays.

R. Stroynowski,

Proceedings of the Tau-Charm Factory Workshop, Stanford Linear Accelerator Center, May 1989 .

CALT-68-1572. SLAC-Report 343, page 589 (1989).

83. Search for supersymmetry in $\tau$ decays.

R. Stroynowski,

Contribution to the Workshop on Electroweak Interactions, Ginosar, Israel, March 1989. Nuclear Physics B (Proc.Suppl.) 13 (1990) 176.

84. Present issues in tau physics.

R. Stroynowski, Proceedings of the XIII Warsaw Symposium on Elementary Particle Physics. Kazimierz, Poland, June 1990. "Physics of Elementary Interactions", World Scientific (1991) Z. Ajduk, S. Pokorski and A. K. Wroblewski editors.

85. Light propagation and timing with scintillating fibers.

R. Stroynowski,

Contribution to the 2nd International Conference on Advanced Technology and Particle Physics, Como, Italy 11-15 June, 1990.

Nuclear Physics B (Proc. Suppl.) 23A (1991) 273.

86. Leptonic decays of the tau.

R. Stroynowski, Proceedings of the Workshop on Tau Lepton Physics, Orsay, France, September 24-27 1990. Editions Frontiere (1991). Edited by M. Davier and B. Jean-Marie.

87. Tau decays at CLEO.

R. Stroynowski,

Proceedings of the Joint International Lepton-Photon Symposium and Europhysics 
Conference on High Energy Physics, Geneva, July 1991. World Scientific (1992). Edited by S. Heagarty, K. Potter and E. Quercigh.

88. Design concept for the GEM detector magnet.

B. A. Smith et al.,

Applied Superconductivity Conference, Chicago, August 23-28, 1992.

89. Overview of the superconducting magnet subsystem for the GEM detector at the SSC.

G. Deis et al.,

"Supercollider 5". 5th IISSC Symposium, San Francisco, Ca., May 1993.

90. Conductor design for the GEM detector magnet.

J. V. Minervini et al.,

"Supercollider 5". 5th IISSC Symposium, San Francisco, Ca., May 1993.

91. Forbidden decays of $\tau$ lepton from CLEO.

R. Stroynowski, Representing CLEO Collaboration.

Proceedings of the International EPS Concerence on High Energy Physics, Marseille, France, July 22-28, 1993, page 143.

Editions Frontieres, J. Carr and M. Perrottet editiors.

92. Exclusive and rare $\tau$ lepton decays.

R. Stroynowski

Proceedings of the International EPS Concerence on High Energy Physics, Marseille, France, July 22-28, 1993, page 484.

Editions Frontieres, J. Carr and M. Perrottet editiors.

93. The superconducting solenoid magnet system for the GEM detector at the SSC.

G. Deis et al.

13th International Conference on Magnet Technology, Victoria, Canada, September 20-24, 1993.

\section{Technical notes}

94. Tau-Charm Factory Design.

B. Barish et al., SLAC-PUB-5180 (1990).

95. An Expression of Interest to Construct a Major SSC Detector.

R. V. Steiner et al., The GEM Collaboration.

SSC EOI-0020, July 8, 1991.

96. GEM Baseline design.

GEM Collaboration, GEM-TN-92-76, (1992). 
97. GEM Magnet Engineering Design Report.

R. Stroynowski et al., The GEM Magnet Design Team,

GEM-TN-92-116 (1992).

98. GEM Magnet Subsystem Specification.

L. Parlier et al., The GEM Magnet Design Team,

GEM TN-92-234 (1992).

99. GEM Technical Design Report.

W. C. Lefmann et al., The GEM Collaboration.

SSCL-SR-1219, GEM-TN-93-262 (1993).

100. The CLEO III Detector: Design and Physics Goals.

The CLEO Collaboration. CLNS94/1277 (1994).

IV Papers submitted for publication or in preparation

101. Measurement of Cross section for $\gamma \gamma \rightarrow p \bar{p}$.

M. Artuso et al., The CLEO Collaboration.

CLNS 93/1245, CLEO 93-17 (1993). Submitted for publication.

102. Measurement of two-photon production of the $\chi_{c 2}$.

V. Shelkov et al., The CLEO Collaboration.

CLNS 93/1250, CLEO 93-19 (1993). Submitted for publication.

103. Observation of inclusive $B$ decays to the charmed baryons $\Sigma_{c}^{++}$and $\Sigma_{c}^{0}$. M.Procario et al., The CLEO Collaboration.

CLNS 93/1264, CLEO 93-24 (1993). Submitted for publication.

104. Precision measurement of the $D_{s}^{+*}-D_{s}^{+}$mass difference.

D. N. Brown et al., The CLEO Collaboration.

CLNS 94/1271, CLEO 94-6 (1994). Submitted for publication.

105. Two-photon production of charged pion and kaon pairs.

J. Dominick et al., The CLEO Collaboration.

CLNS 94/1274, CLEO 94-9 (1994). Submitted for publication.

106. Measurement of the $\bar{B} \rightarrow D^{*} l \bar{\nu}$ branching fractions and $\left|V_{c b}\right|$.

B. Barish et al., The CLEO Collaboration.

CLNS 94/1285, CLEO 94-13 (1994). Submitted for publication.

107. Search for neutrinoless decays of the tau lepton.

J. Bartelt et al., The CLEO Collaboration.

CLNS 94/1287, CLEO 94-15 (1994). Submitted for publication.

108. Semileptonic branching fractions of charged and neutral B mesons. M. Athanas et al., The CLEO Collaboration.

CLNS 94/1286, CLEO 94-16 (994). Submitted for publication. 
109. Axion search with optical technique.

Ryszard Stroynowski and Ariel Zhitnitsky.

SMU-HEP 94-12 (1994). Submitted for publication. 


\section{CURRICULUM VITAE}

Name: $\quad$ Tomasz Skwarnicki

Assistant Professor of Physics

Address:

Mail:

Department of Physics

Southern Methodist University

106 Fondren Science Bldg.

Dallas, TX 75275-0175

Telephone: (214) $768-2819$

Telefax:

(214) $768-4095$

E-Mail:

Education: $\quad$ Ph.D. Physics

tomasz@mail.physics.smu.edu

Employment History :

M.Sc. Physics

Institute of Nuclear Physics, Krakow, Poland (1986).

Jagiellonian University, Krakow, Poland (1982).

$8 / 92-$

$5 / 92-8 / 92$

Assistant Professor, Southern Methodist University, Dallas,

$8 / 89-5 / 92$

Guest Scientist, Superconducting Supper Collider, Dallas, I

$1 / 88-8 / 89$

Assistant Professor, Syracuse University, Syracuse, NY.

$8 / 85-12 / 87$

Research Associate, Syracuse University, Syracuse, NY.

Research Associate, DESY, Hamburg, Germany.

\section{Professional record includes}

- Co-author of over 100 papers on experimental high energy physics. Presented 17 talks at conferences and workshops and 22 seminars and colloquia.

- Was awarded Outstanding Junior Investigator Grant by the DoE and SSC fellowship by the TNRLC.

- Involved in four experiments (LENA, Crystal Ball, CLEO, CLEO-II) at two $e^{+} e^{-}$storage rings (DORIS, CESR). Participated in the GEM collaboration (SSC).

- Recognized expert in $\tau$ physics. Coordinated for a year work of over 30 physicist from 6 institutions working on tau physics in the CLEO collaboration. Analyzed $\tau$ decays to multi- $\pi^{\circ}$ final states and channels with $\eta$ mesons in Crystal Ball and CLEO-II. The first observations of $\tau \rightarrow \nu \pi 2 \pi^{\circ}, \tau \rightarrow \nu \pi 3 \pi^{\circ}$ and $\tau \rightarrow \nu \pi \pi^{\circ} \eta$. Supervised students working on precise measurement of branching fractions for $\tau \rightarrow \nu 3 \pi$ and $\tau \rightarrow \nu 3 \pi \pi^{\circ}$ and in search for flavor violating decays. Co-authored 17 publications on $\tau$ physics. Spoke on $\tau$ physics at 6 conferences/workshops and 9 seminars/colloquia. Organized parallel session on tau physics at the DPF conference in Albuquerque in August 1994 (also gave a summary talk of the session). Reviewed tau physics at the APS conference in Crystal City in April 1994. Presented CLEOII $\tau$ physics program to the HEPAP subpanel meeting, Ithaca, 1990. Mini-review talk at the Europhysics Conference in Uppsala, 1987.

- Recognized expert on $\Upsilon$ spectroscopy. Award by the Counsel of Atomic Research in Poland for thesis research on radiative transitions from $\Upsilon(2 S)$ studied with the Crystal Ball. Produced the first CLEO-II conference results and the first publication analyzing radiative transitions from $\Upsilon(3 S)$. Studied potential impact of a $B$-factory on $\Upsilon$ spectroscopy (part of the Cornell $B$-factory proposal). Co-authored 17 publications on $\Upsilon$ spectroscopy. Spoke on $\Upsilon$ spectroscopy at 5 conferences/workshops and 7 seminars/colloquia. Wrote a review article on $\Upsilon$ spectroscopy published in the 1993 edition of the Annual Review of Nuclear and Particle Science. 
- Involved in $B$-physics program in Crystal Ball and CLEO-II. Produced the first measurement of the inclusive $b \rightarrow s \gamma$ rate (CLEO) which was one of the highlights of the Glasgow conference, July 1994. Analysis of the electron endpoint spectrum for $V_{u b}$ determination and study of $B \rightarrow \nu e D^{*}$ (Crystal Ball). Co-authored 25 publications on $B$ meson decays. Spoke on $B$ physics at 3 conferences/workshops and 4 seminars.

- First observation of $\gamma \gamma \rightarrow \pi_{2}$ with the Crystal Ball detector.

- Two out of the three new detector components in CLEO-II (muons system, CsI(TI) crystals) are analyzed by event reconstruction programs developed by him.

- Played a major role in the assembly and in the cosmic ray testing of muon chambers constructed at Syracuse University for the CLEO-II detector. Co-author of the publication on the CLEO-II muon system.

- Contributed to the generic R\&D on a fast scintillation calorimeter based on pure CsI crystals (with Woody et al.). Co-author of the publication on this subject.

- Involved in design of the GEM detector for the SSC. Guest scientist at SSC for summer 1992. Coordinated the group developing physics simulation software for GEM. The developed simulation package was used in physics studies included in the GEM Technical Design Report. Presented the above simulation work to the PAC in May, 1993.

- Studied applications of commercial 3D graphic packages to high energy physics (collaboration with Northeast Parallel Architectures Center at Syracuse University).

- Taught 7 different courses at Syracuse University and Southern Methodist University. Wrote a 150 page long laboratory manual for the undergraduate class at Syracuse University. Advising 6 graduate students at SMU who work on the CLEO-II data.

\section{LIST OF PUBLICATIONS for the last 5 years}

Invited talks at National and International Conferences on High Energy Physics:

1. "First Measurement of Inclusive $b \rightarrow s \gamma$ Rate", T. Skwarnicki for the CLEO collaboration, Talk presented at the Joint Fall Meeting of the Texas Section of the APS, AAPT, and Zone 13 of the SPS, University of Texas at Austin, Austin, TX, 14-15 October, 1994.

2. "Summary of Tau Physics Results", T. Skwarnicki, Summary of parallel session presented at the $8^{\text {th }}$ Meeting of the Division of Particles and Fields of the American Physical Society, Albuquerque, New Mexico, 2-6 August, 1994.

3. "Status of Tau Physics", T. Skwarnicki, Review talk presented at the Joint Meeting of the American Physical Society and the American Association of Physics Teachers, Crystal City, VA, 18-22 April, 1994.

4. "CLEO-II Results on $\tau$ Decays to $\eta$ ", T. Skwarnicki for the CLEO collaboration, Talk presented at the Second Workshop on Tau Lepton Physics, The Ohio State University, Columbus, OH, Sept. 8-11, 1992, K.K. Gan (Ed.) World Scientific, 1993, p. 217.

5. "Performance of the CLEO-II CsI(TI) Calorimeter", T. Skwarnicki, Talk presented at " $\mathrm{B}$ factories, The State of the Art in Accelerators, Detectors and Physics", Stanford Linear Accelerator Center, Stanford, CA, April 6-10, 1992, D. Hitlin (Ed.) SLAC400, CONF-9204126, p. 526.

6. " $\Upsilon$ Spectroscopy at $B$ Factory", T. Skwarnicki, Talk presented at the TRIUMF-IPP $B$ Factory Workshop, Vancouver, B.C. Canada, 14-15 February 1991, CLNS 91-1049, HEPSY 91-1, Feb. 1991.

7. "Preliminary CLEO-II Results on Radiative Transitions from the $\Upsilon(3 S)$ ", T. Skwarnicki for the CLEO Collaboration,

Talk presented at the 25th International Conference on High Energy Physics, Singapore, 2-8 
August 1990, Parallel Session on Hadron Spectroscopy and Photon-photon Collisions; Contributed Paper \#748; published in the proceedings, K.K.Phua, Y.Yamaguchi (Eds.), South East Asia Theoretical Physic s Association and Physical Society of Japan, 1991, p. 550.

8. "Initial Performance of the CLEO-II CsI Calorimeter", T. Skwarnicki for the CLEO Collaboration, Talk presented at the 25th International Conference on High Energy Physics, Singapore, 2-8 August 1990, Parallel Session on Detector R\&D; Contributed Paper \#747; published in the proceedings, K.K.Phua, Y.Yamaguchi (Eds.), South East Asia Theoretical Physics Association and Physical Society of Japan, 1991, p. 1359.

9. " $\tau$ and $\Upsilon$ Physics with CLEO-II", T. Skwarnicki for the CLEO Collaboration, Talk presented to the HEPAP subpanel meeting at Ithaca, February 8, 1990.

10. "Radiative Transitions in the $\Upsilon$ System (Future Prospects)", T. Skwarnicki, Talk presented at the Workshop Towards Establishing a b-Factory, Syracuse University, Syracuse, New York, September 6-9, 1989, published in the proceedings, M. Goldberg, S. Stone (Ed.), Syracuse University 1989, p. 1.113.

11. "Tau Decays To Multiphoton Final States", T. Skwarnicki, Talk presented at the Tau-Charm Factory Workshop, SLAC, Stanford, California, May 23-27, 1989, published in the proceedings, L.V. Beers (Ed.), SLAC-Report-343, p. 519.

12. "CLEO Results on Charmed Hadron Spectroscopy", T. Skwarnicki for the CLEO Collaboration,

Talk presented at the 24th Recontres de Moriond "New Results in Hadronic Interactions", Les Arcs, France, March 12-18, 1989, published in the proceedings, J. Tran Thanh Van (Ed.), Gif-sur-Yvette : Editions Frontieres, 1989, p. 575.

\section{Journal Publications}

\section{CLEO EXPERIMENT}

1. "Study of the five-charged-pion decays of the $r$ lepton", with D. Gibaut et al.Phys. Rev. Lett. 73, 934 (1994).

2. "Production and decay of $D_{1}(2420)^{0}$ and $D_{2}^{*}(2460)^{0}$ " with P. Avery et al.Phys. Lett. B331, 236 (1994).

3. "Observation of $\Lambda_{c}^{+}$Decays to $\Lambda \pi^{+} \pi^{0}, \Sigma^{0} \pi^{+}, \Sigma^{0} \pi^{+} \pi^{0}$, and $\Sigma^{0} \pi^{-} \pi^{+} \pi^{+}$", with P. Avery et al.Phys. Lett. B325, 257 (1994).

4. "Measurement of Cabibbo-suppressed Decays of the $\tau$ Lepton", with M. Battle et al.Phys. Rev. Lett. 73, 1079 (1994).

5. "Exclusive Hadronic $B$ Decays to Charm and Charmonium Final States", with M.S. Alam et al.Phys. Rev. D50, 43 (1994).

6. "Search for $B^{0}$ Decays to Two Charged Leptons", R. Ammar et al.Phys. Rev. D49, 5701 (1994).

7. "First Measurement of $\Gamma\left(D_{s}^{+} \rightarrow \mu^{+} \nu\right) / \Gamma\left(D_{s}^{+} \rightarrow \phi \pi^{+}\right)$" with D. Acosta et al.Phys. Rev. D49, 5690 (1994).

8. "A Measurement of $\mathcal{B}\left(D_{s}^{+} \rightarrow \phi \ell^{+} \nu_{\ell}\right) / \mathcal{B}\left(D_{s}^{+} \rightarrow \phi \pi^{+}\right)$", with F. Butler et al.Phys. Lett. B324, 255 (1994).

9. "Measurement of the Branching Fraction for $D^{+} \rightarrow K^{-} \pi^{+} \pi^{+}$", with R. Balest et al.Phys. Rev. Lett. 72, 2328 (1994).

10. "Observation of a New Charmed Strange Meson", with Y. Kubota et al.Phys. Rev. Lett. 72,1972 (1994).

11. "Measurement of the Branching Fraction $\mathcal{B}\left(\tau^{-} \rightarrow h^{-} \pi^{0} \nu_{\tau}\right)$ " with M. Artuso et al.Phys. Rev. Lett. 72, 24 (1994). 
12. "Luminosity Measurement with the CLEO II Detector", with G. Crawford et al.Nucl. Inst.\& Methods A345, 429 (1994).

13. "Analysis of Hadronic Transitions in $\Upsilon(3 S)$ Decays", with F. Butler et al.Phys. Rev. D49 40 (1994).

14. "Search for Exclusive $b \rightarrow u$ Transitions in Hadronic Decays of $B$ Mesons Involving $D_{s}^{+}$and $D_{s}^{*+}$ Mesons", with J.P. Alexander et al.Phys. Lett. B319 365 (1993).

15. "Measurement of Charmless Semileptonic Decays of $B$ Mesons", with J. Bartelt et al.Phys. Rev. Lett. 714111 (1993).

16. "Observation of $B^{0}$ decay to Two Charmless Mesons", with M. Battle et al.Phys. Rev. Lett. 713922 (1993).

17. "Measurements of Exclusive Semileptonic Decays of $D$ Mesons", with A. Bean et al.Phys. Lett. B317 647 (1993).

18. "Observation of the Charmed Baryon $\Sigma_{c}^{+}$and Measurement of the Isospin Mass Splittings of the $\Sigma_{c}$ ", with G. Crawford et al.Phys. Rev. Lett. 713259 (1993).

19. "Measurement of Exclusive $\Lambda_{c}$ Decays with a $\Sigma^{+}$in the Final State", with Y. Kubota et al.Phys. Rev. Lett. 713255 (1993).

20. "Measurement of the Absolute Branching Fraction for $D^{0} \rightarrow K^{-} \pi^{+}$", with D.S. Akerib et al.Phys. Rev. Lett. 713070 (1993).

21. "Study of $D^{0}$ decays into $\bar{K}^{0}$ and $\bar{K}^{* 0 "}$ ", with M. Procario et al.Phys. Rev. D48 4007 (1993).

22. "Study of the Decays $\Lambda_{c}^{+} \rightarrow \Xi^{0} K^{+}, \Lambda_{c}^{+} \rightarrow \Sigma^{+} K^{+} K^{-}$and $\Lambda_{c}^{+} \rightarrow \Xi^{-} K^{+} \pi^{+}$", P. Avery et al.Phys. Rev. Lett. 712391 (1993).

23. "Measurement of the $D \rightarrow \pi \pi$ Branching Fractions", with M. Selen et al.Phys. Rev. Lett. 711973 (1993).

24. "Measurement of the Ratio $\mathcal{B}\left(D^{+} \rightarrow \pi^{0} \ell^{+} \nu\right) / \mathcal{B}\left(D^{+} \rightarrow \bar{K}^{0} \ell^{+} \nu\right)$ ", with M.S. Alam et al.Phys. Rev. Lett. 711311 (1993)

25. "A limit on the tau neutrino mass", with D. Cinabro et al.Phys. Rev. Lett. 70, 3700 (1993).

26. "Two measurements of $B^{0} \bar{B}^{0}$ mixing", with J. Bartelt et al.Phys. Rev. Lett. 71, 1680 (1993).

27. "Measurement of the decay $\tau^{-} \rightarrow \pi^{-} \pi^{+} \pi^{-} 2 \pi^{\circ}$ ", with D. Bortolleto et al.Phys. Rev. Lett. 71, 1791 (1993).

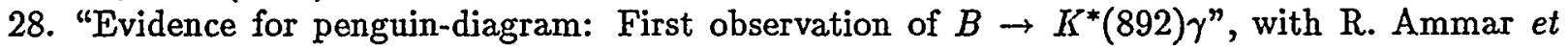
al.Phys. Rev. Lett. 71, 674 (1993).

29. "Measurement of the $\tau$-lepton mass", with R. Balest et al.Phys. Rev. D47, Rapid Communications, R3671 (1993).

30. "Search for Exclusive $b \rightarrow u$ Semileptonic Decays of $B$ Mesons", with A. Bean et al.Phys. Rev. Lett. 70, 2681 (1993).

31. "Production and Decay of the $D_{s 1}(2536)^{+}$", with J.P. Alexander et al.Phys. Lett. B303, 377 (1993).

32. "Lepton Asymmetry Measurements in $\bar{B} \rightarrow D^{*} l \nu_{l}$ and Implications for V-A and the Form Factors", with S. Sanghera et al.Phys. Rev. D47, 791 (1993).

33. "Tau Decays with One Charged Particle plus Multiple $\pi^{0}$ 's", with M. Procario et al.Phys. Rev. Lett. 70, 1207 (1993).

34. "A Search for $r^{-} \rightarrow \gamma \mu^{-}:$A Test of the Lepton Number Conservation", with A. Bean et al.Phys. Rev. Lett. 70, 138 (1993).

35. "Measurement of the Tau Lepton Electronic Branching Fraction", with D.S. Akerib et al.Phys. Rev. Lett. 69, 3610 (1992).

36. "Measurement of Tau Decays Involving Eta Mesons", with M. Artuso et al.Phys. Rev. Lett. 69, 3278 (1992).

37. "Isospin Mass Splittings from Precision Measurements of $D^{*}-D$ Mass Differences", with D. Bortoletto et al.Phys. Rev. Lett. 69, 2046 (1992). 
38. "Measurement of the $D^{*}(2010)$ Branching Fractions", with F. Butler et al.Phys. Rev. Lett. 69, 2041 (1992).

39. "A Measurement of the tau lepton lifetime", with M. Battle et al.Phys. Lett. B291, 488 (1992).

40. "The CLEO II detector", with Y. Kubota et al.Nuclear Instruments and Methods A320, 66 (1992).

41. "Observation of the Decay $\Xi_{c}^{0} \rightarrow \Omega^{-} K^{+}$, with S. Henderson et al.Phys. Lett. B283, 16 (1992).

42. "Electronic Branching Ratio of the $\tau$ lepton", with R. Ammar et al.Phys. Rev. D45, 3976 (1992).

43. "Two-body $D_{s}^{+}$decays to $\eta \pi^{+}, \eta^{\prime} \pi^{+}, \eta \rho^{+}, \eta^{\prime} \rho^{+}$, and $\phi \rho^{+}$", with M. Daoudi et al.Phys. Rev. D45, 3965 (1992).

44. "Measurements of Semileptonic Branching Fractions of B Mesons at the $\Upsilon(4 \mathrm{~S})$ Resonance.", with S. Henderson et al.Phys. Rev. D45, 2212 (1992).

45. " $D_{s}^{+}$decays to $\eta \rho^{+}, \eta^{\prime} \rho^{+}$, and $\phi \rho^{+}$", with P. Avery et al.Phys. Rev. Lett. 68, 1279 (1992).

46. " $D_{s}^{+}$decays to $\eta \pi^{+}$and $\eta^{\prime} \pi^{+}$", with P. Avery et al.Phys. Rev. Lett. 68, 1275 (1992).

47. "Measurement of baryon production in $B$-meson decay", with G. Crawford et al.Phys. Rev. D45, 752 (1992).

48. "Inclusive and exclusive decays of $B$ mesons to final states including charm and charmonium mesons", with D. Bortoletto et al.Phys. Rev. D45, 21 (1992).

49. "Shape Studies of Quark Jets vs. Gluon Jets at $\sqrt{s}=10 \mathrm{GeV}$ ", with M.S. Alam et al.Phys. Rev. D46, 4822 (1992).

50. "Exclusive $\chi_{b}(2 P)$ Production in $\Upsilon(3 S)$ decays", with G. Crawford et al.Phys. Lett. B294, 139 (1992).

51. "A muon identification detector for $B$-physics near $e^{+} e^{-} \rightarrow B \bar{B}$ threshold", with $\mathrm{D}$. Bortolleto et al.Nucl. Instr. \& Methods, A320, 114 (1992).

52. "Unusual decay modes of $D^{0}$ and $D^{+}$mesons.", with R. Ammar et al.Phys. Rev. D44, 3383 (1991).

53. "Measurement of the ratio $\mathrm{B}\left(D^{0} \longrightarrow K^{*-} e^{+} \nu\right) / \mathrm{B}\left(D^{0} \longrightarrow K^{-} e^{+} \nu\right)$ ", with G. Crawford et al.Phys. Rev. D44, 3394 (1991).

54. "Inclusive $\chi(2 P)$ Production in $\Upsilon(3 S)$ Decay.", with R. Morrison et al.Phys. Rev. Lett. 67, 1696 (1991).

55. "Measurement of the Inclusive $B^{*}$ Cross Section above the $\Upsilon(4 S)$. , with D.S. Akerib et al.Phys. Rev. Lett. 67, 1692 (1991).

56. "Study of Continuum $\mathrm{D}^{*+}$ Spin Allignment.", with Y. Kubota et al.Phys. Rev. D44, 593 (1991).

57. "Inclusive Production of the Charmed Baryon $\Lambda_{\mathrm{c}}^{+}$from $e^{+} e^{-}$Annihilations at $\sqrt{s}=10.55$ GeV.", with P. Avery et al.Phys. Rev. D43, 3599 (1991).

58. "Study of $\pi^{+} \pi^{-}$transitions from the $\Upsilon(3 S)$ and a search for the $h_{b}$ ", with I.C. Brock et al.Phys. Rev. D43, 1448 (1991).

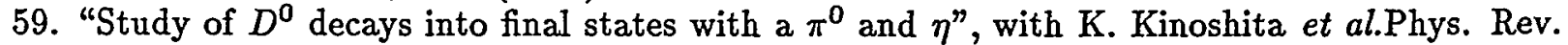
D43, 2836 (1991).

60. "Exclusive and Inclusive Semileptonic Decays of B Mesons to D Mesons.", with R. Fulton et al.Phys. Rev. D43, 651 (1991).

61. "Study of the Decays $\mathrm{D}^{0} \rightarrow \mathrm{K} \overline{\mathrm{K}}, \pi \bar{\pi}$ ", with J. Alexander et al.Phys. Rev. Lett. 65, 1184 (1990).

62. "Determination of $\mathrm{B}\left(\mathrm{D}_{s}^{+} \rightarrow \phi \pi^{+}\right)$via Observation of $\mathrm{D}_{s}^{+} \rightarrow \phi \ell^{+} \nu$ ", with J. Alexander et al.Phys. Rev. Lett. 65, 1531 (1990).

63. "Measurement of the $\Lambda_{c}^{+}$Decay-Asymmetry Parameter", with P. Avery et al.Phys. Rev. Lett. 65, 2842 (1990). 
64. "Study of $\Pi^{*}$ Production in $\tau$ Decays", with M. Goldberg et al.Phys. Lett. B251, 223 (1990).

65. "Observation of $\Upsilon(4 S)$ Decays into Non $-B \vec{B}$ Final States Containing $\psi$ Mesons", with J. Alexander et al.Phys. Rev. Lett. 64, 2226 (1990).

66. "Exclusive and Inclusive Decays of $B$ Mesons into $D_{s}$ Mesons", with D. Bortoletto et al.Phys. Rev. Lett. 64, 2117 (1990).

67. "Measurement of $\gamma \gamma$ Widths of Charmonium States", with W.Y. Chen et al.Phys. Lett. 243B, 169 (1990).

68. "Radiative $\Upsilon(1 S)$ Decays", with R. Fulton et al.Phys. Rev. D41, 1401 (1990).

69. "Search for Neurinoless Decays of the $\tau$ Lepton", with T. Bowcock et al.Phys. Rev. D41, 805 (1990).

70. "P-wave Charmed Mesons in $e^{+} e^{-}$Annihilation", with P. Avery et al.Phys. Rev. D41, 774 (1990).

71. "Observation of B-Meson Semileptonic Decays to Noncharmed Final States", with R. Fulton et al.Phys. Rev. Lett., 64, 16 (1990).

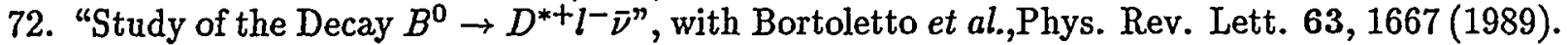

73. "Search for $b \rightarrow u$ Transitions in Exclusive Hadronic $B$-Meson Decays", with D. Bortoletto et al.,Phys. Rev. Lett. 62, 2436 (1989).

74. " $B^{\circ} \bar{B}^{\circ}$ Mixing at the $\Upsilon(4 S)$ ", with M. Artuso et al.,Phys. Rev. Lett. 62, 2233 (1989).

75. " $\Sigma_{c}^{++}$and $\Sigma_{c}^{0}$ Production from $e^{+} e^{-}$Annihilation in the $\Upsilon$ Energy Region", with $T$. Bowcock et al., Phys. Rev. Lett. 62, 1240 (1989).

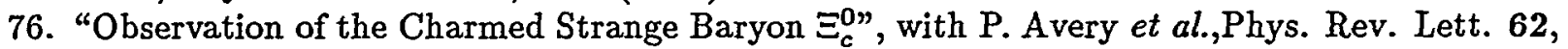
863 (1989).

77. "Search for the Charmless Decays $B \rightarrow p \bar{p} \pi$ and $p \bar{p} \pi \pi$ ", with C. Bebek et al., Phys. Rev. Lett., 62, 8 (1989).

78. "Search for a Neutral Higgs Boson in B Meson Decay", with M.S. Alam et al.,Phys. Rev. D40, 712 (1989), erratum ibid D40, 3790 (1989).

79. "Search for the Production of Fractionally Charged Particles in $e^{+} e^{-}$Annihilations at $\sqrt{s}=$ $10.5 \mathrm{GeV}$ ", with T. Bowcock et al.,Phys. Rev. D40, 263 (1989).

80. "Measurement of the Muonic Branching Fractions of the $\Upsilon(1 S)$ and $\Upsilon(3 S)$ ", with W.-Y. Chen et al., Phys. Rev. D39, 3528 (1989).

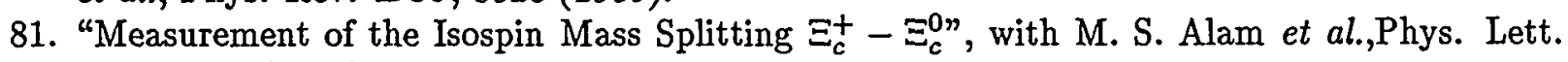
226B, 401 (1989).

82. "Measurement of $D_{s}$ Decay Modes", with W.-Y. Chen et al.,Phys. Lett. 226B, 192 (1989).

83. "First Observation of Inclusive $\psi$ Production in $\Upsilon$ Decays", with R. Fulton et al.,Phys. Lett. 224B, 445 (1989).

84. "A Search for Exclusive Penguin Decays of B Mesons", with P. Avery et al.,Phys. Lett. 223B, 470 (1989).

\section{CRYSTAL BALL EXPERIMENT}

85. "Measurement of the branching ratios for the decays $\tau \rightarrow$ hadron $\pi^{\circ} \nu$ and $\tau \rightarrow$ hadron $\pi^{\circ} \pi^{\circ} \nu^{\prime \prime}$, with A. Antreasyan et al.SLAC-PUB-5403, DESY 91-001, Dec. 1990, accepted by Phys. Lett. B.

86. "Measurement of $\pi^{\circ}$ and $\eta$ meson production in $e^{+} e^{-}$annihilation at $\sqrt{s}$ near $10 \mathrm{GeV}$ ", with Ch. Bieler et al.Z. Phys. C49, 225 (1991).

87. "Measurement of the direct photon spectrum from Upsilon (1S) decays.", with A. Bizzeti, et al.Phys. Lett. B267 286 (1991).

88. "First observation of the reaction $\gamma \gamma \rightarrow \pi_{2} \rightarrow \pi^{\circ} \pi^{\circ} \pi^{\circ "}$, with A. Antreasyan et al.Z. Phys. C48, 561 (1990). 
89. "Observation of the exclusive decay $B \rightarrow e \nu D^{*}$ and search for $B \rightarrow e \nu \pi^{\circ}$, with A. Antreasyan et al.Z. Phys. C48, 553 (1990).

90. "Limits on Axion and Light Higgs Boson Production in $\Upsilon(1 S)$ Decays", with D. Antreasyan et al.Phys. Lett. B251, 204 (1990).

91. "Observation of a New $\eta \pi^{\circ} \pi^{\circ}$ Resonance at $1900-\mathrm{MeV} / \mathrm{c}^{2}$ in Two Photon Scattering", with K. Karch et al.Phys. Lett. B249, 353 (1990).

92. "Inclusive $J / \psi$ Production in Decays of $B$ Mesons" with W. Maschmann et al.Z. Phys. C46, 555 (1990).

93. "A Measurement of $\pi^{\circ} \pi^{\circ}$ Production in Two Photon Collisions", with H. Marsiske et al.Phys. Rev. D41, 3324 (1990).

94. "The Michel Parameter for the Decay $\tau \rightarrow e \nu \bar{\nu}$ ", with H. Janssen et al., Phys. Lett. 228B, 273 (1989).

95. "The Electron Spectrum From B Meson Decays", with K. Wachs et al., Z. Phys. C42, 33 (1989).

\section{OTHER PAPERS}

96. "Upsilon Spectroscopy: Transitions in the Bottomonium System", with D. Besson,Annual Review of Nuclear and Particle Science 43, 333 (1993).

97. "Readout Techniques and Radiation Damage of Undoped CsI", with C.L. Woody et al.,IEEE Transactions on Nuclear Science, 37, 492 (1990). 


\section{Curriculum Vitae}

Thomas Edward Coan

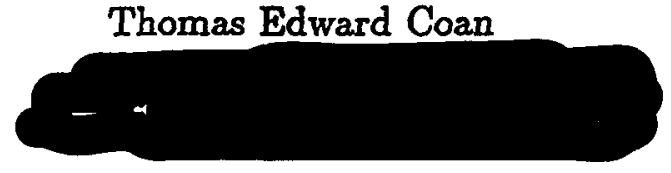

Education

S.B. in Physics, Massachusetts Institute of Technology, 1980

M.S., University of California at Berkeley, 1983

Ph.D., University of California at Berkeley, 1989

\section{Research, Teaching and Professional Experience}

- Southern Methodist University, Dallas, Texas

1994 - present: Assistant professor of Physics.

Member of the CLEO collaboration at CESR.

- Los Alamos National Laboratory

1991 -1994: Physics Postdoctoral Fellow, P-2 Group and Un-paid Associate at CERN.

Member of the L3 collaboration at LEP.

I was one of the 4 principal physicists responsible for the 1993 installation and on-line commissioning of the L3 silicon micro-vertex detector.

I initiated and conducted a search for a class of rare $\mathrm{Z}$ decays with hard photon emission sensitive to possible violations of the PCAC theorem.

- Boston University, Boston, Massachusetts

1989 - 1991: Engineering Physicist, Physics Department.

I was one of the principal mechanical designers of the drift chamber portion of the Čerenkov Ring Imaging Detector for the end-cap portion of the SLD detector at SLC.

I performed the initial mechanical stress and strain analyses of the TEXAS collaboration's scintillating fiber calorimeter proposed for the SSC.

- University of California at Berkeley

1982-1989: Research Assistant, Physics Department.

From May 1983 to September 1988, I was responsible for the mechanical design, construction and integration of the $2,000 \mathrm{~kg}$, high altitude balloon borne EXAM detector designed to search for extragalactic, heavy antimatter $(Z \sim-26)$ as a direct test of cosmological baryon number (non)conservation. I built and tested several $1.5 \mathrm{~m}^{2}$ scintillation counters, hundreds of $2-4$ meter long drift tubes, a $3 \mathrm{~m}^{3}$ Čerenkov light integration box, and $27 \mathrm{~m}^{2}$ precision frames for CR-39 track etch detectors. 


\section{Publications in Refereed Journals for Thomas Coan}

- M. Acciarri et al., L3 Collab., "Measurement of Cross-sections and Leptonic Forward - Backward Asymmetries at the Z Pole and Determination of Electroweak Parameters," Z. Phys. C 62 (1994) 551.

- A. Adam et al., L3 Collab., "A Study of Four Fermion Processes at LEP," Phys. Lett. B 321 (1994) 283.

- O. Adriani et al., L3 Collab., " $\chi_{c}$ Production in Hadronic Z Decays," Phys. Lett. B 317 (1993) 467.

- S.P Ahlen et al., "Flight Performance of EXAM - a balloon-borne detector to search for extragalactic antimatter," Nucl. Instr. and Meth. A345 (1994) 156.

- A. Adam et al., "Test Beam Results from the Prototype L3 Silicon Microvertex Detector," Nucl. Instr. and Meth. A344 (1994) 521.

- O. Adriani et al., L3 Collab., "Measurement of the Average Lifetime of B Hadrons," Phys. Lett. B 317 (1993) 474.

- O. Adriani et al., L3 Collab., "Search for Lepton Flavor Violation in Z Decays," Phys. Lett. B 316 (1993) 427.

- O. Adriani et al., L3 Collab., "An S Matrix Analysis of the Z Resonance," Phys. Lett. B 315 (1993) 494.

- O. Adriani et al., L3 Collab., "Inclusive Search for the Charmless Radiative Decay of the b-quark (b $\rightarrow \mathrm{s} \gamma)$," Phys. Lett. B 317 (1993) 637 .

- O. Adriani et al., L3 Collab., "Determination of the Effective Electroweak Mixing Angle from Z Decays," Phys. Lett. B 309 (1993) 451.

- O. Adriani et al., L3 Collab., "Measurement of $\Gamma_{b t} / \Gamma_{\text {had }}$ from Hadronic Decays of the Z," Phys. Lett. B 307 (1993) 237.

- O. Adriani et al., L3 Collab., "Search for a $\mathbf{Z}^{\prime}$ at the $\mathbf{Z}^{0}$ Resonance," Phys. Lett. B 306 (1993) 187.

- O. Adriani et al., L3 Collab., "Search for Narrow Vector Resonances in the Z Mass Range," Phys. Lett. B 313 (1993) 326.

- O. Adriani et al., L3 Collab., "Results from the L3 experiment at LEP," submitted to Physics Reports. 
- O. Adriani et al., L3 Collab., "A Search for the Neutral Higgs Boson at LEP," Phys. Lett. B 303 (1993) 391.

- O. Adriani et al., L3 Collab., "Determination of Quark Electroweak Couplings from Direct Photon Production in Hadronic Z Decays," Phys. Lett. B 301 (1993) 136.

- O. Adriani et al., L3 Collab., "Search for Anomalous Production of Single-Photon Events in $e^{+} e^{-}$-Annihilations at the Z Resonance," Phys. Lett. B 297 (1992) 469.

- O. Adriani et al., L3 Collab., "Search for Isosinglet Neutral Heavy Leptons in Z $\mathrm{Z}^{0}$ Decays," Phys. Lett. B 295 (1992) 371.

- O. Adriani et al., L3 Collab., "Search for Non-Minimal Higgs Bosons in Z" Decays," Z. Phys. C 57 (1993) 355.

- O. Adriani et al., L3 Collab., "High Mass Photon Pairs in $\ell^{+} \ell^{-} \gamma \gamma$ events at LEP," Phys. Lett. B 295 (1992) 337.

- O. Adriani et al., L3 Collab., "Searches for Non-Minimal Higgs Bosons in Z" Decays," Phys. Lett. B 294 (1992) 457.

- O. Adriani et al., L3 Collab., "A Measurement of $\tau$ Polarization in $\mathrm{Z}^{0}$ Decays," Phys. Lett. B 294 (1992) 466.

- O. Adriani et al., L3 Collab., "Isolated Hard Photon Emission in Hadronic Z" Decays," Phys. Lett. B 292 (1992) 472.

- O. Adriani et al., L3 Collab., "Determination of the number of light neutrino species," Phys. Lett. B 292 (1992) 463.

- O. Adriani et al., L3 Collab., "Measurement of the $e^{+} e^{-} \rightarrow b \bar{b}$ and $e^{+} e^{-} \rightarrow c \bar{c}$ Forward-Backward Asymmetries at the $\mathrm{Z}^{0}$ Resonance," Phys. Lett. B 292 (1992) 454.

- O. Adriani et al., L3 Collab., "Inclusive J production in $\mathrm{Z}^{0}$ Decays," Phys. Lett. B 288 (1992) 412.

- O. Adriani et al., L3 Collab., "A Test of Quantum Electrodynamics in the Reaction $e^{+} e^{-} \rightarrow \gamma \gamma(\gamma)$," Phys. Lett. B 288 (1992) 404.

- O. Adriani et al., L3 Collab., "An Improved Measurement of $B^{0}-\bar{B}^{0}$ Mixing in $\mathrm{Z}^{0}$ Decays," Phys. Lett. B 288 (1992) 395. 
- O. Adriani et al., L3 Collab., "Measurement of Inclusive $\eta$ Production in Hadronic Decays of the $\mathrm{Z}^{0}, "$ Phys. Lett. B 286 (1992) 403

- B. Alpat et al., "The design of the L3 silicon microvertex detector," Nucl. Instr. and Meth. A315 (1992) 197.

- O. Adriani et al., L3 Collab., "Determination of $\alpha_{s}$ from Hadronic Event Shapes Measured on the Z $Z^{0}$ Resonance," Phys. Lett. B 284 (1992) 471.

- O. Adriani et al., L3 Collab., "Studies of Hadronic Event Structure and Comparisons with QCD Models at the $\mathrm{Z}^{0}$ Resonance," Z. Phys. C 55 (1992) 39.

- O. Adriani et al., L3 Collab., "Search for the Neutral Higgs Boson at LEP," Phys. Lett. B 283 (1992) 454.

- O. Adriani et al., L3 Collab., "A Direct Determination of the Number of Light Neutrino Families from $e^{+} e^{-} \rightarrow \nu \bar{\nu} \gamma$ at LEP ," Phys. Lett. B 275 (1992) 209.

- B. Zhou et al., "Performance of Small-Radius Thin-Wall Drift Tubes in an SSC Radiation Enviroment at the MIT Research Reactor," IEEE Transactions in Nuclear Science, 37 (1990) 1564.

- B. Zhou, A. Marin, T. Coan, J. Beatty and S. Ahlen, "Using Dimethylether as a Drift Gas in a High Precision Drift Tube Detector," Nucl. Instr. and Meth. A287 (1990) 439. 


\section{Other Publications}

- T.E. Coan, "Hard Photon production as a probe of Non-Standard Model Physics," The Fermilab Meeting, DPF92, vol. 2, p. 1278.

- B. Alpat et al., "The design of the L3 silicon microvertex detector," Nucl. Instr. and Meth. A315 (1992) 197.

- T. Coan et al., "The Manufacturing Engineering of a Hermetic Cast Fiber Calorimeter," Proceedings of the Symposium on Research and Development for the Superconducting Super Collider, Fort Worth, TX, USA, October 1990.

- B. Zhou et al., "Performance of Small-Radius Thin-Wall Drift Tubes in an SSC Radiation Enviroment at the MIT Research Reactor," Proc. of the Workshop on major SSC detectors, Tuscon, Az., 1990, p. 551.

- S. Ahlen et al., "Design and Performance of Drift-Tubes for Tracking at High Luminosity Hadron Colliders," Particle World, 1 (1990) 168.

- D.P. Snowden-Ifft et al., "Preliminary Results from the Automated Scan of CR-39 Plastic Aboard the EXAM (Extragalactic Antimatter Detector)," Proc. 21st Inter. Cosmic Ray Conf., Adelaide, Australia, 3 (1990) 304.

- B. Zhou et al., "Using DME as a Drift Gas in a High Precision Drift Tube Detector," Proc. 21st Inter. Cosmic Ray Conf., Adelaide, Australia, 4 (1990) 418. 


\section{Report of Research Activities and Proposal for High Energy Physics}

at Southern Methodist University

\section{Theory Task B:}

K. Hornbostel, G. McCartor, F. Olness, and R. Vega 


\section{Table of Contents \\ Theory Task B:}

Project Overview

Overview

SMU Theory Highlights 1992-1994

Executive Summary

Progress Report and Proposal:

DOE PI Personnel

Kent Hornbostel $\quad 6$

Gary McCartor $\quad 9$

Fredrick Olness $\quad 16$

Roberto Vega 26

Other SMU Theoretical Personnel

Benjamin Grinstein $\quad 35$

Stephan Riemersma $\quad 36$

Doris Rosenbaum $\quad 40$

Vigdor Teplitz $\quad 40$

Ariel Zhitnitsky $\quad 42$

Budget

Budget Explanation $\quad 44$

Current and Pending Support $\quad 46$

Publications

$\begin{array}{ll}\text { Refereed Publications } & 47\end{array}$

$\begin{array}{ll}\text { Other Publications } & 50\end{array}$

Appendices

Conference \& Seminar Presentations $\quad 52$

SMU High Energy Seminar Schedule $\quad 55$

Curriculum Vitae: DOE PI Personnel $\quad 56$

Curriculum Vitae: Other SMU Theoretical Personnel 


\section{PROGRESS REPORT AND PROPOSAL: THEORY TASK}

K. Hornbostel, G. McCartor, F. Olness, and R. Vega

\section{OVERVIEW}

We summarize research results for the three year period, 1991-4, for PI's Hornbostel, $\mathrm{McCartor}$, Olness, and Vega. We propose research for the new grant period and provide a restrained budget appropriate to the work. We also review research for Grinstein, Riemersma, Rosenbaum, Teplitz, and Zhitnitsky.

The theory group at SMU has been very productive, both in terms of research and growth; during the period since May 1992, it has enjoyed support from the DOE University program. In recent years, the SMU theory group has grown to five tenured or tenuretrack theoretical faculty: Hornbostel ('93), McCartor('90), Olness('91), Teplitz('90), and Vega('92). In addition, we have Dr. Ariel Zhitnitsky as a senior research associate, Dr. Stephan Riemersma as a postdoctoral research associate, and Dr. Doris Rosenbaum as a Scholar in Residence. Our first postdoc, Dr. David Robertson, has moved to a postdoc position at Ohio State University, and Visiting Assistant Professor Gilles Couture is presently on faculty at Quebec University. Additionally, Professor Benjamin Grinstein was a member of the SMU faculty for much of 1994, and has now moved to UC-San Diego.

With this group of faculty and visitors, the theoretical research performed at SMU covers a variety of areas. Despite the setback due to the loss of the SSC, the SMU group continues to thrive. The SMU administration approved filling an experimental tenuretrack position (Tom Coan) and conversion of Professor Hornbostel from a visiting position to a theory tenure-track position after the death of the SSC. We believe that the group is sufficiently productive to warrant two theory postdocs; however, in view of what appears to be a budget crisis for the DOE University program, we request support for only one theory postdoc in the first year of the new grant period. We also request partial support for one promising theory graduate student.

McCartor and Hornbostel are active in light-cone physics. Hornbostel also works on lattice QCD in which he collaborates with Peter Lepage at Cornell. Olness and Riemersma work on QCD radiative corrections and the determination of structure functions for hadrons. Olness has played a leading role in the CTEQ national collaboration. Vega is active in electroweak symmetry breaking, CP violation, and SSC physics. Recently Vega has also been working closely with the experimental group to try to understand data for $\tau$-decay to three leptons; he has been joined in this effort by Duane Dicus (UTAustin). Zhitnitsky has a variety of interests, including: QCD Sum Rules, CP violation in the Weinberg model, instanton physics, and axion physics on which he is working with Stroynowski on an innovative detection experiment. Rosenbaum and Teplitz work on the dark matter problem, and Teplitz also collaborates with Duane Dicus (UT-Austin) on other topics. SMU now has by far the most active high-energy theory group in the northern half of the state. It constitutes a major regional resource and center of activity. 


\section{1) SMU Theory Highlights 1992-94:}

- February 1992: DOE begins support of SMU high energy experimental program.

- February 1992: SSC extends Teplitz position on international coordination; salary freed used to hire visiting Assistant Professor Hornbostel.

- May 1992: DOE awards first SMU theory grant.

- May 1992: SMU hosts Workshop On Light-Cone Quantization.

- June 1992: Lightner-Sams Foundation gives department 3-year \$50K grant.

- August 1992: SMU is the site for the 1992 XXVI International Conference on High Energy Physics (Rochester Conference).

- September 1992: Five graduate students arrive for re-instituted Ph.D. program recommended by ad hoc advisory panel of J. Toll, S. Smith, and A. Gleeson.

- March 1993: Fredrick Olness wins an SSC Fellowship for the year 1993-94. Salary savings is used for theory postdoc support.

- April 1993: TNRLC awards $\$ 120 \mathrm{~K}$ research infrastructure grant to SMU physics, including $\$ 40 \mathrm{~K}$ for support of theory postdocs.

- September 1993: Postdoc D. Robertson (SMU 1990-93) moves to postdoc position at Ohio State. Former Visiting Assistant Professor G. Couture (1990-1992) now on faculty at Quebec University.

- September 1993: Doris Rosenbaum and Vigdor Teplitz are the only Americans to win the UK Science Minister's contest for a one page explanation of Higgs Boson.

- October 1993: Department Visiting Committee including chair, M. Goldberger (UCSan Diego), R. Schwitters (then SSC, now U. of Texas), and S. Weinberg (U. of Texas), call Department's faculty "unusually talented" and progress "remarkable."

- January 1994: SMU moves Hornbostel to tenure-track position, and hires experimentalist Tom Coan.

- Jan.-Aug. 1994: Benjamin Grinstein on faculty at SMU, (DOE-supported).

- July 1994: Zhitnitsky and Stroynowski receive DOE funding for preliminary study on innovative non-accelerator axion detection experiment.

- July 1994: SMU receives a $\$ 170 \mathrm{~K}$ grant from DOE (joint with UT-Arlington) to study future use of SSC assets to aid science teaching and research in Texas.

- September 1994: Olness invited to participate in D-ZERO Collaboration QCD group workshop.

- September 1994: Department begins using non-renewable resources to honor twoyear committments to two theory postdocs.

- In the past grant period, SMU theorists have presented 53 seminars, colloquia, and conference talks at a variety of institutions.

- The theory group has published 46 papers during the past grant. 


\section{2) Executive Summary}

Members of the high energy theory group at SMU have worked on a relatively broad range of topics, having in common a fairly close connection to experimental physics. Interests include perturbative Quantum Chromodynamics (QCD) and the systematic analysis of hadronic scattering; nonperturbative QCD, including lattice calculations of heavy quark systems, and light-cone quantization applied to bound states; and studies of electroweak symmetry breaking (EWSB).

Olness has been participating in a collaboration ${ }^{*}$ which includes both experimentalists and theorists, whose long term program is the systematic analysis of data from high energy hadronic experiments in the theoretical context of QCD. Their goal is to extract the maximal amount of information about the theory from currently available experimental data, as well as to prepare for and to guide future experiments. This work includes detailed measurement of hadronic structure functions, consistent inclusion of radiative corrections to next-to-leading order, and elimination of ambiguities in choice of renormalization scheme and scale. Such a systematic program is essential for resolving current outstanding discrepancies between theoretical predictions and data, or identifying new physical phenomena where such resolution is not possible. Specifically, Olness and collaborators propose: to extend QCD calculations of deep inelastic scattering (DIS) beyond leading-order, and to compare these calculations with results from hadronic fixed-target and collider experiments; to analyze energy correlations in semi-inclusive DIS processes at the HERA electron-proton collider and transverse momentum distributions using Sudakov form factors; and to identify and search for signatures of new phenomena predicted by extensions of the Standard Model such as Supersymmetry, Left-Right models, Grand Unịied Models, and nonminimal Higgs models.

Despite the remarkable successes of the Standard Model, the mechanism by which electroweak symmetry is broken remains a mystery. The only certainty is that evidence for the mechanism of EWSB must manifest itself below the $1 \mathrm{TeV}$ energy scale. Just how that evidence may manifest itself has been the dominant subject of Vega's research efforts. Vega and collaborators were the first to obtain accurate predictions for the yield of $W$ gauge boson pairs at future high energy colliders such as the LHC; they developed new calculational techniques essential for the study of processes involving the scattering of gauge bosons and the production of top-quark pairs; and they have carried out systematic studies to determine what it will take to discover Higgs particles over the entire mass range $50<m_{H}<1000 \mathrm{GeV}$. More recently, Vega and collaborators established that, at the LHC, discovery of at least one of the Higgs bosons of the minimal SUSY model is guaranteed over the entire range of parameter space. Prior to their work this was the subject of much debate. Vega has worked on a variety of other subjects, including CP violation in the Higgs sector, the chiral quark model, the connection between $\mathrm{CP}$ and the custodial SU(2) symmetry, and the physics of the Tau lepton. His future plans call for continuing his work on electroweak symmetry breaking, with and without $\mathrm{CP}$ violation, and the use of effective Lagrangians for parametrizing physics beyond the standard model.

Hornbostel's current and planned research pursues nonperturbative QCD along two lines. The first employs the well-established tool of lattice gauge theory on heavy quark bound states, but using a nonrelativistic effective Lagrangian to simulate the quarks, as

* CTEQ: Collaborative Theoretical and Experimental Studies of QCD. 
well as several recent innovations that allow accurate simulations on relatively modest lattices. Thus far, Hornbostel and collaborators have determined: $\alpha_{s}$ at the $Z$ mass to within $2 \%$; the $b$-quark mass to within a few per cent; $\Upsilon 1 P-1 S$ and $2 S-1 S$ splittings to the same accuracy; spin splittings and second excited states to $20-30 \%$; predictions for the as yet unobserved ${ }^{1} S_{0}$ and ${ }^{1} D_{2}$ states; and $\Upsilon$ wavefunctions. New efforts will include calculation of the $\psi$ spectrum, the use of wavefunctions extracted from simulations to compute leptonic decay rates and electromagnetic transitions, and a study of heavy-light systems such as $D$ and $B$ mesons, including calculation of form factors (Isgur-Wise functions). The second line of research exploits the boost invariance and simple vacuum of light-cone quantization (LCQ) in nonperturbative calculations. This has included the numerical computation of the masses and wavefunctions for essentially complete spectra of mesons and baryons for $1+1$ dimensional QCD, and development of a procedure to incorporate vacuum effects in the LC Hamiltonian, applied successfully to the Gross-Neveu and Schwinger models. Future work will include the study of heavy flavor components in light hadrons in $1+1$ dimensions, and strong coupling calculations of hadronic properties using a Hamiltonian LC lattice, based on previous work deriving a Hamiltonian in a light-cone temporal gauge.

McCartor has been active in the field of light-cone quantization. His work, and the work of others, over the past few years has greatly clarified the ways in which the existence of vacuum structure, condensates and spontaneous symmetry breaking appears in the light-cone representation. The role of zero modes in formulating a proper light-cone initial value problem has also been clarified. Most of the issues involving vacuum structure and zero modes - issues which McCartor and collaborators were among the first to raise - have now been resolved for field theories in $1+1$ dimensions. Work to apply the results of these studies to higher dimensions has begun, and promises to produce useful results for QCD.

As will be evident throughout this proposal, the productivity of the group has been due in large part to collaboration with our postdoctoral researchers, short-term appointments, and increasingly to members of our newly reinstituted graduate program. In order to continue their contributions, we are requesting funding for one postdoctoral position for the first 1.5 years, increasing to two for the remainder, and one graduate student. All of the members of our group are participants in worldwide collaborations. We are requesting sufficient travel money and some funds for visitors to remain active in these collaborations. As is now common, we are dependent on computer resources for a wide variety of functions, from numerical work to communication to publication. We are asking for modest funds for equipment and maintenance of these resources, and for $25 \%$ support for our system manager, whom we share with the experimental group. To this point, a large fraction of these expenses have been covered by university start-up funds. As we are now becoming an established group, this support is ending and we must rely on external funds to continue them. Finally, we are requesting two months of salary for each member to allow us to continue our research during the summer. 


\section{1) Kent Hornbostel:}

\section{Progress Report and Proposal}

QCD has provided a successful description of the strong interactions at short distances, where perturbation theory is applicable. Calculations have proven more challenging in cases where the coupling is strong, preventing comparisons of such basic quantities as hadronic masses with experiment. My current and planned research pursues nonperturbative QCD along two lines, summarized below. The first employs lattice gauge theory in a study of heavy quark systems, the second uses light-cone quantization.

\subsection{Precision Calculations of $\Upsilon$ Properties, $M_{b}$, and $\alpha_{s}$ (Hornbostel)}

\section{InTRODUCTION AND PAST RESEARCH:}

This work employs the well-established tool of lattice gauge theory on heavy quark bound states, but using a nonrelativistic effective Lagrangian to simulate the quarks. The lattice provides a nonperturbative, gauge-invariant regulator, while the effective Lagrangian exploits the essentially nonrelativistic nature of these systems. Excitations on scales greater than the heavy-quark mass, which is much greater than typical bound-state momenta, are eliminated and replaced by a finite series of local and calculable effective interactions. My collaborators and I have computed these to account for relativity and lattice-spacing errors [1] such that computations to within errors of $10 \%$, and in many cases less than $5 \%$, were possible, allowing meaningful comparisons with the wealth of experimental data available for the $\psi$ (charmonium) and $\Upsilon$ (bottomonium) systems.

After concluding a year of code development, Monte Carlo simulations at the Ohio supercomputer facility, and statistical analysis of the resulting propagators, essentially all of the original objectives of this program have been obtained for the $\Upsilon$ system. Many were presented at Lattice 93 [2] and appear in Ref. [3]. These include $\Upsilon 1 P-1 S$ and $2 S-1 S$ splittings to within a few per cent; spin splittings and second excited states to $20-30 \%$; predictions for the as yet unobserved ${ }^{1} S_{0}$ and ${ }^{1} D_{2}$ states; and $\Upsilon$ wavefunctions, which will provide input for decay rates as well as a first-principles test of various potential models.

One of the most important consequences of this study is the ability to extract several of the fundamental parameters of the standard model. We have recently submitted for publication values for the $b$-quark mass $M_{b}$, accurate to within a few per cent [4], and for the strong coupling constant $\alpha_{s}$, which we have determined to within $2 \%$ at the scale of the $Z$ mass [5]. These are currently the most accurate values available from lattice calculations or from any other method.

This project employed several new or relatively untested improvements in lattice calculational techniques, and its success suggests their utility in a broad variety of contexts. In addition to an effective nonrelativistic action, we make important use of lattice perturbation theory, improved actions to account for finite spacing errors and relativity, and smeared propagator sources based on potential model wavefunctions. In particular, the accuracy of our results depended crucially on recent advances in the choice of both perturbative scheme and scale, and the improvement of lattice operators to remove tadpoles and so bring them closer to their continuum equivalents [6]. The success of these recent advances greatly extends the range and accuracy of calculations possible on currently available lattices.

PROPOSED RESEARCH AND ONGOING WORK: 
In the near future, we intend to use improved statistics from additional runs and the implementation of the next order relativistic and lattice-spacing corrections to the Lagrangian to reduce errors in spin splittings, $M_{b}$ and $\alpha_{s}$ by factors of two to five. New efforts will include calculation of the $\psi$ spectrum; the use of wavefunctions extracted from our simulations to compute leptonic decay rates and electromagnetic transitions; and a study of heavy-light systems such as $D$ and $B$ mesons. This last will provide a firstprinciples calculation of the form factors for these mesons (Isgur-Wise functions) now determined phenomenologically.

\subsection{Light-Cone Quantized QCD (Hornbostel)}

\section{INTRODUCTION AND PAST RESEARCH:}

This line of research explores the application of light-cone quantization (LCQ) to bound states and studies of vacuum properties. Introduced in the 1960s, LCQ has long proven a useful tool in perturbation theory. However, its boost invariance and the simplicity of the LC vacuum are substantial advantages for nonperturbative calculations, and have recently encouraged some new applications. Toward that end, S. Brodsky, H.-C. Pauli and I have applied this formalism to numerically compute masses and wavefunctions in $1+1$ dimensional QCD [7]. We produced the masses and wavefunctions for essentially complete spectra of mesons and baryons for a variety of numbers of colors, typically at the cost of a few minutes of CPU time. I have also developed a procedure for identifying and retaining vacuum effects in the LC Hamiltonian [8], and applied it successfully to the Gross-Neveu and Schwinger Models [9,10]. Finally, the Hamiltonian lattice formulation of QCD at strong coupling provides a simple and appealing picture of' confinement [11]. To see how this picture appears on the light cone, I have completed the necessary first step of working out quantization in the LC equivalent of the temporal gauge [12].

\section{PROPOSED RESEARCH AND ONGOING WORK:}

I have expanded the QCD $(1+1)$ code to include an arbitrary number of flavors as well as colors. Among other projects, Brodsky and I propose to use it to study higher-Fock wavefunctions, particularly those with additional heavy quarks. In addition, I would like to generalize the technique used to retain vacuum effects in the Gross-Neveu and Schwinger Models in the development of an effective Hamiltonian which allows for a simple vacuum but incorporates these effects into additional interactions. Applied to QCD, this would retain properties due to vacuum dynamics, such as chiral symmetry breaking, while preserving some of the simplicity of LCQ. Robertson, McCartor and I continue to develop a method for consistently including boundary degrees of freedom in LCQ; in contrast to equal-time quantization, these degrees of freedom often play an important physical role. Finally, I intend to use the temporal-gauge version of LCQ to set up a three-dimensional LC lattice, which will provide a gauge-invariant regulator. I will employ this in a strongcoupling calculation of hadrons, which will contain linear confinement already in lowest order. 


\subsection{References:}

[1] Improved Nonrelativistic QCD for Heavy Quark Physics, G. P. Lepage, L. Magnea, C. Nakhleh, U. Magnea and K. Hornbostel, Phys. Rev. D 46, 4052 (1992).

[2] Precision $\Upsilon$ Spectroscopy and Fundamental Parameters from NRQCD, G. P. Lepage and J. Sloan, poster sessions at Lattice '93; NRQCD Collaboration, Nucl. Phys. B (Proc. Suppl.) 34 417, (1994).

[3] Precision $\Upsilon$ Spectroscopy from Nonrelativistic Lattice $Q C D$, C. T. H. Davies, K. Hornbostel, A. Langnau, G. P. Lepage, A. Lidsey, J. Shigemitsu, and J. Sloan, SCRI-94-39, OHSTPY-HEP-T-94-005, hep-lat/9406017, to appear in Phys. Rev. D (1994).

[4] New Determination of $M_{b}$ From Lattice $Q C D$, C. T. H. Davies, K. Hornbostel, A. Langnau, G. P. Lepage, A. Lidsey, C.J. Morningstar, J. Shigemitsu, and J. Sloan, SCRI-94-57, OHSTPY-HEP-T-94-004, heplat/9404012, to appear in Phys. Rev. Lett. (1994).

[5] A Precise Determination of $\alpha_{s}$ From Lattice QCD, C. T. H. Davies, K. Hornbostel, G. P. Lepage, A. Lidsey, J. Shigemitsu, and J. Sloan, OHSTPY-HEP-T-94-013, FSU-SCRI-94-79, hep-ph/9408328, submitted to Phys. Lett. (1994).

[6] G. P. Lepage and P. B. Mackenzie, Phys. Rev. D 48, 2250 (1993).

[7] Light Cone Quantized QCD in (1+1) Dimensions, K. Hornbostel, S. J. Brodsky and H.-C. Pauli, Phys. Rev. D 41, 3814, (1990).

[8] Nontrivial Vacua From Equal Time to the Light Cone, K. Hornbostel, Phys. Rev. D 45, 3781 (1992).

[9] D. J. Gross and A. Neveu, Phys. Rev. D 10, 3235 (1974).

[10] J. Schwinger, Phys. Rev. 128, 2425 (1962).

[11] J. Kogut and L. Susskind, Phys. Rev. D 11, 395 (1975).

[12] Light Cone QCD in a Temporal Gauge, K. Hornbostel, Proceedings of the SMU Workshop on Light Cone Quantization, (unpublished) Southern Methodist University, Dallas, Texas (1992). 


\section{2) Gary McCartor:}

\section{Progress Report and Proposal}

\subsection{Introduction: Light-Cone Quantization}

For several years we have been working in the field of light-cone quantization [113]. The field itself-in its current form-has grown from a few workers in the midnineteen-eighties, to something over a hundred physicists with a worldwide distribution. Enthusiasm is high with frequent meetings and very active publication.

The greatest progress over the past few years has perhaps been in the related areas of vacuum structure and proper formulation of the light-cone initial value problem. Zero modes-operators which are independent of some space-time variable, usually $x^{-}$-play an essential role in both these areas; it is in these areas that our own work has been focused. Many of the current studies stem from a paper we wrote in 1988 [1], although the issues were known to many theorists as early as the late sixties and early seventies.

As a result of detailed studies of models in low dimension, especially the Schwinger model $[14,4,15,16,11]$ and $\phi^{4}[17,18,19,8,20,21,22]$, but also $Q C D_{1+1}[2,23,3,13]$, Yukawa [6,24] and other models [7], a rather complete understanding has been gained as to how vacuum structure is accommodated in the light-cone representation. These results suggest a new method for analyzing vacuum structure in more complicated theories such as QCD; that new method will form one main element of the work proposed below. Meanwhile, careful studies of the formulation of the light-cone initial value problem have shown that some earlier formulations were incomplete or incorrect. The current studies have also provided details of some of the consequences of these omissions. Work in this area is still proceeding.

In the sections below we shall briefly describe the results so far on vacuum structure and the proper inclusion of zero modes in the light-cone initial value problem and shall describe the research we propose to do to make use of the information gained. This research will focus substantially on theories more complex than those so far studied.

\subsection{Vacuum Structure (McCartor)}

One often reads the statement-accompanied by a "proof" or not-that in the lightcone representation the physical vacuum is the perturbative vacuum. That idea forms one of the main reasons for interest in the light-cone methods, but it originally also formed a major objection to these methods: since we know of theories which have degenerate vacua, condensates, spontaneous symmetry breaking and related phenomena, a method which cannot accommodate these subtleties must be deficient. The complaint is particularly relevant in view of the fact that many of the theories thought to describe nature are presumed to possess such effects. If a "proof" of the simplicity of the light-cone vacuum is given, it comes-stated in one way or another-to this: The operator $P^{+}$has the same form as a functional of the fields that it does in the free theory. That operator is positive semidefinite and, for fields initialized on $x^{+}=0$ has only one state with lowest eigenvalue of $P^{+}$- the perturbative vacuum-so that must be the physical vacuum.

There are two arguments that $P^{+}$is the same as in the free theory: The "algebraic" argument simply notes that the density, $T^{++}$, one integrates over the surface $x^{+}=0$ to calculate $P^{+}$does not involve the interaction term in the Lagrangian (due to the fact that $g^{++}=0$ ) so if one simply calculates the answer one must get the same answer as in free 
theory. A somewhat deeper argument is that $P^{+}$is the generator of translations in the initial value surface $x^{+}=0$. Since we initialize our fields to be isomorphic to free fields on the initial value surface we know the free $P^{+}$will correctly perform these translations. So if we have a well-posed initial value problem, and thus a complete set of operators, the only possible modification of $P^{+}$would be to add a multiple of the identity which would have no effect on the dynamics.

The flaw in all these arguments is that when massless fields are present a well posed initial value problem cannot be formulated by providing initial conditions on a single characteristic such as $x^{+}=0$. Operators associated with variables which must be initialized on other surfaces commute with those initialized on $x^{+}=0$ and can therefore mix with $P^{+}$without contradicting the Heisenberg equations; states created by these operators can mix with the vacuum. The failure of the "algebraic" argument presented above is, for the case of gauge theories, somewhat subtle: while the interaction term in the Lagrangian does not enter $T^{++}$, the Fermi products which do enter, such as $\psi_{+} \partial_{-} \psi_{+}$, must be gauge corrected and in that way the interaction does enter $P^{+}$. In two dimensions the gauge correction can be made by using

$$
\lim _{\epsilon^{-} \rightarrow 0}\left(e^{-i e \int_{x}^{x+\epsilon^{-}} A_{-}^{(-)} d x^{-}} \psi_{+}^{*}\left(x+\epsilon^{-}\right) \psi_{+}(x) e^{-i e \int_{x}^{x+\epsilon^{-}} A_{-}^{(+)} d x^{-}}-\text {V.E.V. }\right) ;
$$

a current problem is to extend this procedure to higher dimensions. The failure of the "algebraic" argument is precisely like an anomaly: in defining the singular operator product one can maintain gauge invariance or the kinematical nature of $P^{+}$but not both. The only currently known method for finding the correct $P^{+}$in the interacting case involves the following steps: carefully choose a complete set of variables and initialize them as isomorphic to free fields on an appropriate set of characteristic surfaces (in the case of the continuum a modification of this procedure is necessary; this point will be discussed further in the next section); determine any constrained zero modes ( $c f$. next section) by analyzing the equations of motion; work out the necessary densities paying close attention to gauge invariance where appropriate; integrate the densities over all surfaces which contribute to $P^{+}$. The eigenstate of $P^{+}$with the lowest eigenvalue is the physical vacuum if it is nondegenerate or, if it is degenerate, some combination of these states is the physical vacuum. It may thus be possible to find the physical vacuum without solving the full dynamics. This procedure works for the Schwinger model and for $Q C D_{1+1}$.

To be more definite let us briefly outline the case of the Schwinger model [11]: we initialize $\psi_{+}$on $x^{+}=0$ and $\psi_{-}$on $x^{-}=0$ in each case as isomorphic to free fields with antiperiodic boundary conditions; we work in the gauge where $A^{+}$is independent of $x^{-}$ and find the zero modes in the gauge fields by use of the equations of motion-at this step it is crucial to use a gauge invariant current; we then solve for the interacting $P^{+}$. We find that the free $P^{+}$:

$$
P_{F R E E}^{+}=\frac{1}{4 L m^{2}} Q_{+}^{2}+2 \sum_{n=1}^{\infty} p_{-}(n) C^{*}(n) C(n)
$$

has been changed to

$$
P^{+}=\frac{1}{4 L m^{2}}\left(Q_{+}^{2}-Q_{-}^{2}\right)+2 \sum_{n=1}^{\infty} p_{-}(n) C^{*}(n) C(n)
$$

As anticipated we find that operators, $Q_{-}$, associated with $\psi_{-}-$the field which cannot be specified on $x^{+}=0$-have mixed with $P^{+}$under the interaction. The $\theta$-states 
are formed from the (degenerate) eigenstates of $P^{+}$with lowest eigenvalue. It is important to note that although the argument that the physical vacuum is the perturbative vacuum fails, all the $\theta$-states are much simpler in the light-cone representation than in the equaltime representation-simpler to find and simpler to express.

While the process of forming the interacting $P^{+}$is complicated we know from the outset that states which can mix with the vacuum must come from operators associated with the $\psi_{-}$field. It is important to note that this set of states which can mix with the vacuum grows rather slowly with the dimension of space-time: for the Schwinger model we have a line of Fermi modes; for four dimensions (and massless quarks) we have two lines of Fermi modes for each color of quark and two lines of gluons for each gluon field-the important point is that it remains a line rather than becoming a plane, a three-space, etc. We note here the important simplifications which result from the periodicity conditions: the vacuum problem involves the behavior in the infrared region; by imposing periodicity conditions one rigorously separates those modes which are infrared (zero modes) from those which are not; in the continuum where that separation is not clean the vacuum problem is technically more complex. Even in the case of the Schwinger model sophisticated methods are needed in the continuum case to treat what, in the discretized case, is a relatively straightforward problem.

We are currently working to extend these methods to higher dimension. Although easy to state, the problem is technically complex due to the necessary renormalizations. One important aspect is composite operator renormalization. I visited Padua for a month last Spring to .work -with Bassetto and collaborators who are experts in perturbative composite operator renormalization in the light-cone gauge [25]. While it is not clear that perturbative renormalization will be adequate for our needs, in the Schwinger model a perturbative renormalization of the currents is sufficient to devise a nonperturbative renormalization of $P^{+}$. That may prove to be the case in higher dimensions. The Padua group is interested in the program described above and we are collaborating. If the problem can be solved in the way we hope, the first need is a renormalized current for use in the equations of motion such as has been given for QED [26,27,28,29].

Apart from the ambitious program to determine the vacuum theoretically, the reduction in possible states with which the perturbative vacuum can mix may be useful for phenomenology or for use in association with procedures such have been suggested by Ken Wilson and colleagues [30] at Ohio State. One aspect of their procedures is to expand the hadron wave function in a set of states whose coefficients are then determined; it may be that a parameterized vacuum formed from the states discussed above would fit into that method rather naturally. I will visit Ohio State in Fall 94 to discuss that possibility and other aspects of the light-cone methods.

So far our discussion of vacuum structure has concentrated on the case where some state other than the light-cone perturbative vacuum becomes the physical vacuum. Phenomena often associated with vacuum structure in the equal-time representation can appear in a different way in the light-cone representation. The prototype case is $\phi^{4}$ with a wrong-sign mass. In the equal-time representation the vacuum shifts, at the critical point, from one state to another. In the new vacuum the field has a nonzero vacuum expectation value. In the light-cone representation the vacuum remains the perturbative vacuum through the critical point but a constrained zero mode ( $\mathrm{cf}$ next section) of the field is discontinuous so that again the field has a nonzero vacuum expectation value in the broken phase $[8,20]$. While the problem has been solved in considerable detail $[21,22]$ 
in the light-cone representation there are still some questions regarding finite volume effects. It is also worth noting that no one has even stated, much less proved the Goldstone theorem (or its equivalent) in this framework.

It is not yet clear which of the phenomena described-a vacuum state other than the perturbative vacuum or a constrained zero mode-is responsible for the VEV's in QCD. The light-cone gauge constraint equations are nonlinear and there is some potential for the phenomenon described for the case of $\phi^{4}$ to occur. On the other hand no case of that phenomenon is known where the symmetry is not broken in classical theory. It may therefore be more likely that the vacuum mixing as in the case of the Schwinger modelwhich is a purely quantum effect-may be more important. Perhaps both effects occur, but in any event the light-cone representation, where the number of states which can potentially mix with the perturbative vacuum is greatly reduced, seems a natural context in which to study the QCD vacuum and we propose to use the tools thus far developed to attempt to learn more about that subject-certainly one of the outstanding problems in the field today.

\subsection{Zero Modes}

\section{(McCartor, Robertson)}

Zero modes play a central role in questions of vacuum structure but their role in light-cone quantization is by no means limited to vacuum questions. Indeed most of the subtleties associated with light-cone quantization involve zero modes of some sort. Zero modes are of two types: true degrees of freedom which cannot be initialized on $x^{+}=0$; and terms in fields which are not in themselves new degrees of freedom-they are expressed in terms of the true degrees of freedom. The latter type of term is zero when there is no interaction but becomes nonzero due to the interaction-this type of term we call a constrained zero mode. Much has been learned about the zero modes in the last few years; the zero modes which are true degrees of freedom were first discussed in Ref. [1] while the constrained zero modes were first discussed in Ref. [17], and in the present context, in Ref. [5]. The constrained zero modes give the symmetry breaking in $\phi^{4}$. Leaving out either type of zero mode is an error, usually with severe consequences. The situation is further complicated by the fact that the continuum case is remarkably different from the case where periodicity conditions are imposed. Here we shall. briefly describe the information gained in the last several years and discuss the most important remaining issues.

One of the equations of motion in Yukawa theory is:

$$
\left(4 \partial_{+} \partial_{-}-\vec{\partial}_{\perp}^{2}+\mu^{2}\right) \phi=-g \bar{\psi} \psi
$$

If periodicity conditions are imposed, the right hand side of this equation has a zero mode. The Bose field has no zero mode as a degree of freedom; the equation is a constraint for the zero mode. If the zero mode is left out, an incorrect $P^{-}$is calculated leading to an inconsistency between the equations of motion and the Heisenberg equations, an incorrect spectrum and violations of covariance so severe that the divergent self-mass cannot be absorbed into a mass renormalization [5]. This phenomenon is very common and in particular occurs in gauge theories in the equations of motion for the gauge fields. An analyses for Maxwell's equations for QED has recently been given [31] and it is found that much the same situation holds as for Yukawa theory. One important outstanding problem is to extend these results to the nonabelian case but there are remaining issues 
for the cases so far studied-issues regarding the correct formulation of the equation and regarding solving the equation once a correct form is known.

One issue involving formulating the problem is operator ordering. When the equations are nonlinear, as in the case of QCD, questions of operator ordering arise. Usually it is assumed that these should be resolved by symmetrizing to the maximum extent possible. That is a good guess but the problem can be treated at a more fundamental level by checking consistency between the equations of motion and the Heisenberg equations. Forcing such consistency may uniquely determine the correct order or it may not. At the least it should be checked that a chosen order is one which is consistent; so far such checks have not been done. A more subtle and probably more important issue associated with formulating the equations involves renormalization of the sources, which in the case of gauge theories are currents. The studies done so far have, in dimensions greater than two, used naive definitions for the sources. It is known that in four dimensions operators associated with the gauge field such as $A^{\mu}$ and $\partial_{\nu} F^{\nu \mu}$ mix with the naive currents under renormalization $[32,26]$. The implications of that mixing to the problem of formulating the light-cone gauge constraint equations have not yet been studied. To begin with we propose to make use of the previous work on perturbative renormalization in QED to study the implications of those results to the problem of the light-cone gauge constraint equations. More generally the renormalization of the currents in nonabelian theories will have to be developed and we will have to understand whether or not perturbative renormalization of the currents is sufficient to renormalize the equations of motion we need.

In the constraint equations we have been discussing, the unknown operator (the zero mode) appears on both sides of the equation usually under an integral in one term; thus the constraint is an integral equation rather than an explicit expression for the zero mode. Solving these equations is nontrivial even for some problems in two dimensions [21] and efficient methods will have to be developed for use in realistic theories. For procedures which formulate the problem in a finite subspace as an approximation - such as TammDancoff or DLCQ - the constraints take the form of relations between finite dimensional matrices. One possibility is to use perturbation theory to solve the constraints. While it may seem a dubious procedure to use perturbation theory to formulate what is supposed to be a nonperturbative calculation, the important point is that for the finite approximations the accuracy of the solution to the constraints can be checked. If the approximate solution is more accurate than the rest of the calculation then it is sufficient. We have been talking to John Hiller who has a computer program with most of the necessary routines to do a perturbation calculation then check the accuracy of the solution for the case of DLCQ in QED. We hope to collaborate with him on this topic in Fall 94.

So far our discussion of the constrained zero modes has been for the case where periodicity conditions are imposed and thus the zero modes are isolated from the nonzero modes. For the case of the continuum, that problem formally goes away in that the constrained zero mode is now a set of measure zero and one can go all the way to the gauge where $A^{+}=0$. Furthermore, the physical degrees of freedom which cannot be specified on the surface $x^{+}=0$ are also a set of measure zero. On that basis we thought for some time that the zero mode issue went away for the case of the continuum. Within the past couple of years we have realized that thought is wrong. For the continuum, the zero modes take the form of integration constants to the constraint equations. While we have always known that such freedom was present we thought that it was consistent to set these integration constants equal to zero. It is not. Setting them to zero leads 
to inconsistency even at the level of perturbation theory. One way to state the result is that specifying the integration constants specifies the prescription for regularizing the gauge singularity - the so called spurious singularity - associated with light-cone gauge. Setting the integration constants equal to zero has the effect of specifying a prescription that Bassetto [33] and others have shown to be inconsistent. To obtain consistent results even in perturbation theory one must not initialize the gauge field as isomorphic to a free field on $x^{+}=0$ but as that plus a zero mode field [10]; to specify all components of the gauge field one needs (in four dimensions) two zero mode fields; these can be chosen to be one positive metric field and one negative metric field. In the case of QED one must not specify the Fermi field as isomorphic to a free field but as the exponential of a zero mode field times a free Fermi field [12]. For the case of two dimensions one needs only one zero mode field which turns out to be the ghost; it is interesting that this is just the ghost that is necessary to specify an operator solution to the continuum Schwinger model. We are working to extend these results to QCD and we propose to continue this work.

\subsection{References:}

[1] G. McCartor, Z. Phys. C41 (1988) 271

[2] K. Hornbostel, Ph.D. thesis, Stanford University (1988)

[3] K. Hornbostel, H.-C. Pauli, and S.J. Brodsky, Phys. Rev. D41 (1990) 3814

[4] G. McCartor, Z. Phys. C52 (1991) 611

[5] G. McCartor and D.G. Robertson, Z. Phys. C53 (1992) 679

[6] D.G. Robertson and G. McCartor, Z. Phys. C53 (1992) 661

[7] K. Hornbostel, Phys. Rev. D45 (1992) 3781

[8] D.G. Robertson, Phys. Rev.D47 (1993) 2549

[9] S.J. Brodsky, G. McCartor, H.-C. Pauli, and S. Pinsky, Particle World 3 (1993) 109

[10] G. McCartor and D.G. Robertson, Z. Phys. C62 (1994) 349

[11] G. McCartor, Schwinger Model in the Light-Cone Representation, Z. Phys. C, in press

[12] D.G. Robertson and G. McCartor, the Mandelstam-Leibbrandt Prescription in Light-Cone Quantitized Gauge Theories, preprint

[13] G. McCartor, Massless QCD1+1 in the Light-Cone Representation, Talk at the Zacopane meeting Aug. 94 - to be published in the proceedings, and Preprint in preparation

[14] T. Eller, H.-C. Pauli, and S.J. Brodsky, Phys. Rev. D35 (1987) 1493

[15] T. Heinzl, S. Krusche, and E. Werner, Phys. Lett. 256B (1991) 55

[16] F. Lenz, M. Thies, S. Levit, and K. Yazaki, Ann. Phys. 208 (1991) 1

[17] T. Maskawa and K. Yamawaki, Prog. Theor. Phys. 56 (1976) 270

[18] A. Harindranath and J.P. Vary, Phys. Rev. D36 (1987) 1141

[19] A. Harindranath and J.P. Vary, Phys. Dev. D37 (1988) 1064, 1076

[20] T. Heinzl, S. Krusche, S. Simburger, and E. Werner, Z. Phys. C56 (1992) 415

[21] C. Bender, S. Pinsky, and B. van de Sande, Phys. Rev. D48 (1993) 816 
[22] S. Pinsky and B. van de Sande, Phys. Rev. D49 (1994) 2001

[23] M. Burkardt, Nucl. Phys. A504 (1989) 762

[24] A. Harindranath, R.J. Perry, and J. Shigemitsu, OSU preprint DOE-ER-01545-56 (1991)

[25] C. Acerbi and A. Bassetto, Phys. Rev. D49 (1994) 1067

[26] W. Zimmermann, in Lectures on Elementary Particles and Quantum Field Theory, Desser, Grisaru and Pendleton, eds. (MIT, 1970)

[27] R. Brandt, Phys. Rev. 166 (1968) 1795

[28] R. Brandt, Phys. Rev. 180 (1969) 1490

[29] A. Maris, D. Dillenburg, G. Jacob, Nuovo Cimento 53A (1968) 823

[30] A recent discussion may be found in K.G. Wilson, T.S. Walhout, A. Harindranath, W. Zhang, R. Perry and S. Glazek, Phys. Rev. D49 (1994) 672 and the development may be traced through the extensive references given there.

[31] A.C. Kalloniatis and D.G. Robertson, On the Discretized Light-Cone Quantization of Electrodynamics, Heidelberg/Ohio State University preprint MPIH-V10-1994, OSU-NT- 94-03, to appear in Phys. Rev. D

[32] J.C. Collins, Renormalization (Cambridge University Press, 1984)

[33] A. Bassetto, G. Nardelli, and R. Soldati, Yang-Mills Theories in Algebraic NonCovariant Gauges (World Scientific, 1991)

[34] M. Krautgartner, H.-C. Pauli, and F. Wolz, Phys. Rev. D45 (1992) 3755 


\section{3) Fredrick Olness:}

\section{Progress Report and Proposal}

\subsection{Overview:}

Within the past decade, advances in experimental high-energy physics have provided a wealth of data on fundamental interactions. It is important that the data be completely analyzed in a coherent theoretical framework extending beyond leading-order perturbation theory. The insight gained should prove invaluable in planning experiments for the future, and, in the process, discovering unexpected "new physics."

Accordingly, the research proposed here is directed toward the interface between present high energy physics experiments and theory. The objective is to investigate both experimental complexities and theoretical subtleties in a single coherent framework to maximize the information extracted. Such a program serves: 1) to optimize the use of present and future experimental data, 2) to focus theoretical efforts toward realistic applications, and 3) to stimulate both experimentalists and theorists in discovering and resolving discrepancies between expectations and data.

This work is part of a long-term effort to incorporate advances of theoretical calculations into experimental data analysis to address two fundamental issues: 1) the structure of hadrons in the Quantum Chromodynamic-based parton model, and 2) resulting precision comparisons with new data.

\subsection{Strange Quark Content of the Proton: (Olness)}

\section{INTRODUCTION \& PAST RESEARCH:}

In references [1], [2], and [3], we computed the Next-To-Leading order (NLO) QCD contributions and found that the higher-order gluon initiated contributions can be comparable in magnitude to the "leading-order" sea-quark processes. These results have significant implications for experimental analysis. ${ }^{[4]}$

Specifically, the CTEQ-1 ${ }^{*}$ parton distribution functions ${ }^{[5]}$ (PDF's) yielded a strange quark PDF $s(x)$ comparable to the $\bar{u}$ and $\bar{d}$ PDF's, (i.e., $\kappa \simeq 1$ ). This result arose from a precise global fit including the New-Muon-Collaboration (NMC) neutral current data and the Chicago-Columbia-Fermilab-Rochester (CCFR) collaboration charged current data. Unfortunately, the available data could only determine the $s$-quark distribution indirectly. A direct determination was possible using the CCFR dimuon data. However, a NLO calculation was necessary to account for the large NLO gluon contribution, as well as to minimize the renormalization scale dependence of the problem.

A complete experimental analysis ${ }^{[6]}$ has now been completed using our formalism. While the NLO calculation does increase the $\kappa$ parameter slightly, it clearly is not consistent with $\kappa \simeq 1$. In contrast to the previous leading order analyses, they find that the strange sea $x$-dependence is similar to that of the non-strange sea. Additionally, the value of the charm mass extracted from the fit also increases, and is consistent with that determined in other processes. Certainly, this confrontation between experimental and theoretical QCD has yielded deeper theoretical understanding and more precise experimental predictions for $m_{c}, \kappa$, and the strange sea parton distribution function, $s(x)$.

* CTEQ: Collaborative Theoretical and Experimental Studies of QCD. For a more complete description of CTEQ activities, see Section 3.7. 


\section{ONGOING \& PROPOSED RESEARCH:}

Members of the CTEQ and CCFR collaborations are working together to incorporate this new data into the global PDF analyses to extract the charm mass and the strange quark distribution precisely. Some of this information ${ }^{[7]}$ is contained in the CTEQ set 2 PDF's, but a complete analysis will require a concerted effort by both CTEQ and CCFR.

\subsection{A Unified QCD Formulation of Leptoproduction of Massive Quarks: (Olness, Riemersma)}

\section{INTRODUCTION \& PAST RESEARCH:}

Applying the above work to include collider processes, we present a unified formulation of neutral and charged current phenomena that uses a physically intuitive subtraction procedure. ${ }^{[2,3]}$ While there are many subtraction prescriptions on the market, this unified formulation yields a stable, reliable prediction throughout the entire kinematic region (including the threshold region). Calculations for fixed-target and collider data indicate that most heavy quark production processes lie in this intermediate threshold region where the heavy quark neither decouples nor can be treated as "light" parton; therefore, it is extremely important to have a well-defined theoretical prediction that is insensitive to the renormalization scheme and scale. $e^{[8,9]}$

A unified QCD formulation of leptoproduction of massive quarks in charged current and neutral current processes ${ }^{[2,3]}$ involves adopting consistent factorization and renormalization schemes that encompass both vector-boson-gluon-fusion (flavor creation) and vector-boson-massive-quark-scattering (flavor excitation) production mechanisms. ${ }^{[1,11]}$ It provides a framework which is valid from the threshold for producing the massive quark (where gluon-fusion is dominant) to the very high energy regime when the typical energy scale $\mu$ is much larger than the quark mass $m_{Q}$ (where quark-scattering should be prevalent). This approach effectively resums all large logarithms of the type $\alpha_{s}(\mu) \log \left(\mu^{2} / m_{Q}^{2}\right)^{n}$ which limit the validity of existing fixed-order calculations to the region $\mu \sim \mathcal{O}\left(m_{Q}\right)$. We show that the massive quark-scattering contribution (after subtraction of overlaps) is important in most parts of the $\left\{x, Q^{2}\right\}$ plane except near the threshold region. We demonstrate that the factorization scale dependence of the structure functions calculated in this approach is substantially less than those obtained in the fixed-order calculations, as one would expect from a more consistent formulation.

\section{Ongoing \& Proposed Research:}

As an extension of the work described above, we have computed the 1-loop $\mathcal{O}\left(\alpha_{s}\right)$ corrections to the heavy quark-initiated processes for general masses and general couplings. While specialized formulas exist for charged and neutral current cases, ${ }^{[10]}$ this general result will allow us to compute both charged and neutral current processes (including Z-exchange) in a unified manner. This calculation is of particular theoretical interest because it will highlight the sensitive balance between the renormalization scheme choice for the parton distributions and the hard-scattering Wilson coefficient. At a given order, we expect the quark-initiated contributions to be less than the gluon-initiated ones because $^{[1]} f_{q}(x, \mu) \ll f_{g}(x, \mu)$. However, because the second-order Altarelli-Parisi splitting kernels $P_{q \rightarrow q}^{(2)}(x)$ and $P_{g \rightarrow q}^{(2)}(x)$ contain singular $1 / x$ terms whereas the first-order $P_{q \rightarrow q}^{(1)}(x)$ and $P_{g \rightarrow q}^{(1)}(x)$ do not, the evolution of the quark distribution at small- $x$ will be completely dominated by the second-order kernel rather than the first-order kernel. ${ }^{[12]}$ Consequently, 
contributions from higher order quark-initiated processes should prove significant in canceling the $\mu$-dependence. This work is in progress. ${ }^{[13]}$

\subsection{A Third-Order Calculation of Leptoproduction of Heavy Quarks: (Olness, Riemersma)}

\section{InTRODUCTION \& PAST RESEARCH:}

Our formalism uses the $\mathcal{O}\left(\alpha_{s}^{0}\right)$ and $\mathcal{O}\left(\alpha_{s}^{1}\right)$ heavy quark production process to obtain a NLO result. ${ }^{[2,3]}$ Riemersma and collaborators have performed a complementary calculation using the $\mathcal{O}\left(\alpha_{s}^{1}\right)$ and $\mathcal{O}\left(\alpha_{s}^{2}\right)$ contributions. ${ }^{[0]}$ We have pooled our efforts to understand the kinematic regimes of applicability of each calculation. Specifically, we compare the results of the fixed-flavor scheme (FFS) calculation ${ }^{[9]}$ with the variable-flavor scheme (VFS) calculation ${ }^{[2,3]}$ for the case of neutral-current (photon-mediated) heavy-flavor (charm and bottom) production. We examine the features of both calculations throughout phase space and compare the structure function $F_{2}\left(x, Q^{2}\right)$. We also analyze the dependence of $F_{2}\left(x, Q^{2}\right)$ on the mass factorization scale $\mu$. We find that the former (FFS) is most applicable near threshold, while the latter (VFS) works well for asymptotic $Q^{2}$. The validity of each calculation in the intermediate region depends on the $x$ and $Q^{2}$ values chosen. The results of this detailed comparison is presented in references [14] and [15].

\section{ONGOING \& PROPOSED RESEARCH:}

We note that the difference between the VFS and FFS calculations discussed above suggests higher order contributions yet to be included may be important. Combining these two calculations in a consistent fashion (with the additional mass factorizations required) will allow us to make predictions based upon a third-order result that combines the best attributes of both calculations. Such a result would be theoretically significant since there are very few processes are computed to three orders. More importantly, it would be experimentally significant as the increased luminosity at HERA allows the study of heavy quark production in detail. The combination should provide an important test of perturbative QCD when compared with the results from HERA. This calculation merges the expertise of the $\operatorname{LRSN}^{[9]}$ and ACOT ${ }^{[2,3]}$ groups, and is now in progress.

\subsection{Transverse Momentum Distributions at HERA:} (Olness)

\section{INTRODUCTION \& PAST RESEARCH:}

Semi-inclusive deeply inelastic scattering at HERA will provide a good opportunity for testing QCD. One will be able to investigate the final state for transverse momenta $q_{T}$ that are large enough that there should be little confusion from non-perturbative effects. Tests of QCD using nearly on-shell photons will also be important, but the investigation of the final state of deeply inelastic scattering should play a special role because the measurement of the structure functions such as $F_{2}\left(x, Q^{2}\right)$ in inclusive deeply inelastic scattering is so important. According to perturbative QCD, the dependence of these structure functions on $Q^{2}$ is controlled by final states containing large transverse momentum partons. These high $P_{T}$ final states occur in a fraction $\alpha_{s}$ of the events. If this theory is right, then one must be able to measure directly the energy flow in these final states.

We formulate an energy correlation observable ${ }^{[16,17,18]}$ for semi-inclusive electron-proton scattering at high momentum transfers, and compute these hadronic structure functions 
at order $\alpha_{s} .{ }^{[19]}$ This observable makes use of the large statistics that HERA will generate, is infrared-safe, and independent of any fragmentation function. Similar ideas used by Collins and Soper ${ }^{[20]}$ for inclusive $e^{+} e^{-}$annihilation into two observed hadrons, and by Davies, Webber and Stirling ${ }^{[21]}$ for the Drell-Yan process, have proved valuable. Measurement of the distribution of hadronic energy in the final state in deeply inelastic electron scattering at HERA can provide a good test of our understanding of perturbative QCD. For example, we note that the hadronic structure functions at large $q_{T}$ receive substantial contributions from incoming gluons. Thus the measurement of the energy distribution at large $q_{T}$ can provide a direct measurement of the gluon distribution in a proton. ${ }^{[22]}$ Preliminary Monte Carlo results indicate this approach can yield very sensitive tests of QCD.

\section{ONGOING \& PROPOSED RESEARCH:}

With the HERA machine now taking data, it is of interest to cover the entire range of $q_{T}$ that can be explored with the ZEUS and H1 experiments. In reference [19] we computed the $\mathcal{O}\left(\alpha_{s}\right)$ perturbative corrections to this process; however, the perturbative energy correlation structure functions included a remnant collinear singularity as $q_{T} \rightarrow 0$ so that the results were only applicable for $q_{T} / Q \gtrsim 1 / 10$. While this region of validity encompasses most of the $q_{T}$ range, the HERA events are dominantly at lower $q_{T}$ values; therefore, we are studying the case of small transverse momentum for which contributions from graphs at arbitrary order must be summed. ${ }^{[23}$

We use the approach of Collins, Soper, and Sterman ${ }^{[27]}$ which has successfully been applied to a variety of processes. This technique allows us to reorganize the perturbation expansion to sum both the leading and sub-leading logarithms such that the perturbation expansion is valid even when $\alpha_{s} \ln ^{2}\left(Q / q_{T}\right)$ is large. This calculation has been performed by Ruibin Meng (Kansas), Dave Soper (U. of Oregon), and myself. ${ }^{[28]}$ The result is an energy correlation structure function that can be measured to high precision with the HERA data, and is theoretically well defined throughout the $q_{T}$ range. A careful measurement should 1) test our ability to resum the soft gluon contributions, and 2) allow us to determine the character of the nonperturbative contributions which enter in the small $q_{T}$ region.

\subsection{Signatures of "New Physics" (Olness, Vega)}

\section{INTRODUCTION \& PAST RESEARCH:}

Without accurate comparisons between theoretical expectations and experimental results, we are incapable of distinguishing Standard Model signatures from an infinite number of extended models. There are presently a variety of extensions of the standard model which could possibly represent a logical step towards a grand unified theory. ${ }^{[29,30]}$ Such extensions of the standard model introduce new sets of gauge- and Higgs-bosons which one may hope to observe in future experiments. ${ }^{[31,32]}$ In a collection of studies, we have examined different extensions of the Standard Model at different experimental facilities ${ }^{[3-41]}$ Here I review recent efforts.

In collaboration with N. G. Deshpande (Oregon), J. Gunion (UC-Davis), and B. Kayser (NSF), we examined the predictions of the conventional left-right symmetric (LRS) model ${ }^{[12,43,44,45]}$ in the case where the gauge symmetry was broken by both bi-doublet and triplet Higgs fields. ${ }^{[4]]}$ A primary objective of this study was to identify clearly all available phenomeno- 
logical paths consistent with experimental and theoretical considerations. In the course, we uncovered new aspects of left-right models, examined some realistic manifestations of the "minimal" LR model, and imposed phenomenological constraints to establish their viability.

There has been much interest in the possibility of constructing an ultra-high-energy $e^{+} e^{-}$collider. This next generation machine would most likely consist of a pair of linear colliders with a center of mass energy of 1 to $2 \mathrm{TeV}$. In order to properly assess the potential for such a machine to discover new physics, we investigated the production of Higgs bosons of the minimal supersymmetric (SUSY) model at an $e^{+} e^{-}$collider. $^{[4]}$

Recent work has shown that the radiative corrections can significantly change the mass spectrum of the SUSY Higgs sector. ${ }^{[4,49]}$ In collaboration with A. Brignole, J. Ellis, J. Gunion, M. Guzzo, G. Ridolfi, L. Roszkowski, Fabio Zwirner, ${ }^{[50]}$ we considered the available production mechanisms for Higgs particles via an intermediate Z-boson, and also via vector-boson fusion in the context of the effective- $W$ approximation ${ }^{[51]}$ Incorporating 1-loop corrections into the analysis of Higgs bosons production in the minimal supersymmetric (SUSY) model at a high energy $e^{+} e^{-}$collider, we presented both analytic and numeric results for production cross sections as a function of the parameters of the minimal SUSY model, and assessed the discovery potential of such a machine as a function of the center of mass energy. The conclusion was that a $500 \mathrm{GeV} e^{+} e^{-}$collider should produce and detect SUSY Higgs bosons over a large domain of parameter space.

\section{Ongoing \& Proposed Research:}

The ultimate objective of any such project is to identify characteristic signatures for each model which can be used to either discover the applicability of the model, or to prove its irrelevance. We intend to expand the scope of our study to include a larger class of models, and to update the constraints on the parameter space as new data becomes available. Presently, there exist many apparent discrepancies between theory and experiment; the goal of our work is to maximize the precision of the comparisons to determine whether differences are a result of inherent uncertainties, or a signature of physics beyond the Standard Model.

\subsection{CTEQ Collaboration Activities:}

(Olness)

\section{INTRODUCTION \& PAST RESEARCH:}

The CTEQ (Collaborative Theoretical and Experimental Stuies of QCD) collaboration is comprised of 17 theorists and experimentalists who are pooling expertise and resources on common objectives that will contribute both to their individual projects and to the whole community. This initiative evolved naturally from collaborative work on QCD phenomenology, and concentrates on tasks which demand a combined effort of theorists and experimentalists.

Research topics include: global QCD analysis and extraction of parton distributions; dimuon production in deep-inelastic scattering and the strange-quark distribution; direct photon production; W- and Z-production; jet cross sections; heavy flavor production; higher-order calculations and resummations; higher-twist effects; and spin effects. Many of these topics overlap the research area described above. 
Current members are: Raymond Brock (Mich. St. U.), John Collins (Penn. St. U.), Joey Huston (Mich. St. U.), Steve Kuhlmann (Argonne Nat. Lab), Sanjib Mishra (Harvard U.), Jorge Morfin (Fermilab), Fredrick Olness (SMU), Joseph Owens (Florida St. U.), Jonathan Pumplin (Mich. St. U.), Jian-Wei Qiu (Iowa State), Davison Soper (U. of Oregon), Jack Smith (SUNY-Stony Brook), George Sterman (SUNY-Stony Brook), Wu-Ki Tung (Mich. St. U.), Harry Weerts (Mich. St. U.), James Whitmore (Penn. St. U.), and C.P. Yuan, (Mich. St. U.).

In addition, CTEQ hosted summer schools on QCD Analysis and Phenomenology in 1992, 1993, and 1994. These schools serve not only as a forum for the students to understand the salient points of QCD, but also for the CTEQ members and lecturers to interact and to collaborate on projects. (T. Sjöstrand and Z. Kunszt were invited lecturers for the 1993 school, and C. Bernard, G. Blazey, and B. Grinstein for 1994.) Additionally, CTEQ has released a Handbook of Perturbative QCD: Version 1.0, which will appear in Reviews of Modern Physics. ${ }^{[52]}$ This has proven to be a valuable resource for both students and practitioners.

CTEQ also sponsored a workshop, QCD at $2 \mathrm{TeV}$, that identified processes to be measured by the CDF and D0 detectors in the current Tevatron collider run that will contribute to a better understanding of QCD. Topics for discussion included: rapidity gap physics, using the large rapidity coverage of the detectors to its full extent $(|y|<3.5)$, measuring gluon distributions to $x=0.001$, internal structure of jets, comparison of jets at LEP and Tevatron, diffractive physics with high $p_{t}$ detectors, etc. This conference was successful, and workshops on related topics are planned for the future.

ONGOING \& PROPOSED RESEARCH:

The CTEQ summer schools and collaboration meetings have been effective in initiating cooperative projects. As one example, we have pooled our resources to compare, contrast, and extend the calculations of M. Aivazis, F. Olness, J. Collins, and W. Tung, and the complementary calculation of E. Laenen, S. Riemersma, J. Smith, and W.L. van Neerven. ${ }^{[?]}$ This collaboration gives us a unique opportunity to explore the theoretical stability of each calculation through the renormalization scale dependence, and make a precise estimate of the theoretical uncertainty in separate kinematic regimes. A first publication has appeared as a CTEQ preprint, ${ }^{[14]}$ and subsequent work is in progress.

The CTEQ summer schools have successfully exposed advanced graduate students and beginning postdocs to the current issues in QCD, and we are planning to continue this series. In collaboration with DESY, preparations are being made to hold the 1995 CTEQ school in Germany. Additionally, QCD will be the topic of the 1995 TASI school, and elements of the CTEQ summer school will be incorporated into this series. Although CTEQ was founded with TNRLC ${ }^{\star}$ funding, the collaboration has been sufficiently productive that the activities and projects are continuing without this support. The 1994 CTEQ school was sponsored by Argonne, Brookhaven, DESY, DOE, Fermilab, International Institute of Theoretical and Applied Physics, and NSF. The 1995 schools will be supported in part by DESY and TASI.

* Texas National Research Laboratory Commission. 


\subsection{References:}

[1] M.A.G. Aivazis, F. I. Olness, and Wu-Ki Tung. Physical Review Letters 65, 2339 (1990).

[2] M.A.G. Aivazis, F. I. Olness, and Wu-Ki Tung. Physical Review D50, 3085 (1994).

[3] M.A.G. Aivazis, J. C. Collins, F. I. Olness, and Wu-Ki Tung. Physical Review D50, 3102 (1994).

[4] Joseph F. Owens and Wu-Ki Tung, Ann. Rev. Nucl. Part. Sci. (1992).

[5] James Botts, et al., Phys. Lett. B304, 159 (1993).

[6] A. Bazarko, et al, (CCFR Collaboration), Determination of the Strange Quark Content of the Nucleon from a Next-to-Leading Order QCD Analysis of Neutrino Charm Production, Nevis report NEVIS-1502, June (1994).

[7] J. Botts, et al, Version 2 CTEQ Distribution Functions in Parametrized Form, MSU preprint in preparation.

[8] P. Nason, S. Dawson and R.K. Ellis, Nucl. Phys. B303, 607 (1988); B327, 49 (1989); (E).B335, 260 (1990);

[9] E. Laenen, S. Riemersma, J. Smith, and W.L. van Neerven, Nucl. Phys. B392, 162 (1993); ibid., 229 (1993).

[10] T. Gottschalk, Phys. Rev. D23, 56 (1981).

G. Ingelman and G.A. Schuler, Z. Phys. C40, 299 (1988);

Gerhard A. Schuler, Nucl. Phys. B299, 21 (1988).

A. Ali, F. Barreiro, J.F. de Troconiz, G.A. Schuler, and J.J. van der Bij, Workshop on Large Hadron Colliders, Aachen, Germany, Oct 4-9, 1990. CERN report 90-10, p.917 (1990);

J.J. van der Bij and G.J. van Oldenborgh, Z. Phys. C51, 477 (1991).

G. Kramer and B. Lampe, Z. Phys. C54, 139 (1992).

M. Glück, E. Reya and M. Stratmann, Nucl. Phys. B422, 37 (1994).

[11] J. Collins, F. Wilczek, and A. Zee, Phys. Rev. D18, 242 (1978).

[12] W.-K. Tung, Nucl. Phys. B315, 378 (1989).

[13] Heavy Quark Production in Deep Inelastic Scattering at HERA, P. Agrawal, F. Olness, S. Riemersma, and Wu-Ki Tung. SMU preprint in preparation.

[14] Leptoproduction of Heavy Quarks in the Fixed and Variable Flavor Schemes, F. I. Olness and S. Riemersma. SMU preprint SMU-HEP/94-21, August (1994).

[15] Leptoproduction of Heavy Quarks in the Fixed and Variable Flavor Schemes, F. I. Olness and S. Riemersma. Presentation by F. Olness at Particles \& Fields '94: Meeting of the Division of Particles \& Fields of the APS, Albuquerque, NM, 2-6 August 1994. SMU preprint SMU-HEP/94-22, August (1994).

[16] C.L. Basham, L.S. Brown, S.D. Ellis, and S.T. Love, Phys. Rev. Lett. 41 (1978) 1585; Phys. Rev. D19 (1979) 2018.

[17] R.D. Peccei and R. Ruckl, Nucl. Phys. B162 (1980) 125.

M. Dechantsreiter, F. Halzen, and D.M. Scott, Zeit. Phys. 8 (1981) 85.

[18] Dirk Graudenz, Hamburg University doctoral thesis, preprint DESY-T-90-01. 
[19] R. Meng, F. Olness, and D. Soper. Nuclear Physics B371, 79 (1992).

[20] J. C. Collins and D. E. Soper, Nucl. Phys. B284 (1987) 253.

[21] C.T.H. Davies, B.R. Webber, and W.J. Stirling, Nucl. Phys. B256, 413 (1985).

[22] E.L. Berger and J. Qiu, Phys. Rev. D44, 2002 (1991);

J.C. Collins and R.K. Ellis, Nucl. Phys. B360, 3 (1991).

[23] G. Altarelli, R.K. Ellis, M. Greco, and G. Martinelli, Nucl. Phys. B246 (1984) 12. P. Arnold, R.K. Ellis, M.H. Reno Phys. Rev. D40, 912, (1989);

Peter B. Arnold, M.Hall Reno, Nucl. Phys. B319 (1989) 37; Erratum-ibid., B330 (1990) 284.

Peter B. Arnold and Russel P. Kauffman, Nucl. Phys. B349, (1991) 381.

[24] P.B. Arnold and R.P. Kauffman, Nucl. Phys. B349, 381 (1991).

[25] J.G. Korner, E. Mirkes, G.A. Schuler, Int. J. Mod. Phys. A4, 1781 (1989); R.S. Fletcher, F. Halzen, S. Keller, W.H. Smith, Phys. Lett. B266, 183 (1991); J. Chay, S. D. Ellis, W. James Stirling, U. of Washington preprint DOE-ER-4042328, May 1991.

[26] J. Botts and G. Sterman, Phys. Lett. B224, 201 (1989); Erratum, ibid., B227, 501 (1989).

[27] J. Collins, D. Soper and G. Sterman, Nucl. Phys., B263, 37 (1986);

John C. Collins, D. E. Soper, G. Sterman, Perturbative QCD, A.H. Mueller, ed., World Scientific Publ., 1989.

[28] Semi-Inclusive Deeply Inelastic Scattering at Electron-Proton Colliders: From Small to Large $P_{T}$, R. Meng, F. Olness, and D. Soper. SMU preprint SMU-HEP/94-13, in preparation.

[29] E. Eichten, I. Hinchliffe, K. Lane, C. Quigg, Rev. Mod. Phys. 56, 579 (1984);

M.R. Barnett, I. Hinchliffe, J.F. Gunion, B. Hubbard, H.E. Haber, H. Trost, Argonne preprint ANL-HEP-TR-90-86, Nov. 1990;

J.F. Gunion, G.L. Kane, V. Barger, R.M. Barnett, G. Bhattacharya, K. Cheung, D. Dicus, H.E. Haber, T. Han, I. Hinchliffe, B. Hubbard, R. Kauffman, B.A. Kniehl, G. Ladinsky, R.J.N. Phillips, H.J. Trost, R. Vega, C.P. Yuan, UC-Davis preprint 91-0010, Jan. 1991;

V. Barger, K. Cheung, T. Han, J. Ohnemus, D. Zeppenfeld, Phys. Rev. D44, 1426 (1991).

[30] M.S. Chanowitz, Ann. Rev. Nucl. Part. Phys. 38, 323 (1988);

R.N. Cahn and M. Suzuk, Phys. Rev. Lett. 67, 169 (1991).

[31] The Higgs Hunter's Guide. J. Gunion, H. Haber, G. Kane, S. Dawson.

[32] D. A. Dicus and V. S. Mathur, Phys. Rev. D7, 3111 (1973);

M. Veltman, Acta Phys. Pol. B8, 475 (1977);

B.W. Lee, C. Quigg, and H. B. Thacker, Phys. Rev. Lett. 38, 883 (1977); Phys.

Rev. D 16, 1519 (1977).

W. Marciano, G. Valencia, and S. Willenbrock, Phys. Rev. D 40, 1725 (1989).

[33] M. E. Ebel and F. Olness, Physical Review D30, 1034 (1984).

[34] F. Olness and M. E. Ebel, Physical Review D32, 1769 (1985). 
[35] J. F. Gunion, B. Kayser, R. N. Mohapatra, N. G. Deshpande, J. Grifols, A. Mendez, F. Olness, and P. B. Pal. 1986 Summer Study at Snowmass, Colorado;

Proc. of 1986 Summer Study on the Physics of the Superconducting Supercollider, (ed., R. Donaldson and J. Marx), p. 197 (1987).

[36] J. C. Collins and F. Olness,

Physics Letters B187, 376 (1987).

[37] J. Gunion, A. Mendez, and F. Olness.

Int. Journal of Modern Physics A2, 1085 (1987).

[38] W. Smith, D. Atwood, A. Caldwell, F. Cornet, G. Couture, M. Drees, W. Frisken, C. Heusch, J. Hewett, B. Irwin, U. Mallik, F. Olness, D. Reeder, T. Rizzo, J. Robinson, K. Sugano, J. Woodside, and D. Zeppenfeld. Proceedings of the Summer Study on High Energy Physics in the 1990s, June 27-July 15, 1988, Snowmass, Colo.;

High Energy Physics in the 1990s, (ed., S. Jensen), p. 195 (1989).

[39] J. Gunion, J. Grifols, A. Mendez, B. Kayser, and F. Olness.

Physical Review D40, 1546 (1989).

[40] F. Olness, in Proc. of XII Warsaw Symp. on Elementary Particle Physics, Kazimierz, Poland, May 29 - Jun 2, 1989;

Frontiers in Particle Physics, (ed., Z. Ajduk, et. al), p. 602 (1990).

[41] D. Atwood, U. Baur, J. Blümlein, G. Couture, M. A. Doncheski, R. Fletcher, D. Goddard, S. Godfrey, J. L. Hewett, D. P. Johnson, S. Keller, B. A. Kniehl, G. Levman, R. Lewis, L. Marleau, S. Mani, R. Meng, F. Olness, R. Orr, D. Reeder, T. G. Rizzo, G. Schuler, W. H. Smith, H. Spiesberger, K. Sugano, and R. Talaga.

Proceedings of the 1990 DPF Summer Study on High Energy Physics: Research Directions for the Decade, Snowmass, CO, p. 531, (1992).

[42] G. Senjanović and R. N. Mohapatra, Phys. Rev. D12, 1502 (1975);

E. Ma, Phys. Rev. Lett. 59, 607, (1987);

[43] H. Harari and Y. Nir, Nuc. Phys. B292, 251 (1987);

[44] X.-G. He, B. McKellar, and S. Pakvasa, Phys. Rev. Lett. 61, 1267 (1988);

[45] P. Langacker and S. U. Sankar, Phys. Rev. D 40, 1596 (1989);

[46] N.G. Deshpande, J.F. Gunion, B. Kayser, and F. Olness

Physical Review 44, 837 (1991).

[47] J. Gunion, L. Roszkowski, A. Turski, H. Haber, G. Gamberini, B. Kayser, S. Novaes, F. Olness, and J. Wudka.

Physical Review D38, 3444 (1988).

[48] J. Ellis, G. Ridolfi, and F. Zwirner, Phys. Lett. B257, 83 (1991).

[49] H.E. Haber and R. Hempfling, Phys. Rev. Lett. 66, 1815 (1991).

[50] A. Brignole, J. Ellis, J.F. Gunion, M. Guzzo, F. Olness, G. Ridolfi, L. Roszkowski, and F. Zwirner. Proceedings of the Workshop: $e^{+} e^{-}$Linear Colliders at $500 \mathrm{GeV}$ : the Physics Potential, Ed. P.M. Zerwas, p.613, (1992).

[51] P. W. Johnson, F. Olness, and W.-K. Tung.

Physical Review D36, 291 (1987).

[52] Handbook of Perturbative QCD: Version 1.0, Raymond Brock, John Collins, Joey Huston, Steve Kuhlmann, Sanjib Mishra, Jorge Morfin, Fredrick Olness, Joseph 
Owens, Jonathan Pumplin, Jian-Wei Qiu, Davison Soper, Jack Smith, George Sterman, Wu-Ki Tung, Harry Weerts, James Whitmore, C.P. Yuan, (CTEQ Collaboration). Fermilab-Pub-93-094, April (1993). To appear in Reviews of Modern Physics. 


\section{4) Roberto Vega:}

\section{Progress Report and Proposal}

\subsection{Overview:}

My research activities have predominantly focused on the phenomenology of electroweak symmetry breaking (EWSB) at future colliders.

Despite the remarkable successes of the Standard Model (SM) the mechanism by which electroweak symmetry is broken (EWSB) remains a mystery. The only certainty is that evidence for the mechanism of EWSB must manifest itself below the $1 \mathrm{TeV}$ energy scale. ${ }^{[1,2,3]}$ The Superconducting Supercollider (SSC) was in part being built to explore this realm of particle physics. We now hope to achieve the same goals using the Large Hadron Collider (LHC) to be built at CERN.

Much theoretical effort has gone into trying to understand the mechanism by which electroweak symmetry is broken. ${ }^{[4]}$ The phenomenological implications of the most promising theoretical models have been studied extensively. ${ }^{[4,6]}$ While these studies indicate that prospects for Higgs discovery at the $\mathrm{LHC}$ are excellent for most of the allowed parameter space, ${ }^{[4,7]}$ it is still not certain that a Higgs particle, or other evidence for EWSB, will be discovered. The reason for this follows.

There are two scenarios which will be particularly difficult for Higgs exploration. In one scenario fundamental Higgs scalars exist and nature is supersymmetric. In this case one expects Higgs scalars with masses of about $100 \mathrm{GeV}$. We will see below that we are now optimistic that with the LHC we will be able to effectively explore this possibility. In the second scenario electroweak symmetry is broken dynamically. In this case there is a second threshold ${ }^{[1]}$ for the weak interactions beyond which the interactions among longitudinal gauge bosons become strong. ${ }^{[1,2,3]}$ This threshold is reached at interaction energies of about $1 \mathrm{TeV}^{[1,2,3]}$ Beyond this threshold vector boson scatterings can exhibit resonances. These resonances, however, may be beyond the energy reach of the LHC. As it turns out, among the large number of possibilities for EWSB these two are the theoretically favored scenarios, ${ }^{[4,8]}$ they are sometimes referred to as worst case scenarios. Before we can ensure that these two scenarios can be adequately explored by the LHC a great deal of the more detailed technical work remains to be completed. It is with this purpose in mind that several of the projects listed below are proposed. Several of the projects deal with ways of increasing the sensitivity of the LHC detectors to any enhancement in the scattering of longitudinally-polarized vector bosons, while others deal with new mechanisms for searching for a Higgs with mass in the regime $80<m_{H}<120$ $\mathrm{GeV}$.

There are other scattered topics discussed below which do not fall neatly under one single heading. The list includes the phenomenology of models with Higgs triplet representations, models with CP violation in the Higgs sector, Tau decays, and Electroweak Effective Lagrangians.

What follows is a topic by topic summary of my past contributions and my future research plans. Although an attempt has been made to provide some references, they are by no means complete. The only purpose in providing them is to give some rough sense of how my work fits into the enormous bodies of literature on the subjects addressed. 


\subsection{Strongly Interacting Gauge Bosons:}

\section{INTRODUCTION AND PAST RESEARCH:}

The phenomenology of strongly interacting gauge bosons essentially boils down to distinguishing between the scattering of longitudinal- and transverse-polarized vector bosons at the LHC. If electroweak symmetry is broken dynamically the expected resonances in gauge boson scattering may occur at energies beyond the reach of the LHC. In that case the signal for EWSB would be a slight enhancement in the scattering of longitudinally-polarized vector bosons. ${ }^{[9]}$ Only if one can distinguish longitudinal- from transverse-polarized vector bosons will this enhancement be observable, and only then can one guarantee that the LHC will uncover the mystery of the mechanism of EWSB .

In collaboration with D.A. Dicus of the University of Texas at Austin we developed techniques to evaluate the exact matrix elements for $f f \rightarrow f f+W W^{[11,12,13]}$ The evaluation of the amplitude for this process involved a large number of Feynman diagrams. This was the first exact calculation of the cross section for vector boson scattering in $p p$ collisions. Many papers using techniques similar to ours later confirmed our results (see for example Refs. 14 and 15).

Our results dispelled the incorrect belief that the scattering of transverse-polarized vector bosons would be negligible at future hadron colliders. ${ }^{[13]}$ They had a major bearing on detector design considerations and stimulated other studies to determine detector requirements for investigating longitudinal-polarized vector boson scattering at future hadron colliders!

\section{ONgoing AND Proposed Research:}

- It is proposed to continue ongoing work to find experimentally implementable cuts to distinguish longitudinal- from transverse-polarized vector boson scattering. Three separate tasks will be completed for this purpose. First, the yield of $W$-pairs from $\gamma \gamma$ and $\gamma W$ fusion in $p p$ collisions will be accurately determined. My preliminary estimates indicate these processes may be the dominant electroweak background in heavy Higgs searches. The second and third tasks involve the use of two different signal enhancement strategies designed to facilitate the discovery of a heavy Higgs, or of an enhancement in the scattering of longitudinally-polarized vector bosons. One of these strategies was developed in the process of comparing the event structure of signal and backgrounds for longitudinallypolarized like-sign $W W$ scattering. ${ }^{[13]}$ (The other signal enhancing strategy that will be explored involves the use of rapidity gap signatures, and is discussed separately in the next section.)

The work on strongly interacting gauge bosons will be in collaboration with D.A. Dicus (UT, Austin) and J.F. Gunion (UC, Davis). 


\subsection{WW Scattering and Rapidity Gaps:}

InTRODUCTION AND PAST RESEARCH:

An alternate approach for enhancing the longitudinal to transverse ratio may be the rapidity gap signature.

In several of the Snowmass studies ${ }^{[6]}$ it has been pointed out that the color structure of events associated with the production of a SM Higgs via $W W$ fusion is quite different from that of the associated QCD backgrounds. This is intuitively obvious because the $W$ 's involved in Higgs production and decay are color singlets. The QCD backgrounds, on the other hand, involve multi-gluon emissions which may or may not be in color singlets. Therefore, it seems likely that the overall multiplicity associated with Higgs production will be relatively low when compared to the QCD backgrounds.

Motivated by this possibility, J.D. Bjorken ${ }^{[19]}$ has suggested that the event structure associated with Higgs production may yield events with large rapidity gaps. The reasoning is that Higgs production via $W$-fusion is basically a double diffraction process which would lead to two jets (the spectator jets) in the high rapidity regions $(|y|>5)$ and four central jets, coming from the decay products of the Higgs with $|y|<2.5$. Since the $W$ 's do not carry color there is no color exchange between the colliding beams and hence there are no final state interactions between the beams that would tend to wash out the gap. This would result in a relatively quiet (low multiplicity) event. ${ }^{[19]}$ Recently I have found that at the parton level the event structure suggested by Bjorken actually occurs more than $80 \%$ of the time in those events associated with Higgs production.

\section{Ongoing and Proposed Research:}

If it survives, the rapidity gap signature would be a very efficient way of extracting a heavy Higgs signal from the QCD backgrounds. Several studies have concentrated on determining how well the QCD backgrounds can be suppressed by this technique..$^{[20,21]}$ However, the efficiency of this technique in suppressing backgrounds from transverse $W$ scattering has not been studied.

We propose here to study how well the rapidity gap signature can be used to distinguish longitudinal from transverse-polarized vector boson scattering.

The work on rapidity gaps will be in collaboration with J.D. Bjorken and Gary Greenbaum (SLAC).

\subsection{Searches for Intermediate Mass Higgs at the LHC: INTRODUCTION AND PAST RESEARCH:}

If nature is supersymmetric it is very likely that fundamental Higgs scalars with a mass of the order of $M_{Z}$ exist. Experiments at the LHC must be geared to cover properly the mass regime $80<M_{H}<100 \mathrm{GeV}$. If we cannot study this mass regime we will be blind to, what is considered by many experts, the most likely region of parameter space for Higgs masses.

Recently it had been clearly established that a machine such as the LHC would allow exploration of most of the supersymmetric parameter space. ${ }^{[4,7]}$ However, there remained a controversy over whether the signal for a Higgs with mass $80<M_{H}<100 \mathrm{GeV}$ could be extracted from the overwhelming backgrounds present at the LHC. ${ }^{[4,7]}$ In this mass regime 
the dominant decay mode of the Higgs is into a $b \bar{b}$ pair, backgrounds from QCD and $Z$ decay would be overwhelming. ${ }^{[4,22]}$ Thus in this mass regime we were forced to consider the rare decay mode $H \rightarrow \gamma \gamma$. As suggested by W.J. Marciano ${ }^{[23]}$ and independently by J.F. Gunion ${ }^{[24]}$ the process $g g \rightarrow t \bar{t} H \rightarrow \gamma \gamma+l \nu+X$ provided the most promising way to survey this Higgs mass regime. Although this process is suppressed by a small $H \rightarrow \gamma \gamma$ branching ratio (of the order of $10^{-3}$ ), it is enhanced by the large gluon luminosity and the large $h t \bar{t}$ coupling. For this search strategy to work, detectors must have a photonphoton resolution of $\pm 3 \mathrm{GeV}$ and a good jet- $\gamma$ discrimination of $R_{j \gamma} \sim 3-5 \times 10^{-4}$. $^{\text {[25] }}$ While these requirements may be achievable by general purpose detectors at the LHC it seemed imperative to continue to search for alternate mechanisms to improve our chances for Higgs discovery in this mass regime.

Dai, Gunion, and I have recently proposed a scheme, which makes use of the dominant decay mode $h \rightarrow b \bar{b}$, that would allow SUSY-Higgs discovery over most of the SUSY parameter space and, most importantly, over the difficult region: $85<m_{H}<100 \mathrm{GeV}$ and $\tan \beta>5 .^{[27]}$ This work established that, at the LHC, discovery of at least one of the Higgs bosons of the minimal SUSY model is guaranteed over the entire range of parameter space.

\section{Ongoing AND Proposed RESEARCH:}

Ongoing work in the intermediate mass region involves the study of the process $g g \rightarrow$ $b \bar{b} h$. Our preliminary results show that discovery of two neutral Higgs bosons of the minimal supersymmetric model will be possible at the LHC in the $b \bar{b} b \bar{b}$ final state. These results will complement those obtained in ref. 27.

We hope to continue to further develop the intermediate search strategies that have been developed so far. This process of refinement must continue until the LHC is functional.

The work on intermediate-mass-Higgs searches will be in collaboration with J.F Gunion (UC, Davis) and Jin Dai (UC, San Diego).

\subsection{Models with Triplet Representations in the Higgs Sector:}

\section{INTRODUCTION AND PAST RESEARCH:}

While a single Higgs doublet provides the most economical mechanism for EWSB in the Standard Model there are no compelling reasons (other than simplicity and questions of naturalness) why higher Higgs multiplets cannot participate in EWSB ${ }^{[4]}$ For example, models with Higgs triplets have been proposed as a means to generate Majorana masses for the neutrinos. ${ }^{[30]}$ Usually the problem with these models is that they violate the tree level relation $\rho=1$. However, $\mathrm{H}$. Georgi has managed to construct a model in which Higgs triplets participate in EWSB and yet the tree level $\rho=1$ relation is preserved. ${ }^{[29]}$ The most general Higgs potential which preserves the custodial $S U(2)$ symmetry was constructed by Chanowitz and Golden. ${ }^{[31]}$

In collaboration with J.F. Gunion and J. Wudka we have studied the consistency of these models. ${ }^{[33]}$ We found that such models require fine tuning to avoid large radiative corrections to the relation $\rho=1$. In fact, if one requires $\rho=1$ at tree level, the one loop corrections to this relation are infinite. 
We also studied the phenomenology of such models at presently operating $e^{+} e^{-}$colliders. We mainly concentrated on the fermionic decay modes of the Higgs bosons. ${ }^{[32]}$

\section{ONGOING AND PROPOSED RESEARCH:}

The objective of this project is to study the phenomenology of these models at the Next Linear Collider (NLC). I would like to study production mechanisms, decay modes and lifetimes of the Higgs triplets in the Georgi model. This would also involve studying the associated backgrounds. One process which would be studied is charged Higgs production and decay via the process $e^{+} e^{-} \rightarrow W^{+} Z+e^{-} \nu$. This process would be of use in the study of EWSB at future $e^{+} e^{-}$colliders whether or not there exist charged Higgs bosons with $W Z H$ couplings.

The work on triplet Higgs wiil be in collaboration with J.F. Gunion (UC, Davis)

\section{6 t $\overline{\mathrm{t}}$ Phenomenology and CP Violation Effects}

InTRODUCTION AND PAST RESEARCH:

Future high energy colliders may be able to settle other unanswered questions related to EWSB. One of these is whether CP is also violated when the electroweak symmetry is broken. In fact, in models with several doublets of fundamental scalars Weinberg has shown that it is natural for CP to be violated maximally ${ }^{[34]}$ It would be interesting to determine if Weinberg's statements generalize to other exotic representations of $S U(2)$, and if so, to map out the experimental consequences for this kind of physics for the LHC.

Perhaps the simplest manifestation of this kind of $\mathrm{CP}$ violation is in the electron dipole moment $\left(d_{e}\right)$. Barr and Zee were the first to obtain an estimate for $d_{e} e^{[36]}$. However, they only considered the contributions from the top quark loop. In collaboration with Gunion we considered the complete gauge invariant set of two loop diagrams which contribute to $d_{e}$ in the Weinberg model and found that, $d_{e} \sim 10^{-26} \mathrm{e}-\mathrm{cm}$, a value close to the present experimental bounds. In a more recent paper with Pomarol we showed explicitly the connection between CP nonconservation and the breaking of custodial SU(2) in the Higgs sector. ${ }^{[37]}$ In that work we attempted to place a limit on the $\mathrm{CP}$ violating parameters by using the experimental limits on $\Delta \rho$.

\section{ONGOING AND PROPOSED RESEARCH:}

One possible way to explore CP violation in the Higgs sector is to take advantage of the large $h t \bar{t}$ coupling. It is proposed here to use the process $g g \rightarrow h t \bar{t}$ discussed in ref. 26 to identify possible CP violating observables. We have found ${ }^{[26]}$ that the rates for this process, may be sufficient to study the CP properties of the $h t \bar{t}$ vertex. While previous studies have considered loop induced CP nonconservation in this vertex ${ }^{[38]}$ the present process has the advantage that it is at tree level. I propose a continuation of the work of ref. 26 seeking correlations sensitive to the CP nature of the $h t \bar{t}$ vertex. This would entail modifying the codes developed there to reflect the new $\mathrm{CP}$ violating $h t \bar{t}$ coupling.

The work on CP violation will be in collaboration with J. Dai (UC, San Diego). 


\subsection{Tau Decays to Three Leptons:}

The CLEO collaboration at Cornell may have already accumulated enough $\tau$ events to allow measurement of the branching ratios for the four leptonic final states, $e^{+} e^{+} e^{-} \nu_{e} \bar{\nu}_{\tau}$, $\mu^{+} e^{+} e^{-} \nu_{\mu} \bar{\nu}_{\tau}, e^{+} \mu^{+} \mu^{-} \nu_{e} \bar{\nu}_{\tau}$ and $\mu^{+} \mu^{+} \mu^{-} \nu_{\mu} \bar{\nu}_{\tau}$; or to place limits on the branching ratios for the corresponding lepton number violating processes which do not involve neutrinos in the final states. ${ }^{[39]}$ In order to carry out their analysis they need the theoretical predictions of the rates for these processes.

Thanks to the presence of members from the CLEO collaboration in our group here at SMU I became interested in $\tau$-physics. In collaboration with D.A. Dicus we have recently completed ${ }^{\left[{ }^{(0)}\right.}$ the calculation of the standard model predictions for the rates for $\tau \rightarrow l l \bar{l} \nu \bar{\nu}$ and $\tau \rightarrow \gamma l \nu \bar{\nu}$. We found the interesting result that there are strong angular correlations between the momenta of the final state leptons. The results have a major bearing on the experimental techniques that are used to discover or place limits on these decay modes.

While at the moment I do not foresee further work in this area, I am becoming increasingly interested in the physics of the heaviest of the known leptons.

\subsection{A New Helicity Method for Massive Particles:}

The complexity and large number of Feynman diagrams involved in many of the processes considered for study at future colliders required the development of techniques for evaluating Feynman diagrams at the amplitude level. ${ }^{[1]}$ Unfortunately, the beautiful techniques developed ${ }^{[41]}$ are adequate only for processes involving massless quarks. To handle complicated processes involving the top quark one needs to develop a new approach.

I am in the process of finishing work on a new approach to calculate helicity amplitudes for processes involving massive fermions. I use these methods to obtain relatively concise expressions for the helicity amplitudes of several complicated processes involving the top quark. ${ }^{[26,27]}$ I have tested the method on simple processes that have been previously considered in the literature and my results results agree exactly with the conventional approach.

I would like to extend this helicity formalism to particles of higher spin. This would be useful for the phenomenology of spin $3 / 2$ particles predicted by SUSY theories.

The work on the helicity method will be in collaboration with J. Wudka (UC, Riverside).

\subsection{The Process gg $\rightarrow$ t $\overline{t g g: ~}$}

Determining the rates for this process is extremely important because this process is a dominant background to many signals of new physics (see for example Ref. 42). The rates for this process have been estimated but an exact calculation has not been done. The reason of course is the complexity and enormous number of diagrams. We intend to use the helicity methods developed by me to compute these rates. Part of my motivation for this project is establishing professional relations with high energy physicists in Mexico. I hope that this project will serve as a training tool for one or two graduate students in Mexico.

The work on associated gluon production will be in collaboration with Lorenzo Diaz Cruz (Universidad Autonoma de Puebla, Mexico). 
4.10 Probing Tree Level Generated Operators in the Low Energy Electroweak Effective Lagrangian:

Effective Lagrangians can be used to parametrize the effects of physics beyond the standard model. Assuming the complete theory is a gauge theory, Arst, Einhorn, and Wudka ${ }^{[43]}$ have written down all the tree level generated operators in the Electroweak Effective Lagrangian. These operators may exhibit measurable effects at low energies. We propose to study these operators to identify low energy observables that can be used to constrain them. In this work I hope to make use of Hornbostel's expertise on Effective Lagrangians.

The work on Effective Lagrangians will be in collaboration with Bohdan Grzadkowski (Warsaw University) and Jose Wudka (UC, Riverside).

\section{REFERENCES}

1. D.A. Dicus and V.S. Mathur, Phys. Rev. D7 (1973) 3111.

2. B.W. Lee, C. Quigg, H.B. Thacker, Phys. Rev. D16 (1977) 1519; B.W. Lee, C. Quigg, H.B. Thacker, Phys. Rev. Lett. 38 (1977) 883.

3. M. Veltman, Acta Phys. Pol.B8 (1977) 475.

4. For a review and extensive list of references see J.F. Gunion, H.E. Haber,G.L. Kane, S. Dawson, The Higgs Hunter's Guide, (Addison Wesley, Redwood City, CA., 1990).

5. Y.L. Dokshitzer, V.A. Khoze, and S. Troyan, in Proceedings of the 6th International Conference in Collisions (1986), ed. M. Derrick (World Scientific, Singapore, 1987) p.365; S.J. Brodsky and J.F. Gunion, Phys. Rev. D37 (1976) 402.

6. R. Donaldson and J.G. Morfin, (Ed.), Design and Utilization of the Superconducting Super Collider, proceedings, New York, USA: Aip ( 1985) 851p. See also ref. 5.

7. See for example J.F. Gunion, G.L. Kane et al., 'Overview and Recent Progress in Higgs Boson Physics at the SSC', to appear in Proceedings of the 1990 Snowmass Summer Study on "Research Directions for the Decade", preprint PRINT-91-0010 (1990).

8. G.L. Kane, UM-TH-91-02, Feb 1991. 38pp. see also UM-TH-90-02, Feb 1990. 13pp.

9. M.S. Chanowitz and Mary K. Gaillard, Nucl. Phys. B261 (1985) 379.

10. M.S. Chanowitz, 'Probing Electroweak Symmetry Breaking at the SSC: A No Lose Corollary', in Proceedings of the DPF '87, 13th Annual Mtg. of Div. of Particles and Fields of the APS, Salt Lake City, Utah (1987).

11. D.A. Dicus, R. Vega, Phys. Rev. Lett. 57 (1986) 1110; A. Abbasabadi, W.W. Repko, Duane A. Dicus, Roberto Vega, Phys. Rev. D38 (1988) 2770.

12. D.A. Dicus, S.L. Wilson, and R. Vega, Phys. Lett. B192 (1987) 231.

13. R. Vega, D.A. Dicus, Nucl. Phys. B329 (1990) 533; D.A. Dicus, R. Vega, Phys. Lett. B217 (1989) 194.

14. A. Tofighi-Niaki and J.F. Gunion, Phys. Rev. D38 (1988) 1433. 
15. U. Baur and E.W.N. Glover, Phys. Lett. B252 (1990) 683; U. Baur and E.W.N. Glover, Nucl. Phys. B347 (1990) 12.

16. M.S. Berger and M.S. Chanowitz, Phys. Lett. B263 (1991) 509.

17. D.A. Dicus, J.F. Gunion, R. Vega, Phys. Lett. B258 (1991) 475; D.A. Dicus, J.F. Gunion, L.H. Orr, R. Vega, Nucl. Phys. B377 (1992) 31.

18. V. Barger, Kingman Cheung, T. Han, and R.J.N. Phillips, Phys. Rev. D42 (1990) 3052.

19. J.D. Bjorken, Int. J. of Mod. Phys.A7 (1992) 4189; J.D. Bjorken, Phys. Rev. D47 (1992) 101.

20. R.S. Fletcher and T. Stelzer, Phys. Rev. D48 (1993) 5162.

21. H. Chehime,M.B. Gay Ducati, A. Duff, A., F. Halzen, A.A. Natale, T. Selzer, and D. Zeppenfeld, Phys. Lett. B286 (1992) 397.

22. R.K. Ellis, I. Hinchliffe, M. Soldate and J.J. van der Bij, Nucl. Phys. B297 (1988) 221; J.F. Gunion, G.L. Kane and J. Wudka, Nucl. Phys. B299 (1988) 231.

23. W.J. Marciano and F.E. Paige, Phys. Rev. Lett. 66 (1991) 2433.

24. J.F. Gunion, Phys. Lett. B261 (1991) 510.

25. M. Mangano, SDC Collaboration Note SSC-SDC-90-00113.

26. J. Dai, J.F. Gunion, and R. Vega, Phys. Rev. Lett. 71 (1993) 2699.

27. J. Dai, J.F. Gunion, and R. Vega, Phys. Lett. B315 (1993) 355.

28. D.A. Dicus, J.F. Gunion, L.H. Orr, and R. Vega (SLAC), Nucl. Phys. B377 (1992) 31.

29. H. Georgi and M. Machacek, Nucl. Phys. B262 (1985) 463.

30. G.B. Gelmini, M. Roncadelli, Phys. Lett. B99 (1981) 411.

31. M.S. Chanowitz and M. Golden, Phys. Lett. B165 (1985) 10.

32. J.F. Gunion, R. Vega, J. Wudka, Phys. Rev. D42 (1990) 1673.

33. J.F. Gunion, R. Vega, J. Wudka, Phys. Rev. D43 (1991) 2322.

34. S. Weinberg, Phys. Rev. D42 (1990) 860.

35. J.F. Gunion, R. Vega, Phys. Lett. B251 (1990) 157.

36. S.M. Barr, A. Zee, Phys. Rev. Lett. 65 (1990) 21; ERRATUM-ibid. 65 (1990) 2920.

37. Alex Pomarol and R. Vega, Nucl. Phys. B413 (1994) 3.

38. C.R. Schmidt, M.E. Peskin, Phys. Rev. Lett. 69 (1992) 410; D. Chang and Wai-Yee Keung Phys. Lett. B305 (1993) 261; B. Grzadkowski, J.F. Gunion, Phys. Lett. B287 (1992) 237; J.M. Soares, CMU-HEP91-23 (1991) 5pp. (unpublished)

39. Ryszard Stroynowski, private communication.

40. D.A. Dicus and R. Vega, DOE-ER-40757-030 (1994) 13pp., to be published in Phys. Lett. B

41. R. Kleiss, W.J. Stirling, Nucl. Phys. B262 (1985) 235; K. Hagiwara, D. Zeppenfeld, Nucl. Phys. B274 (1986) 1; V. Barger, J. Ohnemus, R.J.N. Phillips, Phys. Rev. D35 (1987) 166; Zhan Xu, Da-Hua Zhang, Lee Chang, Nucl. Phys. B291 (1987) 392. 
42. R.M. Barnett, et.al., "Techniques for Finding Supersymmetry at the SSC", Berkeley SSC Workshop. (1987) 178; T. Garavaglia, Wai-kwok Kwong, Dan-Di Wu, Phys. Rev. D48 (1993) 1899.

43. C. Arzt, M.B. Einhorn, and J. Wudka, UM-TH-94-15 (1994) 37pp. 


\section{5) Benjamin Grinstein:}

\section{Progress Report and Proposal}

\subsection{Overview:}

During the short period in which I was at SMU I worked on several projects related to Heavy Quark Effective Theories.

In collaboration with $P$. Mende the form factors of the flavor changing vector current between a $\bar{q} Q$-meson and the lightest $\bar{q} q$ (pseudoscalar-)meson were computed exactly and explicitly in the 't Hooft model (planar QCD in $1+1$ dimensions) in the limit that the mass of $q$-quark vanishes[1,2,3]. It was proved that in the 't Hooft model, in the chiral limit, the form factors of flavor changing currents between ground state mesons (heavy-light to light-light) are given exactly by a single pole: exact pole dominance! In $3+1$ dimensions no similar statement could be made by use of our techniques, and work is in progress. This work opens up the possibility of computing from first principles the rate for semileptonic $B \rightarrow \pi$ decays. It is important to study the corrections from finite $N_{c}$ (number of colors) and non-vanishing $m_{q}$ (light quark mass).

Investigations of static and dynamical properties of heavy mesons in the 't Hooft model[1] have given light into issues of convergence of heavy quark expansions, and have been generally valuable in developing some intuitive understanding of non-perturbative phenomena of these hadrons. With Hornbostel, an expert on Quantum Chromo Dynamics in $1+1$ dimensions, the possibility of extending the preceding work to the finite number of colors domain is under study.

With G. Boyd a systematic investigation of the effects of simultaneous breaking of heavy quark and chiral symmetries in decay constants was made[4]. We derived the most general Lagrangian consistent with chiral, heavy quark, and strong interaction symmetries to order $1 / M$ and to linear order in the $S U(3)$ vector and axial currents. Two new dimensionful and five dimensionless couplings arise at this order. The heavy to light flavor changing current was derived to the same order, giving rise to two additional dimensionful constants and six dimensionless ones. The dimensionless parameters were shown to be irrelevant at $\mathcal{O}\left(\frac{1}{M}\right)$. The leading nonanalytic heavy quark and chiral symmetry violating corrections to heavy meson decay constants were computed, and implications to B decays were discussed; measurements of the experimentally accessible form factors for $D \rightarrow \pi l \nu$ and $B \rightarrow \pi l \nu$, along with knowledge of the $D^{*} D \pi$ coupling, determine the eight decay constants $f_{D}, f_{D_{s}}, f_{D^{*}}, f_{D_{s}^{*}}, f_{B}, f_{B_{s}}, f_{B^{*}}$ and $f_{B_{s}^{*}}$, as well as one relation among the $B \rightarrow \pi l \nu$ form factors, to $\mathcal{O}\left(\frac{1}{M}\right)$. The ratio $R_{1}=\frac{f_{B_{s}}}{f_{B}} / \frac{f_{D_{s}}}{f_{D}}$ was expressed in terms of two dimensionful couplings, and estimated to deviate from unity by no more than $10 \%$.

\subsection{References:}

[1] Heavy Mesons in Two Dimensions, P. Mende and B. Grinstein, Phys. Rev. Lett. 69 (1992) 1018.

[2] Exact Heavy to Light Meson Form-Factors in the Chiral Limit of Planar $(1+1)$ QCD, Benjamin Grinstein, SMU-HEP-94-11, May 1994. 9pp.

[3] Form factors in the heavy quark and chiral limit: Pole dominance in $\vec{B} \rightarrow \pi \bar{\nu}_{e}, \mathrm{~B}$. Grinstein and P.F. Mende, Nucl. Phys. B, in press

[4] Chiral and Heavy Quark Symmetry Violation in B Decays, C. Glenn Boyd, Benjamin Grinstein, SMU-HEP/9403, Feb. 1994, submitted for publ. to Nucl. Phys. B 


\section{6) Stephan Riemersma:}

Progress Report and Proposal

Calculating collider processes and comparing the calculations to data provide significant tests of our understanding of the theories that form the basis of particle physics. As detectors become more sophisticated and the amount of data increases, the uncertainties in the determination of the observables that measure our understanding of particle physics decrease. Experiments are now sufficiently precise that, to test the theories adequately, we need to calculate the radiative corrections to these collider processes.

\subsection{Heavy-Flavor Production:}

\section{(Riemersma)}

Motivation: Issues in heavy-flavor production have become increasingly important over the last several years. The solutions will rely upon further data acquisition at collider facilities and calculations with greater precision than the current ones. The examples are multifold. Recently CDF announced evidence indicating the existence of the top quark [1]. At this time, however, D0 is unable either to confirm or to deny the existence of the top quark. QCD predictions currently underestimate the measured cross section of CDF [2-4]. At this point, we await further data from $C D F$ and $D 0$, which will provide greater insight into the possible confrontation of experiment with theory.

Another source of contention is the controversy surrounding the bottom quark production cross section measurements of CDF and D0. While the D0 cross section exhibits agreement with the QCD predictions, the CDF result is larger by approximately a standard deviation [5]. With time, the CDF analysis has reduced the values of the data points but unless this continues, we may have a controversy not only pitting data against theory, but data against data.

With the $e^{+} e^{-}$B-factory to be built at SLAC, accurate heavy-quark production cross section calculations are mandatory for precision measurements of $B \bar{B}$ mixing, rare $B$ decays, and measurement of the CKM angles $\alpha, \beta$ and $\gamma[6]$. These phenomena are important tests of the Standard Model and unless the cross section predictions are sufficiently accurate, the uncertainties on the measurements will be dominated by the theoretical cross section uncertainty.

The high precision data of CCFR, NMC and EMC having reduced the uncertainty of the quark distributions in the proton [7-9], the greatest uncertainty remaining is the gluon distribution. Charm and bottom quark production at HERA should provide a constraint for the gluon distribution.

\section{ONGOING RESEARCH:}

E. Laenen (CERN), J. Smith (Stony Brook), W.L. van Neerven (Leiden) and I have calculated the complete $\mathcal{O}\left(\alpha_{s}\right)$ corrections to the virtual-photon initiated cross sections for heavy-flavor production in deeply inelastic electron-proton scattering [10]. The corrections are expressed in the form of structure functions $F_{2}\left(x, Q^{2}, m^{2}\right)$ and $F_{L}\left(x, Q^{2}, m^{2}\right)$. The results are used to predict the charm and bottom content of the proton and can be used to make a fit to the gluon distribution by working back from the data using the calculation.

We have also calculated the inclusive distributions of the heavy quark in rapidity and transverse momentum [11]. Calculating the distribution is particularly useful for the experimentalists because with this information, they can predict where the heavy-quark production events should occur in the detector. 
In collaboration with Fredrick Olness, we have just submitted a paper comparing two methods of calculating deep-inelastic heavy-quark production. One method assumes that in the lowest-order reaction, the virtual photon interacts with a gluon and produces a heavy-quark antiquark pair (flavor creation). This reaction occurs at $\mathcal{O}\left(\alpha_{s}\right)$ and the corrections have been calculated to $\mathcal{O}\left(\alpha_{s}^{2}\right)$. The second assumes the lowest-order reaction is the virtual photon exciting a heavy quark in the hadron (flavor excitation) to separate from the hadron and hadronize in the detector. This reaction occurs at $\mathcal{O}\left(\alpha_{s}^{0}\right)$ and the corrections have been calculated to $\mathcal{O}\left(\alpha_{s}\right)$. We have compared the flavor-creation calculation with the flavor-excitation result $[12,13]$. Specifically, we analyzed the mass factorization scale dependence of each calculation in the different regions of phase space and discussed the validity of both in the various regions.

PROPOSED RESEARCH:

The merging of the two calculations should provide an excellent tool for predicting heavy-quark production at HERA, by extending the perturbation series to three orders. This calculation is currently in progress. The LRSN calculation is applicable only for an incoming off-shell photon. Generalizing the calculation to include couplings to $W^{ \pm}$and $Z^{0}$ will improve calculations for initial state charged leptons and neutrinos interacting via an electroweak vector boson with a hadron to produce the heavy quark. This calculation is also underway. I am also engaged in fitting the $\mathcal{O}\left(\alpha_{s}^{2}\right)$ matrix elements with Smith and van Neerven by using a table of calculated values and an interpolating routine. Using the fit, we will be able to generate phenomenological results much more quickly than with the full matrix-element calculation. This project is very near completion.

\subsection{Photon Structure Functions:}

\section{(Riemersma)}

\section{INTRODUCTION:}

In the past two decades considerable interest has been generated in the study of photon-photon interactions in electron-positron colliders. When one photon is virtual and the other one is almost real, the analogy with deep-inelastic electron-nucleon scattering motivates introducing the corresponding structure functions $F_{k}^{\gamma}\left(x, Q^{2}\right)(k=2, L)$ for the photon.

Two-photon reactions are also important to understand as background processes to the normal $s$-channel reactions at present and future $e^{+} e^{-}$colliders. These machines will have a large amount of beamstrahlung $[14,15]$. Therefore a basic input is the parton density in a photon which will need to be modified if higher order perturbative QCD corrections are included.

\section{ONGOING RESEARCH:}

With many of the pieces of the calculation already available [16-20], E. Laenen (CERN), J. Smith (Stony Brook), W.L. van Neerven (Leiden) and I have completed NLO QCD analysis of the photon structure functions $F_{2}^{\gamma}\left(x, Q^{2}\right)$ and $F_{L}^{\gamma}\left(x, Q^{2}\right)$ for a real photon target [21], using the results of references [10,22]. In particular we studied the heavy-flavor content of the structure functions which is due to two different production mechanisms, namely collisions of a virtual photon with a real photon, and with a parton. We observed that the charm contributions are noticeable for $F_{2}^{\gamma}\left(x, Q^{2}\right)$ as well as $F_{L}^{\gamma}\left(x, Q^{2}\right)$ in the $x$-region studied. 


\section{PROPOSED RESEARCH:}

This work will be important for LEP-II given that the center of mass energy of the machine will be high and generate a large amount of beamstrahlung. Understanding the backgrounds from the two-photon physics will allow the search for new physics to proceed more vigorously. We are currently looking into the two-photon cross section at LEP-II.

\subsection{References:}

[1] The CDF Collaboration Fermilab-Pub-94/097-E, (Submitted to Physical Review).

[2] E. Laenen, J. Smith and W.L. van Neerven, Phys. Lett. 321B, 254 (1994); Nucl. Phys. B369, 543 (1992).

[3] P. Nason, S. Dawson and R.K. Ellis, Nucl. Phys B303, 607 (1988); ibid, B327, 49 (1989); erratum B335, 260 (1990).

[4] W. Beenakker, H. Kuijf, W.L. van Neerven and J. Smith, Phys. Rev. D40, 54 (1989); W. Beenakker, W.L. van Neerven, R. Meng, G.A. Schuler and J. Smith, Nucl. Phys. B351, 507 (1991).

[5] S. Riemersma and R. Meng, in Proceedings of the Workshop on B Physics at Hadron Accelerators, (Snowmass 1993) 333.

[6] See the Proceedings of the Workshop on B Physics at Hadron Accelerators (Snowmass 1993) for a comprehensive review and a complete list of references.

[7] The CCFR Collaboration Nevis R\#1502 June 1994; Phys. Rev. Lett. 70, 134 (1993).

[8] The EMC Collaboration, Nucl. Phys. B333, 1 (1990); Nucl. Phys. B321, 541 (1989).

[9] The NMC Collaboration, Nucl. Phys. A546, 1c-24c (1992); Phys. Lett. 249B, $366(1990)$

[10] E. Laenen, S. Riemersma, J. Smith, and W.L. van Neerven, Nucl. Phys. B392, 162 (1993).

[11] E. Laenen, S. Riemersma, J. Smith, and W.L. van Neerven, Nucl. Phys. B392, 229 (1993).

[12] M.A.G. Aivazis, F.I. Olness and W.-K. Tung, Phys. Rev. Lett. 65, 2339 (1990); M.A.G. Aivazis, F.I. Olness, and W.-K. Tung, Phys. Rev. D50, (1994); M.A.G. Aivazis, J.C. Collins, F.I. Olness and W.-K. Tung, Phys. Rev. D50, (1994).

[13] F. Olness and S. Riemersma, SMU HEP 94-21.

[14] M. Drees and R. Godbole, DESY-92-044; Phys. Rev. Lett. 67, 1189 (1991).

[15] O.J.P. Éboli, M.C. Gonzalez-Garcia, F. Halzen and S.F. Novaes, Phys. Rev. D47, 1889 (1993); M. Drees, M. Krämer, J. Zunft and P.M. Zerwas, Phys. Lett. 306B, 371 (1993).

[16] M. Glück and E. Reya, Phys. Rev. D28, 2749 (1983).

[17] M. Drees, M. Glück and E. Reya, Phys. Rev. D30, 2316 (1984).

[18] M. Glück, K. Grassie and E. Reya, Phys. Rev. D30, 1447 (1984).

[19] W.A. Bardeen and A.J. Buras, Phys. Rev. D20, 166 (1979); erratum D21, 2041 (1980). 
[20] D.W. Duke and J.F. Owens, Phys. Rev. D22, 2280 (1980).

[21] E. Laenen, S. Riemersma, J. Smith and W.L. van Neerven, Phys. Rev. D49, 5753 (1994).

[22] E.B. Zijlstra and W.L. van Neerven, Nucl. Phys. B383, 525 (1992); Phys. Lett. B273, 476 (1991); W.L. van Neerven and E.B. Zijlstra, Phys. Lett. B272, 127 (1991). 


\section{7) D. Rosenbaum and V. Teplitz:}

\section{Progress Report and Proposal}

\subsection{Non-Luminous Matter in Solar Orbit (Rosenbaum and Teplitz)}

INTRODUCTION \& PAST RESEARCH: In collaboration with D. Dicus at the University of Texas and workers at the Caltech Jet Propulsion Laboratory, we have, since 1987, been working on bounding (or detecting) non-luminous matter in orbit about the sun in a spherically symmetric distribution. We bound non-luminous matter by noting that such a distribution would increase the effective mass of the sun as seen by the outer planets and by finding the uncertainty in that effective mass for Uranus and Neptune. The limiting factor in our ability to make such bounds is the precision with which distances to the outer planets are known. These distance measurements are greatly improved when one of the Voyager space probes passes by the planet. In 1989 we published, in Astrophysical Journal, a bound of about an earth mass on non-luminous matter in solar orbit interior to Uranus; that work was based on data from the 1986 Voyager 2 flyby of Uranus. We have just submitted to Astophysical Journal our new results based on the extra information learned in the 1989 Voyager 2 Neptune flyby. We bound non-luminous matter in spherically symmetric orbit interior to Uranus to about a fifth of an earth mass, and interior to Neptune at about an earth mass.

Proposed Research \& ONgoIng Work: D. Rosenbaum and V. Teplitz, with D. Dicus at the University of Texas and with workers at the Caltech Jet Propulsion Laboratory, are continuing work on non-luminous matter in solar orbit. We are extending our earlier work to non-spherically symmetric distributions of non-luminous matter. This is an area of high current interest because: (a) modern comet theory raises a strong possibility that there is a belt of cometary material (Kuiper belt) just past Neptune where objects under a couple hundred kilometers cannot be seen with telescopes (comets are only 1-10 kilometers); and (b) Jewett and Luu in 1992 saw half a dozen $200 \mathrm{~km}$ objects at various distances past Neptune which could have been large objects in the Kuiper belt. We are adding to our investigation of planetary motion a parametrized Kuiper belt. We should be able either to detect a Kuiper belt if it is there at a level of a few tenths of an earth mass or to bound it at about that level.

\subsection{Unitary Higgs Amplitudes (Teplitz)}

Introduction \& PAST Research: With D. Dicus at the University of Texas, we looked at coupled channel W-W, Z-Z, and H-H scattering [3]. Dicus, in previous work, had looked at the results of constructing unitary amplitudes with the K-matrix and with Padé approximants. Our recent work uses the "N over D method" for constructing unitary coupled-channel amplitudes. In that method, solution of an integral equation yields an amplitude that obeys the unitarity condition exactly and that has a discontinuity across its left hand cut precisely equal to that of the input "potential". Our principal results were: (1) above a mass for an "elementary Higgs" in the 1-2 TeV range (depending on assumptions regarding the form of the potential), coupled channel scattering would give rise to a bound state in the Higgs channel below $2 m_{W} ;(2) \mathrm{W}-\mathrm{W}$ and Z-Z scattering amplitudes were calculated for several choices of the form of the potential and several values of 
an elementary Higgs mass; (3) within the N/D method, the width of an elementary Higgs is not greatly increased with increase in mass, contrary to results from other methods.

Proposed RESEARCH: We are currently exploring other possible calculations in the general area of Higgs Dynamics.

\subsection{References:}

[1] Bounds in Dark Matter in Solar Orbit, J.D. Anderson, D.A. Dicus, E.L. Lau, D. Rosenbaum, A.H. Taylor, V.L. Teplitz, Astrophysical Journal 342, 539 (1989).

[2] Improved Bounds on Non-Luminous Matter in Solar Orbit, J.D. Anderson, D.A. Dicus, T. Krisher, E.L. Lau, D. Rosenbaum, V.L. Teplitz, Submitted to Astrophysical Journal (1994).

[3] Coupled Channel Vector-Boson Scattering in the $N / D$ Method, D.A. Dicus and V.L. Teplitz, Physical Review D 49, 5735 (1994). 


\subsection{Asymptotic behavior of exclusive processes in QCD:} INTRODUCTION AND PAST RESEARCH:

I have been working on exclusive processes at high energies. In particular, the expression for the form-factors in terms of leading twist wave functions has been obtained and the factorization theorem has been proved[1]. These results were confirmed and expanded by several independent groups: Brodsky and Lepage[2], Duncan and Mueller[3], Efremov and Radyushkin[4], and Farrar and Jackson[5].

It is known that such formulae do not describe the experimental data with asymptoticlike wave functions. Our guess was that the correct wave functions have a much different behavior which is connected to nonperturbative large distance physics. Using QCD sum rules we found the quantitative properties of the corresponding nonperturbative wave function. Now these functions (Chernyak-Zhitnitsky (CZ) wave functions [6]) are well known in the literature.

\section{Proposed RESEARCH AND ONGOING WORK:}

At present, I am working on heavy-light quark meson systems and the SU(3) symmetry breaking effects in them that follow from QCD sum rules. It turns out that this system is very sensitive to QCD vacuum structure and very important questions, such as factorization properties of complex operators, can be answered from the analysis of these structures. Information on the vacuum properties of these systems can be used for the description of transverse quark distribution in mesons as well as for the analysis of QCD vacuum structure. It is known that the transverse quark distribution plays a key role in exclusive processes at intermediate momentum transfer. It turns out that the nontrivial transverse shape of the nonperturbative wave function can simulate the leading twist behavior at very modest momentum transfers[7].

\subsection{Axion Physics:}

\section{INTRODUCTION AND PAST RESEARCH:}

Since 1980 I have been working on the so-called strong CP violation problem and its relationship to the axion[8,9]. I introduced a model with an almost invisible axion[9]. In such model the $\mathrm{CP}$ violation problem in strong interactions is solved, but the axion is very difficult to observe. A priori there is no limit on the axion mass and coupling constant. However, a low mass axion with very small coupling constant is excluded by cosmological and astrophysical arguments. If the axion mass is of the order of $10^{-5} \mathrm{eV}$, axions may constitute the dark matter in the Universe. This model is referred to in the literature as the DFSZ (Dine-Fishler-Strednitsky-Zhitnitsky) $[9,10]$ axion model and there are several experimental groups searching for such axions.

PROPOSED RESEARCH AND ONGOING WORK:

Now I am working with R. Stroynowski on the theory of axions with analysis of possible search experiments which can be carried out to look for invisible axions[11]. In particular, we found the resonant mechanism for the axion-photon transition. It brings the experimentally accessible quantities within the reach of present day technology. 


\subsection{References:}

[1] Chernyak V. and A.Zhitnitsky, JETP Lett. 26,(1977),594.

[2] Brodsky S. and G.P.Lepage, Phys. Lett. B87,(1979),359.

[3] Dunkan A. and A.H.Mueller, Phys. Rev. D21,(1980),1636.

[4] Efremov A.V. and A.V.Radyushkin, Phys.Lett. B94,(1980),245.

[5] Farrar G.and D.Jackson, Phys. Rev.Lett. 43,(1979),246.

[6] V.L.Chernyak and A.R.Zhitnitsky,Phys.Rep. 112,(1984),173-318.

[7] A.Zhitnitsky, Phys.Lett.B325(1994),449.

A.Zhitnitsky,Phys.Lett. B329(1994),493.

[8] R. D. Peccei and H. Quinn, Phys. Rev. Letters 38, (1977), 1440.

S. Weinberg, Phys. Rev. Letters 40,(1978), 223;

F. Wilczek, Phys. Rev. Letters 40, (1978), 279.

[9] A. Zhitnitsky, Yad.Fiz. 31, (1980), 497 (Sov.J.Nucl. Phys. 31, (1980), 260).

[10] M. Dine, W. Fischler and M.Srednicki, Phys. Letters B104, (1981), 199;

[11] R.Stroynowski and A.Zhitnitsky, SMU preprint SMU-HEP-94-12, August 1994. Submitted to Phys. Rev.D. 


\section{1) Budget Explanation}

\subsection{Introduction}

Since May, 1991, when DOE first began to support it, the theory group at SMU has continued to enjoy steady progress. With the recent additions of Hornbostel and Vega the group has now grown to a total of five tenured and tenure-track faculty members (Hornbostel, McCartor, Olness, Teplitz, and Vega). Although this is our first proposal to $\mathrm{DOE}$ as a group, our list of publications and invited talks indicates that we are already achieving a level of breadth and productivity comparable to well established groups. We are now at a point where support for two postdoctoral positions in our group would seem very reasonable in normal budget times while one postdoc would appear absolutely necessary and fully justified even in the current budget climate. Since 1991 we have been supporting one or two postdoctoral positions by combining funds from a variety of sources like special university startup funds (used for Robertson), the TNRLC and private funding. This last year we have even used part of our travel funds in order to help pay one of our postdocs. With the loss of SSC-related funding we will not be able to continue supporting postdoctoral positions; funding of postdoctoral positions by DOE will be crucial to the vitality of the group.

Our budget proposal also includes requests for summer salaries, travel, graduate student support, and a modest amount for visitors and computers. Below we itemize each request. We are painfully aware of the budget constraints at DOE and have limited ourselves to those requests that we feel are necessary to maintain the vitality and competitiveness of a young, emerging group.

\subsection{Personnel Support}

- Summer Salaries We request two months summer support for McCartor, Hornbostel, Olness, and Vega.

\section{- Postdoctoral Researchers}

We request funding for one postdoctoral position in the first year of the new grant period. The funding here would be used to support Riemersma for the first half of 1995 after which we would hire a new postdoc. We request the addition of a second postdoc in the middle of the second year (September) of the new grant period. We point out that in our short existence as a group we have had a fair amount of success at selecting talented people and hence being able to place them in new jobs. Our first two finite-term appointments secured good jobs after leaving SMU. The first, visiting assistant professor Gilles Couture, went on to a faculty position at the University of Quebec in Canada. Our second, postdoc Dave Robertson, went on to a postdoctoral position at Ohio state.

- Computer System Administrator

We request support for one quarter time for our computer system administrator, Rakesh Jain. The theory group now has two DEC Alpha workstations, two SPARCstation ELC's connected to a SPARCstation II, and various other personal computers which are maintained by Jain. In the past, Jain has been fully supported by the HEP experimental group. However, since we have been using Rakesh Jain for at least one quarter of his time it seems proper that our budget reflect that portion 
of his time. Needless to say his help is absolutely necessary.

- Graduate Student

We are requesting support for a very promising graduate student, Boris Chibisov, who is just completing his course work and has already started research activities under the direction of McCartor. Mr. Chibisov would be our first theory graduate student. We have another graduate student that has indicated interest in theory, $\mathrm{G}$. Bao, but he is still engaged in course work.

- Visitor

The group at SMU works in collaboration with many physicists around the world. While modern communications such as E-Mail, and discussions at meetings and during short visits suffice for most of the needs of these collaborations, there is occasional need for an intense collaboration of several weeks duration. We are therefore requesting funds for a visit of several weeks duration for a senior visitor. The list of collaborators who have expressed interest in a visit if funding were available includes: Antonio Bassetto (INFN); Bohdan Grzadkowski (Warsaw University); Yuji Nakawaki (Setsunan University).

\subsection{Travel}

We are requesting travel funds in the amount of $\$ 4 \mathrm{~K}$ per faculty, $\$ 2 \mathrm{~K}$ per postdoc, and $\$ 0.5 \mathrm{~K}$ per graduate student. This funding will allow us to continue participating at national and international conferences, to disseminate our results and to keep abreast of the latest developments in our field, and to visit our collaborators throughout the US.

\subsection{Computer Equipment and Supplies}

As most research nowadays the research conducted in our group is heavily computer dependent. At the present time the group has, as noted above, two DEC Alpha machines, two SPARCstation ELC's connected to a SPARCstation II, and several PC's. All of this equipment was acquired using University startup funds and private donations. So far, no DOE funds have been used to purchase computer equipment at SMU. We are requesting a modest amount of funds to purchase additional RAM for one of our Alphas, to purchase computer software, and to upgrade as the need arises. 


\section{2) Current and Pending Support:}

2.1 McCartor: Gary McCartor, along with other SMU faculty has a grant from ARPA to study treat y monitoring. The grant period is $9 / 22 / 93-1 / 31 / 95$ and is in the amount of $\$ 216,000$. Along with other SMU faculty he has a pending proposal at Phillips Lab. for the period 10/1/94-9/30/96 in the amount of $\$ 382,500$

2.2 Olness: As part of the CTEQ collaboration, Fredrick Olness has a pending proposal with Michigan State University. The proposed grant period is 6/1/94 to 5/31/97 with an SMU amount of $\$ 28,050$. This proposal is pending consideration with DOE and NSF.

2.3 Rosenbaum \& Teplitz: D. Rosenbaum and V. Teplitz have a pending proposal with NASA to study Astrophysics. The proposed grant period is 3 years, with an SMU amount of $\$ 100,000$. 


\section{1) SMU Theory Group Publications: 1991-1994}

\subsection{Refereed Publications}

[1] Precision $\Upsilon$ Spectroscopy from Nonrelativistic Lattice $Q C D$, C. T. H. Davies, K. Hornbostel, A. Langnau, G. P. Lepage, A. Lidsey, J. Shigemitsu, and J. Sloan, SCRI-94-39, OHSTPY-HEP-T-94-005, accepted for publication in Phys. Rev. D (1994).

[2] A New Determination of $M_{b}$ From Lattice $Q C D$, C. T. H. Davies, K. Hornbostel, A. Langnau, G. P. Lepage, A. Lidsey, C.J. Morningstar, J. Shigemitsu and J. Sloan, SCRI-94-57, OHSTPY-HEP-T-94-004, heplat/9404012, accepted for publication in Phys. Rev. Lett. (1994).

[3] A Precise Determination of $\alpha_{s}$ From Lattice QCD, C. T. H. Davies, K. Hornbostel, G. P. Lepage, A. Lidsey, J. Shigemitsu and J. Sloan, OHSTPY-HEP-T-94-013, FSU-SCRI-94-79, hep-ph/9408328, submitted to Phys. Lett. (1994).

[4] Schwinger Model in the Light-Cone Representation, Gary McCartor, to appear in Z. Phys. C (1994).

[5] Light-Cone Quantization of Gauge Fields, G. McCartor and D. G. Robertson, Z. Phys. C 62, 349 (1994).

[6] A Unified QCD Formulation of Charged Current and Neutral Current Heavy Quark Production in Deep Inelastic Scattering, M.A.G. Aivazis, F. Olness, and Wu-Ki Tung, Physical Review D50, 3085 (1994).

[7] A Unified QCD Formulation of Charged Current and Neutral Current Heavy Quark Production in Deep Inelastic Scattering II: Next to Leading Order Calculation, M.A.G. Aivazis, J. Collins, F. Olness, and Wu-Ki Tung, Physical Review D50, 3102 (1994).

[8] Leptoproduction of Heavy Quarks in the Fixed and Variable Flavor Schemes, F. Olness and S: Riemersma, SMU preprint SMU-HEP/94-21, August (1994). Submitted to Phys. Rev. D.

[9] Coupled Channel Vector Boson Scattering In The N/d Method, Duane A. Dicus and Vigdor L. Teplitz, Phys. Rev. D49, 5735 (1994).

[10] Improved Bounds on Non-Luminous Matter in Solar Orbit, J. D. Anderson, E. L. Lau, A. H. Taylor, D. A. Dicus, D. Rosenbaum, and Vigdor L. Teplitz. Submitted to Astrophysical Journal (1994).

[11] LHC Detection of Neutral MSSM Higgs Bosons via $g g \rightarrow h b \bar{b} \rightarrow b \bar{b} b \bar{b}$, J. Dai, J.F. Gunion, R. Vega, SMU preprint HEP-94-4, Mar 1994. Submitted for publication in Phys. Rev. D.

[12] Standard Model Decays of Tau into Three Charged Leptons, D.A. Dicus, R. Vega, SMU preprint HEP-94-2, Feb. 1994.

To appear in Phys. Lett. B.

[13] Constraints on CP Violation in the Higgs Sector From the $\rho$ Parameter, A. Pomarol, R. Vega, Nucl. Phys. B413 3 (1994). 
[14] Chiral and Heavy Quark Symmetry Violation in $b$ Decays, C.Glenn Boyd, B. Grinstein, SMU preprint HEP-94-3, Feb. 1994. Submitted for publication.

[15] Form-Factors in the Heavy Quark and Chiral Limit: Pole Dominance in $\bar{b} \rightarrow \pi e \bar{\nu}_{e}$ B. Grinstein, P.F. Mende, SSCL-PREPRINT-549, Jan. 1994.

Submitted for publication.

[16] Complete Next to Leading Order QCD Corrections to the Photon Structure Functions $F_{2}^{\gamma}\left(x, Q^{2}\right)$ and $F_{L}^{\gamma}\left(x, Q^{2}\right)$,

E. Laenen, S. Riemersma, J. Smith, and W.L. van Neerven, Phys. Rev. D49, 5753 (1994).

[17] Fits to the $O\left(\alpha_{s}\right)$ Corrected Coefficient Functions of Heavy-Flavor Structure Functions, S. Riemersma, J. Smith, and W.L. van Neerven, in preparation.

[18] What do Heavy-Light (Q Anti-Q) Quark Systems Tell Us About QCD Vacuum Properties?,

A. Zhitnitsky, Phys. Lett. B325 449 (1994).

[19] The Nonperturbative Wave Functions, Transverse Momentum Distribution and QCD Vacuum Structure,

A. Zhitnitsky, Phys. Lett. B329 493 (1994).

[20] Axion Search with Optical Technique

R. Stroynowski and A. Zhitnitsky, SMU preprint SMU-HEP-94-12, August 1994. Submitted to Phys. Rev. D.

[21] The Pion Form-Factor: Where Does it Come From?,

A.R. Zhitnitsky, SMU-HEP-94-01, Jan 1994. Submitted for publication.

[22] The Challenge of Light-Cone Quantization of Gauge Field Theory, G. McCartor, S. Brodsky, H.-C. Pauli and S. Pinsky, Particle World 3, 109 (1993).

[23] Handbook of Perturbative QCD: Version 1.0, CTEQ Collaboration (Raymond Brock, et al.), Fermilab-Pub-93-094, April (1993). Accepted for publication in Reviews of Modern Physics.

[24] Guaranteed Detection of a MSSM Higgs Boson at Hadron Supercolliders, J. Dai, J.F. Gunion, R. Vega, Phys. Lett. B315 355 (1993).

[25] Using $b$ Tagging to Detect $t \bar{t}$-Higgs-Boson Production with $H \rightarrow b \bar{b}$, J. Dai, J.F. Gunion, R. Vega, Phys. Rev. Lett. 712699 (1993).

[26] Complete $O\left(\alpha_{s}\right)$ Corrections to Heavy-Flavour Structure Functions in Electroproduction,

E. Laenen, S. Riemersma, J. Smith, W.L. van Neerven, Nucl. Phys. B392, 162 (1993).

[27] $O\left(\alpha_{s}\right)$ Corrections to Heavy-Flavour Inclusive Distributions in Electroproduction, E. Laenen, S. Riemersma, J. Smith, W.L. van Neerven, Nucl. Phys. B392, 229 (1993).

[28] On Spontaneous Symmetry Breaking In Discretized Light Cone Field Theory David G. Robertson, Phys. Rev. D47, 2549 (1993). 
[29] Improved Nonrelativistic QCD for Heavy Quark Physics, G. P. Lepage, L. Magnea, C. Nakhleh, U. Magnea and K. Hornbostel, Phys. Rev. D46, 4052 (1992).

[30] Nontrivial Vacua From Equal Time to the Light Cone, K. Hornbostel, Phys. Rev. D45, 3781 (1992).

[31] Bosonic Zero Modes in Discretized Light-Cone Field Theory, G. McCartor and D. Robertson, Z. Phys. C 53, 679 (1992).

[32] An Equal-Time Quantized Field Theory on the Light Cone, G. McCartor and D. Robertson, Z. Phys. C 53, 661 (1992).

[33] A Comparison of Phase Screen and Finite Difference Methods for Elastic Waves in Random Media,

G. McCartor, M. Fisk and T. Charrette, J. Geophys. Res. 97, 12409 (1992).

[34] Fluctuation Analysis of Elastic Waves in Random Media Via Phase Screen Simulations,

G. McCartor and M. Fisk, J. Geophys. Res. 98, 685 (1992).

[35] Semi-Inclusive Deeply Inelastic Scattering at Electron-Proton Colliders, R. Meng, F. Olness, and D. Soper. Nuclear Physics B371, 79 (1992).

[36] The Axial Vector Coupling and Magnetic Moment of the Quark, D.A. Dicus, D. Minic, U. van Kolck, R. Vega, Phys. Lett. B284 384 (1992).

[37] Isolating Purely Leptonic Signals for Strong $W$ Scattering Using Antitagging Jet Tagging, and Lepton Isolation,

D.A. Dicus, J.F. Gunion, L.H. Orr, R. Vega, Nucl. Phys. B377 31 (1992).

[38] Probing The WWZ Vertex At A $\sqrt{s}=500 \mathrm{GeV} e^{+} e^{-}$Collider, Gilles Couture, Stephen Godfrey, and Randy Lewis, Phys. Rev. D45, 777 (1992).

[39] Difficulties in Determining the Gluon Distribution from Electroproduction of Charmed Quarks,

S. Riemersma, J. Smith, and W.L. van Neerven, Phys. Lett. B282, 171 (1992).

[40] On the Heavy-Quark Content of the Nucleon,

E. Laenen, S. Riemersma, J. Smith, W.L. van Neerven, Phys. Lett. B291, 325 (1992).

[41] Review of Attenuation in Salt at Moderate Strains,

G. McCartor and W. R. Wortman, Geophys. Mon. 65, 99 (1991).

[42] Light-Cone Gauge Schwinger Model, Gary McCartor, Z. Phys. C 52, 611 (1991).

[43] A Phase Screen Method for Vector Elastic Waves, G. McCartor and M. Fisk, J. Geophys. Res. 96 (1991).

[44] Left-Right Symmetric Electroweak Models with Triplet Higgs, N.G. Deshpande, J.F. Gunion, B. Kayser, and F. Olness, Physical Review 44, 837 (1991).

[45] Isolating the Scattering of Longitudinal $W^{+}$'s at the SSC Using Like Sign Dileptons, D.A. Dicus, J.F. Gunion, R. Vega, Phys. Lett. B258 475 (1991). 
[46] Naturalness Problems for $\rho=1$ and Other Large One Loop Effects for a Standard Model Higgs Sector Containing Triplet Fields, J.F. Gunion, R. Vega, J. Wudka, Phys. Rev. D43 2322 (1991).

[47] On The Magnetic And Electric Dipole Moment Parameters Of The Tau Lepton, Gilles Couture, Phys. Lett. B272, 404 (1991).

\subsection{Other Publications}

[1] Leptoproduction of Heavy Quarks in the Fixed and Variable Flavor Schemes, F. Olness and S. Riemersma. Presentation by F. Olness at Particles \& Fields '94: Meeting of the Division of Particles \& Fields of the APS, Albuquerque, NM, 2-6 August 1994.

SMU preprint SMU-HEP/94-22, August (1994).

[2] Semi-Inclusive Deeply Inelastic Scattering at Electron-Proton Colliders: From Small to Large $P_{T}$,

R. Meng, F. Olness, and D. Soper, SMU preprint SMU-HEP/94-13, in preparation.

[3] Heavy Quark Production in Deep Inelastic Scattering at HERA,

P. Agrawal, F. Olness, S. Riemersma, and Wu-Ki Tung, SMU preprint in preparation.

[4] Light Cone Quantized QCD,

K. Hornbostel, Invited talk at Workshop on QCD Vacuum Structure, Paris, France, Jun 1-5, 1992 (World Scientific, Singapore, 1993).

[5] A Unified QCD Formulation of Charged Current and Neutral Current Heavy Quark Production in Deep Inelastic Scattering, M.A.G. Aivazis, F. Olness, and Wu-Ki Tung, The Fermilab Meeting DPF'92, ed. C. Albright et al., p.981 (1993).

[6] GEM Technical Design Report, W.C. Lefmann, et al., GEM-TN-93-262, Apr. (1993).

[7] B-Quark Production at Hadron Colliders,

S. Riemersma and R. Meng, SMU HEP 93-08, ANL-HEP-CP-93-66, Proceedings of Workshop on B-Physics at Hadron Accelerators, Snowmass '93.

[8] Light Cone QCD in a Temporal Gauge,

K. Hornbostel, Proceedings of the SMU Workshop on Light Cone Quantization, (unpublished) Southern Methodist University, Dallas, Texas (1992).

[9] Structure Functions and Parton Distribution Functions,

F. Olness and W. Tung, Proceedings of the 1990 DPF Summer Study on High Energy Physics: Research Directions for the Decade, Snowmass, CO, p. 148, (1992).

[10] ep Collider Experiments and Physics,

D. Atwood, U. Baur, J. Blümlein, G. Couture, M. A. Doncheski, R. Fletcher, D. Goddard, S. Godfrey, J. L. Hewett, D. P. Johnson, S. Keller, B. A. Kniehl, G. Levman, R. Lewis, L. Marleau, S. Mani, R. Meng, F. Olness, R. Orr, D. Reeder, T. G. Rizzo, G. Schuler, W. H. Smith, H. Spiesberger, K. Sugano, and R. Talaga. Proceedings of the 1990 DPF Summer Study on High Energy Physics: Research Directions for the Decade, Snowmass, CO, p. 531, (1992).

[11] Kinematic Coverage of Structure Function Measurements, G.A. Schuler, J. Blümlein, F. Olness, and Wu-Ki Tung. Proceedings of the 1990 DPF Summer Study on High Energy Physics: Research Directions for the Decade, Snowmass, CO, p. 152, (1992). 
[12] Higgs Bosons in the Minimal Supersymmetric Extension of the Standard Model, A. Brignole, J. Ellis, J.F. Gunion, M. Guzzo, F. Olness, G. Ridolf, L. Roszkowski, and F. Zwirner. Proceedings of the Workshop: $e^{+} e^{-}$Linear Colliders at $500 \mathrm{GeV}$ : the Physics Potential, Ed. P.M. Zerwas, p.613, (1992).

[13] Next-to-Leading Order QCD Formulation of Deep Inelastic Scattering, M.A.G. Aivazis, F. Olness, and Wu-Ki Tung.

Presentation by F. Olness at Particles \& Fields '91: Meeting of the Division of Particles \& Fields of the APS, Vancouver, BC, Canada. 18-22 August, 1991.

The Vancouver Meeting-Particles and Fields '91, (ed. D. Axen, et. al), p. 663 (1992).

[14] Charm Production at HERA,

S. Riemersma, Proceedings of DPF '92, The Fermilab Meeting, p. 984.

[15] GEM Letter of Intent, The GEM Collaboration, SSC Laboratory publication SSCL-SR-1184, Nov. 1991. 


\section{1) Seminar \& Conference Presentations by SMU Theory Group}

\subsection{Presentations: Kent Hornbostel}

[1] Massachusetts Institute of Technology, invited seminar (1993).

[2] Baylor University, invited seminar (1993).

[3] Ohio State University, invited seminar (1992).

[4] Southern Methodist University, invited seminar (1991, 1992).

[5] Cornell University, invited seminar (1992).

[6] SMU Workshop on Light Cone Quantization, Southern Methodist University, Dallas, Texas, invited talk (1992).

[7] Workshop on QCD Vacuum Structure, American University of Paris, Paris, France, invited talk (1992).

[8] Workshop on Light-Cone Quantization, MPI Heidelberg, Germany, invited talk (1991).

[9] Aspen Light-Cone Workshop, Aspen Center for Physics, Aspen, Colorado, invited talk (1991).

[10] Duke University, invited seminar (1991).

\subsection{Presentations \& Committees: Gary McCartor}

COMMitTEEs::

We hosted the second international meeting on light-cone quantization at SMU in 1992. Gary McCartor is a member of the international committee on light-cone meetings and has been on the advisory committee for the meetings at Telluride(1992); Zurich(1993); Gran Sasso(1993); Seattle(1994); and Zakopane(1994).

\section{RESEARCH VISITS:}

Gary McCartor made a month-long visit to Padua in Spring,1994, and has scheduled month-long visits to Ohio State and SLAC in Fall 1994.

\section{SEMINARS::}

[1] Ohio State(1991)

[2] SSC Laboratory(1991)

[3] Washington University(1991)

[4] Heidelberg(1992)

[5] SLAC(1992)

[6] Zurich(1992)

[7] Regansburg(1992)

[8] Argonne(1992)

[9] Texas A\&M(1993)

[10] SLAC(1993)

[11] University of North Texas(1993) 
[12] Nagoya(1993)

[13] Heidelberg(1993)

[14] Padua(1994)

[15] Trento(1994)

CONFERENCE TALKS::

[16] Heidelberg 6/91

[17] Aspen 9/91

[18] Telluride $9 / 92$

[19] Zurich 6/93

[20] Gran Sasso 9/93

[21] Zakopane $9 / 94$

\subsection{Presentations: Fredrick Olness}

Conference \& Summer School Presentations: 1991-94:

[1] SSC Physics Symposium, The QCD Parton Model and NLO Corrections, Madison, WI. 25-27 February, 1991.

[2] DPF 1991: Next-to-Leading Order QCD Formulation of Deep Inelastic Scattering, Vancouver, BC, Canada. 18-22 August, 1991.

[3] SSC Physics Symposium, Precision QCD for the SSC, Madison, WI. 13-15 April, 1992.

[4] DPF 1992: A Unified QCD Analysis of Chärged Current and Neutral Current Heavy Quark Production in Deep Inelastic Scattering , Fermilab, Batavia, IL. 10-14 November, 1992.

[5] CTEQ Summer School on QCD Analysis and Phenomenology: Deeply Inelastic Scattering, 25 July - 3 August 1993, Lake Monroe, IN

[6] Physics Doesn't Stop, Madison Physics Symposium, Madison, WI. 11-13 April, 1993.

[7] DPF 1994: Leptoproduction of Heavy Quarks in the Fixed and Variable Flavor Schemes, Albuquerque, NM, 2-6 August 1994.

Seminar and Colloquium Presentations: 1991-94:

[8] The University of Texas-Austin Theory Seminar: Tests of $Q C D$ at Electron Proton Colliders: Semi-Inclusive Deep Inelastic Scattering, 25 April, 1994.

[9] Duke University Seminar: A Unified QCD Formulation of Heavy Quark Production, 8 November, 1993.

[10] Columbia University Seminar: A Unified QCD Formulation of Heavy Quark Production, 12 March, 1993.

[11] Brookhaven National Laboratory Theory Seminar: A Unified QCD Formulation of Heavy Quark Production, 17 March, 1993.

[12] The University of Texas-Austin Theory Seminar: A Unified QCD Formulation of Heavy Quark Production, 25 January, 1993. 
[13] GEM Collaboration Physics Seminar: Parton distributions and their effects on Monte Carlo results - problems and fallacies, SSC Laboratory, Dallas, TX, 1 April, 1992.

[14] Michigan State University Seminar How strange is the proton? (Charm production in Deeply-Inelastic Scattering), March 12, 1992.

[15] SSC Symposium at SMU: What we know, and what we need to know about the Standard Model? , November 12, 1991.

[16] Superconducting SuperCollider Laboratory Colloquium: What is Really Inside a Proton?, October 31, 1991.

[17] Oregon State University Seminar: What is really inside a Proton? The Quantum Chromodynamic Parton Model and Higher Order Corrections, April 18, 1991.

[18] Stanford Linear Accelerator Center (SLAC) Theory Seminar: The Quantum Chromodynamic Parton Model and Higher Order Corrections, March 20, 1991.

[19] Southern Methodist University Colloquium: What is really inside a Proton? The Quantum Chromodynamic Parton Model and Higher Order Corrections, March 5, 1991.

[20] University of Texas-Austin Seminar: The Quantum Chromodynamic Parton Model and Higher Order Corrections, March 4, 1991.

[21] Florida State University Seminar: Tests of QCD at Electron Proton Colliders: SemiInclusive Deep Inelastic Scattering, January 27, 1991.

[22] Lawrence Berkley 4-D Lunch Seminar: The Quantum Chromodynamic Parton Model and Higher Order Corrections, January 17, 1991.

[23] Argonne National Laboratory Seminar: Tests of QCD at Electron Proton Colliders: Semi-Inclusive Deep Inelastic Scattering, January 7, 1991.

\subsection{Presentations: Roberto Vega}

Seminar \& Conference Presentations: 1991-94:

[1] National Meeting of the Mexican Society of High Energy Physics, Hunting for Higgs Particles at Future Colliders, Mexico DF. July, 1994.

[2] 17th International Warsaw Meeting on Elementary Particle Physics, Closing the Window on MSSM Higgs Searches, Kazimierz, Poland. May, 1994.

[3] Florida State University Seminar: The Search for Susy Higgs. November, 1993.

[4] SLAC Seminar: The Search for an Intermediate Mass Higgs. July, 1993.

[5] New York University Seminar: Searching for Heavy Higgs at the SSC. July, 1992.

[6] New York University Seminar: The Axial Vector Coupling and Magnetic Moment of the Quark. July, 1992.

[7] McGill University Seminar: Higgs Searches at the Superconducting Supercollider. July, 1992.

[8] SSC Physics Symposium, A New Helicity Method for Massive Fermions, Madison, WI. April, 1992. 
[9] National Academy of Sciences Ford Fellowship Conference, The Superconducting Supercollider: the Largest Scientific Instrument Ever Built, Washington, D.C. October, 1992. 


\section{2) SMU High Energy Seminar Schedule: 1993}

\subsection{Spring 1993}

January 5, 1993

January 12, 1993

January 26, 1993

February 2, 1993

February 16, 1993

February 17, 1993

February 19,1993

February 26,1993

March 2, 1993

March 3, 1993

March 9, 1993

March 12, 1993

March 23, 1993

March 24, 1993

April 20, 1993

May 3, 1993

May 12, 1993

May 13, 1993

\subsection{Fall 1993}

17-Sep-93

8-Oct-93

$11-$ Oct-93

18-Oct-93

22-Oct-93

25-Oct-93

29-Oct-93

1-Nov-93

8-Nov-93

10-Nov-93

12-Nov-93

15-Nov-93

19-Nov-93

22-Nov-93

3-Dec-93

6-Dec-93

10-Dec-93

13-Dec-93
Jay Dominick

Beale Ong

C. Burgess

Andrew Bazarko

G. Chew

John Hobbs

Giorgio Gratta

Stan Brodsky

Pete Lepage

Pete Lepage

Adam Falk

Donald Clayton

Misha Shifman

Alex Pomarol

J. Mitchell

V. Kadyshevsky

R. Arnowitt

P. Ramond
Purdue

UCSD

McGill

Columbia

LBL

CERN

Caltech

SLAC

Cornell

Cornell

SLAC

Clemson

Minnesota

Santa Cruz

McGill/DESY

JINR Dubna

Texas A\&M

Florida
Mark Lambrecht

David Bowser-Chao

Peter van Baal

Bryan W.Lynn

Yue Shen

Vic Teplitz

Eugen Levin

Martin Shub

Alex de Lozanne

J.F.Gunion

Howie Baer

Elliot Cheu

Alfred Mueller

Kenneth R.

Walter Giele

Thomas Coan

Chung Kao

John Harton
OSU/SMU

Michigan State U.

Utrecht U.

Stanford U.

$\mathrm{BU}$ (SSC)

SMU

Fermilab

U. of Chicago

U. of Texas at Austin

UC Davis

FSU

U. of Chicago

Columbia

U. of Texas at Houston

Fermilab

Los Alamos (CERN)

FSU

U. of Wisconsin (CERN) 


\section{CURRICULUM VITAE}

September 9, 1994

\section{KENT HORNBOSTEL}

Department of Physics

Southern Methodist University

Dallas, TX 75275

2147681048 (office) 2147390355 (home)

$\begin{array}{ll}\text { Born: } & 9 \text { August } 1959 \text { Omaha, Nebraska } \\ \text { Citizenship: } & \text { United States }\end{array}$

EDUCATION

9/81-9/88 Stanford University and Stanford Linear Acclerator Center

Ph.D. (Physics)

Thesis advisor: Professor Stanley J. Brodsky

Thesis: The Application of Light-Cone Quantization to $Q C D$ in $(1+1)$ Dimensions

9/77-9/81 Duke University, B.S. (Physics)

Angier B. Duke scholar; Phi Beta Kappa;

Graduated summa cum laude

EMPLOYMENT

1/93 - Present Department of Physics, Southern Methodist University

Assistant Professor

10/91-12/92 Department of Physics, Ohio State University

University Postdoctoral Fellow

9/88-10/91 Newman Laboratory of Nuclear Studies, Cornell University

Postdoctoral Associate

9/81-6/82 Stanford University

Teaching Assistant (introductory physics)

Summer 1980 Princeton Plasma Physics Lab and

Grumman Aerospace Corporation

Summers 1977-79 Grumman Aerospace Corporation, Bethpage, NY 


\section{VISITOR}

8/86-9/86 Max-Planck Institute für Kernphysik

Heidelberg, Germany

8/85-1/86 Institute for Theoretical Physics

University of California, Santa Barbara

Workshop on Nuclear Chromodynamics

\section{PUBLICATIONS}

1. Baryon Masses in the Bound State Approach to Strangeness in the Skyrme Model, C. G. Callan, K. Hornbostel and I. Klebanov, Phys. Lett. 202B, 269, (1988).

2. Quantization on the Light Cone: Response to a Comment by C. R. Hagen, K. Hornbostel, S. J. Brodsky and H.-C. Pauli, Phys. Rev. D 37, 2363, (1988).

3. The Application of Light-Cone Quantization to QCD in (1+1) Dimensions, K. Hornbostel, Ph. D. Thesis, SLAC-0333, 1988.

4. Light Cone Quantized QCD in (1+1) Dimensions, K. Hornbostel, S. J. Brodsky and H.-C. Pauli, Invited talk presented to the Workshop on Relativistic Many Body Physics, Columbus, Ohio, Jun 6-9, 1988, (World Scientific, Singapore, 1988).

5. Light Cone Quantized QCD in (1+1) Dimensions,

K. Hornbostel, S. J. Brodsky and H.-C. Pauli, Phys. Rev. D 41, 3814, (1990).

6. Building Hadrons on the Light Cone, K. Hornbostel, Invited talk presented to the Boulder Workshop "From Fundamental Fields to Nuclear Phenomena," Boulder, Colorado, Sept 6-9, 1990, (World Scientific, Singapore, 1990).

7. Nontrivial Vacua From Equal Time to the Light Cone, K. Hornbostel, Phys. Rev. D 45, 3781 (1992).

8. Improved Nonrelativistic QCD for Heavy Quark Physics, G. P. Lepage, L. Magnea, C. Nakhleh, U. Magnea and K. Hornbostel, Phys. Rev. D 46, 4052 (1992).

9. Light Cone Quantized QCD,

K. Hornbostel, Invited talk at Workshop on QCD Vacuum Structure, Paris, France, Jun 1-5, 1992, (World Scientific, Singapore, 1993).

10. Precision $\Upsilon$ Spectroscopy from Nonrelativistic Lattice $Q C D$,

C. T. H. Davies, K. Hornbostel, A. Langnau, G. P. Lepage, A. Lidsey, J. Shigemitsu, and J. Sloan, SCRI-94-39, OHSTPY-HEP-T-94-005, hep-lat/9406017, to appear to Phys. Rev. $\mathrm{D}$ (1994).

11. New Determination of $M_{6}$ From Lattice $Q C D$, C. T. H. Davies, K. Hornbostel, A. Langnau, G. P. Lepage, A. Lidsey, C.J. Morningstar, J. Shigemitsu, J. Sloan, SCRI-94-57, OHSTPY-HEP-T-94-004, hep-lat/9404012, to appear to Phys. Rev. Lett. (1994). 
12. A Precise Determination of $\alpha_{s}$ From Lattice $Q C D$,

C. T. H. Davies, K. Hornbostel, G. P. Lepage, A. Lidsey, J. Shigemitsu, J. Sloan, OHSTPY-

HEP-T-94-013, FSU-SCRI-94-79, hep-ph/9408328, submitted to Phys. Lett. (1994).

\section{WORK IN PROGRESS}

1. Boundary Degrees of Freedom and Chiral Symmetry in Light-Cone Quantization, K. Hornbostel, D. Robertson and G. McCartor.

2. Temporal Gauge $Q C D$ on a Light-Cone Lattice, K. Hornbostel.

3. Contribution of Higher-Fock Wavefunctions to Deep Inelastic Structure Functions, S. J. Brodsky and K. Hornbostel.

\section{INVITED CONFERENCE TALKS}

1. Workshop on Relativistic Nuclear Many-Body Physics

Ohio State Univesity, Columbus, Ohio, 1988.

2. From Fundamental Fields to Nuclear Phenomena Boulder, Colorado, 1990.

3. Workshop on Light-Cone Quantization MPI Heidelberg, Germany, 1991.

4. Aspen Light-Cone Workshop Aspen Center for Physics, Aspen, Colorado, 1991.

5. SMU Workshop on Light Cone Quantization

Southern Methodist University, Dallas, Texas, 1992.

6. Workshop on QCD Vacuum Structure

American University of Paris, Paris, France, 1992.

\section{INVITED SEMINAR TALKS}

1. Stanford Linear Accelerator Center (1987)

2. Fermi National Laboratory (1989)

3. Ohio State University $(1990,1992)$

4. Duke University (1991)

5. Southern Methodist University $(1991,1992)$

6. Cornell University $(1988,1992)$

7. Massachusetts Institute of Technology (1993)

8. Baylor University (1993) 


\section{CURRICULUM VITAE}

\section{Gary Don McCartor}

Department of Physics

Southern Methodist University

Dallas, TX 75275

(214) 768-3693

mccartor@smuphy.physics.smu.edu

\section{PERSONAL:}

Born: 28 April 1943, Amarillo, Texas

Citizenship: USA

EDUCATION:

A.B. Occidental College, 1965 (Physics-Mathematics)

Ph.D. Texas A\&M University, 1969 (Physics)

Dissertation Title: Topics in Scattering Theory

Thesis advisor: John Nuttall

\section{EMPLOYMENT:}

$\begin{array}{ll}1990-\text { Present } & \text { Professor of Physics, Southern Methodist University } \\ 1971-1989 & \text { Member technical staff, Mission Research Corporation } \\ & \text { Last position: Division Leader } \\ 1976-1977 & \text { Visiting Professor, Saint Louis University } \\ 1969-1971 & \begin{array}{l}\text { NSF Post-doctoral Fellow, Manned Spacecraft Center, } \\ \text { Houston, Texas }\end{array}\end{array}$

SHORTER VISITS TO:

Summer 1985

Los Alamos National Laboratory

Spring 1988

Max Planck Institute (Heidelberg)

Fall 1989

Max Planck Institute (Heidelberg)

Spring 1994

University of Padua

CURRENT COMMITTEES:

International Committee on Light-Cone Meetings

Scientific Advisory Committee, Seattle Meeting (1994)

Scientific Advisory Committee, Poland Meeting (1994) 


\section{PUBLICATIONS:}

"Schwinger Model in the Light-Cone Representation"

Gary McCartor

SMU preprint SMUHEP/93-19

Z. Phys. $\mathbf{C}$ in press

"Light-Cone Quantization of Gauge Fields"

with D.G. Robertson

Z. Phys. C 62 (1994) 349

"The Challenge of Light-Cone Quantization of Gauge Field Theory" with S. Brodsky,H-C Pauli and S. Pinsky

Particle World 3 (1993) 109

"Bosonic Zero Modes in Discretized Light-Cone Field Theory" with D. Robertson

Z. Phys. C 53 (1992) 679

"An Equal-Time Quantized Field Theory on the Light Cone" with D. Robertson

Z. Phys. C 53 (1992) 661

"Review of Attenuation in Salt at Moderate Strains"

with W. R. Wortman

Geophys. Mon. 65 (1991) 99

"Light-Cone Gauge Schwinger Model"

Gary McCartor

Z. Phys. C 52 (1991) 611

"Fluctuation Analysis of Elastic Waves in Random Media Via

Phase Screen Simulations"

with M. Fisk

J. Geophys. Res. 98 (1992) 685

"A Comparison of Phase Screen and Finite Difference Methods for Elastic Waves in Random Media"

with M. Fisk and T. Charrette

J. Geophys. Res. 97 (1992) 12409

"A Phase Screen Method for Vector Elastic Waves"

with M. Fisk

J. Geophys. Res. 96 (1991)

"Analysis of Nuclear Explosion Salmon Free-Field Ground Motion Data for Nonlinear Attenuation"

with W. R. Wortman

J. Geophys. Res. 95 (1990) 
"The Initial Value Problem for Acoustic Gravity Waves"

Gary McCartor

Quart. App. Math. XLVII, No. 11 (1989)

"Testing for the Maximum Mean in a Mixture of Normals"

with others

Comm. Stat. 18, No. 11 (1989)

"Light-Cone Quantization for Massless Fields"

Gary McCartor

Z. Phys. C41 (1988) 271

"Light-Cone Quantization for Massless Fields"

Gary McCartor, in Nuclear and Particle Physics on the Light-Cone,

Mikkel Johnson and L.S. Kisslinger, eds. (World Scientific, 1988)

"Perturbation Calculations for Infrared-Divergent Problems"

Gary McCartor

Phys. Rev. D32, No. 8 (1985)

"Nonrelativistic Quark Models"

Gary McCartor

Phys. Rev. D15, No. 6 (1978)

"Error Bounds for the Complex Coordinate Method"

Gary McCartor

J. Math. Phys. 19 (1978)

"Vortex States in Non-Rotating Liquid $\mathrm{He}_{4}$ "

Gary McCartor

J. Low Temp. Phys. 19 (1978)

"Contour Distortions and the Bethe-Salpeter Equation"

with J. Nuttall

Phys. Rev. A4, No. 2 (1971)

"Calculation of Ionization Amplitudes by the Complex-Energy Method" with J. Nuttall

Phys. Rev. A4, No. 1 (1971)

"S-Wave Elastic Position-Hydrogen Scattering in the Ionization Region" with others

Phys. Rev. A4, No. 1 (1971)

\section{REPORTS:}

A list of over sixty reports is omitted; it is available upon request. 


\section{FREDRICK IVER OLNESS}

\section{Curriculum Vitae}

Southern Methodist University

Work Phone: (214) 768-2500

Dallas, Texas 75275

olness@smuphy.physics.smu.edu

Work Fax: (214) 768-4095

Home Phone: (214) 739-2800

\section{Education:}

- Ph.D. in Theoretical Particle Physics, Minor in Mathematics. University of Wisconsin-Madison, August 1985.

- M.S. in Physics. University of Wisconsin-Madison, August 1982.

- B.S. in Physics and Mathematics. Duke University, May 1980. Honors Research Thesis, Graduated with Distinction in Physics; Magna Cum Laude.

\section{Academic Positions:}

- Southern Methodist University, Department of Physics, Theoretical Particle Physics Group. Assistant Professor. August 1991 to Present.

- Superconducting SuperCollider Laboratory, SSC Fellow 1993-94. Physics Research, Guest Scientist. August 1991 to Present.

- University of Oregon, Institute of Theoretical Science, Theoretical Particle Physics Group. Postdoctoral Research Fellow. September 1988 to August 1991.

- Universität Dortmund, Institut für Theoretische Physik. Visiting faculty member, guest of Prof. E. A. Paschos. April-May 1989.

- Illinois Institute of Technology, Theoretical Particle Physics Group. Postdoctoral Research Fellow. September 1985 to August 1988.

- University of Wisconsin-Madison, Theoretical Particle Physics Group. Research Assistant. May 1982 to August 1985.

- University of Wisconsin-Madison, Experimental High Energy Physics Group. Research Assistant. Assisted in the preparation of experiment E609 at Fermilab. Summer 1981.

- Los Alamos Scientific Laboratory. Research Assistant. Developed computer codes to simulate multiphase fluid flow on CDC 7600 and Cray 1 systems. Summers 1979-80.

- Duke University, Triangle Universities Nuclear Laboratory. Research Assistant. Performed and analyzed a ${ }^{13} C(p, \gamma)^{14} N$ experiment as a portion of Honors Research Thesis. September 1979 to May 1980. 


\section{Professional Activities:}

- Lecturer at CTEQ Summer School on QCD Analysis and Phenomenology, 25 July - 3 August 1993, Lake Monroe, IN

- Leader, Structure Functions sub-group with J. Owens \& W. Tung. 1993 SSC Madison Workshop, 29-31 March 1993.

- Member of the Coordinated Theoretical/Experimental Project on Quantitative QCD (CTEQ) collaboration

- Member of the GEM (Gammas, Electrons, Muons) Collaboration.

- Referee for The Physical Review.

- Grant proposal reviewer for the National Science Foundation, and Department of Energy.

- Memberships: Sigma Xi Scientific Research Society; American Physical Society; American Institute of Physics.

\section{Awards and Scholarships:}

- SSC Fellowship, 1993-94.

- Scholarship to attend Theoretical Advanced Study Institute in Elementary Particle Physics, Yale University, June 9-July 5, 1985.

- Graduate School Dean's Fellowship, University of Wisconsin-Madison, 1983-84. One of ten selected in a university-wide competition.

- Associated Universities Incorporated Scholarship, awarded through Brookhaven National Laboratory, 1976-1980. One of ten selected in a merit competition.

\section{Teaching Experience:}

- Southern Methodist University: General Physics 1303 \& 1304: Newtonian Mechanics. Solid State Physics 5337. Graduate Classical Mechanics 6321. Teaching evaluations by students are consistently 9 on a scale of 10 .

- University of Oregon: General Physics: Newtonian Mechanics. Fall Term 1989. Enrollment of 208 students; 4 lectures and 2 help sessions per week; supervised 3 graduate teaching assistants; wrote exams and quizzes. Based on formal university survey, received outstanding recommendations from the students: rated 4.22 of $5.00 ; 12$ year department average, 3.68 of 5.00 .

- University of Wisconsin-Madison: Teaching Assistant for 4 semesters in General Physics. Taught 4 one hour recitations and 2 three hour labs per week. Approximately 20 students per class. $9 / 80$ to $5 / 82$. 


\section{Recent Publications:}

Refereed Publications: 1991-94

- Left-Right Symmetric Electroweak Models with Triplet Higgs, N.G. Deshpande, J.F. Gunion, B. Kayser, and F. Olness.

Physical Review 44, 837 (1991).

- Semi-Inclusive Deeply Inelastic Scattering at Electron-Proton Colliders, R. Meng, F. Olness, and D. Soper.

Nuclear Physics B371, 79 (1992).

- Handbook of Perturbative QCD: Version 1.0, CTEQ Collaboration (Raymond Brock, et al.), Fermilab-Pub-93-094, April (1993).

Accepted for publication in Reviews of Modern Physics.

- A Unified QCD Formulation of Charged Current and Neutral Current Heavy Quark Production in Deep Inelastic Scattering, M.A.G. Aivazis, F. Olness, and Wu-Ki Tung.

Physical Review D50, 3085 (1994).

- A Unified QCD Formulation of Charged Current and Neutral Current Heavy Quark Production in Deep Inelastic Scattering II: Next to Leading Order Calculation, M.A.G. Aivazis, J. Collins, F. Olness, and Wu-Ki Tung.

Physical Review D50, 3102 (1994).

- Leptoproduction of Heavy Quarks in the Fixed and Variable Flavor Schemes, F. OIness and S. Riemersma.

SMU preprint SMU-HEP/94-21, August (1994). Submitted to Phys. Rev. D.

Other Publications: 1991-94

- Next-to-Leading Order QCD Formulation of Deep Inelastic Scattering, M.A.G. Aivazis, F. Olness, and Wu-Ki Tung.

Presentation by F. Olness at Particles \& Fields '91: Meeting of the Division of Particles \& Fields of the APS, Vancouver, BC, Canada. 18-22 August, 1991.

The Vancouver Meeting-Particles and Fields '91, (ed. D. Axen, et. al), p. 663 (1992).

- GEM Letter of Intent. The GEM Collaboration. SSC Laboratory publication SSCL-SR-1184, Nov. 1991.

- Structure Functions and Parton Distribution Functions, F. Olness and W. Tung. Proceedings of the 1990 DPF Summer Study on High Energy Physics: Research Directions for the Decade, Snowmass, CO, p. 148, (1992). 
- ep Collider Experiments and Physics, D. Atwood, U. Baur, J. Blümlein, G. Couture, M. A. Doncheski, R. Fletcher, D. Goddard, S. Godfrey, J. L. Hewett, D. P. Johnson, S. Keller, B. A. Kniehl, G. Levman, R. Lewis, L. Marleau, S. Mani, R. Meng, F. Olness, R. Orr, D. Reeder, T. G. Rizzo, G. Schuler, W. H. Smith, H. Spiesberger, K. Sugano, and R. Talaga.

Proceedings of the 1990 DPF Summer Study on High Energy Physics: Research Directions for the Decade, Snowmass, CO, p. 531, (1992).

- Kinematic Coverage of Structure Function Measurements, G.A. Schuler, J. Blümlein, F. Olness, and Wu-Ki Tung. Proceedings of the 1990 DPF Summer Study on High Energy Physics: Research Directions for the Decade, Snowmass, CO, p. 152, (1992).

- Higgs bosons in the minimal supersymmetric extension of the Standard Model. A. Brignole, J. Ellis, J.F. Gunion, M. Guzzo, F. Olness, G. Ridolfi, L. Roszkowski, and F. Zwirner. Proceedings of the Workshop: $e^{+} e^{-}$Linear Colliders at $500 \mathrm{GeV}$ : the Physics Potential, Ed. P.M. Zerwas, p.613, (1992).

- A Unified QCD Formulation of Charged Current and Neutral Current Heavy Quark Production in Deep Inelastic Scattering, M.A.G. Aivazis, F. Olness, and Wu-Ki Tung.

The Fermilab Meeting DPF'92, ed. C. Albright et al., p.981 (1993).

- GEM Technical Design Report, W.C. Lefmann, et al., GEM-TN-93-262, Apr. (1993).

- Leptoproduction of Heavy Quarks in the Fixed and Variable Flavor Schemes, F. Olness and S. Riemersma.

Presentation by F. Olness at Particles \& Fields '94: Meeting of the Division of Particles \& Fields of the APS, Albuquerque, NM, 2-6 August 1994.

SMU preprint SMU-HEP/94-22, August (1994).

- Semi-Inclusive Deeply Inelastic Scattering at Electron-Proton Colliders: From Small to Large $P_{T}, \mathrm{R}$. Meng, F. Olness, and D. Soper. SMU preprint SMU-HEP/94-13, in preparation.

- Heavy Quark Production in Deep Inelastic Scattering at HERA, P. Agrawal, F. Olness, S. Riemersma, and Wu-Ki Tung.

SMU preprint in preparation. 


\title{
ROBERTO VEGA
}

(Sept. 1994)

\author{
Department of Physics \\ Southern Methodist University \\ Dallas, TX 75275 \\ (214) $768-2498$ \\ email: vega@smuphy.physics.smu.edu
}

EDUCATION Ph.D. (Theoretical Physics) University of Texas at Austin, 1988.

Dissertation Topic: WW Physics and the Higgs Boson at the SSC. (Thesis Advisor: Prof. Duane A. Dicus)

M.S. (Theoretical Physics) Georgia Institute of Technology, 1982.

B.S. (Physics) University of Puerto Rico at Mayaguez, 1978.

ACADEMIC Assistant Professor- Southern Methodist University (1993-Present)

POSITIONS Research Associate- Stanford Linear Accelerator Center. (1991-1993)

Visiting Research Physicist- Stanford Linear Accelerator Center. (1990-1991)

Postdoctoral Research Associate- University of California at Davis. (1988-1990)

Visiting Summer Faculty- Indiana University at Bloomington. Developed and taught a graduate course on Higgs phenomenology. (Summer 1989)

Assistant Instructor- University of Texas at Austin. Taught a physical science course for education majors. (1986-87)

Research Assistant- University of Texas at Austin. (1987-88)

Research Assistant- Fermi National Labs. Astrophysics group. (Summers of 1984,85)

Teaching Assistant- Georgia Institute of Technology. Taught a variety of undergraduate laboratory courses. (1981-83)

Instructor- U.S. Army Signal School at Fort Gordon, Georgia. (19781981)

HONORS Ford Foundation Postdoctoral Fellow, SLAC (1990-1991)

AND

President's Postdoctoral Fellow, University of California (1988-1990)

AWARDS University of Texas Graduate Fellow, 1985-1987

Fermi National Labs Graduate Fellow, 1983-1985 


\section{RECENT PUBLICATIONS (Since 1991):}

1. LHC Detection of Neutral MSSM Higgs Bosons via $g g \rightarrow h b \bar{b} \rightarrow b \bar{b} b \bar{b}$, J. Dai, J.F. Gunion, R. Vega, SMU preprint HEP-94-4, Mar 1994. Submitted for publication in Phys. Rev. D.

2. Standard Model Decays of Tau into Three Charged Leptons, D.A. Dicus, R. Vega, SMU preprint HEP-94-2, Feb. 1994. To appear in Phys. Lett. B.

3. Constraints on CP Violation in the Higgs Sector From the $\rho$ Parameter, A. Pomarol, R. Vega, Nucl. Phys. B413 3 (1994).

4. Using $b$ Tagging to Detect $t \bar{t}$-Higgs-Boson Production with $H \rightarrow b \bar{b}$, J. Dai, J.F. Gunion, R. Vega, Phys. Rev. Lett. 712699 (1993).

5. Guaranteed Detection of a MSSM Higgs Boson at Hadron Supercolliders, J. Dai, J.F. Gunion, R. Vega, Phys. Lett. B315 355 (1993).

6. The Axial Vector Coupling and Magnetic Moment of the Quark, D.A. Dicus, D. Minic, U. van Kolck, R. Vega, Phys. Lett. B284 384 (1992).

7. Isolating Purely Leptonic Signals for Strong W Scattering Using Antitagging Jet Tagging, and Lepton Isolation, D.A. Dicus, J.F. Gunion, L.H. Orr, R. Vega, Nucl. Phys. B377 31 (1992).

8. Isolating the Scattering of Longitudinal $W^{+}$'s at the SSC Using Like Sign Dileptons, D.A. Dicus, J.F. Gunion, R. Vega, Phys. Lett. B258 475 (1991).

9. Naturalness Problems for $\rho=1$ and Other Large One Loop Effects for a Standard Model Higgs Sector Containing Triplet Fields, J.F. Gunion, R. Vega, J. Wudka, Phys. Rev. D43 2322 (1991). 


\section{Curriculum Vitae}

Benjamin Grinstein

September 8, 1994

Born:

Citizenship:

August 23, 1958; Mexico City, Mexico.

Marital Status:

Mexican. (Permanent Resident, USA).

Married, two children.

Mailing Address:

University of California, San Diego

Departmentof Physics -0319

Phone(work):

La Jolla, California 92093-0319

Fax:

Phone(home):

(214)768-4075 (619)534-7142

(214)768-4095 (619)534-0173

(214) $931-8930$

Email:

ben@mail.physics.smu.edu

Education

- Universidad Iberoamericana (Mexico), 1976-1978 Biomedical Eng. program (Did not graduate)

- Centro de Investigación y Estudios Avanzados del IPN (Mexico), 19781980

M.S. in Physics: June 23, 1980

- Harvard University, 1980-1984

Ph.D. in Physics: June 6, 1984

Jobs

- ELPROSA (Mexico City): Electronic Design, May, 1977 - Dec, 1977

- IPESA (Mexico City): Computer Programming, Dec, 1977 - Jun, 1978

- U.Iberoamericana (Mexico City): Physics Teacher, Jan, 1980 - May, 1980

- Harvard University: Teaching Fellow (Physics), Jun, 1981 - Jul, 1984

- California Institute of Technology: Junior Research Associate, Sep, 1984 - Aug, 1987

- Lawrence Berkeley Laboratories: Postdoctoral Fellow, Sep, 1987 - Aug, 1988 
- Fermi National Accelerator Laboratory: Associate Scientist, Sep, 1988 Aug, 1989

- Harvard University: Assistant Professor, Sep, 1989 - May, 1991

- Harvard University: Associate Professor, Jun, 1991 - Dec, 1992

- Superconducting Super Collider: Senior Scientist, Sep, 1991 - Jan, 1994

- Souther Methodist University: Visiting Adjunct Associate Professor, Feb, 1994 -Aug. 1994.

- University of California, San Diego: Professor, July 1994- present

Honors and Prizes

- Whiting Fellow, Harvard, 1981

- Merit Fellow, Harvard, 1983

- Tolman Fellow, California Institute of Technology, 1984

- Milton Fund Award, Harvard, 1989

- Sloan Foundation Fellow, 1990

- Tozier Fund Award, Harvard, 1991

- Clark Fund Award, Harvard, 1991

Conference and Symposia Organization, and Other Service Functions

- Convenor, XIII Warsaw Symposium on Elementary Particle Physics, Kazimierz, Poland, May, 1990

- Convenor, XXVI Inetrnational Conference on High Energy Physics, Dallas, USA, August, 1992

- Convenor, 7th Meeting of the American Physical Society, Division of Particles and Fields, Batavia, USA, November, 1992

- Convenor, Workshop on B Physics at Hadron Accelerators, Snowmass, USA, June, 1993

- Local Organizing Committee, The XI International Symposium on Lattice Field Theory, Dallas, USA, October, 1993 
- Scientific Advisory Committee, Aspen Winter Physics Conference on Elementary Particle Physics, Aspen, USA, January, 1994

- Local Organizing Committee, 1994 Meeting of the Division of Particles and Fields of the American Physical Society, Albuquerque, USA, to be held August, 1994

- National Science Foundation's NYI Fellowship Panel, April 1994 


\section{Stephan T. Riemersma}

Curriculum Vitae

Southern Methodist University

Dallas, Texas 75275

steve@caitlin.physics.smu.edu
Work Phone: (214) 768-1263

Work Fax: (214) 768-4095

Home Phone: (214) 750-0791

\section{Education:}

- Ph.D. in Theoretical Particle Physics, State University of New York at Stony Brook, May, 1993.

Dissertation Title: Deep-Inelastic Heavy-Flavor Electroproduction.

Advisor: Prof. J. Smith

- M.A. in Physics. State University of New York at Stony Brook, May 1990.

- B.S. in Physics. Georgia Institute of Technology. June 1988. With High Honors.

\section{Academic Positions:}

- Southern Methodist University, Department of Physics, Theoretical Particle, Physics Group. Postdoctoral Research Associate July 1993 to Present.

\section{Invited Talks:}

- International Workshop on Physics at HERA with Internal Targets, September 1993, Hamburg, Germany.

\section{Conferences:}

- DPF '94, August 1994, Albuquerque, New Mexico.

- Radiative Corrections, June-July 1994, Gatlinburg, Tennessee.

- International Workshop on Physics at HERA with Internal Targets, September 1993, Hamburg, Germany.

- DESY Theory Workshop on Quantum Chromodynamics, September-October 1993, Hamburg, Germany.

- Workshop on B Physics at Hadron Accelerators, June-July 1993, Snowmass, Colorado. 


\section{Recent Refereed Publications}

1) Difficulties in Determining the Gluon Distribution from Electroproduction of Charmed Quarks,

S. Riemersma, J. Smith, and W.L. van Neerven, Phys. Lett. B282 (1992), p. 171.

2) Complete $O\left(\alpha_{s}\right)$ Corrections to Heavy-Flavour Structure Functions In Electroproduction,

E. Laenen, S. Riemersma, J. Smith, W.L. van Neerven, Nucl. Phys. B392 (1993), p. 162.

3) On the Heavy-Quark Content of the Nucleon,

E. Laenen, S. Riemersma, J. Smith, W.L. van Neerven,

Phys. Lett. B291 (1992), p. 325.

4) $O\left(\alpha_{s}\right)$ Corrections to Heavy-Flavour Inclusive Distributions in Electroproduction, E. Laenen, S. Riemersma, J. Smith, W.L. van Neerven, Nucl. Phys. B392 (1993), p. 229.

5) Deep-Inelastic Heavy-Flavor Electroproduction,

S. Riemersma,

Ph.D. Dissertation.

6) Complete Next to Leading Order QCD Corrections to the Photon Structure Functions $F_{2}^{\gamma}\left(x, Q^{2}\right)$ and $F_{L}^{\gamma}\left(x, Q^{2}\right)$,

E. Laenen, S. Riemersma, J. Smith, and W.L. van Neerven,

Phys. Rev. D49 p. 5753.

7) Leptoproduction of Heavy Quarks in the Fixed and Variable Flavor Schemes, F. Olness and S. Riemersma, SMU HEP 94-21, Submitted to Physical Review.

8) Fits to the $O\left(\alpha_{s}\right)$ Corrected Coefficient Functions of Heavy-Flavor Structure Functions, S. Riemersma, J. Smith, and W.L. van Neerven, in preparation.

\section{Other Publications}

9) Charm Production at HERA,

S. Riemersma, Proceedings of DPF '92 The Fermilab Meeting, p. 984.

10) B-Quark Production at Hadron Colliders,

S. Riemersma and R. Meng,

SMU HEP 93-08, ANL-HEP-CP-93-66,

Proceedings of Workshop on B-Physics at Hadron Accelerators, Snowmass '93.

11) Leptoproduction of Heavy Quarks in the Fixed and Variable Flavor Schemes, F. Olness and S. Riemersma Proceedings of DPF '94. 


\section{RESUME}

Name:

Field of Interest:
Doris C. Rosenbaum High Energy Physics Theoretical

Degrees:

B.S. Wellesley College (1957)

Ph.D. Northeastern University (1969)

Professional Experience:

1959 University of Maryland, Physics Department

Graduate Teaching Assistant

1967 - 69 Northeastern University, Physics Department Graduate Teaching Assistant

1968 Los Alamos Scientific Laboratory

Visiting Scientist (Summer)

$1974 \quad$ Virginia Polytechnic Institute and State University

Physics Department Lecturer

1975 - 78 Virginia Polytechnic Institute and State University Physics Department Adjunct Assistant Professor

1974 - 76 Northeastern University, Physics Department Visiting Scientist (Summer)

1975 - 78 Editor, University Publications, Inc., Blacksburg, Virginia

1978 - 90 Visitor, University of Maryland

1990 Consultant, TIME -LIFE Books

(Volume on "Frontiers of Time")

1990 - Southern Methodist University Scholar in Residence

1992 - 93 Superconducting Super Collider Guest Scientist 
Name: $\quad$ Doris C. Rosenbaum

Publications:

$\underline{\text { Book }}$

"Paths to Research in Electricity and Magnetism," D. C. Teplitz, Editor, Plenum Publishing Corporation, New York, 1982 (Plenum Press, NY).

Open Literature:

"Numerical Solution of the Pion - Pion Strip Approximation N/D Equation," D. C. Teplitz and V. L. Teplitz, Phys. Rev. 137, B142 (1965).

"A Fortran IV Program for Solving N/D Strip - Approximation Equations," D. C. Teplitz and V. L. Teplitz, University of California Radiation Laboratory Report No. 11696 (1964) (Unpublished).

"Theory of the D13 Pion - Nucleon Amplitude," R. Aaron, R. Amado, D. C. Teplitz, and J. Young, Phys. Rev. 187, 2047 (1969).

"Three Body Intermediate States in Pion - Nucleon Scattering," D. C. Teplitz, Thesis, Northeastern University, (1969).

"Unitarity Effects in Pion Production Isobar amplitudes," R. Aaron, R. H. Thompson, R. D. Amado, R. A. Arndt, D. C. Teplitz, and V. L. Teplitz, Phys. Rev. D12. 1984 (1975).

"Isobar Formalism and one Pion Exchange Partial - Wave Cross Sections in Nucleon-Pion into Nuclẹon Two-Pions," R. Aaron, R. D. Amado, R. A. Arndt, Y. N. Goradia, D. C. Teplitz, and V. L. Teplitz, Phys. Rev. D16, 50 (1977).

"Observational Limits on the Magnetic Monopole Structure of the Proton" J. J. Broderick, J. R. Ficenec, D. C. Teplitz, and V. L. Teplitz, Phys. Rev. 19, 1046 (1979).

"Effects of Proton Decay on the Cosmological Future." D. A. Dicus, J. R. Letaw, D. C. Teplitz, and V. L. Teplitz, Astrophysical Journal 252, 1 (1982).

"The Future of the Universe" with D. A. Dicus, V. L. Teplitz, and J. R. Letaw, Scientific American March, 1983. Reprinted in "Physics in the Cosmos," (1989).

"Bounds on Dark Matter in Solar Orbit," with J. D. Anderson, et al, Astrophysical Journal $\underline{342}$, 539, (1989).

"Improved Bounds on Non-Luminous Matter in Solar Orbit" with Anderson et al, D. A. Dicus and V. L. Teplitz, submitted to Astrophysical Journal.

Other :

"What is the Higgs Boson and Why Do We Want To Find It?" with I. Butterworth, M. Butterworth and D.C. Rosenbaum, one page essay solicited by U.K. Science Minister (one of 5 winning entries out of 120 submitted), 1993. 
I. Education

S. B. Massachusetts Institute of Technology (1958)

Ph.D. University of Maryland (1962)

II. Experience in Higher Education

1962-64 Lawrence Radiation Laboratory, Berkeley, California

1964-65 C.E.R.N., Geneva, Switzerland, NATO Fellow

1966-70 Massachusetts Institute of Technology

1970-73 Massachusetts Institute of Technology

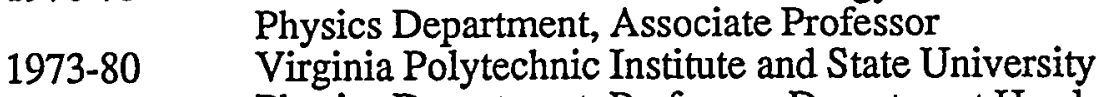

$1990-$

Professor and Chair

II. Experience other than Higher Education

A. Physics

1965

1967

1974

1975

1976

1980

$1987-88$

1990-93
MATSCIENCE, Madras, India, invited Lecturer (Summer) Brandeis Summer Institute on Theoretical Physics, invited Lecturer Massachusetts Institute of Technology, Visiting Scientist (Summer) Brookhaven National Laboratory, Visiting Scientist (Summer) Massachusetts Institute of Technology, Visiting Scientist (Summer)

Brookhaven National Laboratory, Visiting Scientist (Summer)

National Science Foundation

Program Director for Theoretical Physics

B. $\underline{\text { Other }}$

1978-90 U.S. Arms Control and Disarmament Agency, Strategic Affairs Division, Physical Science Officer

1979 Advisor in the U.S. Delegations to the second and third sessions of U.S.-U.S.S.R. negotiations on anti-satellite weapons

1981-90 ACDA, Deputy to Chief, Strategic Affairs Division

1983-86 Chair, Interagency Working Group on Antisatellite Arms Control/Defense and Space Issues

$1990 \quad$ Advisor, Round XIII, Strategic Arms Reduction Talks

1989-90 Chair, Interagency Backstopping Committee for

Strategic Arms Reduction Talks

1990- Consultant, ACDA

1992 Consultant, Meridian Corporation

1991-94 Superconducting Super Collider

Senior Advisor on International Coordination

Fellow, American Physical Society 
IV. Publications

A. Papers published in refereed journals:

[1983 and after. Full list of 65 publications available.]

1. "Evasion of the Parker Bound on Monopoles," with D. A. Dicus, Nature 303, 408 (1983).

2. "Upper Bound on the Decay Constant of Familons," with D. A. Dicus, Phys. Rev. D28, 1778 (1983).

3. "An Isobar Model Partial Wave Analysis $\pi N \rightarrow \pi \pi N$ in the c.m. Energy Range 1320-1930 MeV," with D. M. Manley, R. A. Arndt, and Y. Goradia, Phys. Rev. D30, 904 (1984).

4. "Finite Temperature Corrections to the van der Waals Potential," L. Chiu, D. Dicus, E. Kolb, B. Joseph, and M. Turner, Phys. Rev. A31, 1458 (1985).

5. "Unstable Particles and the Large-and Small-scale Dark Matter Problems," with D. A. Dicus, Phys. Rev. D34, 934 (1986).

6. "Bounds on Dark Matter in Solar Orbit," with J. D. Anderson, E. L. Lau, A. H. Taylor, D. A. Dicus, and D. C. Teplitz, the Astrophysical Journal $\underline{342}, 539$ (1989).

7. "Implications of Relativistic Gas Dynamics for Neutrino-Neutrino Cross Sections," with P. B. Pal, S. Nussinov, and D. A. Dicus, Phys. Letter 218, 84 (1989).

8. "Coupled Channel Vector-Boson Scattering in the N/D Method" with D. A. Dicus, to be published in Physics Review.

9. "Improved Bounds on Non-Luminous Matter in Solar Orbit" with Anderson et al, D. A. Dicus and D. Rosenbaum, to be submitted to Astrophysical Journal.

B. Books or Contributions to books, Symposia and Popular Journals (partial)

1. Homology and Feynman Integrals. (With Rudolph C. Hwa). Mathematical Physics Monograph Series, W. A. Benjamin, New York, 1966.

2. "Magnetic Monopoles," with J. R. Ficenec in Electromagnetism: Paths to Research, Plenum publishing Co., 1982 (D. C. Teplitz, Editor).

3. "The Future of the Universe," with D. A. Dicus, J. R. Letaw, and D. C. Teplitz, Scientific American, March 1983, reprinted in Particle Physics in the Cosmos, (R. A. Carrigan, Jr. and W. P. Trower, ed., W. H. Freeman and company, New York, 1989.

4. "Is there a Delta-Delta-Pi Problem," with D. A. Dicus, in G. F. Chew Symposium, (C. Taar, ed.) 1986. 